\title{
Atlas of Tidal Elevation and Current Observations on the Northeast
}

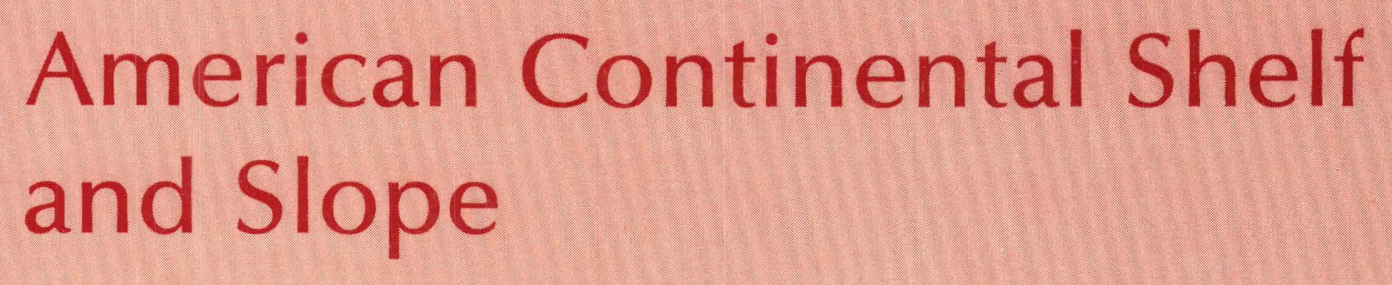

\section{U.S. GEOLOGICAL SURVEY BULLETIN 1611}





\section{Atlas of Tidal Elevation and Current Observations on the Northeast American Continental Shelf and Slope}

By John A. Moody, Bradford Butman, Robert C. Beardsley, Wendell S. Brown, Peter Daifuku, James D. Irish, Dennis A. Mayer, Harold O. Mofjeld, Brian Petrie, Steven Ramp, Peter Smith, and W. R. Wright 


\title{
DEPARTMENT OF THE INTERIOR WILLIAM P. CLARK, Secretary
}

\author{
U.S. GEOLOGICAL SURVEY \\ Dallas L. Peck, Director
}

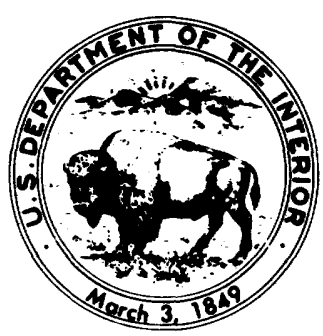

UNITED STATES GOVERNMENT PRINTING OFFICE: 1984

For sale by the Distribution Branch, U.S. Geological Survey, 604 South Pickett Street, Alexandria, VA 22304 


\title{
CONTENTS
}

\author{
Abstract 1 \\ Introduction 1 \\ Acknowledgments 3 \\ Data set 3 \\ Data analysis 3 \\ Results and discussion 6 \\ Tidal elevation 6 \\ Tidal character 6 \\ $\mathbf{M}_{2}$ tide 7 \\ $\mathrm{N}_{2}$ tide 8 \\ $\mathrm{S}_{2}$ tide 8 \\ $K_{1}$ tide 8 \\ $\mathrm{O}_{1}$ tide 8 \\ Tidal current 8 \\ $M_{2}$ tidal current 8 \\ $\mathrm{N}_{2}$ tidal current 11 \\ $\mathrm{S}_{2}$ tidal current 11 \\ $\mathrm{K}_{1}$ tidal current 11 \\ $O_{1}$ tidal current 11 \\ Summary 12 \\ References cited 12 \\ Appendixes: I-Instrument description 97 \\ II- $\mathbf{M}_{2}$ bottom current estimates 98 \\ III-Tide generation 100
}

FIGURES

1. Phase relationships 5

2. Vertical structure of normalized $M_{2}$ near-bottom current 7

3. Longshelf variation in UMAJOR for the $M_{2}$ and $K_{1}$ constituents 9

\section{PLATES}

1. Location of stations (in pocket)

2. Vertical location of current observations (in pocket)

3. $F$ values for study region 105

4. Coamplitude and cophase lines for the $M_{2}$ (12.42 hours) semidiurnal tide 106

5. Coamplitude and cophase lines for the $N_{2}$ (12.66 hours) semidiurnal tide 107

6. Coamplitude and cophase lines for the $S_{2}(12.00$ hours) semidiurnal tide 108

7. Coamplitude and cophase lines for the $K_{1}(23.93$ hours) diurnal tide 109

8. Coamplitude and cophase lines for the $O_{1}(25.82$ hours) diurnal tide 110

9. Magnitude and phase of the $M_{2}$ surface current 111

10. Representative $\mathbf{M}_{2}$ tidal ellipses 112

11. $M_{2}$ currents at 1 meter above bottom 113

12. Phase difference (elevation minus current) for the $M_{2}$ tide 114 
13. Time sequence of $\mathbf{M}_{2}$ sea-surface elevation contours and current vectors on the Continental Shelf (in pocket)

14. Magnitude and phase of the $N_{2}$ surface current 115

15. Magnitude and phase of the $S_{2}$ surface current 116

16. Spring-neap variation of amplitude of the semidiurnal tidal current caused by the $S_{2}$ solar tide expressed as a percent of the $M_{2}$ tidal current 117

17. Magnitude and phase of the $K_{1}$ surface current 118

18. Representative $K_{1}$ ellipses 119

19. Phase difference (elevation minus current) for the $K_{1}$ tide 120

20. Magnitude and phase of the $O_{1}$ surface current 121

21. Representative $O_{1}$ tidal ellipses

122

\section{TABLES}

1. Tidal constituents 3

2. Estimates of the error in tidal elevation and current $\mathbf{5}$

3. Data references 17

4. Tidal constants for elevation 24

5. $\mathbf{M}_{2}$ tidal current parameters 33

6. $\mathrm{N}_{2}$ tidal current parameters 47

7. $\mathrm{S}_{2}$ tidal current parameters $\mathbf{5 8}$

8. $\mathrm{K}_{1}$ tidal current parameters $\mathbf{7 0}$

9. $\mathrm{O}_{1}$ tidal current parameters $\mathbf{8 2}$ 


\title{
Atlas of Tidal Elevation and Current Observations on the Northeast American Continental Shelf and Slope
}

\author{
By John A. Moody ${ }^{1}$, Bradford Butman ${ }^{1}$, Robert C. Beardsley ${ }^{2}$, Wendell S. Brown ${ }^{3}$, \\ Peter Daifuku ${ }^{4}$, James D. Irish ${ }^{3}$, Dennis A. Mayer ${ }^{5}$, Harold O. Mofjeld $^{6}$, \\ Brian Petrie ${ }^{7}$, Steven Ramp ${ }^{8}$, Peter Smith ${ }^{7}$, and W. R. Wright ${ }^{8}$
}

\begin{abstract}
Measurements of sea-surface elevation or bottom pressure at 100 stations and measurements of current at various depths at 139 stations on the North American Continental Shelf from Cape Hatteras to the Laurentian Channel have been analyzed using either the harmonic or the response method for five tidal constituents: the semidiurnal tides $M_{2}$ (12.42 hours), $\mathrm{N}_{2}$ (12.66 hours), and $\mathrm{S}_{2}$ (12.00 hours), and the diurnal tides $\mathrm{K}_{1}$ (23.93 hours) and $\mathrm{O}_{1}$ (25.82 hours). The amplitude and phase of elevation and of east and north current for the five constituents are presented in tables. The ellipse representation of the current is also tabulated. In addition, plates are presented showing tidal elevation and surface current (for $\mathrm{M}_{2}, \mathrm{~N}_{2}, \mathrm{~S}_{2} \mathrm{~K}_{1}$ and $\mathrm{O}_{1}$ ) and representative current ellipses (for $M_{2}, K_{1}$ and $O_{1}$ ). This is the first compilation of tidal elevation over this region of the Continental Shelf for constituents other than $\mathrm{M}_{2}$ and of tidal currents at depths other than at the surface.

The coamplitude and cophase lines for all semidiurnal tides are similar and indicate a co-oscillating tide in the Middle Atlantic Bight and on the Scotian Shelf, and a nearresonant tide in the Gulf of Maine. There is an amplitude minimum of the sea-surface elevation over Nantucket Shoals
\end{abstract}

'U.S. Geological Survey, Woods Hole, MA 02543

${ }^{2}$ Woods Hole Oceanographic Institution, Woods Hole, MA 02543

${ }^{3}$ Department of Earth Science, University of New Hampshire, Durham, NH 03824

${ }^{4}$ EG\&G Environmental Consultants, Waltham, MA 02154

${ }^{5}$ Atlantic Oceanographic and Meteorological Laboratories, National Oceanic and Atmospheric Administration, Miami, FL 33149

'Pacific Marine Environmental Laboratories, National Oceanic Atmospheric Administration, Seattle, WA 98105

'Bedford Institute of Oceanography, Dartmouth, Nova Scotia B2Y 4A2

${ }^{8}$ Northeast Fisheries Center, National Marine Fisheries Service, Woods Hole, MA 02543 and Georges Bank. The diurnal tides $\left(\mathrm{K}_{1}\right.$ and $\left.\mathrm{O}_{1}\right)$ are dominated by an amphidromic point near Sable Island and a secondary amphidromic point in the Middle Atlantic Bight. Along the remainder of the shelf the diurnal tide seems to be a combination of Kelvin and shelf waves.

The major axis of the $M_{2}$ tidal current ellipses are generally oriented perpendicular to the local isobaths except near the coast and in the Great South Channel and Northeast Channel. In contrast, the major axis of the ellipses of the two diurnal constituents are generally oriented parallel to the isobaths. The amplitude of the $M_{2}, K_{1}$, and $O_{1}$ surface current is weakest at the outer edge of the shelf, reaches a maximum midway across the shelf, and then decreases toward the coast. The variability of the tidal current estimates is largest near the shelf break where some contribution from a baroclinic tide is expected.

Observations at five stations, where current measurements were made at 1 and at 10 to 20 meters above the bottom, were used to empirically determine the vertical structure of the $M_{2}$ semidiurnal current near the bottom. The amplitude of the $M_{2}$ bottom current is typically about 50 percent of the amplitude of the near-surface current. The empirical curve was used to estimate the bottom tidal currents ( 1 meter above the bottom) at 78 locations on the Continental Shelf where measurements were available only at 10 - to $20-\mathrm{m}$ above the bottom.

\section{INTRODUCTION}

This atlas is a compilation of tidal constants for current and pressure made at $\mathbf{2 1 6}$ locations on the North American Continental Shelf from Cape Hatteras to the Laurentian Channel. In recent years, measurements of current and bottom pressure have been made over the Continental Shelf and Slope in numerous field experiments conducted by many investigators. The primary objective of most of these field programs was to study the 
kinematics and dynamics of the low-frequency and seasonal mean circulation. While some of the experiments were designed specifically to examine the tides, the spatial coverage of most of the individual experiments was generally limited. As a result, tidal analysis of the new current and pressure data obtained in these experiments has been scattered and incomplete and has generally been limited to the dominant $\mathbf{M}_{2}$ semidiurnal component. This atlas presents in graphical and tabular form the tidal constants determined from data obtained in these recent field experiments. In addition, constants obtained from the literature for selected coastal stations are also included for completeness. A brief description of the major features of the observed surface tides and tidal currents is also presented.

Previous observations of the tides in the region are limited. Haight (1942) reported surface tidal-current measurements made at anchored lightships landward of the $60-\mathrm{m}$ isobath. A few tidal constants from pressure observations made along the edge of the shelf in water more than $100 \mathrm{~m}$ deep were compiled by Cartwright and others (1979). There are several references to tidal constants, most as parts of papers on other topics (Greenberg, 1975; Beardsley and others, 1977b; Mayer and others, 1979; Vermersch and others, 1979; Moody and Butman, 1980; Mayer, 1982a and 1982b). Tidal constants along the United States coast are available from the Tides Branch of the National Ocean Service and along the Canadian coast from the Canadian Hydrographic Service (1966 and 1969a). Syntheses of semidiurnal tidal observations have been presented by Redfield (1953) for the Gulf of Maine and by Redfield (1958) and Swanson (1976) for the Middle Atlantic Bight. A synthesis of $K_{1}$ tidal observations has been presented by Daifuku (1981).

The data presented in this atlas were obtained along the North American Continental Shelf from Cape Hatteras to the Laurentian Channel (plate 1). This region can be divided geographically into four major areas: the Middle Atlantic Bight, Georges Bank, the Gulf of Maine, and the Scotian Shelf. The Middle Atlantic Bight extends from Cape Hatteras northward to Nantucket Shoals. The shelf is quite narrow $(<75 \mathrm{~km})$ and shallow $(\sim 40 \mathrm{~m})$ in the southern part of the bight and becomes wider $(125 \mathrm{~km})$ and deeper $(\sim 60 \mathrm{~m})$ south of Cape Cod. The sea floor in the Middle Atlantic Bight is quite smooth. To the northeast of the Middle Atlantic Bight is Georges Bank, a shallow submarine bank located on the south side of the Gulf of Maine. The bank is about $300 \mathrm{~km}$ long and $150 \mathrm{~km}$ wide and is separated from Nantucket Shoals by the Great South Channel ( $\sim 70 \mathrm{~m}$ deep) and from the Scotian Shelf by Northeast Channel ( $\sim 230 \mathrm{~m}$ deep). The water on the crest of Georges Bank is generally $30-40 \mathrm{~m}$ deep, although less than $5 \mathrm{~m}$ deep in some places. The Gulf of Maine is about $400 \mathrm{~km}$ long and $250 \mathrm{~km}$ wide.
Three major basins and numerous smaller basins in the Gulf of Maine are more than $260 \mathrm{~m}$ deep. The basins are separated by a series of ledges, ridges, and swells. The bottom topography is complex and rough, especially in the north-central region and west of Nova Scotia. The Scotian Shelf is wider $(150-200 \mathrm{~km})$, deeper, and topographically rougher than the Middle Atlantic Bight. Browns Bank on the western end of the shelf, the shelf around Sable Island, and Banquereau Bank adjacent to the Laurentian Channel all are less than $60 \mathrm{~m}$ deep. There are two deep basins $(>240 \mathrm{~m})$ and two banks $(<60 \mathrm{~m})$ southwest of Halifax. The Laurentian Channel, which is greater than $400 \mathrm{~m}$ deep, cuts across the Scotian Shelf between Nova Scotia and Newfoundland and is the northern limit of this study.

The tides and tidal currents on the Continental Shelf clearly affect many physical processes, and thus a description of the tides has many applications. For example, the strength of the tidal currents influences the surface-sediment distribution and the short- and longterm fate of material and pollutants introduced into the water column (Bothner and others, 1981; Twichell and others, 1981). The tides also influence the extent of vertical mixing on Georges and Browns Bank and on Nantucket Shoals, and thus partially determine the location of hydrographic fronts (Garrett and others, 1978). The clockwise mean circulation observed around Georges and Browns Bank may be partially caused by rectification of the strong tidal currents (Loder, 1980; Butman and others, 1982; Hopkins and Garfield, 1982; Butman and others, 1983; Smith, 1983). Both the vertical mixing and the clockwise circulation may indirectly affect the biological productivity of the region by controlling the vertical distribution of nutrients and organisms and the advection of eggs and larvae.

A description of the tidal currents is important for parameterization of bottom stress in analytical and numerical models of the subtidal circulation where the tidal currents are not explicitly included (Noble and others, 1983). In addition to direct effects of the tides, a description of the tides is important for removing the large but predictable tidal signal where it masks other signals of interest. For example, in order to determine the low-frequency variability of sea level from observations made by satellite, the tidal signal must be known. Finally, tidal information has been used to improve geophysical models of tidal loading of the earth's crust (Beaumont and Boutilier, 1978).

This atlas of tidal constants for elevation and current includes recent tidal observations as well as selected observations from the literature. It is intended to serve as a reference document of the tides along the northeast coast of North America. It is the first compilation to include the $\mathrm{M}_{2}, \mathrm{~N}_{2}, \mathrm{~S}_{2}, \mathrm{~K}_{1}$, and $\mathrm{O}_{1}$ constituents both for sea-level elevation and for currents at various depths. 


\section{ACKNOWLEDGMENTS}

W. Boicourt (Chesapeake Bay Institute, Baltimore, Md.), D. Goodrich (SUNY, Stony Brook, N.Y.), and A. Petrillo (URI, Kingston, R.I.) provided the tidal constants for data at stations MAB, EPAl and CXL, respectively. Support for J. Moody and B. Butman was provided by the U.S. Geological Survey and by the Bureau of Land Management under Memoranda of Understanding AA551-MU9-4 and AA551-MU0-18, and Interagency Agreements AA551-IA1-17 and IA851-IA2-26. Support for B. Beardsley and P. Daifuku was provided by the Ocean Science Division of the National Science Foundation. H. Mofjeld was supported by NOAA through the MESA N.Y. Bight Project. S. Ramp and W. Wright were supported by the National Marine Fisheries through the Marine Resources Monitoring Assessment and Prediction Program (MARMAP).

\section{DATA SET}

The tidal constants (amplitude and phase) for elevation and current in this atlas have been compiled from observations made by several investigators at 216 stations on the Continental Shelf and Slope from Cape Hatteras northward to the Laurentian Channel (plate 1). For convenience, the stations are grouped geographically into twelve areas: Scotian Shelf, Gulf of Maine, Bay of Fundy, Northeast Channel, Georges Bank, Great South Channel, Lydonia Canyon, Nantucket Shoals, New England Shelf, Middle Atlantic Bight, southern MidAtlantic Bight, and Oceanic (see plate 1 and tables 4-9). Table 3 lists the stations alphabetically, the type of instrument used to make the measurement, the method of tidal analysis (see "Data analysis" below), and the data source. The station names used by the investigators who made the measurements have been used to simplify comparison with references in other papers.

The vertical distribution of the current observations made in each region is shown in plate 2 . There were typically 1 to 4 observations at each station. Observations $1 \mathrm{~m}$ above bottom (hereafter mab) were obtained at a few stations. The spatial coverage is densest in the northern Middle Atlantic Bight and on Georges Bank and sparser in the southern Middle Atlantic Bight and on the Scotian Shelf. The measurements in the Gulf of Maine are extremely limited. Only a few measurements were made in water depths greater than $100 \mathrm{~m}$, primarily on the Scotian Slope, around Lydonia Canyon, and on the slope between longitudes $70^{\circ}$ and $73^{\circ} \mathrm{W}$.

The data presented in this atlas were obtained in several ways. All data were compiled, tabulated, and plotted by J. Moody. In most cases, investigators computed the tidal constants from their data using their own puted the tidal constants from their data using their own tidal analysis programs. In other cases, investigators provided the original time series, and the tidal constants were computed by J. Moody. Additional tidal constants, primarily of coastal sea level, were obtained from the literature. All investigators who contributed raw data or tidal constants from more than one station are authors of this atlas. J. Moody and B. Butman wrote the text.

\section{DATA ANALYSIS}

The tidal constants (amplitude and phase) for elevation and east and north current components were determined for the five major constituents listed in table 1. These five tidal constituents typically account for more than 80 percent of the total variance of current observations in the Georges Bank region (based on observations at stations A, C, D, and L), and for 30 to 40 percent in the Middle Atlantic Bight (based on observations at station MB). On the Continental Slope, the five constituents accounted for only 5 percent of the total current variance (based on observations at station MF). The five major tidal constituents (based on observations at stations A, B, $\mathrm{D}, \mathrm{K}, \mathrm{MB}, \mathrm{MC}, \mathrm{MD}, \mathrm{ME}, \mathrm{P}, \mathrm{Q}$, and $\mathrm{R}$ ) accounted for more than 85 percent of the total variance in sea-level elevation and more than 93 percent of the variance at periods shorter than 33 hours.

Tidal constants were determined by using either the response method (Munk and Cartwright, 1966) or the harmonic method (Schureman, 1941; Dennis and Long, 1971). At two stations (M and LT5), the constants were computed by both methods, and the difference between current amplitude was less than $1 \mathrm{~cm} / \mathrm{s}$ for all constituents except the $S_{2}$ semidiurnal tide. The phase estimates differed by $4^{\circ}$ to $6^{\circ}$ and the estimates of ellipse orientation differed by $4^{\circ}$ to $30^{\circ}$ with the larger difference occurring again for the solar semidiurnal constituent.

Bottom-pressure observations on the shelf and slope, which were converted to height in centimeters for comparison with elevations measured at coastal stations, are referred to as tidal elevations $(1 \mathrm{mb}=0.995 \mathrm{~cm})$. All

Table 1. Characteristics of the five tidal constituents described in this atlas. Equilibrium tide is computed for latitude $42^{\circ} \mathrm{N}$.

\begin{tabular}{|c|c|c|c|c|}
\hline Symbol & $\begin{array}{c}\text { Period } \\
\text { (hr) }\end{array}$ & $\begin{array}{l}\text { Speed } \\
\text { (deg/hr) }\end{array}$ & $\begin{array}{l}\text { uilibrium } \\
\text { Tide } \\
(\mathrm{cm})\end{array}$ & Description \\
\hline $\mathbf{M}_{2}$ & 12.4206 & 28.984 & 13.4 & Lunar semidiurnal. \\
\hline $\mathbf{N}_{2}$ & 12.6583 & 28.440 & 2.6 & Large lunar elliptic. \\
\hline $\mathrm{S}_{2}$ & 12.0000 & 30.000 & 2.9 & Solar semidiurnal. \\
\hline $\mathbf{K}_{1}$ & 23.9345 & 15.041 & 14.1 & Luni-solar diurnal. \\
\hline $\mathrm{O}_{1}$ & 25.8193 & 13.943 & 10.0 & Lunar diurnal. \\
\hline
\end{tabular}


observations of phase were converted to the Greenwich phase, G, which is independent of both longitude and the time meridian (fig. 1). National Ocean Service tidal constants are generally listed in terms of the local phase, $\kappa$, and have been converted according to

$$
\mathrm{G}=\kappa+\mathrm{pL},
$$

where $p$ equals 1 and 2 for diurnal and semidiurnal tides, respectively, and $L$ is the west longitude of the station expressed in degrees. Canadian Hydrographic Service tidal constants are listed by the local Greenwich phase, g, and have been converted according to

$$
\mathbf{G}=\mathrm{g}+\mathrm{aS} / 15,
$$

where $a$ is the angular constituent speed in degrees per hour (see table 1) and $S$ is the west longitude of the time meridian expressed in degrees.

The tidal current can be represented as eastward and northward current components or as a tidal ellipse. The tidal ellipse parameters are the amplitude of the major axis (UMAJOR), amplitude of the minor axis (UMINOR), orientation of the major axis (ORIEN), and time of maximum current (PHASE). If the east and north currents at frequency $\omega$ are expressed as

$$
\begin{aligned}
\text { east } & =U \cos \left(\omega t-\phi_{\mathrm{u}}\right), \\
\text { north } & =\mathrm{V} \cos \left(\omega t-\phi_{\mathrm{v}}\right),
\end{aligned}
$$

where $(U, V)$ and $\left(\phi_{u}, \phi_{v}\right)$ are the amplitude and phase for the east and north current components, respectively, then the corresponding ellipse parameters are given by

$$
\begin{aligned}
& \text { UMAJOR }=\left|U_{+}\right|+\left|U_{-}\right| \text {, } \\
& \text { UMINOR }=\left|U_{+}\right|-\left|U_{-}\right| \text {, } \\
& \text { PHASE }=1 / 2 \tan ^{-1}\left\lfloor\frac{2\left(A_{1} B_{1}+A_{2} B_{2}\right)}{A_{1}{ }^{2}-B_{2}{ }^{2}+A_{2}{ }^{2}-B_{1}^{2}}\right\rceil, \\
& \text { ORIEN }=1 / 2 \tan ^{-1}\left[\frac{2\left(A_{1} A_{2}+B_{1} B_{2}\right)}{A_{1}{ }^{2}+B_{1}{ }^{2}-\left(A_{2}{ }^{2}+B_{2}{ }^{2}\right)}\right],
\end{aligned}
$$

where

$$
\begin{array}{ll}
\mathrm{A}_{1}=\mathrm{U} \cos \phi_{\mathrm{u}}, & \mathrm{A}_{2}=\mathrm{V} \cos \phi_{\mathrm{v}}, \\
\mathrm{B}_{1}=\mathrm{U} \sin \phi_{\mathrm{u}}, & \mathrm{B}_{2}=\mathrm{V} \sin \phi_{\mathrm{v}},
\end{array}
$$

and

$$
\begin{aligned}
& \left|U_{+}\right|=1 / 2\left[\left(A_{1}+B_{2}\right)^{2}+\left(A_{2}-B_{1}\right)^{2}\right]^{1 / 2}, \\
& \left|U_{-}\right|=1 / 2\left[\left(A_{1}-B_{2}\right)^{2}+\left(A_{2}+B_{1}\right)^{2}\right]^{1 / 2},
\end{aligned}
$$

In this representation, the orientation is measured counterclockwise from east.

Given an ellipse with major and minor axis twice the length of UMAJOR and UMINOR, and with the major axis oriented toward the ellipse orientation, ORIEN, the tidal current flows in the direction of a vector that originates at the center of the ellipse and terminates on the perimeter. The tip of the tidal-current vector sweeps around the ellipse in one tidal period and reaches a maximum equal to UMAJOR twice in a tidal cycle, when $\omega t=$ PHASE $\left( \pm 180^{\circ}\right)$. The angle that the current vector makes with north is

$$
\alpha=90^{\circ}-\tan ^{-1}\left(\left(\mathrm{~V} \cos \left(\omega t-\phi_{\mathrm{v}}\right)\right) / \mathrm{U} \cos \left(\omega \mathrm{t}-\phi_{\mathrm{u}}\right)\right) .
$$

If UMINOR is negative, the current vector rotates clockwise. If UMINOR is positive, the current vector rotates counterclockwise. The maximum excursion that a water particle travels in a tidal cycle is $U T / \pi$, where $U$ is the current amplitude (either UMAJOR or east or north component) and $T$ is the tidal period. For the $M_{2}$ tide, the tidal excursion (in $\mathrm{km}$ ) is $0.142 \mathrm{U}$, where $U$ is in $\mathrm{cm} / \mathrm{s}$.

When the tidal constants were determined by the harmonic method (Dennis and Long, 1971), the data records were broken into 29-day pieces and the constants determined for each piece. Some investigators, however, used a modified harmonic analysis that was not restricted to 29-day data pieces. For records consisting of more than one piece, the average amplitude and phase were computed as a vector average of the amplitudes and phases determined for each piece. The scalar standard deviations of the amplitude and phase (and of the ellipse parameters) were computed as an estimate of the variability of the constants. An estimate of the uncertainty for some of the constants computed by the response method was based on the 95 percent confidence limits (Daifuku, 1981). At stations for which no estimates of the confidence limits were computed, the record length is an approximate indicator of the reliability of the constants.

The average standard deviation for tidal elevation was computed for the 25 stations where standard deviations were available (table 2). The average standard deviation of elevation amplitude for all constituents ranged from 0.5 to $0.7 \mathrm{~cm}$, or about 1 percent of the amplitude for the dominant $M_{2}$, about 4 percent for the $N_{2}$ and $S_{2}$, and about 9 percent for the $K_{1}$ and $O_{1}$. The average standard deviation of phase was $1^{\circ}-3^{\circ}$ for the semidiurnal constituents and $4^{\circ}$ for the diurnal constituents.

The tidal constants for current (both amplitude and phase) were more variable than those for elevation. The average standard deviation of current for all observations at less than $50 \mathrm{~m}$ from the surface was $1.4 \mathrm{~cm} / \mathrm{s}$ for $M_{2}$ and less than $1.0 \mathrm{~cm} / \mathrm{s}$ for the other constituents (table 2). This average standard deviation for current was 9 percent 


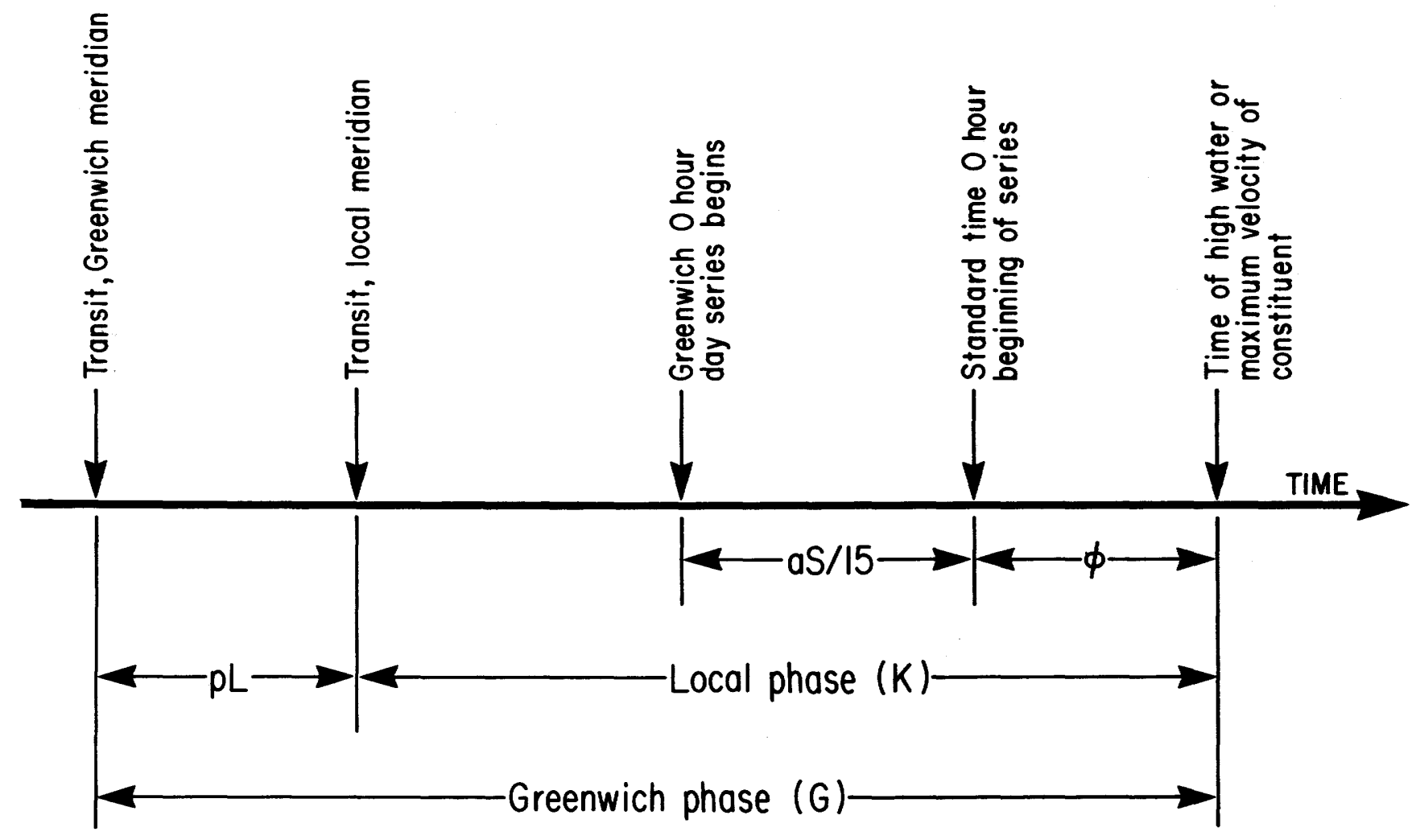

(adapted from Schureman, 1941)

Figure 1. Phase relationships. $p$, subscript of the constituent $(2=$ semidiurnal, $1=$ diurnal); $L$, longitude in degrees of the station (positive-west); S, longitude of the time meridian in degrees (positive-west); a, angular speed of the constituent; and $\phi$, phase relative to the beginning of the series (adapted from Schureman, 1941).

of the amplitude for the dominant $\mathbf{M}_{2}$ and greater than 20 percent of the amplitude for the other constituents. Standard deviation of the phase and orientation estimates were about $10^{\circ}$ for $\mathrm{M}_{2}$, and between $20^{\circ}$ and $30^{\circ}$ for $\mathrm{N}_{2}$, $\mathrm{S}_{2}, \mathrm{~K}_{1}$, and $\mathrm{O}_{1}$. In summary, these estimates suggest errors in amplitude of about $1 \mathrm{~cm}$ for elevation and about $1 \mathrm{~cm} / \mathrm{s}$ for current, and phase errors of less than $4^{\circ}$ for elevation, about $10^{\circ}$ for $\mathrm{M}_{2}$ current, and $20^{\circ}$ to $30^{\circ}$ for $\mathrm{N}_{2}, \mathrm{~S}_{2}, \mathrm{~K}_{1}$, and $\mathrm{O}_{1}$ current.

Several types of instruments were used to make the current observations reported here (see table 3). Most observations were made in water less than $100 \mathrm{~m}$ deep where oscillatory currents associated with surface waves can (by rotor pumping) increase the current speeds recorded by current meters. Speed errors can be as large as a factor of 2 for certain types of instruments on certain types of moorings (Halpern and Pillsbury, 1976; Beardsley and others, 1977). The data collected using Aanderaa instruments at shallow water stations are suspect. For example, Mayer and others (1979) found that the kinetic energy of the semidiurnal tidal currents measured using Aanderaa instruments (at 7-30 m) at station LTM in the Middle Atlantic Bight varied by a factor of 2 during the year and that the maximum values were reached in the winter months when surface waves were large. The surface measurements at LTM were decoupled from the

Table 2. Estimates of the error in tidal elevation and current for five tidal constituents. For elevation, all stations (25) with standard deviations listed in table 4 were used to compute average standard deviations of amplitude and phase. For current, all observations at depths less than $50 \mathrm{~m}$, and at 1 meter above bottom were used to compute average standard deviations of ellipse amplitude, phase and orientation.

\begin{tabular}{lccccc}
\hline & $\mathrm{M}_{2}$ & $\mathrm{~N}_{2}$ & $\mathrm{~S}_{2}$ & $\mathrm{~K}_{1}$ & $\mathrm{O}_{1}$ \\
\hline Elevation & & & & & \\
$\sigma$ Amplitude (cm) & 0.6 & 0.6 & 0.5 & 0.7 & 0.6 \\
$\sigma /$ Amplitude (\%) & 1.3 & 4.5 & 4.3 & 8.3 & 9.1 \\
$\sigma$ Phase (deg) & 1 & 3 & 2 & 4 & 4 \\
& & & & & \\
Current & 1.4 & 1.0 & 0.6 & 0.9 & 0.5 \\
$\sigma$ Amplitude (cm) & 9 & 28 & 21 & 22 & 22 \\
$\sigma /$ UMAJOR amp. (\%) & 11 & 21 & 22 & 29 & 30 \\
$\sigma$ Phase (deg) & 7 & 20 & 18 & 27 & 25 \\
$\sigma$ Orientation (deg) & & & & & \\
$\sigma$ is standard deviation & & & & & \\
\hline
\end{tabular}


subsurface float by using a special wave-following spar buoy; the errors for the instrument at $8 \mathrm{mab}$ were small because of the location of the subsurface float and the stiffness of the mooring (Mayer and others, 1979). Only surface and near-bottom measurements at LTM are included in this atlas. Observations at some other stations made by means of Aanderaa current meters (C3, C5, CMICE, LT2, LT3, MAB, NJ4, S2, and SS10) indicate a maximum in the velocity of the tidal current at mid-water depths. The tidal constants for these observations may be contaminated by rotor pumping and should be used with caution. There is no evidence to suspect observations obtained with VACM's. For example, tidal constants computed from the long-term measurements obtained at station A on Georges Bank showed no seasonality. No corrections were made to any of the tidal constants for errors caused by rotor pumping; they were tabulated as calculated by the investigators.

The tidal-current constants presented in this atlas are estimates of the amplitude and phase of the tides obtained from each data record. No attempt was made to estimate or remove the contribution of the internal tide, if any, to the measurements. In general, the presence of internal tides will probably cause increased variability in the amplitude and phase of the tidal constituents because they are transient and not usually phase-locked to the astronomical forcing. The internal tides were apparently strongest at stations on the Continental Slope where the variability was large in comparison with the variability at stations on the Continental Shelf.

Current measurements from five stations $(K, A$, $\mathrm{MB}, \mathrm{Q}$ and LCA) were selected to represent different tidal environments on the Continental Shelf. Station $\mathrm{K}$ represents a turbulent environment near the crest of Georges Bank where typical surface tidal currents are 50 to $60 \mathrm{~cm} / \mathrm{s}$ in $60 \mathrm{~m}$ of water over a mobile bottom covered by fine sand with small ripples. At station $\mathrm{A}$ on the southern flank of Georges Bank, the water depth is $85 \mathrm{~m}$; the typical tidal current is 30 to $40 \mathrm{~cm} / \mathrm{s}$, and the bottom surface sediment is fine sand with occasional small ripples. Station MB, in the Middle Atlantic Bight, is in $60 \mathrm{~m}$ of water and there are weak tidal currents of 14 to $16 \mathrm{~cm} / \mathrm{s}$ over a sand bottom (Butman and Moody, 1983). Station $\mathrm{Q}$ in $67 \mathrm{~m}$ of water has current speeds of 15 to $20 \mathrm{~cm} / \mathrm{s}$ with a smooth mud bottom. Station LCA is in $100 \mathrm{~m}$ of water at the edge of the Continental Shelf with surface tidal currents of $34 \mathrm{~cm} / \mathrm{s}$ and a bottom covered by sand. Current measurements at these five stations were made at 1 mab by means of a bottom tripod system (Butman and Folger, 1979) and at several depths above the bottom by means of an EG\&G VACM. The observations at these stations were used to determine a simple empirical method for estimating currents 1 mab at other stations. The vertical length scale for the frictional bottom layer is about $10 \mathrm{~m}$. At each station the amplitude of the major axis of the tidal ellipse (UMAJOR) was normalized by a value of UMAJOR above the bottom boundary layer, typically 20 to 30 mab. Although somewhat scattered, the normalized observations from quite different tidal environments fall on a relatively smooth curve (fig. 2). The amplitude of the major axis of tidal current ellipse 1 mab was about 0.5 times the amplitude of the major axis in the interior. The average difference in phase and ellipse orientation (interior minus bottom) was $17^{\circ}$ and $-14^{\circ}$, respectively, at stations A, K, LCA, $\mathrm{MB}$, and $\mathrm{Q}$. Thus, maximum near-bottom current occurred before maximum mid-depth current, and the nearbottom ellipse was rotated slightly to the right of the middepth ellipse.

The empirical curve (fig. 2) and the average ellipse and phase differences given above have been used to estimate the amplitude of the near-bottom tidal currents, given observations $10-30$ mab. The vertical structure of the weaker and more variable $\mathrm{N}_{2}$ and $\mathrm{S}_{2}$ components was similar to that of the $\mathbf{M}_{2}$ component. The vertical structure of the diurnal tides could not be determined because the vertical changes were much smaller than the 20 to 30 percent uncertainty of the current amplitude and $20^{\circ}$ to $30^{\circ}$ uncertainty in phase and ellipse orientation.

\section{RESULTS AND DISCUSSION}

The amplitude and the Greenwich phase for the tidal constituents $M_{2}, N_{2}, S_{2}, K_{1}$, and $O_{1}$ are listed for 100 stations in table 4 . There are 34 coastal stations, 53 stations on the Continental Shelf, 10 on the slope, and 3 oceanic reference stations. The coastal stations were selected to represent the tide along the edge of the shelf; stations in local harbors or estuaries are not included. Data from only a few of the Canadian stations along the coast of Nova Scotia and in the Bay of Fundy are tabulated in this atlas (for all stations, see Canadian Hydrographic Service, 1969a). The amplitude and Greenwich phase of the northward and eastward current components and the ellipse parameters for the five tidal constituents $M_{2}, N_{2}, S_{2}, K_{1}$, and $O_{1}$ are listed in tables 5-9.

The tidal elevation and current observations are briefly discussed in the following sections and presented graphically as plates at the end of this atlas.

\section{Tidal Elevation}

\section{Tidal Character}

The character of the tide in a particular region can be described in terms of the ratio, $\mathrm{F}=\left(\mathrm{K}_{1}+\mathrm{O}_{1}\right) /\left(\mathrm{M}_{2}+\mathrm{S}_{2}\right)$. 


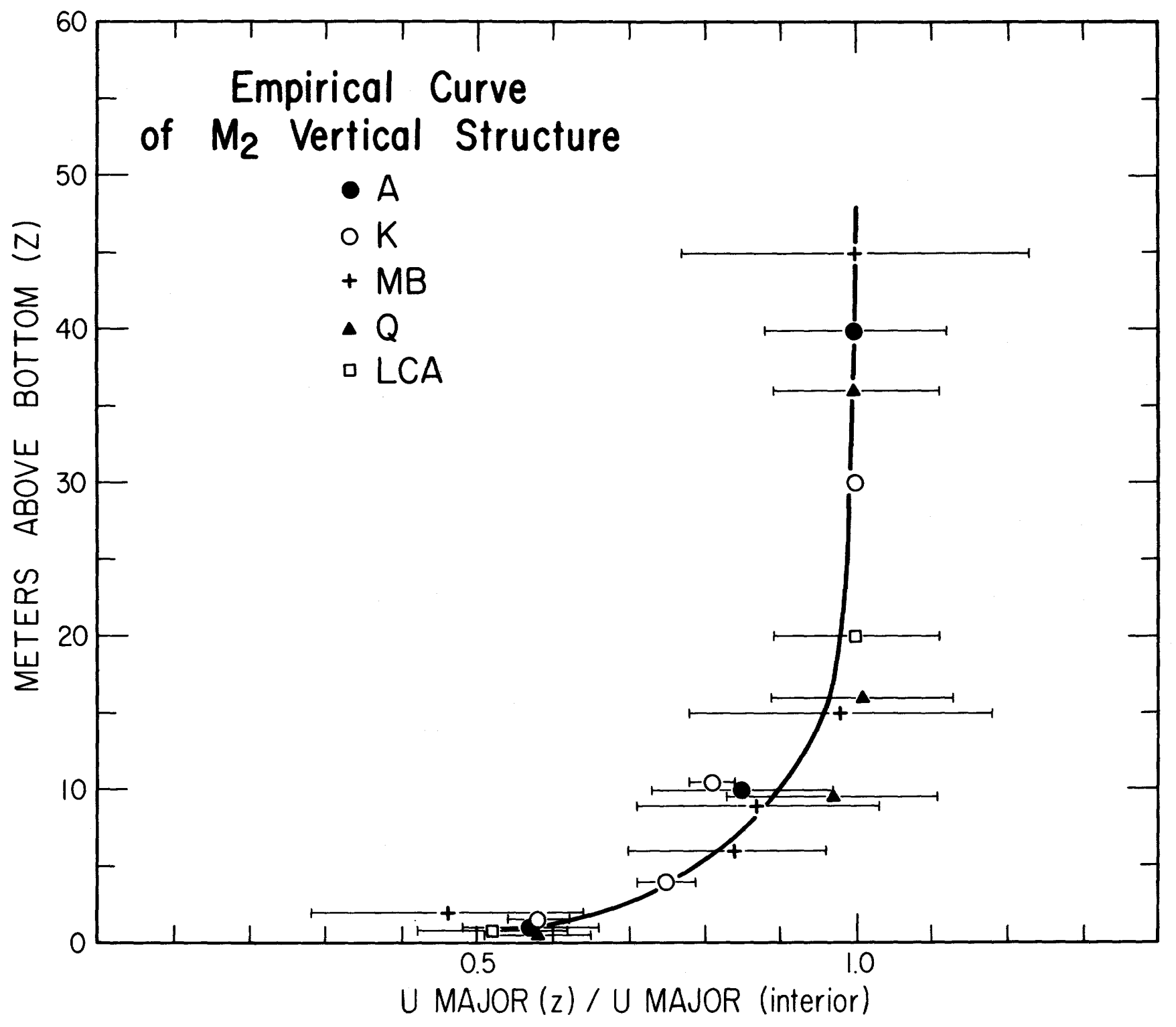

Figure 2. Vertical structure of normalized $M_{2}$ near-bottom current. Amplitude of the major axis of the $M_{2}$ tidal current ellipse at stations A, K, LCA, MB, and Q, normalized by the velocity observed at 40,30,20, 45, and 35 mab, respectively. The error bars are \pm 1 standard deviation and are zero for the observation at 30 mab (station K). For clarity, the plotted values at 1 mab have been slightly separated vertically. The solid line is a log curve from 0 to 15 mab, and is fit by eye above 15 mab. This empirical curve was used to extrapolate current measurements less than 20 mab to 1 mab (see plate 11).

Defant (1958) provides the following interpretations for F:

$\mathbf{F}=0.0-0.25$, semidiurnal;

$\mathrm{F}=0.25-1.50$, mixed, predominantly semidiurnal;

$\mathrm{F}=1.50-3.00$, mixed, predominantly diurnal; and

$\mathrm{F}>3.00$, diurnal.

Values of $F$ varied between 0.05 and 0.48 , indicating mixed but predominantly semidiurnal nature of the tide in this region (plate 3 ). Values of $F$ lower than 0.20 occurred mostly in the Gulf of Maine and the lowest values of $F$ were found at the head of the Bay of Fundy.

\section{$M_{2}$ Tide}

The coamplitude and cophase lines of the $\mathbf{M}_{2}$ semidiurnal tide (12.42 hours) shown in plate 4 are similar to the mean coamplitude and cophase lines published by Redfield (1953) for the Gulf of Maine and by Redfield (1958) and Swanson (1976) for the Middle Atlantic Bight. The phase $\left(\sim 350^{\circ}\right)$ and amplitude $(\sim 40 \mathrm{~cm})$ were nearly constant along the shelf break from Sable Island to Cape Hatteras. A feature not shown on earlier maps is an amplitude minimum $(30-40 \mathrm{~cm})$ centered over Nantucket 
Shoals and Georges Bank. The $\mathbf{M}_{\mathbf{2}}$ tide is predominantly a progressive, refracted wave across the shelf in the Georges Bank region, near co-oscillation in the Middle Atlantic Bight and on the Scotian Shelf, and near resonance with the $13.3 \mathrm{hr}$ natural period of the Gulf of Maine (Garrett, 1972 and 1974). The amplitude of the $\mathbf{M}_{2}$ increased from $40 \mathrm{~cm}$ at the shelf break to above $400 \mathrm{~cm}$ in the Bay of Fundy. Predictions by Redfield (1958) of the $\mathbf{M}_{2}$ amplitude and phase based on a damped, cooscillating model agree very well with observations southwest of Hudson Canyon and less well with those made in the transition region (from co-oscillation to progressive wave) northeast of Hudson Canyon $\left(71^{\circ} 30^{\prime} \mathrm{W}\right)$.

\section{$\mathbf{N}_{2}$ Tide}

The coamplitude and cophase lines of the $\mathrm{N}_{2}$ tide (12.66 hours) in plate 5 are similar to those of the $M_{2}$ tide in plate 4. However, the amplitude of the $\mathrm{N}_{2}$ tide is about 20 to 25 pecent of the amplitude of the $M_{2}$, slightly more than the mean $\mathrm{N}_{2} / \mathrm{M}_{2}$ ratio (19 percent) for the equilibrium tide (Schureman, 1941).

\section{S, Tide}

The coamplitude and cophase lines for the solar semidiurnal $S_{2}$ tide (12.00 hours) shown in plate 6 are also similar to those for the lunar semidiurnal $\mathbf{M}_{2}$ tide (plate 4). The observed amplitude ratio $S_{2} / M_{2}(0.20-0.25)$ is nearly the same as for the equilibrium tide $(0.21)$ over most of the continental Shelf, except in the Gulf of Maine and Bay of Fundy where it decreases to 0.10-0.18. Garrett (1972) has suggested that this decrease indicates that the Gulf of Maine and the Bay of Fundy are a single tidal system separate from the Continental Shelf.

\section{$K_{1}$ Tide}

The $\mathrm{K}_{1}$ tide (23.93 hours) has a distinct amphidromic point near Sable Island (plate 7), shown previously by Dohler (1954). Two possible amphidromic points are located near Cape Cod and Atlantic City, N.J. On the Scotian Shelf, the amplitude of the $K_{1}$ tide resembles a shelf wave; the amplitude decreases from 6-12 cm near the coast to 4-8 cm near the shelf break. The along-shelf phase velocity is about $50 \mathrm{~km} / \mathrm{hr}$. This is the same order of magnitude as the predicted phase speed of first-mode diurnal shelf waves along Vancouver Island $(10 \mathrm{~km} / \mathrm{hr}$, Crawford and Thomson, 1982), the Scottish Shelf $(25 \mathrm{~km} / \mathrm{hr}$, Cartwright and others, 1980), and the New England Shelf $(20 \mathrm{~km} / \mathrm{hr}$, Daifuku, 1981). In contrast, the $K_{1}$ tide in the Middle Atlantic Bight is dominated by a Kelvin wave with a phase speed of about $500 \mathrm{~km} / \mathrm{hr}$ (Daifuku, 1981; Daifuku and Beardsley,
1983). The $K_{1}$ period is about twice the 13.3 hour resonant period (Garrett, 1972) for the Gulf of Maine and thus there is only a modest amplification of the tidal elevation from $8 \mathrm{~cm}$ on Georges Bank to $16 \mathrm{~cm}$ at the head of the Bay of Fundy. In contrast, the near-resonant $\mathbf{M}_{2}$ amplitude increases 8-fold over the same area.

\section{O 1 Tide}

The major feature of the $\mathrm{O}_{1}$ tide ( 25.82 hours) is an amphidromic point near Sable Island (plate 8), similar to the $K_{1}$ tide. In this region of the Scotian Shelf, the amplitude of the $\mathrm{O}_{1}$ tide is constant across the shelf, rather than decreasing as the $K_{1}$ tide. In the Gulf of Maine, the amplitude of the $\mathrm{O}_{1}$ tide increases about 70 percent from the shelf break, and reaches a maximum of $12 \mathrm{~cm}$ approximately halfway into the Bay of Fundy. The amplitude then decreases toward the head of the Bay of Fundy. In contrast, on the shelf south of New England, the amplitude of the $\mathrm{O}_{1}$ tide decreases from a maximum of 7-8 cm seaward of the shelf break to $4-5 \mathrm{~cm}$ at the coast. This is similar to the idealized cotidal map published by Cartwright and others (1980) for the $K_{1}$ constituent on the Scottish Shelf where there is a superposition of a Kelvin wave and a shelf wave of approximately equal amplitude but out of phase. This superposition produced a virtual amphidrome, where the amplitude decreases rapidly toward the coast and the phase propagates backwards relative to the Kelvin wave.

\section{Tidal Current}

\section{$M_{2}$ Tidal Current}

The $\mathbf{M}_{2}$ tidal current is the strongest tidal constituent, and generally accounts for more than 80 percent of the total tidal variance represented by the five tidal constituents analyzed. The magnitude of the surface current is greater than $60 \mathrm{~cm} / \mathrm{s}$ over the shallow areas of Nantucket Shoals, on Georges Bank, south of the tip of Nova Scotia, and in the Bay of Fundy (plate 9). The largest tidal currents (in excess of $100 \mathrm{~cm} / \mathrm{s}$ ) are found on the Northeast Peak of Georges Bank and in the Bay of Fun$\mathrm{dy}$, corresponding to a tidal excursion in excess of $14 \mathrm{~km}$. The speed contours shown in the Gulf of Maine are based on only three stations in the northwest corner of the Gulf and a few along the northern edge of Georges Bank; the contours were drawn assuming that speeds are inversely proportional to water depth and, thus, decrease rapidly northward from the shallow water on the crest of Georges Bank to the deeper water of the Gulf of Maine. A small, mid-shelf maximum of the $\mathbf{M}_{2}$ surface current occurs in the Middle Atlantic Bight, where UMAJOR is slightly greater than $15 \mathrm{~cm} / \mathrm{s}$. 
The observed maximum cross-shelf tidal current in the region from Long Island southwestward to Cape Hatteras (plate 9) agrees well with the predictions by Redfield (1958) based on the simple canal theory of Sterneck (1915). Battisti and Clarke (1982a) have used a barotropic tidal model which includes rotation and friction to predict the cross-shelf and along-shelf tidal currents. The model results agree with available observations across the shelf southwest of Hudson Canyon and with the few near-shore observations that were used by Battisti and Clarke (1982b) south of Long Island. Brown (1984) has compared the tidal dynamics of the New England Shelf with those of Georges Bank and the Gulf of Maine and found that tidal differences are a result of different balances between the inertial force, coriolis force, and the pressure gradient in the along-isobath and cross-isobath directions.

The amplitude of the $M_{2}$ surface current along the shelf between the 60 - and $100-\mathrm{m}$ isobath from Northeast Channel southwestward into the Middle Atlantic Bight is shown in figure 3. The rapid decrease in tidal current speed just west of Nantucket Shoals may be responsible for the deposit of fine-grained sediment on the mid-shelf between $70^{\circ} 00^{\prime}$ and $71^{\circ} 30^{\prime} \mathrm{W}$. (Bothner and others, 1981; Twichell and others, 1981). This region of weak tidal currents and mixing coincides with the location of the coldest bottom water found on the mid- and outer
Continental Shelf in late spring (Houghton and others, 1982).

The orientation of the $\mathbf{M}_{2}$ ellipse is approximately perpendicular to the local isobaths (plates 9,10 ) in the Middle Atlantic Bight, the Gulf of Maine, Georges Bank, and on the Scotian Shelf. However, in the Northeast Channel and the Great South Channel and across Nantucket Shoals and a region southwest of Nova Scotia, the major axis of the tidal ellipse is approximately parallel to the local isobaths. Near the bottom the ellipse orientation is generally rotated slightly clockwise $\left(5^{\circ}-7^{\circ}\right)$ and the time of maximum speed is usually slightly earlier than at middepth (see stations $\mathrm{A}, \mathrm{K}, \mathrm{Q}$, and $\mathrm{MB}$ in table 5 , as examples). The amplitude of the major axis of the tidal currents (UMAJOR) decreases with depth but the ratio UMAJOR/UMINOR generally increases toward the bottom. The eccentricity $\left(e=\left(1-(\text { UMINOR/UMAJOR })^{2}\right)^{1 / 2}\right)$ of the tidal ellipses varies from 0.99 in regions with strong gradients of surface elevation (southern tip of Nova Scotia, Bay of Fundy, and east of Cape Cod at long $70^{\circ} 00^{\prime} \mathrm{W}$. and lat $41^{\circ} 30^{\prime} \mathrm{N}$.) to more nearly circular $(0.54)$ in a region (long $71^{\circ} 30^{\prime} \mathrm{W}$.) where the gradient of the $\mathrm{M}_{2}$ elevation is a minimum.

The $M_{2}$ tidal current rotates clockwise at 86 percent of the 301 instrument locations. Most of the stations at which the $\mathbf{M}_{2}$ tidal current rotated counterclockwise at all depths of observation are in the Gulf of Maine and Bay

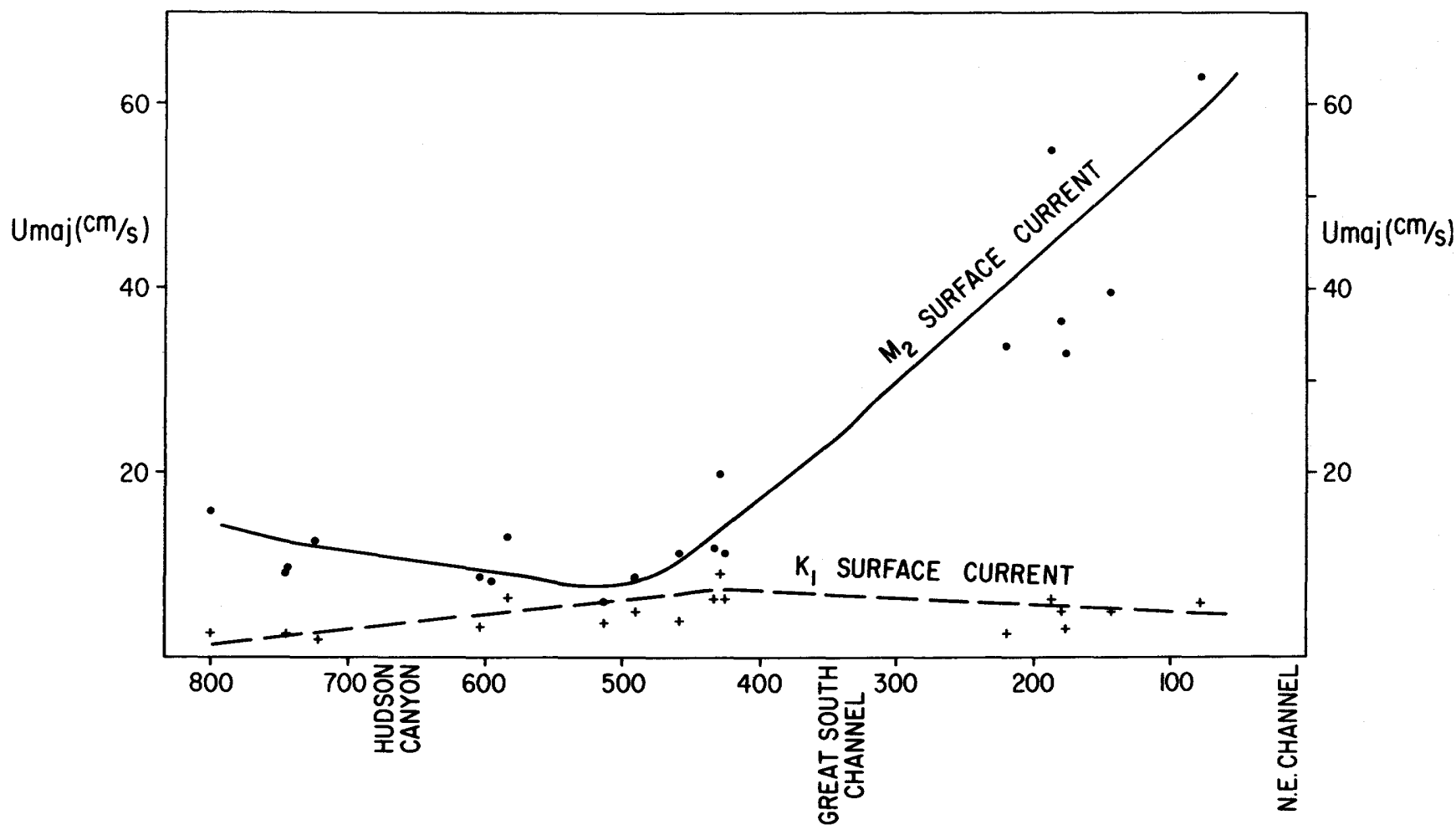

Figure 3. Longshelf variation in UMAJOR for the $M_{2}$ and $K_{1}$ constituents at stations located between the 60- and 100-m isobath from the Northeast Channel southward to station MB in the Middle Atlantic Bight. 
of Fundy with one (LTM) in Hudson Canyon. The tide at a few stations on the Scotian Shelf (S3, SS4, S8, SS12, $\mathrm{Cl}$, and C5), two stations in Lydonia Canyon (LCE and LCF), and two stations near Hudson Canyon (NJ4 and LT6) rotates clockwise near the surface and counterclockwise near the bottom.

The area of maximum $M_{2}$ bottom speed $(>40 \mathrm{~cm} / \mathrm{s})$ is on the Northeast Peak of Georges Bank (plate 11). The maximum near-bottom speeds of 7-8 $\mathrm{cm} / \mathrm{s}$ in the Middle Atlantic Bight are in a region just south of Hudson Canyon (plate 11). On the Scotian Shelf, maximum bottom speeds of $8-9 \mathrm{~cm} / \mathrm{s}$ are found about halfway across the shelf south of Halifax. Minimum bottom speeds of $3-4 \mathrm{~cm} / \mathrm{s}$ are in the same area as the minimum surface speeds, near lat $72^{\circ} \mathrm{W}$. between the 60 and $100-\mathrm{m}$ isobath.

The $\mathbf{M}_{2}$ tide over the Middle Atlantic and Scotian Shelf co-oscillates with the tide at the shelf break. This response is characterized by maximum off-shelf tidal current that occurs after maximum tidal elevation by about $90^{\circ}$ or 3 hours (plate 12), and by tidal elevation and current amplitude that are approximately uniform across the shelf. The $\mathbf{M}_{\mathbf{2}}$ tide on the southern flank of Georges Bank and Nantucket Shoals is a forced progressive wave. This response is characterized by a maximum northward flow into the Gulf of Maine, which is in phase with maximum elevation (plate 12). The tide in the Gulf of Maine is a forced standing wave with a $90^{\circ}$ phase difference between high water and maximum current.

The variability in amplitude and phase of the tidal currents is larger on the Continental Slope than on the Continental Shelf. Observations at three stations across the southern flank of Georges Bank clearly show the increase in variability toward deep water. At station A on the southern flank in water $85 \mathrm{~m}$ deep, the standard deviation of the phase was $2^{\circ}-3^{\circ}$; at station LCM in $120 \mathrm{~m}$ of water, the standard deviation of the phase was $7^{\circ}-10^{\circ}$; and at $\mathrm{LCI}$ on the slope in $250 \mathrm{~m}$ of water, the phase varied by $30^{\circ}-49^{\circ}$. A similar large phase uncertainty occurred at station MF $\left(16^{\circ}-38^{\circ}\right)$ on the Continental Slope in the Middle Atlantic Bight. There are also large phase differences between the surface and bottom currents on the Continental Slope at stations LCI $\left(92^{\circ}\right)$, LCJ $\left(198^{\circ}\right)$, LCK $\left(139^{\circ}\right)$, and MF $\left(95^{\circ}\right)$. These differences are probably due to the presence of significant baroclinic tides which are not found at shallow depths.

Several investigators have specifically analyzed their data for the baroclinic tide. At station SS4 in water $1,020 \mathrm{~m}$ deep on the Scotian Slope, the phase ranged from $2^{\circ}$ to $3^{\circ}$ at the surface to $21^{\circ}$ to $87^{\circ}$ at depths of 500 to $990 \mathrm{~m}$. The $\mathrm{M}_{2}$ tidal constants (table 5) for the slope stations SS1A, SS2A, SS4, SS5 and SS8 are almost identical to the baroclinic tidal constants reported by Petrie (1975). Petrie (1975) found that the baroclinic tide was the major tidal component on the Scotian Slope; the tide appeared to be generated at a depth of approximately
$200 \mathrm{~m}$ but was not observed within about $10 \mathrm{~km}$ of the shelf break. On the steep north flank of Georges Bank (station M1), Magnell (1980) found weak and variable tidal currents at mid-depth $(77 \mathrm{~m}$; see table 5) and stronger currents at deeper depths $(192 \mathrm{~m})$. He interpreted these observations as a baroclinic tide with a velocity node at about $80 \mathrm{~m}$.

$M_{2}$ Time Sequence

The sea-surface elevation and the tidal currents for the $\mathrm{M}_{2}$ lunar tide are shown in plates 13a-f at 2-hour intervals. High water occurs nearly simultaneously across the Middle Atlantic Bight at $0^{\text {hr }}$ Greenwich. At this time the current is weak and flows in the along-shelf direction toward the northeast (plate 13a). There is a strong flow across Georges Bank towards a sea-surface depression $(-40 \mathrm{~cm})$ near Boston and into a second depression $(-60 \mathrm{~cm})$ at the head of the Bay of Fundy.

After two hours, the sea-surface slopes down from the head of the Bay of Fundy across the Gulf of Maine and Georges Bank (plate 13b). However, flow is still into the Gulf of Maine and the Bay of Fundy and the sea surface continues to rise. The current on the Middle Atlantic and Scotian Shelves is then offshore, and sea level has fallen about 20 to $30 \mathrm{~cm}$ since $0^{\mathrm{hr}}$ Greenwich.

High water in the Bay of Fundy occurs just after $3^{\text {hr }}$ Greenwich and just before $4^{\mathrm{hr}}$ Greenwich at Boston. At $4^{\mathrm{hr}}$ Greenwich the current is weak in the western Gulf of Maine, just beginning to flow out of the Bay of Fundy and generally parallel to the local isobaths over Georges Bank (plate 13c). Flow continues to be offshore in the Middle Atlantic Bight and on the Scotian Shelf.

Low water (about $-60 \mathrm{~cm}$ ) occurs on the Middle Atlantic and Scotian Shelves at $6^{\text {hr }}$ Greenwich (plate 13d). The current flow along the shelf towards the southwest. Currents out of the Bay of Fundy and southward across Georges Bank reach a maximum at $6^{\text {hr }}$ Greenwich approximately three hours after high water in the Gulf of Maine.

A large northward slope in the sea surface develops in the Gulf of Maine region at $8^{\mathrm{hr}}$ Greenwich (plate 13e) as water continues to flow out of the Bay of Fundy and off the shelf. In the Middle Atlantic Bight and Scotian Shelf, the water is beginning to flow onshore and fill the depression left by low water two hours earlier.

Maximum sea-surface slope within the Gulf of Maine region occurs between $9^{\mathrm{hr}}$ and $10^{\mathrm{hr}}$ Greenwich, and at $10^{\mathrm{hr}}$ the elevation drops more than $4 \mathrm{~m}$ from $15-17 \mathrm{~cm}$ on the southern flank of Georges Bank to $-410 \mathrm{~cm}$ at the head of the Bay of Fundy. The currents are at the minimum amplitude on Georges Bank and oriented southwestward. The currents in the Middle Atlantic Bight and on the Scotian Shelf are onshore and reach a maximum at about $10^{\mathrm{hr}}$ Greenwich (plate $13 \mathrm{f}$ ). 
$\mathbf{N}_{2}$ Tidal Current

The ratio of the $\mathrm{N}_{2}$ UMAJOR amplitude to the $\mathrm{M}_{2}$ UMAJOR amplitude ranged from 0.20 to 0.30 . Because the $\mathrm{N}_{2} / \mathrm{M}_{2}$ ratio is nearly constant over most of the region, the pattern of the $N_{2}$ surface current (plate 14) is almost identical to the pattern of the $\mathbf{M}_{2}$ surface current (plate 9) with approximately one-fourth the amplitude.

The $\mathrm{N}_{2}$ and $\mathrm{M}_{2}$ constituents combine to produce a monthly (661.6-hour) modulation of the tidal current. This modulation is about 39 percent larger than the spring-neap modulation (see below) but has basically the same regional variation as that of the spring-neap variation shown in plate 16.

\section{$S_{2}$ Tidal Current}

The $S_{2}$ surface current is similar to the $M_{2}$ surface current pattern but the magnitude of the $S_{2}$ current is about 20 percent of the $M_{2}$ current (plate 15). The $S_{2}$ surface current is greater than $10 \mathrm{~cm} / \mathrm{s}$ on Georges Bank and in the Bay of Fundy and is $2-4 \mathrm{~cm} / \mathrm{s}$ in the Middle Atlantic Bight, in the Gulf of Maine, and on the Scotian Shelf. Like the $M_{2}$ currents, the major axis of the $S_{2}$ tidalcurrent ellipse is generally oriented perpendicular to the local isobaths, except south of Nantucket Shoals where the major axis of the ellipse is parallel to the local isobaths.

The solar $S_{2}$ tide combines with the lunar $\mathbf{M}_{2}$ tide to produce a spring-neap cycle with a period of 354.6 hours. The amplitude of the spring-neap current modulation is typically 15 to 20 percent of the $M_{2}$ amplitude (plate 16 ). The modulation amplitude is largest (30-40 percent of $M_{2}$ ) along the shelf break where the $M_{2}$ current speed is small and is nearly constant (15-20 percent) in a band midway across most of the shelf and into the Bay of Fundy. The modulation amplitude is a minimum in the area of strong $\mathbf{M}_{2}$ currents just east of Cape Cod (long $71^{\circ} 00^{\prime} \mathrm{W}$., lat $41^{\circ} 30^{\prime} \mathrm{N}$.).

\section{K Tidal Current}

The magnitude of the $K_{1}$ surface current is a maximum of $10 \mathrm{~cm} / \mathrm{s}$ over the area adjacent to Nantucket Shoals (long $69^{\circ}-70^{\circ} \mathrm{W}$., plate 17 ). A secondary maximum of $8 \mathrm{~cm} / \mathrm{s}$ occurs just south of the tip of Nova Scotia (approximately long $66^{\circ} \mathrm{W}$.). Note that the 4 and $6 \mathrm{~cm} / \mathrm{s}$ contours in plate 17 are drawn showing two distinct maxima separated by the Northeast Channel. The two maxima are separated by observations at three stations in the Northeast Channel made at depths greater than $100 \mathrm{~m}$ below the surface (although only one of these stations is shown in plate 17). The magnitude of the $K_{1}$ surface current is nearly constant $(2 \mathrm{~cm} / \mathrm{s})$ across most of the shelf southwest of Hudson Canyon and the lowest values $(<1.0 \mathrm{~cm} / \mathrm{s})$ are in the Gulf of Maine. No current observations were made around Sable Island, and thus no data are available to define the current associated with the strong amphidromic point observed for the $K_{1}$ tidal elevation in this region. There is a suggestion of three amphidromic points where successive cophase lines radiate from a central area. One point is near the southern tip of Nova Scotia, one point is off Nantucket Island (long $70^{\circ} \mathrm{W}$.), and one is near Atlantic City, N.J.

The $K_{1}$ tidal current rotates clockwise at 79 percent of the 266 instrument locations (plate 18, table 8). Like that of the $M_{2}$, counterclockwise rotation of the $K_{1}$ tidal current is observed mostly at stations in the Gulf of Maine and the Bay of Fundy. At several stations on the Scotian Shelf and near Lydonia Canyon the $\mathbf{K}_{1}$ tide also rotates clockwise at the surface and counterclockwise near the bottom. The $K_{1}$ tidal ellipses are oriented generally parallel to the isobaths on the Continental Shelf and nearly perpendicular to the isobaths in deeper water on the slope off Atlantic City, N.J., near Lydonia Canyon, and south of Halifax, Nova Scotia (plate 18). The $K_{1}$ tidal ellipses south of Halifax are nearly linear with eccentricities of 0.90 to 0.99 .

The uncertainty in the amplitude, orientation, and phase is large $\left(32^{\circ}-36^{\circ}\right)$ for the $K_{1}$ tide, and thus no significant relationship between these ellipse parameters and depth can be determined. The magnitude of the axis of the current ellipse generally decreases with depth; most exceptions are not significant due to the large uncertainty in the $K_{1}$ tidal current.

The $K_{1}$ tidal elevation leads the current by $60^{\circ}-120^{\circ}$ over the entire Continental Shelf from Cape Hatteras to Sable Island (plate 19). The phase difference does not vary significantly from $90^{\circ}$, given the relatively large variability $\left( \pm 36^{\circ}\right)$ in the phase of the $K_{1}$ current (table 2).

The variation and magnitude $(4-7 \mathrm{~cm} / \mathrm{s})$ of the $\mathrm{K}_{1}$ tidal-current velocities over the Scotian Shelf south of Halifax are the same order of magnitude as the diurnal shelf-wave velocities of $6-10 \mathrm{~cm} / \mathrm{s}$ computed by Cartwright and others (1980) for the west coast of Scotland, and velocities of $10-13 \mathrm{~cm} / \mathrm{s}$ predicted by Crawford and Thomson (1982) for the west coast of Vancouver Island. Although shelf waves are sensitive to forcing and to specific shelf topography, these similar current speeds suggest that the $K_{1}$ tidal current on the Scotian Shelf might be modeled as a shelf-wave. This suggestive shelf wave character for the $K_{1}$ currents on the Scotian Shelf has been modeled in more detail by Daifuku and Beardsley (1983) for the Middle Atlantic Bight.

\section{O, Tidal Current}

The amplitude of the $O_{1}$ surface current is about 72 percent of the amplitude of the $K_{1}$ surface current for the 
observations on the Scotian Shelf, Georges Bank, and in the Gulf of Maine (plate 20). This is essentially identical to the amplitude ratio $\left(\mathrm{O}_{1} / \mathrm{K}_{1}\right)$ for the equilibrium tide $(0.71)$. In contrast, the amplitude of the $O_{1}$ surface current in the region from Nantucket Shoals to Cape Hatteras is about 90 percent of the $K_{1}$ surface current although there was more variability $( \pm 46$ percent compared to \pm 26 percent on the Scotian Shelf, Georges Bank and Gulf of Maine). The magnitude of the $O_{1}$ surface current has the same general spatial pattern as the $K_{1}$ surface current. There are two current maxima of $6 \mathrm{~cm} / \mathrm{s}$, one centered over Nantucket Shoals ( $\sim$ long $71^{\circ} \mathrm{W}$.) and one south of Yarmouth, Nova Scotia ( long $65^{\circ} \mathrm{W}$.). Over Georges Bank, between these two maxima, is a region of reduced current amplitude and increased ellipse eccentricity. Over the remainder of the shelf, southwest of Nantucket Shoals, the magnitude of the $O_{1}$ surface current was nearly constant $(\sim 2 \mathrm{~cm} / \mathrm{s})$.

The along-shelf and cross-shelf variation of the $O_{1}$ current across the Scotian Shelf south of Halifax is similar to the $K_{1}$ currents although reduced in amplitude, and suggests a diurnal shelf wave propagating southwestward. The phase over Georges Bank suggests a progressive wave travelling across the bank into the Gulf of Maine. The rotation and orientation of the $\mathrm{O}_{1}$ ellipses is nearly the same as that described for the $K_{1}$ current (plate 21). The eccentricity of the $O_{1}$ ellipses was generally about 6 percent greater than that of the $K_{1}$ ellipses.

\section{SUMMARY}

The five constituents $\left(M_{2}, N_{2}, S_{2}, K_{1}\right.$, and $\left.O_{1}\right)$ account for more than 80 percent of the total variance of currents in the Georges Bank region, 30 to 40 percent in the Middle Atlantic Bight, and about 5 percent over the Continental Slope. These five constituents also account for more than 93 percent of the variance in tidal elevation which occurs at periods between 2 and 33 hours.

Semidiurnal tides characterize the water over the Continental Shelf from Cape Hatteras to Laurentian Channel, and mixed but predominantly semidiurnal tides are typical over the Continental Slope. The Gulf of Maine and Bay of Fundy system is near resonance with the frequency of the $M_{2}(12.42 \mathrm{hr})$ tidal forcing which causes large tidal elevations of 100 to $400 \mathrm{~cm}$ and large tidal currents of 70 to $100 \mathrm{~cm} / \mathrm{s}$ in the Bay of Fundy. Large $M_{2}$ tidal currents of 70 to $100 \mathrm{~cm} / \mathrm{s}$ also occur over Nantucket Shoals, Georges Bank, and Browns Bank along the seaward entrance to the Gulf of Maine. The semidiurnal tide elevation, however, is a minimum of 30 to $40 \mathrm{~cm}$ over most of this same region, which is a transition zone between an incident progressive, ocean wave and a standing wave in the Gulf of Maine. The Middle Atlantic and Scotian Shelves are outside the influence of the resonant Gulf of Maine system and are in cooscillation with the ocean tide. The $\mathbf{M}_{2}$ tidal elevation amplitude is 50 to $60 \mathrm{~cm}$ over the shelf and the tidal currents are 10 to $15 \mathrm{~cm} / \mathrm{s}$.

Observations at five stations on the Continental Shelf were used to construct an empirical curve which shows that the $\mathbf{M}_{2}$ tidal current at $1 \mathrm{mab}$ is approximately 50 percent of the midwater current amplitudes.

The $S_{2}(12.00 \mathrm{hr})$ and $N_{2}(12.66 \mathrm{hr})$ tidal elevations and currents are about 20 and 25 percent, respectively, of the corresponding $\mathbf{M}_{2}$ values over the entire Continental Shelf.

The diurnal tides, $\mathrm{K}_{1}(23.93 \mathrm{hr})$ and $\mathrm{O}_{1}(25.82 \mathrm{hr})$, have distinct amphidromic points near Sable Island, less distinct amphidromes in the Middle Atlantic Bight, and seem to be a complex combination of Kelvin and shelf waves. Tidal elevation amplitudes for both diurnal constituents range between 3 and $15 \mathrm{~cm}$ over the Continental Shelf. The diurnal tidal currents vary between 1 and $10 \mathrm{~cm} / \mathrm{s}$ with the largest currents in the vicinity of Nantucket Shoals and Browns Bank.

\section{REFERENCES CITED}

Aubrey, D., Twichell, D. C., and Pfirman, S., 1982, Holocene sedimentation off Nauset Inlet, Cape Cod, Mass.: Marine Geology, v. 47, p. 243-259.

Battisti, D. S., and Clarke, Allan, 1982a, A simple method for estimating barotropic tidal currents on continental margins with specific application to the $\mathbf{M}_{\mathbf{2}}$ tide off the Atlantic and Pacific coasts of the United States: Journal of Physical Oceanography, v. 12, p. 8-15.

$1982 b$, Estimation of nearshore tidal currents on nonsmooth continental shelves: Journal of Geophysical Research, v. 87, no. C10, p. 7873-7878.

Beardsley, R. C., Boicourt, W. C., Huff, L. C., McCullough, J. R., and Scott, J., 1981, CMICE: A near surface current meter intercomparison experiment: Deep-Sea Research, v. 28A, no. 12, p. 1577-1603.

Beardsley, R. C., Boicourt, W. C., Huff, L. C., and Scott, J., 1977a, A current meter intercomparison experiment conducted off Long Island in Feb.-March 1976: Woods Hole, Mass., Woods Hole Oceanographic Institution Technical Report 77-62, 123 p.

Beardsley, R. C., Mofjeld, H. O., Wimbush, M., Flagg, C. N., and Vermersch, J. A., Jr., 1977b, Ocean tides and weatherinduced bottom pressure fluctuations in the MiddleAtlantic Bight: Journal of Geophysical Research, v. 82, no. 21 , p. 3175-3182.

Beaumont, C., and Boutilier, R., 1978, Tidal loading in Nova Scotia; results from improved ocean tide model: Canadian Journal of Earth Science, v. 15, p. 981-993.

Bothner, M. H., Spiker, E. C., Johnson, P. P., Rendigs, R. R., and Aruscavage, R. J., 1981, Geochemical evidence for modern sediment accumulation on the Continental Shelf off southern New England: Journal of Sedimentary Petrology, v. 51, p. 281-292. 
Brown, W. S., 1984, A comparison of Georges Bank, Gulf of Maine, and New England Shelf tidal dynamics: Journal of Physical Oceanography, v. 14, p. 145-167.

Brown, W. S., Munk, W. H., Snodgrass, F., Mofjeld, H. O., and Zelter, B., Jan. 1975: MODE Bottom Experiment: Journal of Physical Oceanography, v. 5, no. 1, p. 75-85.

Butman, B., Beardsley, R. C., Magnell, B., Frye, D., Vermersch, J. A., Schlitz, R., Limeburner, R., Wright, W. R., and Noble, M. A., 1982, Recent observations of the mean circulation on Georges Bank: Journal of Physical Oceanography, v. 12, p. 569-591.

Butman, B., and Folger, D. W., 1979, An instrument system for long-term sediment transport studies on the continental shelf: Journal of Geophysical Research, v. 84, p. 1215-1220.

Butman, B., Noble, M. A., Chapman, D. C., and Beardsley, R. C., 1983, An upper bound for the tidally rectified current at one location on the southern flank of Georges Bank: Journal of Physical Oceanography, v. 13, no. 8, p. $1452-1460$.

Butman, B., and Moody, J. A., 1983, Observations of bottom currents and sediment movement along the U.S. east coast Continental Shelf during winter, Chapter 7, 60 p., in McGregor, B. A., ed., Environmental geological studies on the United States Mid and North Atlantic Outer Continental Shelf area, 1980-1982, Volume III. North Atlantic Region: Final Report to Minerals Management Service, Vienna, Va. Available from National Technical Information Service, accession no. PB848187954.

Canadian Hydrographic Service, 1966, Bay of Fundy data report on tidal and current survey, 1965: Bedford Institute of Oceanography, Data Series 66-2-D, unpublished manuscript.

1969a, Harmonic constants and associated data for Canadian tidal water: Tides and water levels: Marine Science Branch, Department of Energy, Mines, and Resources, Ottawa, v. 1, Atlantic coast.

$1969 \mathrm{~b}$, Bathymetric chart, Bay of Fundy to Gulf of St. Lawrence: Canadian Hydrographic Service, Ottawa, Canada.

Cartwright, D. E., Huthnance, J. M., Spencer, R., and Vassie, J. M., 1980, On the St. Kilda Shelf tide regime: Deep-Sea Research, v. 27a, p. 61-70.

Cartwright, D. E., Zetler, B. D., and Hamon, B. T., 1979, Pelagic tidal constants: International Association for the Physical Science of the Ocean (IAPSO), no. 30, $65 \mathrm{p}$.

Crawford, W. R., and Thomson, R. E., 1982, Continental shelf waves of diurnal period along Vancouver Island: Journal of Geophysical Research, v. 87, p. 9516-9522.

Daifuku, P. R., 1981, The diurnal tides on the northeast continental shelf off North America: Cambridge, Mass., Massachusetts Institute of Technology, unpublished Masters Thesis, $97 \mathrm{p}$.

Daifuku, P. R., and Beardsley, R. C., 1983, the $K_{1}$ tide on the continental shelf from Nova Scotia to Cape Hatteras: Journal of Physical Oceanography, v. 13, p. 3-17.

Defant, Albert, 1958, Ebb and flow: The tides of the earth, air, and water: Ann Arbor, Mich., University of Michigan Press, 122 p.
Dennis, R. E., and Long, E. E., 1971, A users guide to a computer program for harmonic analysis of data at tidal frequencies: U.S. National Oceanic and Atmospheric Administration (NOAA), Technical Report NOS41, 29 p.

Dohler, G., 1954, Tides in Canadian Waters: Canadian Hydrographic Service Marine Sciences Branch, Department of Energy, Mines, and Resources, Ottawa, cat. no. M54-966.

EG\&G, Environmental Consultants, 1976, Summary of oceanographic observations in New Jersey coastal waters near $39^{\circ} 28^{\prime} \mathrm{N}$. latitude and $74^{\circ} 15^{\prime} \mathrm{W}$. longitude during the period May 1974 through May 1975: A report to Public Service Gas Co., Newark, N.J.

1978, Summary of physical oceanographic observations near the site of the proposed Atlantic generating station offshore of Little Egg Inlet, New Jersey 1972 through 1976, January.

1979, Data report, Eulerian studies, April 1978: May 1979, Appendix B of Eleventh Quarterly Progress Report, July 1979.

1980, Analysis report, Appendix F of thirteenth quarterly progress report.

Farquharson, W. I., 1959, Causeway investigation Northumberland Strait report on tidal survey 1958: Canadian Hydrographic Service, Department of Mines and Technical Surveys, Ottawa, Canada.

Flagg, C. N., Magnell, B. A., Frye, D., Cura, J., McDowell, S., Scarlet, R., 1982, Interpretation of physical oceanography of Georges Bank, Final Report, v. 2 appendix C: Technical Report prepared for U.S. Department of Interior, Bureau of Land Management by EG\&G Environmental Consultants Report 82-B459, September. Available from National Technical Information Service, accession no. PB83149062.

Garrett, Christopher, 1972, Tidal resonance in the Bay of Fundy and Gulf of Maine: Nature, v. 238, p. 441-443.

1974, Normal modes of the Bay of Fundy and Gulf of Maine: Canadian Journal of Earth Science, v. 11, p. 549-556.

Garrett, Christopher, Keeley, J. R., and Greenberg, D. A., 1978, Tidal mixing versus thermal stratification in the Bay of Fundy and Gulf of Maine: Atmosphere-Ocean, v. 16, no. 4 , p. 403-423.

Garrett, Christopher, and Toulany, B., 1979, A variable depth Green's function for the shelf edge: Journal of Physical Oceanography, v. 9, no. 6, p. 1258-1272.

Goodrich, D. M., 1981, The tides of the New York Bight: Masters Thesis, Stony Brook, L. I., New York, State University of New York, $54 \mathrm{p}$.

Greenberg, D. A., 1975, Mathematical studies of tidal behavior in the Bay of Fundy: University of Liverpool, Ph.D. thesis, $139 \mathrm{p}$.

Haight, F. J., 1942, Coastal currents along the Atlantic coast of the United States: Coast and Geodetic Survey, Special Publication no. $230,73 \mathrm{p}$.

Halpern, D., and Pillsbury, R. D., 1976, Influences of surface waves on subsurface current measurements in shallow water: Limnology and Oceanography, v. 21, p. 611-616.

Hopkins, T. S., and Garfield, N., III, 1981, Physical origins of Georges Bank water: Journal of Marine Research, v. 39, p. $465-500$. 
Houghton, R. W., Schlitz, R., Beardsley, R. C., Butman, B., and Chamberlain, J. L., 1982, The Middle Atlantic Bight Cold Pool: Evolution of the temperature structure during summer 1979: Journal of Physical Oceanography, v. 12, no. 10, p. 1019-1029.

Lobecker, R. H., Higley, P. D., and Cook, D. O., 1978, A buoy system for acquiring, processing and telemetering real time OCS oceanographic data: Offshore Technology Conference, Proceedings, Houston, 11, p. 1133-1139 [OTC 3178].

Loder, J., 1980, Topographic rectification of tidal currents on the sides of Georges Bank: Journal of Physical Oceanography, v. 10, p. 1399-1416.

Magnell, B. A., Spiegel, S. L., Scarlet, R. I., and Andrews, J. B., 1980, The relationship of tidal and low-frequency currents on the north slope of Georges Bank: Journal of Physical Oceanography, v. 10, no. 8, p. 1200.

May, P. W., 1979, Analysis and interpretation of tidal currents in the coastal boundary layer: Cambridge, Mass., Massachusetts Institute of Technology, Ph.D. thesis, 200 p.

Mayer, D., 1982a, Circulation in the Hudson Shelf Valley MESA Physical oceanographic studies in New York Bight, Part I: Journal of Geophysical Research, v. 87, no. c12, p. 9563-9578.

$1982 \mathrm{~b}$, The structure of circulation, MESA physical oceanographic studies in New York Bight, Part II: Journal of Geophysical Research, v. 87, no. 12, p. 9579-9588.

Mayer, D. A., Hansen, D. V., and Ortman, D. A., 1979, Longterm current and temperature observations on the Middle Atlantic Shelf: Journal of Geophysical Research, v. 84, no. C4, p. 1776-1792.

Moody, J. A., and Butman, B., 1980, Semidiurnal bottom pressure and tidal currents on Georges Bank and in the Mid-Atlantic Bight: U.S. Geological Survey Open-File Report 80-1137, 22 p.

Moody, J. A., and Redfield, A. C., 1977, Harmonic analysis of the tides in Nantucket and Vineyard Sound: Woods Hole, Mass., Woods Hole Oceanographic Institution Contribution no. 3616.

Munk, W. H., and Cartwright, D. E., 1966, Tidal spectroscopy and prediction: Philosophical Transactions of the Royal Society of London, v. 259, no. 1105, p. 533-581.

National Ocean Service, Standard harmonic constants for prediction available for certain stations from Office of Oceanography and Marine Science, Tide and Current Prediction Section, National Oceanic and Atmospheric Administration, Rockville, M.D.

Noble, M. A., Butman, B., and Williams, E., 1983, On the longshelf structure and dynamics of subtidal currents on the eastern United States Continental Shelf: Journal of Physical Oceanography, v. 13, p. 2125-2147.

Patchen, R. C., Long, E. E., and Parker, B. B., 1976, Analysis of current meter observations in the New York Bight Apex, August 1973-June 1974: National Oceanic and Atmospheric Administration NOAA Technical Report ERL 386-MESA5.
Petrie, B., 1974, Surface and internal tides on the Scotian shelf and slope: Bedford, Nova Scotia, Dalhousie University, Ph.D. Thesis, $152 \mathrm{p}$.

$1975, M_{2}$ surface and internal tides on the Scotian Shelf and slope: Journal of Marine Research, p. 303-322.

Petrillo, A. F., 1981, Southern New England coastal sea bottom pressure dynamics: Narrangansett, R.I., University of Rhode Island, Masters Thesis, $168 \mathrm{p}$.

Redfield, A. C., 1950, The analysis of tidal phenomena in narrow embayments: Papers in Physical Oceanography and Meteorology, v. 11, no. 4, p. 1-36.

1953, Interference phenomena in the tides of the Woods Hole region: Journal of Marine Research, v. 12, p. 121-140.

1958, The influence of the continental shelf on the tides of the Atlantic coast of the United States: Journal of Marine Research, v. 17, p. 432-448.

1962, A practical portable tide gage: Limnology and Oceanography, v. 7, p. 262-265.

1980, The tides of the waters of New England and New York: Taunton, Mass., William S. Sullwold Publishing, Inc., $108 \mathrm{p}$.

Regal, R., and Wunsch, C., 1972, $M_{2}$ tidal currents in the western North Atlantic: Deep-Sea Research, v. 20, p. 493-502.

Schureman, P., 1941, Manual of harmonic analysis and prediction of tides: U.S. Department of Commerce, Coast and Geodetic Survey, Special Publication no. 98, 317 p.

Smith, P. C., 1983, The mean and seasonal circulation off southwest Nova Scotia: Journal of Physical Oceanography, v. 13, no. 6, p. 1034-1054.

Swanson, R. L., 1976, Tides; MESA New York Bight Atlas Monography 4: Albany, New York, New York Sea Grant Institute, $34 \mathrm{p}$.

Sterneck, R. V., 1915, Hydrodynamische Theorie der halbtagigen Gezeiten des Mittelmeeres: Sitzungsberichte der Akademie der Wissenschaften in Wien Mathematischnaturwissenschaftliche, Abt. II a, v. 124, p. 905-979.

Twichell, D. C., McClennen, C. E., and Butman, B., 1981, Morphology and processes associated with the accumulation of the fine-grained sediment deposit on the southern New England Shelf: Journal of Sedimentary Petrology, v. 51, no. 1, p. 296-280.

Uchupi, E., 1968, Atlantic continental shelf and slope of the United States, physiography: U.S. Geological Survey Professional Paper, No. 529-C, 30 p.

Vermersch, J. A., Beardsley, R. C., and Brown, W. S., 1979, Winter circulation in the western Gulf of Maine: Part 2. Current and pressure observations: Journal of Physical Oceanography, v. 9, p. 768, 771.

Wunsch, C., 1975, Internal tides in the ocean: Review of Geophysical and Space Physics, v. 13, p. 167-182.

Zetler, B., Munk, W. S., Mofjeld, H. O., Brown, W. S., and Dormer, F., 1975, MODE Tides: Journal of Physical Oceanography, v. 5, no. 3, p. 430-441. 
TABLES 3-9 

Table 3. Data references. Alphabetical listing of stations where tidal constants for current and/or elevation are tabulated, the region of the study area in which the station was located, the type of instrument used to make the current or pressure measurement, the method of data analysis, and the data source. The regions and station locations are shown in plate 1 . The tidal data are grouped by region in tables 4 through 9 . The region code is: SS, Scotian Shelf; GOM, Gulf of Maine; BF, Bay of Fundy; NEC, Northeast Channel; GB, Georges Bank; LC, Lydonia Canyon; GSC, Great South Channel; NS, Nantucket Shoals; NES, New England Shelf; MAB, Middle Atlantic Bight; SMAB, southern Mid-Atlantic Bight; and O, Oceanic. The type of instruments used to make the current or pressure observations are briefly described in Appendix I. The method of analysis (harmonic $=H$ and response $=\mathrm{R}$ ) is described in the text; $p$ means pressure, $c$ means current. The data source lists either a literature citation or an author of this atlas. If two station names have been used in the literature, the second name is listed in parentheses in the column labeled "source".

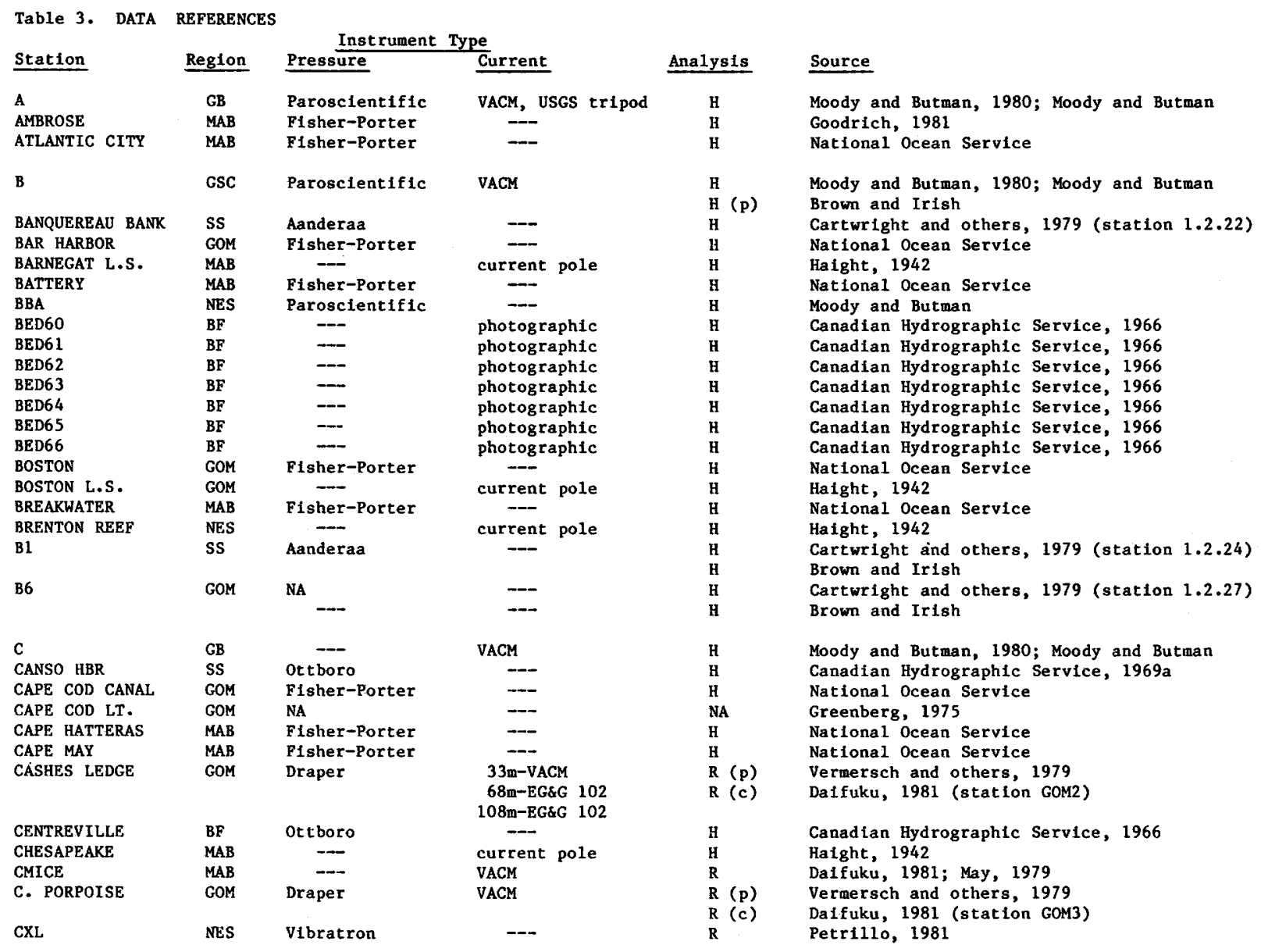


Table 3. Data references-Continued

Table 3. DATA REFERENCE--cont inued

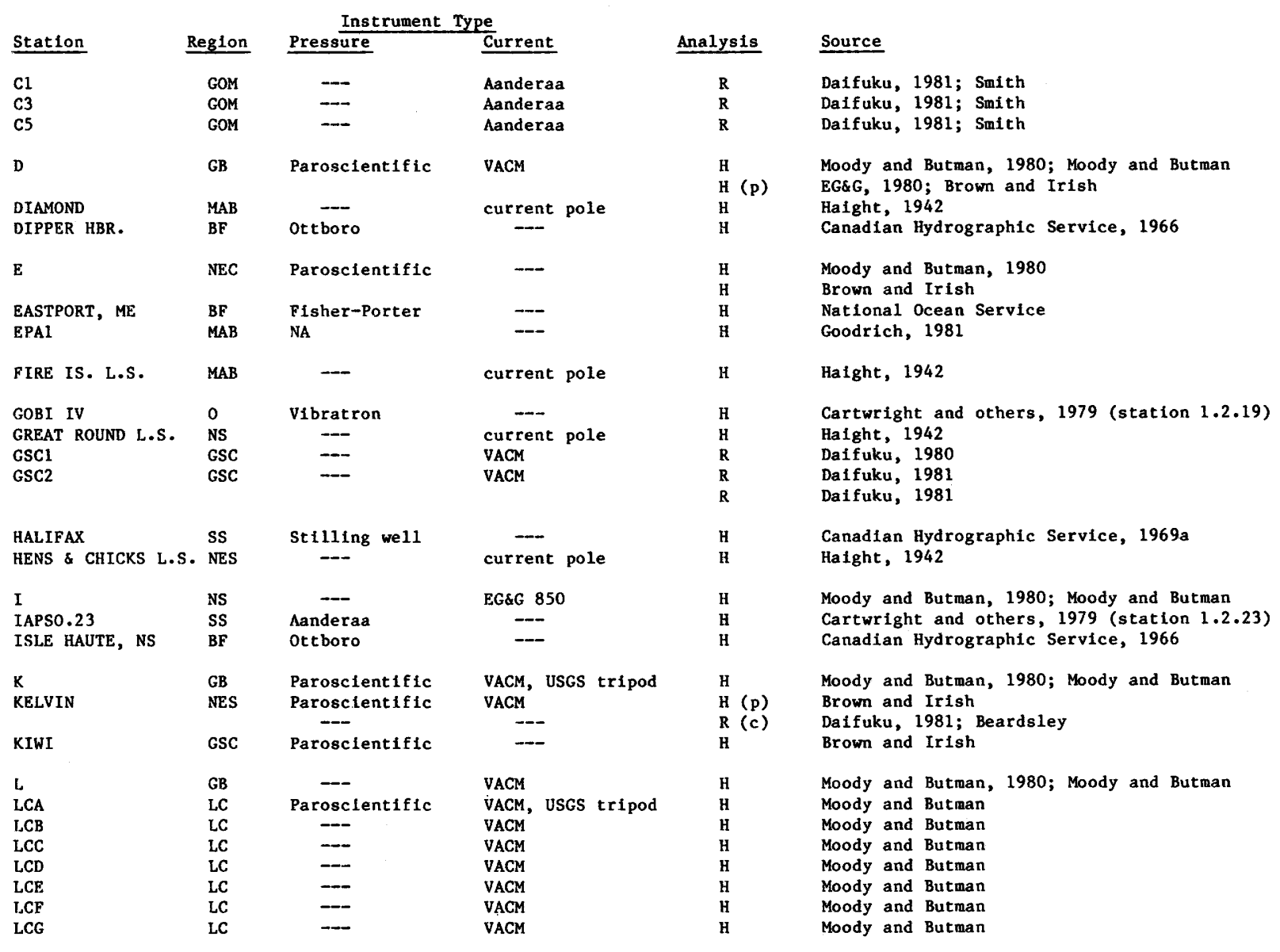


Table 3. Data references-Continued

Table 3. DATA REFERENCES--continued

\begin{tabular}{|c|c|c|c|c|}
\hline \multirow[b]{2}{*}{ Station } & \multirow{2}{*}{\multicolumn{3}{|c|}{ Instrument Type }} & \multirow{2}{*}{ inalysis } \\
\hline & & & Current & \\
\hline LCH & LC & -- & VACM & H \\
\hline LCI & LC & $-m$ & VACM & H \\
\hline LCJ & LC & -- & VACM & H \\
\hline LCK & LC & -- & VACM & H \\
\hline LCL & LC & Paroscientific & VACM & H \\
\hline LCM & LC & Paroscientific & VACM, USGS tripod & H \\
\hline LCN & LC & -- & VACM & H \\
\hline LCO & LC & Paroscientific & - & H \\
\hline L. EGG & MAB & bubbler & EGEG 102 & H \\
\hline LI1 & MAB & -- & Aanderaa & $\mathbf{R}$ \\
\hline LI2 & MAB & - & Aanderaa on spar & $\mathbf{R}$ \\
\hline LI3 & MAB & $-\infty$ & Aanderaa on spar & $\mathbf{R}$ \\
\hline LI4 & MAB & + & Aanderaa & $\mathbf{R}$ \\
\hline LOCKPORT & sS & Ottboro & -- & H \\
\hline LOUISBERG & SS & ottboro & $-\infty$ & H \\
\hline LTM & MAB & Paroscientific & Aanderaa & $\begin{array}{l}R(p) \\
H(p) \\
R(c)\end{array}$ \\
\hline LT2 & MAB & Paroscientific & $\begin{array}{l}\text { 3-m Aanderaa on spar } \\
15,23-m \text { Aanderaa }\end{array}$ & $\begin{array}{l}R \text { (p) } \\
H \quad(c) \\
R(c)\end{array}$ \\
\hline LT3 & MAB & - & Aanderaa & $\mathbf{R}$ \\
\hline LT4 & MAB & Paroscientific & $\begin{array}{l}\text { 3-m Aanderaa on spar } \\
24,44,51-m \text { Aanderaa }\end{array}$ & $\begin{array}{l}R(p) \\
H(p) \\
H(c)\end{array}$ \\
\hline LT5 & MAB & Paroscientific & Aanderaa & $\begin{array}{l}R \quad(p) \\
H(p) \\
H(c) \\
R(c)\end{array}$ \\
\hline LT6 & MAB & $-\infty$ & $\begin{array}{l}\text { 3-m Aanderaa on spar } \\
62-m \text { Aanderaa }\end{array}$ & $\mathbf{R}$ \\
\hline LT7 & MAB & $\rightarrow$ & Aanderaa & $\mathbf{R}$ \\
\hline M & GSC & -- & VACM & $\begin{array}{l}\mathbf{H} \\
\mathbf{R}\end{array}$ \\
\hline MA & MAB & Paroscientific & USGS tripod & H \\
\hline MAB & MAB & - & $\begin{array}{l}\text { 9-m Endeco(modif.) } \\
21,32-m \text { Aanderaa }\end{array}$ & $\mathbf{R}$ \\
\hline MARGARETV ILLE & BF & ottboro & -- & H \\
\hline MB & MAB & Paroscientific & VACM, USGS tripod & H \\
\hline MC & MAB & Paroscientific & USGS tripod & $\mathbf{H}$ \\
\hline MD & MAB & Paroscientific & USGS tripod & H \\
\hline ME & MAB & Paroscientific & USGS tripod & H \\
\hline MENEMSHA & NES & bubbler & -- & $\mathrm{H}$ \\
\hline MESA & MAB & Filloux & -- & $\mathbf{H}$ \\
\hline MESA7 & MAB & - & Aanderaa & $\mathbf{R}$ \\
\hline MESA9 & MAB & Filloux & & $\mathbf{R}$ \\
\hline MESA10 & MAB & Vibratron & --- & $\mathbf{R}$ \\
\hline MESA11 & MAB & vibratron & -- & $\mathbf{R}$ \\
\hline
\end{tabular}

Source

Moody and Butman

Moody and Butman

Moody and Butman

Moody and Butman

Moody and Butman

Moody and Butman

Moody and Butman

Moody and Butman

EG\&G, 1976

Mayer, 1982b

Mayer, $1982 b$

Mayer, 1982b

Mayer, 1982b

Canadian Hydrographic Service, 1969a

Canadian Hydrographic Service, 1969

Mof jeld; Goodrich

Brown and Irish

Mayer and others, .1979

Mof jeld; Brown and Irish

National Ocean Service

Mayer, 1982b

Daifuku, 1981 (station MESA3)

Mofjeld; Goodrich

Brown and Irish

National Ocean Service

Mof Jeld; Goodrich, 1981

Brown and Irish

National Ocean Service

Dalfuku, 1981 (station MESA5)

Mayer, 1982b

Mayer, 1982b

Moody and Butman, 1980; Moody and Butman; Dalfuku, 1981 (station GSC3)

Moody and Butman, 1980; Moody and Butman Dalfuku, 1981; Boicourt

Canadian Hydrographic Service, 1966

Moody and Butman, 1980; Moody and Butman Moody and Butman, 1980; Moody and Butman Moody and Butman, 1980; Moody and Butman Moody and Butman

Moody and Redfleld, 1977; Moody

Cartwright and others, 1979 (station 1.2.17); Mof jeld Dalfuku, 1981

Beardsley and others, 1977; Goodrich, 1981

Beardsley and others, 1977; Goodrich, 1981

Beardsley and others, 1977; Goodrich, 1981 
Table 3. Data references-Continued

Table 3. DATA REFERENCES--continued

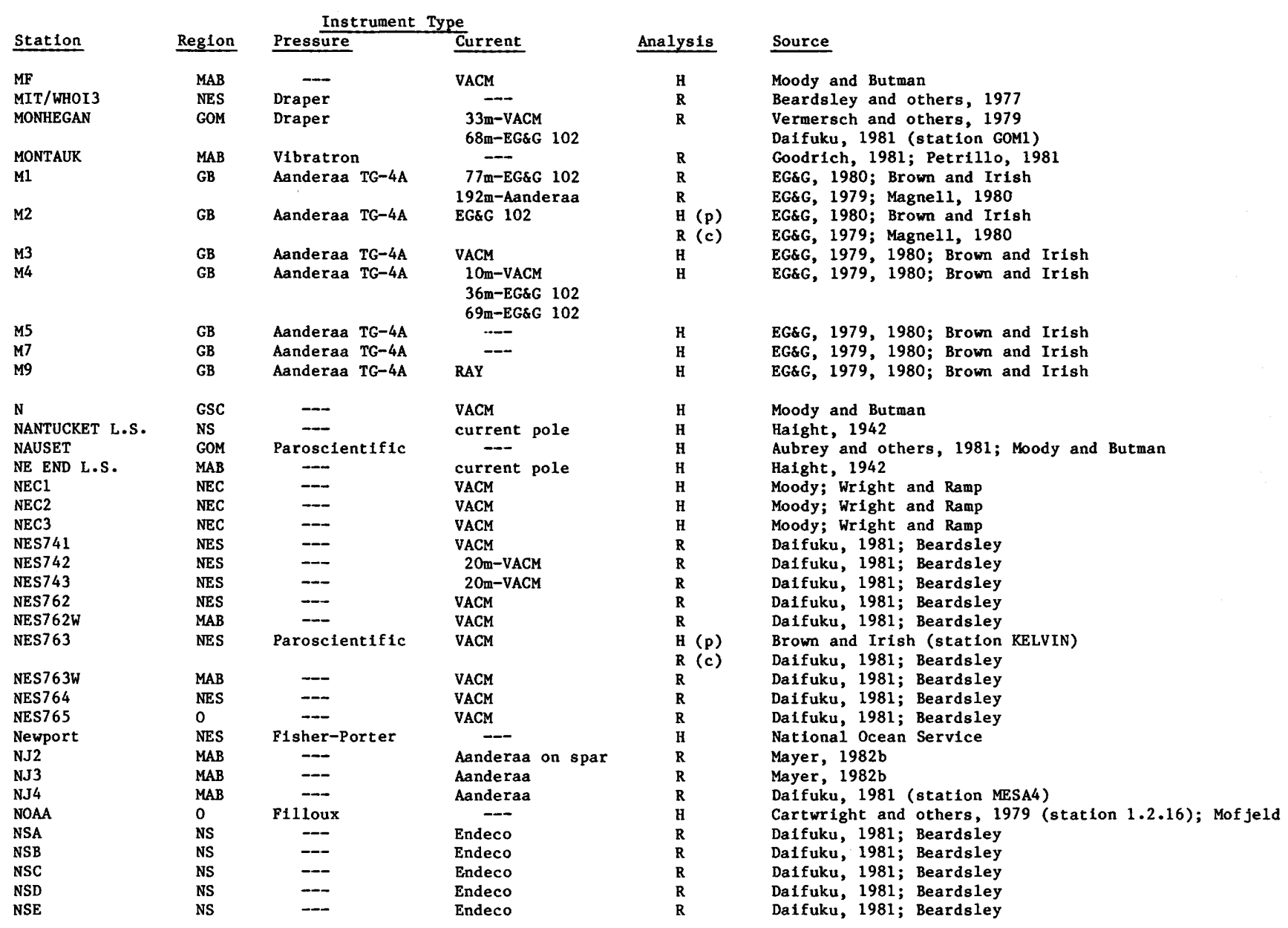


Table 3. Data references-Continued

Table 3. DATA REFERENCES--continued

\begin{tabular}{|c|c|c|c|c|c|}
\hline \multirow[b]{2}{*}{ Station } & \multicolumn{3}{|c|}{ Instrument Type } & \multirow[b]{2}{*}{ Analysis } & \multirow[b]{2}{*}{ Source } \\
\hline & Region & Pressure & Current & & \\
\hline NSFE 1 & NS & Paroscientific & VACM & $\begin{array}{l}H(p) \\
R(c)\end{array}$ & $\begin{array}{l}\text { Brown and Irish } \\
\text { Daifuku, } 1981\end{array}$ \\
\hline NSFE2 & NS & Paroscientific & VACM & $\begin{array}{l}\text { H (p) } \\
\text { R (c) }\end{array}$ & $\begin{array}{l}\text { Brown and Irish } \\
\text { Dalfuku, } 1981\end{array}$ \\
\hline NSFE3 & NS & -- & VACM & $\mathbf{R}$ & Datfuku, 1981; Beardsley \\
\hline NSFE4 & NS & Paroscientific & VACM & $\begin{array}{l}\text { H (p) } \\
R(c)\end{array}$ & $\begin{array}{l}\text { Brown and Irish } \\
\text { Daffuku, } 1981\end{array}$ \\
\hline NSFE5 & NS & Paroscientific & VACM & $\begin{array}{l}\text { R (p) } \\
R(c)\end{array}$ & $\begin{array}{l}\text { Brown and Irish } \\
\text { Daifuku, } 1981\end{array}$ \\
\hline NSPE6 & NS & -- & VACM & $\mathbf{R}$ & Daifuku, 1981 ; Beardsley \\
\hline OCEAN CITY, MD & MAB & Fisher-Porter & -- & $\mathbf{H}$ & National Ocean Service \\
\hline $\mathbf{P}$ & NES & Paroscientific & VACM, USGS tripod & $\mathrm{H}$ & Moody and Butman, 1980; Moody and Butman \\
\hline PICKET & NES & Paroscientific & -- & H & Brown and Irish \\
\hline PINKNEY PT. & GOM & ottboro & -- & $\mathrm{H}$ & Canadian Hydrographic Service, 1969a \\
\hline POLLOCK L.S. & NS & -- & current pole & $\mathrm{H}$ & Haight, 1942 \\
\hline PORTLAND, ME & GOM & Fisher-Porter & $-\infty$ & $\mathbf{H}$ & National Ocean Service \\
\hline PORT MAITLAND & GOM & Ottboro & -- & $\mathbf{H}$ & Canadian Hydrographic Service, 1969a \\
\hline PORTSMOUTH, NH & GOM & Fisher-Porter & -- & H & National Ocean Service \\
\hline & GB & -- & Aanderaa & H & Moody; Ramp, Wright, Allen \\
\hline P2 & GB & -- & $\begin{array}{l}\text { VACM } \\
\text { 30m-Aanderaa }\end{array}$ & H & Moody; Ramp, Wright, Allen \\
\hline P3 & GB & -- & Aanderaa & H & Moody; Ramp, Wright, Allen \\
\hline P4 & GB & -- & VACM & $\mathbf{H}$ & Moody; Ramp, Wright, Allen \\
\hline P5 & GB & -- & VACM & $\mathbf{H}$ & Moody; Ramp, Wright, Allen \\
\hline P6 & GB & - & VACM & $\mathbf{H}$ & Moody; Ramp, Wright, Allen \\
\hline P11 & MAB & -- & Aanderaa & $\mathbf{R}$ & Mayer \\
\hline P12 & MAB & -- & Aanderaa & $\mathbf{R}$ & Mayer \\
\hline P22 & MAB & Paroscientific & --- & H & Mof jeld; Goodrich, 1981 \\
\hline P31 & MAB & Paroscientific & Aanderaa & $\begin{array}{l}R(p) \\
R(c)\end{array}$ & $\begin{array}{l}\text { Mof Jeld; Goodrich, } 1981 \\
\text { Mayer }\end{array}$ \\
\hline P32 & MAB & Paroscientific & NA & $\begin{array}{l}R(p) \\
R(c)\end{array}$ & $\begin{array}{l}\text { Mof jeld } \\
\text { Mayer }\end{array}$ \\
\hline Q & NS & Paroscientific & VACM, USGS tripod & $\mathbf{H}$ & Moody and Butman, 1980; Moody and Butman \\
\hline $\mathbf{R}$ & GsC & Paroscientific & VACM & H & Moody and Butman, 1980; Moody and Butman \\
\hline ROCKLAND, ME & GOM & Fisher-Porter & - & $\mathbf{H}$ & National Ocean Service \\
\hline REIKO-MAY & 0 & Hewlett-Packard & $-\infty$ & $\mathbf{R}$ & Brown and others, 1975: Zetler and others, 1975 \\
\hline $\mathbf{S}$ & NS & Paroscientific & --- & $\mathbf{H}$ & Moody and Butman \\
\hline SABLE IS. & sS & Ottboro & --- & Il & Canadian Hydrographic Service, 1969a \\
\hline SANDY HOOK & MAB & Fisher-Porter & -- & H & National Ocean Service \\
\hline SEAL IS. & GOM & ottboro & -- & H & Canadian Hydrographic Service, 1969a \\
\hline SHINNECOCK & MAB & bubbler & -- & $\mathrm{H}$ & National Dcean Service; Goodrich, 1981 \\
\hline
\end{tabular}


Table 3. Data references-Continued

Table 3. DATA REFERENCES--continued

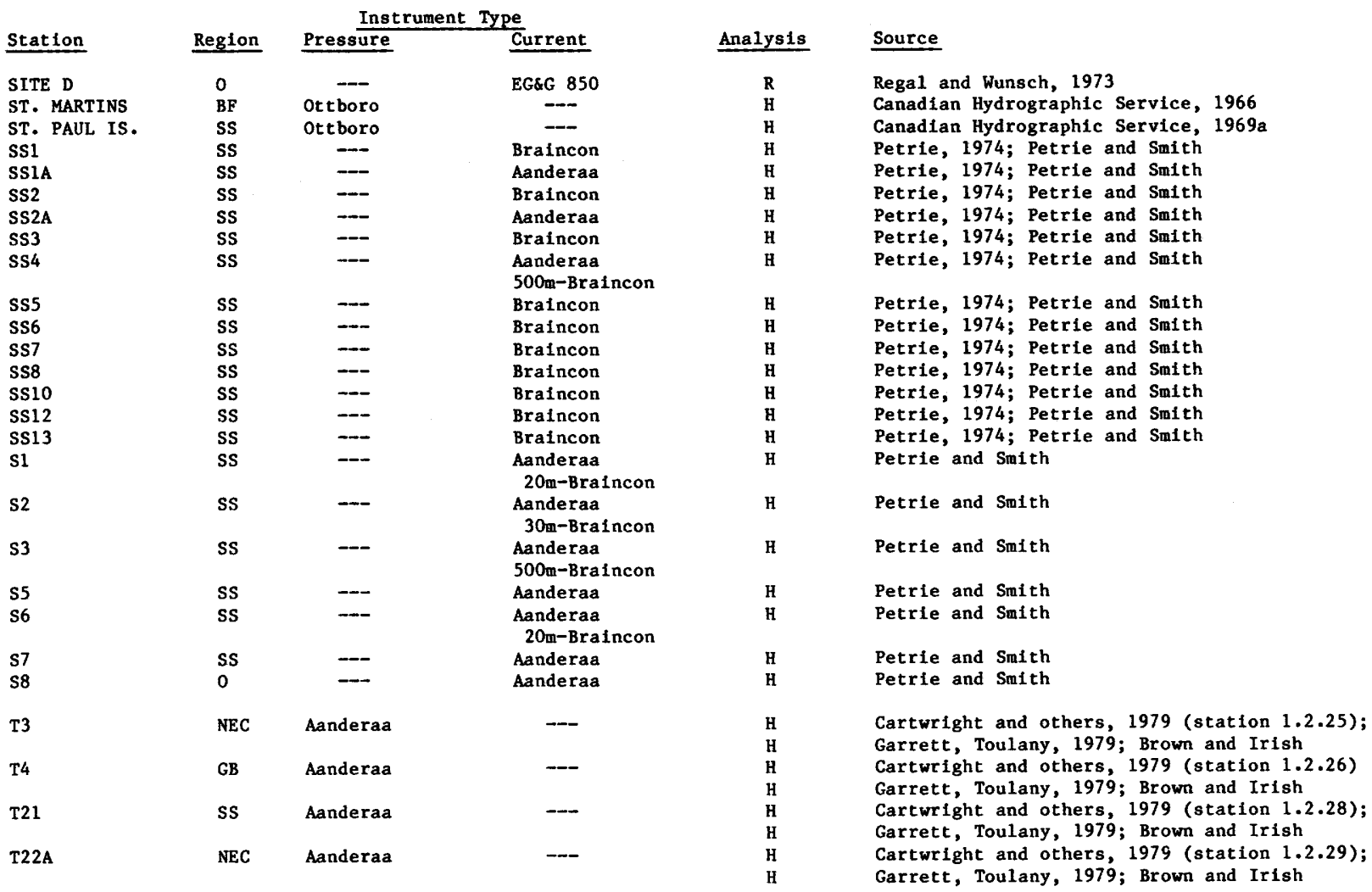


Table 3. Data references-Continued

Table 3. DATA REFERENCES--continued

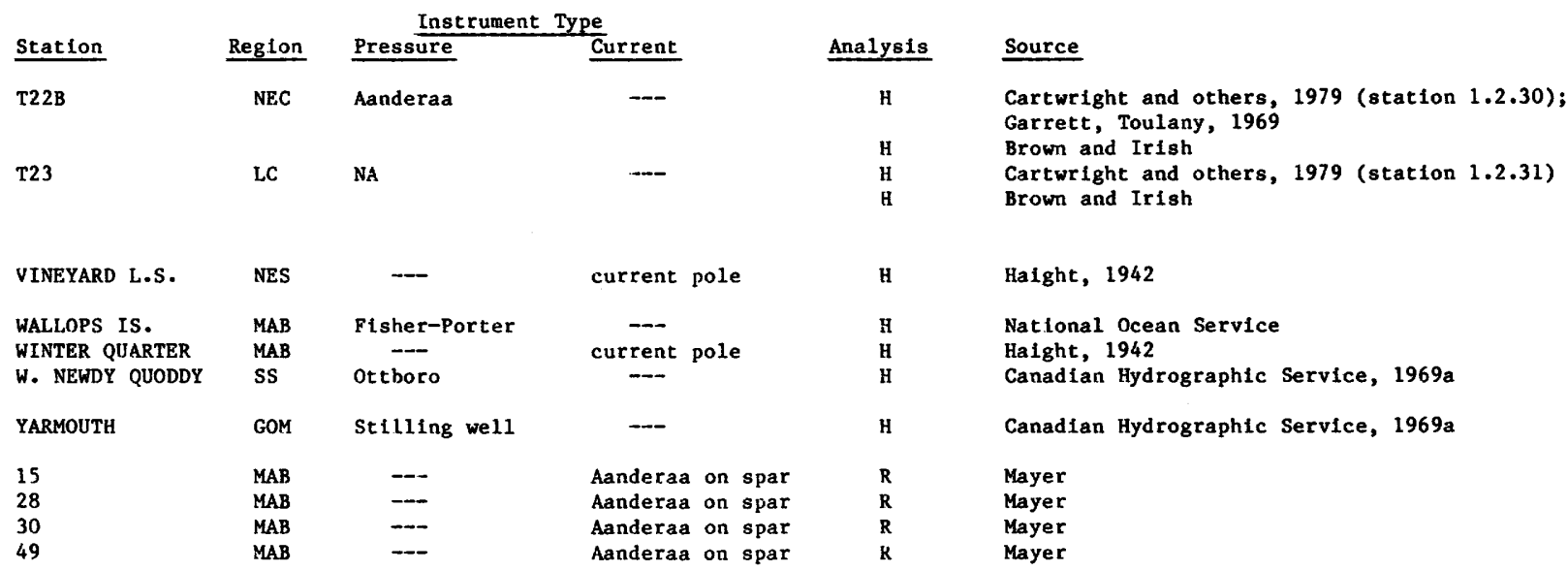


Table 4. Tidal constants for elevation. The stations are grouped by region (see plate 1), and are listed within each region from east to west. For each station, the latitude and longitude, the record length, the instrument depth, and the amplitude (in $\mathrm{cm}$ ) and phase (in ${ }^{\circ} \mathrm{Greenwich}$ ) for the $\mathrm{M}_{2}, \mathrm{~N}_{2}, \mathrm{~S}_{2} \mathrm{~K}_{1}$ and $\mathrm{O}_{1}$ constituents are tabulated. Where separate analysis was done by two different investigators, both are listed. The $( \pm)$ number which follows some constants is the standard deviation of the constants computed by the harmonic method for each data piece, or an estimate of the standard error where the constants have been determined by he response method (see Data Analysis for discussion).

\begin{tabular}{|c|c|c|c|c|c|c|c|c|c|c|c|c|c|}
\hline \multirow{2}{*}{$\begin{array}{c}\text { STATION } \\
\text { LAT. } \\
\text { LONG. }\end{array}$} & \multirow{2}{*}{$\begin{array}{l}\text { RECORD } \\
\text { LENGTH } \\
\text { (DAYS) }\end{array}$} & \multirow{2}{*}{$\begin{array}{l}\text { INSTR } \\
\text { DEPTH } \\
\text { (M) }\end{array}$} & \multirow{2}{*}{$\begin{array}{l}\text { ABOVE } \\
\text { BOTTOM } \\
\text { (M) }\end{array}$} & \multicolumn{2}{|c|}{ M2 } & \multicolumn{2}{|c|}{ N2 } & \multicolumn{2}{|c|}{ S2 } & \multicolumn{2}{|c|}{$\mathrm{K} 1$} & \multicolumn{2}{|c|}{01} \\
\hline & & & & $\begin{array}{l}\text { AMP } \\
\text { (CM) }\end{array}$ & $\begin{array}{c}\text { PHASE } \\
\text { (DEG-G) }\end{array}$ & $\begin{array}{l}\text { AMP } \\
\text { (CM) }\end{array}$ & $\begin{array}{l}\text { PHASE } \\
\text { (DEG-G) }\end{array}$ & $\begin{array}{l}\text { AMP } \\
\text { (CM) }\end{array}$ & $\begin{array}{c}\text { PHASE } \\
\text { (DEG-G) }\end{array}$ & $\begin{array}{l}\text { AMP } \\
\text { (CM) }\end{array}$ & $\begin{array}{l}\text { PHASE } \\
\text { (DEG-G) }\end{array}$ & $\begin{array}{l}\text { AMP } \\
(\mathrm{CM})\end{array}$ & $\begin{array}{c}\text { PHASE } \\
\text { (DEG-G) }\end{array}$ \\
\hline \multicolumn{14}{|l|}{ SCOTIAN SHELF } \\
\hline $\begin{array}{c}\text { BANQUEREAU* } \\
44^{\circ} 35^{\circ} \mathrm{N} . \\
57^{\circ} 41^{-\mathrm{W}} .\end{array}$ & * 36 & 64 & $\mathrm{NA}$ & 44.1 & 345 & 9.8 & 320 & 10.7 & 23 & 5.5 & 162 & 4.7 & 166 \\
\hline $\begin{array}{l}\text { ST. PAUL } \\
47^{\circ} 12^{-N} . \\
60^{\circ} 09^{-} \mathrm{W} .\end{array}$ & 730 & $\mathrm{NA}$ & $\mathrm{NA}$ & 30.8 & 366 & 6.4 & 343 & 10.1 & 47 & 8.5 & 295 & 9.1 & 266 \\
\hline $\begin{array}{l}\text { SABLE IS } \\
43^{\circ} 58^{-} \mathrm{N} . \\
59^{\circ} 48^{-} \mathrm{W} .\end{array}$ & 73 & $\mathrm{NA}$ & $\mathrm{NA}$ & 52.4 & 352 & 9.1 & 333 & 11.6 & 29 & 2.7 & 162 & 2.7 & 211 \\
\hline $\begin{array}{l}\text { LOUISBERG } \\
45^{\circ} 55^{-} \mathrm{N} . \\
59^{\circ} 58^{-} \mathrm{W} .\end{array}$ & 29 & $\mathrm{NA}$ & NA & 50.3 & 344 & 11.0 & 312 & 12.0 & 24 & 6.1 & 24 & 6.1 & 321 \\
\hline $\begin{array}{l}\text { CANSO HBR. } \\
45^{\circ} 20^{-} \mathrm{N} . \\
61^{\circ} 00^{-} \mathrm{W}\end{array}$ & 15 & $\mathrm{NA}$ & $\mathrm{NA}$ & 58.8 & 347 & 10.1 & 345 & 14.0 & 20 & 6.4 & 48 & 2.7 & 335 \\
\hline $\begin{array}{l}\text { N. NEWDY } \\
44^{\circ} 54^{-} \mathrm{N} . \\
62^{\circ} 19^{-} \mathrm{W} .\end{array}$ & 29 & $\mathrm{NA}$ & NA & 60.7 & 347 & 16.9 & 328 & 16.6 & 26 & 8.8 & 95 & 4.4 & 57 \\
\hline $\begin{array}{l}\mathrm{Bl}^{*} \\
42^{\mathrm{O}} 49^{-} \mathrm{N} . \\
63^{\circ} 12^{-} \mathrm{W} .\end{array}$ & $\begin{array}{l}63 \\
62\end{array}$ & $\begin{array}{l}226 \\
226\end{array}$ & $\begin{array}{l}\mathrm{NA} \\
\mathrm{NA}\end{array}$ & $\begin{array}{l}48.2 \\
48.3\end{array}$ & $\begin{array}{l}351 \\
350\end{array}$ & $\begin{array}{l}11.9 \\
12.5\end{array}$ & $\begin{array}{l}329 \\
323\end{array}$ & $\begin{array}{l}10.3 \\
11.0\end{array}$ & $\begin{array}{l}22 \\
24\end{array}$ & $\begin{array}{l}6.9 \\
6.7\end{array}$ & $\begin{array}{l}173 \\
172\end{array}$ & $\begin{array}{l}5.7 \\
5.4\end{array}$ & $\begin{array}{l}170 \\
177\end{array}$ \\
\hline $\begin{array}{l}\text { HALIFAX } \\
44^{\circ} 40^{-} \mathrm{N} \\
53^{\circ} 35^{-} \mathrm{W} .\end{array}$ & 365 & $\mathrm{NA}$ & $\mathrm{NA}$ & 63.0 & 349 & 14.3 & 329 & $14 \cdot 3$ & 21 & 10.3 & 121 & 4.8 & 93 \\
\hline $\begin{array}{l}\text { IAPSO.23* } \\
42^{\circ} 47^{\circ} \mathrm{N} \\
63^{\circ} 59^{-} \mathrm{W}\end{array}$ & 102 & 246 & NA & 49.2 & 350 & 10.8 & 330 & 11.7 & 20 & 6.9 & 172 & 5.9 & 176 \\
\hline $\begin{array}{l}\mathrm{T} 21^{*} \\
42^{\mathrm{o}} 37^{-} \mathrm{N} . \\
64^{\circ} 22^{-} \mathrm{W} .\end{array}$ & $\begin{array}{l}58 \\
\mathrm{NA}\end{array}$ & $\begin{array}{l}232 \\
232\end{array}$ & $\begin{array}{l}\mathrm{NA} \\
\mathrm{NA}\end{array}$ & $\begin{array}{l}49.0 \\
48.7\end{array}$ & $\begin{array}{l}357 \\
356\end{array}$ & $\begin{array}{l}11.6 \\
13.2\end{array}$ & $\begin{array}{l}335 \\
341\end{array}$ & $\begin{array}{l}10.3 \\
10.4\end{array}$ & $\begin{array}{l}23 \\
24\end{array}$ & $\begin{array}{l}6.8 \\
6.2\end{array}$ & $\begin{array}{l}170 \\
161\end{array}$ & $\begin{array}{l}5.4 \\
5.4\end{array}$ & $\begin{array}{l}179 \\
178\end{array}$ \\
\hline $\begin{array}{l}\text { LOCKPORT } \\
43^{\circ} 42^{-} \mathrm{N} \\
65^{\circ} 07^{-} \mathrm{W}\end{array}$ & 29 & NA & NA & 69.8 & 359 & 17.1 & 337 & 13.7 & 29 & 12.8 & 147 & 7.6 & 116 \\
\hline
\end{tabular}

Instrument type, method of analysis and source of data are 1isted alphabetically by stations in Table 3 .

* - Pressure data was converted to $\mathrm{cm}$ using $1 \mathrm{mb}=0.995 \mathrm{~cm}$.

NA - not available 
Table 4. Tidal constants for elevation-Continued

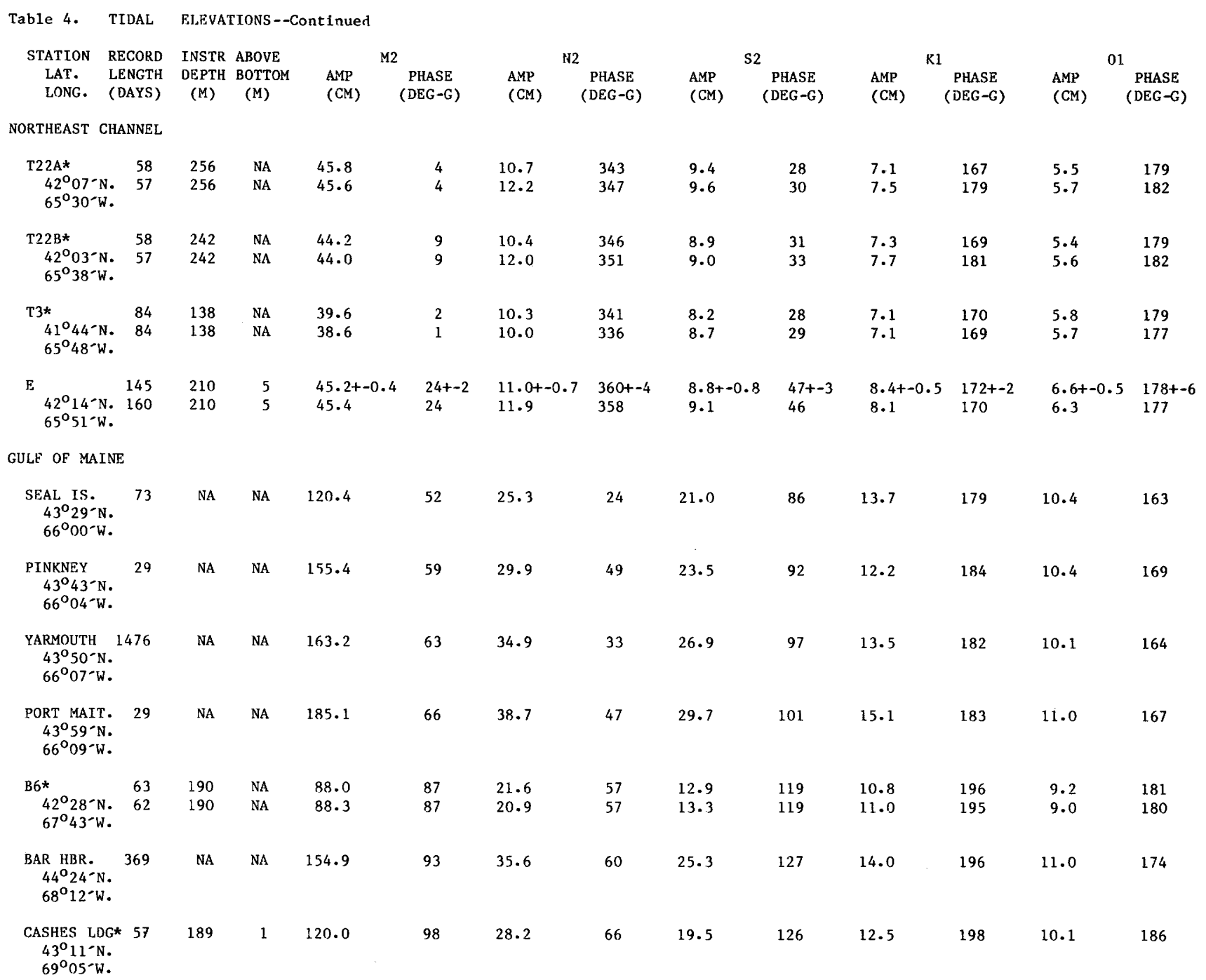

Instrument type, method of analysis and source of data are listed alphabetically by stations in Table 3.

* - Pressure data was converted to $\mathrm{cm}$ using $1 \mathrm{mb}=0.995 \mathrm{~cm}$.

NA - not available 
Table 4. Tidal constants for elevation-Continued

Table 4. TIDAL ELEVATIONS--Continued

STATION RECORD TNSTR ABOVE

LAT. LENGTH DEPTH BOTTOM

LONG. (DAYS)

AMP

M2

PHASE N2 PHASE

AMP

S2

(CM) (DEG-G)

AMP
(CM)

K1

GULF OF MAINE--CONT.

\begin{tabular}{|c|c|c|c|c|c|c|c|c|c|c|c|c|c|}
\hline $\begin{array}{l}\text { ROCKLAND } \\
44^{\circ} 06^{-} \mathrm{N} . \\
69^{\circ} 06^{-} \mathrm{W} .\end{array}$ & 29 & NA & NA & 150.0 & 98 & 33.0 & 65 & 19.5 & 133 & 15.2 & 201 & 11.8 & 170 \\
\hline $\begin{array}{l}\text { MONHEGAN* } \\
43^{\circ} 40^{\circ} \mathrm{N} . \\
69^{\circ} 23^{\circ} \mathrm{W} .\end{array}$ & 56 & 97 & 1 & $130 \cdot 3+-1 \cdot 2$ & $99+-1$ & 30.3 & 67 & 21.1 & 128 & $13.6+-0.9$ & $198+-4$ & 10.3 & 181 \\
\hline $\begin{array}{l}\text { NAUSET* } \\
41^{\circ} 49^{-} \mathrm{N} . \\
69^{\circ} 56^{\circ} \mathrm{W} .\end{array}$ & 58 & 7 & 1 & $103.2+-0.1$ & $102+-0$ & $22.2+-0.1$ & $70+-3$ & $14 \cdot 4+-1 \cdot 3$ & $133+-3$ & $13.1+-0.4$ & $201+-1$ & $11.5+-0.6$ & $182+-1$ \\
\hline $\begin{array}{l}\text { C.COD LT.** } \\
42^{\circ} 03^{\circ} \mathrm{N} . \\
70^{\circ} 05^{-} \mathrm{W} .\end{array}$ & * 29 & NA & NA & 116.0 & 113 & NA & NA & NA & NA & NA & NA & NA & NA \\
\hline $\begin{array}{l}\text { PORTLAND } \\
43^{\circ} 39^{-} \mathrm{N} . \\
70^{\circ} 15^{-} \mathrm{W} .\end{array}$ & 1845 & NA & NA & 133.0 & 103 & 29.6 & 73 & 21.7 & 138 & 13.9 & 202 & 11.1 & 183 \\
\hline $\begin{array}{l}\text { C.PORPOISE* } \\
43^{\circ} 13^{-} \mathrm{N} . \\
70^{\circ} 17^{-} \mathrm{W} .\end{array}$ & 73 & 97 & 1 & $127 \cdot 2+-1 \cdot 2$ & $103+-1$ & 29.9 & 71 & 20.3 & 134 & $12.9+-0.9$ & $204 t-4$ & 10.6 & 185 \\
\hline $\begin{array}{l}\text { C. COD CANL } \\
41^{\circ} 46^{-} \mathrm{N} . \\
70^{\circ} 30^{-} \mathrm{W} .\end{array}$ & 369 & NA & NA & 124.4 & 109 & 28.9 & 74 & 19.9 & 144 & 13.1 & 206 & 10.8 & 187 \\
\hline $\begin{array}{l}\text { PORTSMOUTH } \\
43^{\circ} 05^{-} \mathrm{N} . \\
70^{\circ} 44^{-} \mathrm{W} .\end{array}$ & 365 & NA & NA & 130.3 & 107 & 27.8 & 76 & 20.3 & 143 & 14.1 & 204 & 11.2 & 185 \\
\hline $\begin{array}{l}\text { BOSTON } 1 \\
42^{\circ} 21^{-} \mathrm{N} . \\
71^{\circ} 03^{-} \mathrm{W} .\end{array}$ & 1845 & NA & NA & 134.5 & 111 & 30.1 & 82 & 21.9 & 146 & 14.0 & 207 & 11.1 & 189 \\
\hline $\begin{array}{l}\text { ISLE HAUTE } \\
45^{\circ} 15^{-} \mathrm{N} \text {. } \\
65^{\circ} 01^{-} \text {-W. }\end{array}$ & 45 & NA & NA & 418.8 & 98 & 36.6 & 74 & 42.4 & 159 & 16.5 & 196 & 11.9 & 158 \\
\hline $\begin{array}{l}\text { MARGARETVIL } \\
45^{\circ} 03^{\circ} \mathrm{N} \text {. } \\
65^{\circ} 03^{-} \mathrm{W} .\end{array}$ & 58 & NA & NA & 387.4 & 92 & 114.9 & 63 & 42.4 & 146 & 15.5 & 188 & 13.7 & 171 \\
\hline
\end{tabular}


Table 4. Tidal constants for elevation-Continued

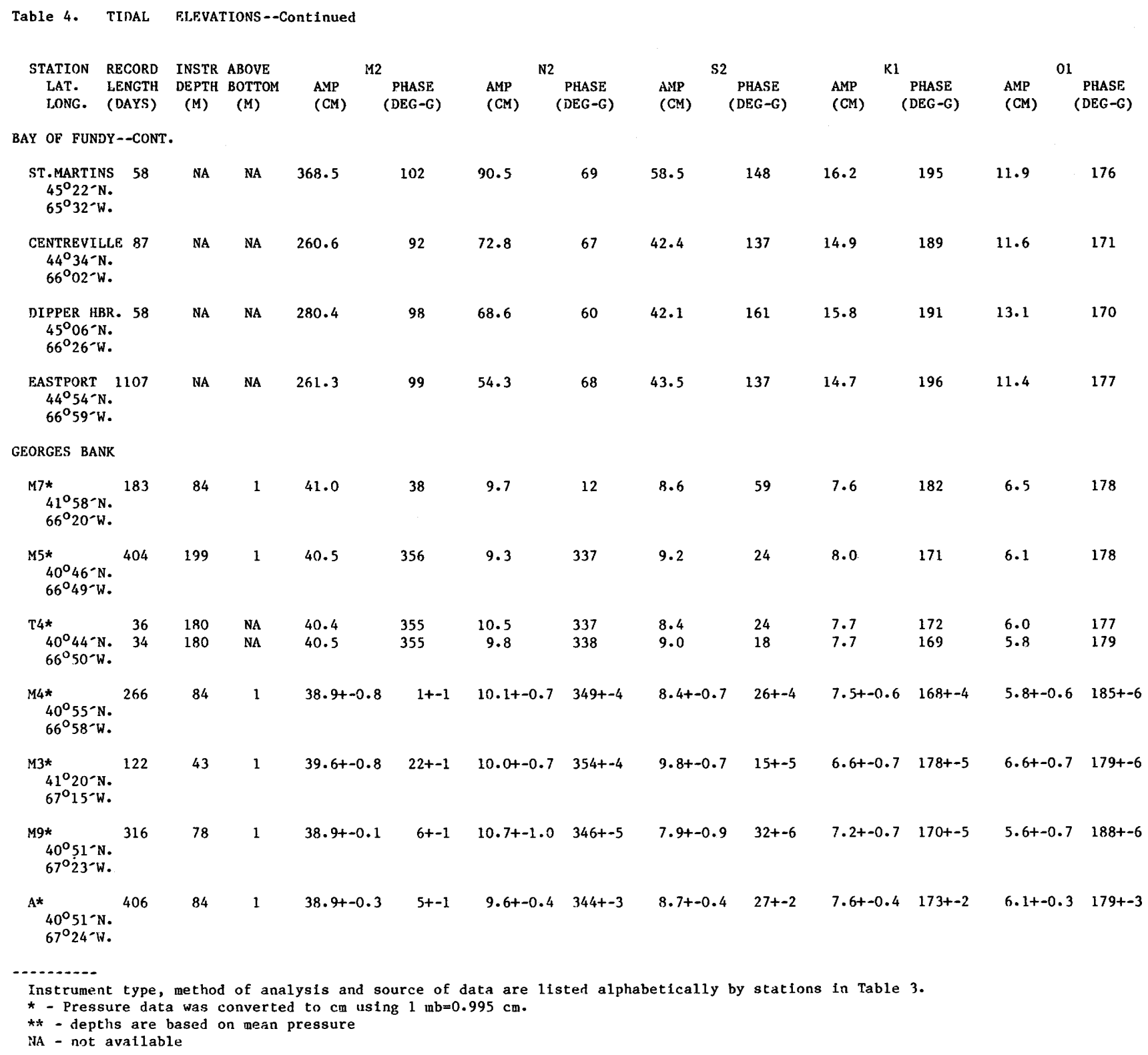


Table 4. Tidal constants for elevation-Continued

Table 4. TIDAL ELEVATIONS--Continued

\begin{tabular}{clllllllllllll} 
STATION & RECORD & INSTR ABOVE & \multicolumn{3}{c}{ M2 } & \multicolumn{3}{c}{ N2 } & \multicolumn{3}{c}{ S2 } & \multicolumn{3}{c}{ K1 } \\
LAT. & LENGTH & DEPTH BOTTOM & AMP & PHASE & AMP & PHASE & AMP & PHASE & AMP & PHASE & AMP & PHASE \\
LONG. & $($ DAYS $)$ & (M) & (M) & (CM) & (DEG-G) & (CM) & (DEG-G) & (CM) & (DEG-G) & (CM) & (DEG-G) & (CM) & (DEG-G)
\end{tabular}

GEORGES BANK--CONT.

\begin{tabular}{|c|c|c|c|c|c|c|c|c|c|c|c|c|c|}
\hline $\begin{array}{l}\mathrm{K}^{*} \\
41^{\circ} 04^{-\mathrm{N}} \\
67^{\circ} 34^{-} \mathrm{W}\end{array}$ & 348 & 61 & 1 & $39.9+-0.5$ & $18+-2$ & $9.9+-0.4$ & $354+-2$ & $8.6+-0.3$ & $38+-4$ & $7.4+-0.4$ & $176+-1$ & $6.2+-0.4$ & $180+-2$ \\
\hline $\begin{array}{l}\text { D } 41^{\circ} 59^{-} \mathrm{N} . \\
67^{\circ} 47^{-} \mathrm{W} .\end{array}$ & 29 & 83 & 1 & 75.8 & 93 & 18.6 & 63 & 11.1 & 120 & 10.2 & 198 & 8.5 & 184 \\
\hline $\begin{array}{l}\text { M2* } \\
41^{\circ} 59^{-} \mathrm{N} . \\
67^{\circ} 47^{-} \mathrm{W} .\end{array}$ & 94 & 83 & 1 & 77.2 & 92 & 18.3 & 65 & 19.0 & 162 & 10.7 & 197 & 8.8 & 186 \\
\hline $\begin{array}{l}\mathrm{M} 1^{\star} \\
\quad 42^{\circ} 04^{-N} . \\
67^{\circ} 52^{-} \mathrm{W} .\end{array}$ & 556 & 179 & 5 & 78.2 & 92 & 18.0 & 63 & 12.2 & 121 & 11.2 & 199 & 8.5 & 185 \\
\hline
\end{tabular}

LYDONIA CANYON

\begin{tabular}{|c|c|c|c|c|c|c|c|c|c|c|c|c|c|}
\hline $\begin{array}{l}\mathrm{LCL} L^{*} \\
40^{\circ} 32^{-} \mathrm{N} . \\
67^{\circ} 36^{-} \mathrm{W} .\end{array}$ & 87 & 124 & 1 & $39.4+-0.1$ & $354+-1$ & $9.1+-0.4$ & $330+-2$ & $9.5+-0.4$ & $19+-1$ & $3.0+-0.3$ & $171+-2$ & $6.1+-0.2$ & $180+-1$ \\
\hline $\begin{array}{l}\mathrm{LC} 0^{*} \\
40^{\circ} 27^{\circ} \mathrm{N} . \\
67^{\circ} 40^{-} \mathrm{W} .\end{array}$ & 87 & 549 & 1 & $39.2+-0.3$ & $357+-1$ & $9.3+-0.7$ & $333+-3$ & $9.1+-0.3$ & $21+-2$ & $8.4+-0.5$ & $173+-1$ & $6.5+-0.2$ & $179+-3$ \\
\hline $\begin{array}{l}\mathrm{LCA*} \\
40^{\circ} 34^{-} \mathrm{N} . \\
67^{\circ} 45^{-} \mathrm{W} .\end{array}$ & 58 & 99 & 1 & $39.2+-0.0$ & $358+-2$ & $9.8+-0.3$ & $338+-6$ & $8.8+-0.3$ & $21+-3$ & $7.8+-0.4$ & $169+-1$ & $6.3+-0.1$ & $178+-1$ \\
\hline $\begin{array}{l}\mathrm{T} 23^{*} \\
40^{\circ} 22^{-} \mathrm{N} . \\
67^{\circ} 45^{-} \mathrm{W} .\end{array}$ & $\begin{array}{l}58 \\
57\end{array}$ & $\begin{array}{l}173 \\
173\end{array}$ & $\begin{array}{l}1 \\
1\end{array}$ & $\begin{array}{l}40.7 \\
40.4\end{array}$ & $\begin{array}{l}356 \\
356\end{array}$ & $\begin{array}{l}9.9 \\
8.8\end{array}$ & $\begin{array}{l}336 \\
341\end{array}$ & $\begin{array}{l}8.6 \\
8.7\end{array}$ & $\begin{array}{l}20 \\
22\end{array}$ & $\begin{array}{l}7.9 \\
7.6\end{array}$ & $\begin{array}{l}172 \\
162\end{array}$ & $\begin{array}{l}6.1 \\
6.1\end{array}$ & $\begin{array}{l}180 \\
183\end{array}$ \\
\hline $\begin{array}{l}\mathrm{LCM} * \\
40^{\circ} 30^{-} \mathrm{N} . \\
67^{\circ} 49^{-} \mathrm{W} .\end{array}$ & 174 & 119 & 1 & $39.4+-0.1$ & $356+-1$ & $9.5+-0.5$ & $334+-4$ & $9.2+-0.3$ & $21+-2$ & $3.0+-0.4$ & $172+-2$ & $6.2+-0.2$ & $180+-2$ \\
\hline
\end{tabular}

GREAT SOUTH CHANNEL

\begin{tabular}{|c|c|c|c|c|c|c|c|c|c|c|c|c|c|}
\hline $\begin{array}{l}\mathrm{B*} \\
40^{\circ} 49^{-} \mathrm{N} . \\
69^{\circ} 00^{-} \mathrm{W} .\end{array}$ & $\begin{array}{l}116 \\
136\end{array}$ & $\begin{array}{l}77 \\
77\end{array}$ & $\begin{array}{l}1 \\
1\end{array}$ & $\begin{array}{l}25.9+-0.0 \\
25.9\end{array}$ & $\begin{array}{l}47+-2 \\
47\end{array}$ & $\begin{array}{l}7.5+-0.6 \\
7.1\end{array}$ & $\begin{array}{l}20+-1 \\
21\end{array}$ & $\begin{array}{l}5.0+-0.0 \\
4.8\end{array}$ & $\begin{array}{l}59+-3 \\
58\end{array}$ & $\begin{array}{l}7.3+-1.0 \\
7.0\end{array}$ & $\begin{array}{l}196+-1 \\
193\end{array}$ & $\begin{array}{l}7.5+-1.0 \\
7.4\end{array}$ & $\begin{array}{l}192+-1 \\
192\end{array}$ \\
\hline $\begin{array}{l}\mathrm{R}^{*} \\
40^{\circ} 30^{-\mathrm{N}} . \\
69^{\circ} 07^{-} \mathrm{W} .\end{array}$ & 16 & 79 & 1 & $31.4+-0.4$ & $3+-1$ & $7.9+-0.3$ & $344 t-4$ & $7.6+-0.2$ & $21+-1$ & $7.3+-0.2$ & $178+-5$ & $6.3+-0.2$ & $185+-3$ \\
\hline KIWI* & 78 & 510 & 1 & 41.4 & 349 & 11.0 & 334 & 8.1 & 15 & 8.7 & 176 & 6.7 & 180 \\
\hline
\end{tabular}
$69^{\circ} 25^{-\mathrm{W}}$

(1)

Instrument type, method of analysis and source of data are listed alphabetically by stations in Table 3. * - Pressure data was converted to $\mathrm{cm}$ using $1 \mathrm{mb}=0.995 \mathrm{~cm}$.

NA - not available 
Table 4. Tidal constants for elevation-Continued

\begin{tabular}{|c|c|c|c|c|c|c|c|c|c|c|c|c|c|}
\hline STATION R & RECORD & INSTR & ABOVE & \multicolumn{2}{|l|}{ M2 } & \multicolumn{2}{|l|}{ N2 } & \multicolumn{2}{|c|}{$\mathrm{S} 2$} & \multicolumn{2}{|c|}{ K1 } & \multicolumn{2}{|c|}{01} \\
\hline $\begin{array}{l}\text { LAT. } \\
\text { LONG. }\end{array}$ & $\begin{array}{l}\text { LENGTH } \\
\text { (DAYS) }\end{array}$ & $\begin{array}{l}\text { DEPTH } \\
(M)\end{array}$ & $\begin{array}{l}\text { BOTTOM } \\
\text { (M) }\end{array}$ & $\begin{array}{l}\text { AMP } \\
(\mathrm{CM})\end{array}$ & $\begin{array}{c}\text { PHASE } \\
(D E G-G)\end{array}$ & $\begin{array}{l}\text { AMP } \\
(C M)\end{array}$ & $\begin{array}{c}\text { PHASE } \\
(\text { DEG-G) }\end{array}$ & $\begin{array}{l}\text { AMP } \\
(\mathrm{CM})\end{array}$ & $\begin{array}{c}\text { PHASE } \\
(\mathrm{DEG}-\mathrm{G})\end{array}$ & $\begin{array}{l}\text { AMP } \\
(C M)\end{array}$ & $\begin{array}{c}\text { PHASE } \\
(D E G-G)\end{array}$ & $\begin{array}{l}\text { AMP } \\
(\mathrm{CM})\end{array}$ & $\begin{array}{l}\text { PHASE } \\
\text { (DEG-G) }\end{array}$ \\
\hline \multicolumn{14}{|c|}{ NANTUCKET SHOALS } \\
\hline $\begin{array}{l}\mathrm{S} \\
41^{\circ} 11^{-} \mathrm{N} . \\
70^{\circ} 12^{-\mathrm{W}} .\end{array}$ & . & 25 & 0 & 32.3 & 1 & 9.1 & 339 & 7.8 & 21 & 6.1 & 177 & 5.8 & 197 \\
\hline $\begin{array}{l}\text { NSFE } 1 * \\
40^{\circ} 41-\mathrm{N} . \\
70^{\circ} 08^{-} \mathrm{W} .\end{array}$ & 365 & 45 & 1 & 38.7 & 356 & 9.4 & 340 & 8.9 & 18 & 6.5 & 173 & 5.6 & 190 \\
\hline $\begin{array}{l}\text { Q* } \\
40^{\circ} 30^{-} \mathrm{N} \\
70^{\circ} 13^{-} \mathrm{W}\end{array}$ & 116 & 66 & 1 & $38.7+-0.2$ & $353+-1$ & $9.3+-0.3$ & $337+-3$ & $8.7+-0.1$ & $18+-3$ & $7.3+-0.4$ & $173+-2$ & $5.9+-0.1$ & $186+-4$ \\
\hline $\begin{array}{l}\text { NSFE2* } \\
40^{\circ} 29^{-} \mathrm{N} . \\
70^{\circ} 13^{-} \mathrm{W} .\end{array}$ & . & 65 & 1 & 40.4 & 354 & 9.6 & 338 & 8.7 & 17 & 7.3 & 173 & 5.9 & 188 \\
\hline $\begin{array}{l}\text { NSFE4* } \\
40^{\circ} 13^{-N} . \\
70^{\circ} 19^{-} \mathrm{W} .\end{array}$ & 374 & 104 & 1 & 42.0 & 353 & 9.7 & 336 & 9.2 & 18 & 8.1 & 177 & 6.5 & 185 \\
\hline $\begin{array}{l}\text { NSFE } 5 * \\
40^{\circ} 02-\mathrm{N} . \\
70^{\circ} 23^{-W}\end{array}$ & 365 & 197 & 1 & 41.9 & 351 & $10 \cdot 3$ & 335 & 9.1 & 17 & 8.6 & 175 & 6.5 & 183 \\
\hline NEW ENGLAND & SHELF & & & & & & & & & & & & \\
\hline $\begin{array}{l}\mathrm{P}^{*} \\
40^{\circ} 29^{-\mathrm{N}} \\
70^{\circ} 30^{\prime} \mathrm{W} .\end{array}$ & . & 70 & 1 & $41 \cdot 6+-0.4$ & $352+-0$ & $10 \cdot 3+-0.2$ & $3.37+-3$ & $9.5+-0.0$ & $15+-1$ & $3.3+-0.5$ & $177+-2$ & $5.9+-0.2$ & $185+-1$ \\
\hline $\begin{array}{l}\mathrm{BBA}^{*} \\
41^{\circ} 38^{-} \mathrm{N} \\
70^{\circ} 40^{-} \mathrm{W}\end{array}$ & . & 13 & 1 & 53.8 & 8 & 13.8 & 351 & 11.6 & 32 & 6.6 & 168 & 4.9 & 204 \\
\hline $\begin{array}{l}\text { MENEMSHA } \\
41^{\circ} 20^{\circ} \mathrm{N} . \\
70^{\circ} 46^{-} \mathrm{W} .\end{array}$ & . & 1 & 0 & $45.1+-0.6$ & $5+-3$ & $11 \cdot 9+-0.2$ & $356+-5$ & $10.0+-0.1$ & $24+-4$ & $5.4+-0.5$ & $176+-8$ & $6.0+-0.8$ & $195+-1$ \\
\hline $\begin{array}{l}\mathrm{MIT} / \mathrm{WHOI} 3 \\
40^{\circ} 18^{\circ} \mathrm{N} . \\
70^{\circ} 54^{-\mathrm{W}} .\end{array}$ & . & 112 & 1 & $42 \cdot 2+-0.5$ & $347+-1$ & $10 \cdot 5+-0.1$ & $327+-1$ & $10.1+-0.1$ & $13+-1$ & $3.3+-0.8$ & $173+-5$ & $6.8+-0.6$ & $178+-5$ \\
\hline $\begin{array}{l}\text { NES7 } 63^{*} \\
39^{\circ} 56^{-} \mathrm{N} . \\
71^{\circ} 03^{-} \mathrm{W} .\end{array}$ & 136 & 516 & 1 & $43 \cdot 3+-0.5$ & $349+-1$ & 10.4 & 332 & 8.9 & 17 & $8.7+-0.4$ & $178+-2$ & 6.9 & 181 \\
\hline $\begin{array}{l}\text { CXL } \\
\quad 41^{\circ} 09^{-N} . \\
71^{\circ} 03^{-W} .\end{array}$ & T. & 34 & 0 & 44.4 & 1 & $10 \cdot 3$ & 334 & 9.9 & 0 & 6.5 & 178 & 5.1 & 183 \\
\hline
\end{tabular}

Instrument type, method of analysis and source of data are listed alphabetically by stations in Table 3. * - Pressure data was converted to $\mathrm{cm}$ using $1 \mathrm{mb}=0.995 \mathrm{~cm}$.

INA - not available 
Table 4. Tidal constants for elevation-Continued

\begin{tabular}{|c|c|c|c|c|c|c|c|c|c|c|c|c|c|}
\hline STAT LON & ECORD & INS'TR & ABOVE & \multicolumn{2}{|l|}{ M2 } & \multicolumn{2}{|l|}{ N2 } & \multicolumn{2}{|l|}{ S2 } & \multicolumn{2}{|c|}{$\mathrm{K} 1$} & \multicolumn{2}{|c|}{01} \\
\hline $\begin{array}{ll}\text { LAT. } & \text { LEI } \\
\text { LONG. } & \text { (D }\end{array}$ & $\begin{array}{l}\text { ENGTH } \\
\text { DAYS) }\end{array}$ & $\begin{array}{l}\text { DEPTH } \\
(\mathrm{M})\end{array}$ & $\begin{array}{l}\text { BOTTOM } \\
\text { (M) }\end{array}$ & $\begin{array}{l}\text { AMP } \\
\text { (CM) }\end{array}$ & $\begin{array}{c}\text { PHASE } \\
(D E G-G)\end{array}$ & $\begin{array}{l}\text { AMP } \\
(\mathrm{CM})\end{array}$ & $\begin{array}{l}\text { PHASE } \\
(D E G-G)\end{array}$ & $\begin{array}{l}\text { AMP } \\
\text { (CM) }\end{array}$ & $\begin{array}{l}\text { PHASE } \\
(D E G-G)\end{array}$ & $\begin{array}{l}\text { AMP } \\
(\mathrm{CM})\end{array}$ & $\begin{array}{l}\text { PHASE } \\
(D E G-G)\end{array}$ & $\begin{array}{l}\text { AMP } \\
(\mathrm{CM})\end{array}$ & $\begin{array}{c}\text { PHASE } \\
\text { (DEG-G) }\end{array}$ \\
\hline NEW ENGLAND SH & SHELF - & -CONT. & & & & & & & & & & & \\
\hline $\begin{array}{l}\text { PICKET* } \\
40^{\circ} 43^{-} \mathrm{N} \\
71^{\circ} 19^{-} \mathrm{W}\end{array}$ & 79 & 68 & 1 & $44.0+-0.8$ & $349+-1$ & 12.0 & 317 & 9.5 & 0 & $7.9+-0.5$ & $167+-4$ & 5.3 & 182 \\
\hline $\begin{array}{l}\text { NEWPORT } \\
41^{\circ} 30^{-} \mathrm{N} \\
71^{\circ} 20^{-} \mathrm{W}\end{array}$ & 1107 & NA & NA & $51 \cdot 3$ & 1 & 12.4 & 345 & 11.9 & 23 & 6.2 & 168 & 4.9 & 199 \\
\hline MIDJLLE ATLANT & TIC BLC & GHT & & & & & & & & & & & \\
\hline $\begin{array}{l}\mathrm{P} 32 \\
40^{\circ} 15^{-} \mathrm{N} . \\
71^{\circ} 52^{-} \mathrm{W} .\end{array}$ & 87 & 74 & 1 & 47.6 & 348 & 11.7 & 332 & 10.3 & 16 & 11.7 & 163 & 4.9 & 188 \\
\hline $\begin{array}{l}\text { MONTAUK } \\
41^{\circ} 05^{-} \mathrm{N} . \\
71^{\circ} 49^{-} \mathrm{W} .\end{array}$ & 113 & 19 & 0 & 33.5 & 11 & $8 \cdot 3$ & 358 & 7.8 & 35 & 7.2 & 164 & 4.1 & 192 \\
\hline $\begin{array}{l}\text { LT5* } \\
\quad 40^{\circ} 12^{-} \mathrm{N} . \\
72^{\circ} 00^{-} \mathrm{W} .\end{array}$ & 183 & 67 & 1 & $46.8+-0.6$ & $349+-1$ & 11.2 & 331 & 10.5 & 15 & $8.3+-0.3$ & $175+-2$ & 6.0 & 184 \\
\hline $\begin{array}{l}\text { MESA } \\
\qquad \begin{array}{l}39^{\circ} 13^{-} \mathrm{N} \\
72^{\circ} 10^{-} \mathrm{W}\end{array}\end{array}$ & 29 & 840 & 1 & 43.6 & 345 & 9.1 & 332 & 9.5 & 8 & 8.5 & 170 & 8.1 & 185 \\
\hline $\begin{array}{l}\mathrm{P} 31 \\
40^{\circ} 39^{-} \mathrm{N} . \\
72^{\circ} 15^{-} \mathrm{W}\end{array}$ & 87 & 46 & 1 & 49.6 & 346 & 12.0 & 330 & 10.9 & 15 & 10.4 & 158 & 4.0 & 185 \\
\hline $\begin{array}{l}\text { LT4* } \\
40^{\circ} 34-\mathrm{N} . \\
72^{\circ} 19^{-} \mathrm{W} .\end{array}$ & 61 & 50 & 1 & $48 \cdot 2+-1.0$ & $347+-1$ & 11.9 & 330 & 11.5 & 12 & $8.0+-0.8$ & $166+-5$ & 5.6 & 180 \\
\hline $\begin{array}{c}\text { SHINNECOCK } \\
40^{\circ} 50^{-} \mathrm{N} . \\
72^{\circ} 29^{-} \mathrm{W} .\end{array}$ & 29 & NA & NA & 46.7 & 345 & $11 \cdot 5$ & 329 & 9.7 & 7 & 5.7 & 166 & 2.8 & 201 \\
\hline $\begin{array}{l}\text { ME* } \\
\begin{array}{l}39^{\circ} 57^{\circ} \mathrm{N} . \\
72^{\circ} 36^{-} \mathrm{W}\end{array}\end{array}$ & 29 & 58 & 1 & $48.3+-0.1$ & $347+-0$ & $10.6+-0.5$ & $332+-3$ & $11.0+-0.4$ & $14+-0$ & $9.0+-0.3$ & $172+-3$ & $5.9+-0.8$ & $175+-2$ \\
\hline $\begin{array}{l}\mathrm{P} 22 \mathrm{C} \\
39^{\circ} 39^{-} \mathrm{N} . \\
72^{\circ} 38^{-} \mathrm{W} .\end{array}$ & 30 & 77 & 1 & 47.8 & 349 & 11.6 & 333 & 10.4 & 13 & 8.5 & 181 & 6.7 & 179 \\
\hline
\end{tabular}

Instrument type, method of analysis and source of data are listed alphabetically by stations in Table 3 . * - Pressure data was converted to $\mathrm{cm}$ using $1 \mathrm{mb}=0.995 \mathrm{~cm}$.

NA - not available 
Table 4. Tidal constants for elevation-Continued

Table 4. TIDAL FILEVATIONS--Continued

\begin{tabular}{|c|c|c|c|c|c|c|c|c|c|c|c|c|c|}
\hline STATION & RECORD & INSTR & ABOVE & \multicolumn{2}{|c|}{$M 2$} & \multicolumn{2}{|c|}{ N2 } & \multicolumn{2}{|c|}{ S2 } & \multicolumn{2}{|c|}{$\mathrm{Kl}$} & \multicolumn{2}{|c|}{01} \\
\hline $\begin{array}{l}\text { LAT. } \\
\text { LONG. }\end{array}$ & $\begin{array}{l}\text { LENGTH } \\
\text { (DAYS) }\end{array}$ & $\begin{array}{c}\text { DEPTH } \\
\text { (M) }\end{array}$ & $\begin{array}{l}\text { BOTTOM } \\
\text { (M) }\end{array}$ & $\begin{array}{l}\text { AMP } \\
\text { (CM) }\end{array}$ & $\begin{array}{c}\text { PHASE } \\
(\text { DEG -G) }\end{array}$ & $\begin{array}{l}\text { AMP } \\
\text { (CM) }\end{array}$ & $\begin{array}{c}\text { PHASE } \\
(\text { DEG-G) }\end{array}$ & $\begin{array}{l}\text { AMP } \\
\text { (CM) }\end{array}$ & $\begin{array}{c}\text { PHASE } \\
\text { (DEG-G) }\end{array}$ & $\begin{array}{l}\text { AMP } \\
\text { (CM) }\end{array}$ & $\begin{array}{c}\text { PHASE } \\
(D E G-G)\end{array}$ & $\begin{array}{l}\text { AMP } \\
(\mathrm{CM})\end{array}$ & PHASE \\
\hline
\end{tabular}

MIDDLE ATLANTIC BIGHT - - CONT.

\begin{tabular}{|c|c|c|c|c|c|c|c|c|c|c|c|c|c|}
\hline $\begin{array}{l}\text { MESA9 } \\
\begin{array}{l}39^{\circ} 46^{-} \mathrm{N} . \\
72^{\circ} 42^{-} \mathrm{W} .\end{array}\end{array}$ & 22 & 56 & 1 & $49.8+-0.6$ & $351+-1$ & $11 \cdot 3+-0.1$ & $330+-1$ & $10.6+-0.1$ & $17+-1$ & $9.5+-0.4$ & $174+-2$ & $6.6+-0.2$ & $181+-2$ \\
\hline $\begin{array}{l}\text { LTM } \\
\qquad \begin{array}{l}40^{\circ} 07^{-\mathrm{N}} \\
72^{\circ} 55^{-} \mathrm{W}\end{array}\end{array}$ & 184 & 47 & 1 & 53.4 & 348 & 12.8 & 331 & 11.8 & 14 & 9.2 & 169 & 5.9 & 174 \\
\hline $\begin{array}{l}\text { MA* } \\
39^{\circ} 27^{-} \mathrm{N} . \\
73^{\circ} 00^{-} \mathrm{W} .\end{array}$ & 58 & 58 & 1 & 48.1 & 348 & 10.9 & 333 & 10.9 & 13 & 8.9 & 175 & 6.8 & 179 \\
\hline $\begin{array}{l}\text { MESA10* } \\
40^{\circ} 00^{-} \mathrm{N} . \\
73^{\circ} 14^{-} \mathrm{W}\end{array}$ & 22 & 45 & 1 & $55.5+-2.2$ & $351+-2$ & $12.8+-0.5$ & $332+-2$ & $12.5+-0.5$ & $16+-2$ & $3.8+-1.8$ & $172+-10$ & $6.5+-1.2$ & $170+-10$ \\
\hline $\begin{array}{l}\text { MC* } \\
38^{\circ} 33^{-N} . \\
73^{\circ} 31-W .\end{array}$ & 116 & 79 & 1 & $44.2+-0.5$ & $351+-1$ & $10.3+-0.4$ & $334+-2$ & $9.6+-0.5$ & $17+-1$ & $8.9+-0.4$ & $179+-1$ & $6.9+-0.3$ & $181+-2$ \\
\hline $\begin{array}{l}\text { MESAl } 1 * \\
40^{\circ} 08^{-} \mathrm{N} . \\
73^{\circ} 34^{-} \mathrm{W} .\end{array}$ & 22 & 36 & 1 & $58.9+-3.0$ & $350+-3$ & $13.8+-0.7$ & $330+-3$ & $13.1+-0.7$ & $15+-3$ & $10.0+-2.3$ & $167+-13$ & $6.3+-1.5$ & $169+-13$ \\
\hline $\begin{array}{l}\mathrm{MB}^{*} \\
38^{\circ} 44-\mathrm{N} . \\
73^{\circ} 38-\mathrm{W}\end{array}$ & 406 & 59 & 1 & $46.7+-0.3$ & $350+-1$ & $10.7+-0.6$ & $332+-3$ & $3.7+-0.5$ & $16+-2$ & $9.3+-0.3$ & $179+-3$ & $6.9+-0.3$ & $180+-2$ \\
\hline $\begin{array}{l}\text { EPA1 } \\
\qquad \begin{array}{l}39^{\circ} 38^{-N} \\
73^{\circ} 42^{-} \mathrm{W}\end{array}\end{array}$ & 29 & NA & NA & 54.8 & 347 & 12.4 & 328 & 10.8 & 18 & 10.3 & 169 & 7.2 & 167 \\
\hline $\begin{array}{l}\text { LT } 2 * \\
39^{\circ} 24^{-\mathrm{N}} . \\
73^{\circ} 44^{-\mathrm{W}} .\end{array}$ & 180 & 32 & 1 & 54.6 & 353 & 13.0 & 335 & 11.8 & 19 & 9.7 & 177 & 6.8 & 172 \\
\hline $\begin{array}{l}\text { AMBROSE } \\
40^{\circ} 28^{-} \mathrm{N} . \\
73^{\circ} 50^{-} \mathrm{W} .\end{array}$ & 58 & NA & $\mathrm{NA}$ & 65.0 & 353 & 15.6 & 336 & 13.5 & 18 & 10.3 & 176 & 6.3 & 158 \\
\hline $\begin{array}{l}\text { BATTERY } \\
40^{\circ} 42^{-N} . \\
74^{\circ} 01-\mathrm{W} .\end{array}$ & 1476 & NA & $\mathrm{NA}$ & 64.1 & 23 & 14.3 & 4 & 13.3 & 48 & 9.8 & 182 & 5.2 & 181 \\
\hline $\begin{array}{r}\text { SANDYHOOK } \\
40^{\circ} 28^{-} \mathrm{N} . \\
74^{\circ} 01^{-} \mathrm{W} .\end{array}$ & 1107 & NA & $\mathrm{NA}$ & $65.6+-3.5$ & $7+-3$ & $14 \cdot 4+-0.8$ & $352+-3$ & $13.7+-0.7$ & $34+-3$ & $3.7+-2.8$ & $176+-17$ & $5.2+-1.5$ & $172+-17$ \\
\hline
\end{tabular}


Table 4. Tidal constants for elevation-Continued

Table 4. TIDAL ELEVATIONS--Continued

\begin{tabular}{clllllllllllll} 
STATION & RECORD & INSTR ABOVE & \multicolumn{3}{c}{ M2 } & \multicolumn{3}{c}{ N2 } & \multicolumn{3}{c}{ S2 } & \multicolumn{3}{c}{ K1 } \\
LAT. & LENGTH & DEPTH & BOTTOM & AMP & PHASE & AMP & PHASE & AMP & PHASE & AMP & PHASE & AMP & PHASE \\
LONG. & (DAYS) & (M) & (M) & (CM) & (DEG-G) & (CM) & (DEG-G) & (CM) & (DEG-G) & (CM) & (DEG-G) & (CM) & (DEG-G)
\end{tabular}

MIDDLE ATLANTIC BIGHT

\begin{tabular}{|c|c|c|c|c|c|c|c|c|c|c|c|c|c|}
\hline $\begin{array}{l}\mathrm{MD}^{*} \\
38^{\circ} 59^{-} \mathrm{N} . \\
74^{\circ} \mathrm{O} 2^{-} \mathrm{W} .\end{array}$ & 116 & 40 & 1 & $52.4+-0.3$ & $354+-1$ & $12.0+-0.7$ & $335+-4$ & $11.1+-0.3$ & $17+-1$ & $10.2+-0.5$ & $182+-2$ & $7.5+-0.6$ & $175+-5$ \\
\hline $\begin{array}{l}\text { L. EGG } \\
39^{\circ} 28^{-} \mathrm{N} . \\
74^{\circ} 16^{-} \mathrm{W} .\end{array}$ & 203 & NA & NA & 59.1 & 358 & 14.9 & 338 & 11.8 & 25 & 12.0 & 176 & 7.4 & 163 \\
\hline $\begin{array}{l}\text { ATL.CITY } \\
39^{\circ} 21^{\circ} \mathrm{N} . \\
74^{\circ} 25^{-\mathrm{W}} .\end{array}$ & 369 & $\mathrm{NA}$ & NA & 58.3 & 354 & 13.8 & 335 & 12.3 & 18 & 10.9 & 180 & 7.3 & 167 \\
\hline $\begin{array}{l}\text { CAPE MAY } \\
38^{\circ} 58^{\circ} \mathrm{N} . \\
74^{\circ} 58^{-} \mathrm{W} .\end{array}$ & 365 & NA & $\mathrm{NA}$ & 70.4 & 30 & 16.0 & 14 & 12.7 & 56 & 10.4 & 200 & 8.4 & 188 \\
\hline
\end{tabular}

SOUTHERN MID-ATLANTIC BIGHT

\begin{tabular}{|c|c|c|c|c|c|c|c|c|c|c|c|c|c|}
\hline $\begin{array}{c}\text { BREAKWTR. } 1 \\
38^{\circ} 47^{\circ} \mathrm{N} . \\
75^{\circ} 06^{\circ} \mathrm{W} .\end{array}$ & 1107 & $\mathrm{NA}$ & $\mathrm{NA}$ & 59.1 & 30 & 13.0 & 8 & 11.2 & 53 & 10.3 & 201 & 8.4 & 188 \\
\hline $\begin{array}{l}\text { WALLLOPS IS } \\
37^{\circ} 50^{-} \mathrm{N} \text {. } \\
75^{\circ} 28^{-} \mathrm{W} .\end{array}$ & 29 & $\mathrm{NA}$ & $\mathrm{NA}$ & 50.7 & 355 & 12.3 & 334 & 11.1 & 17 & 7.2 & 170 & 7.3 & 198 \\
\hline $\begin{array}{l}\text { OCEAN CITY } \\
38^{\circ} 19^{-} \mathrm{N} \text {. } \\
75^{\circ} 05^{-} \mathrm{W} .\end{array}$ & 145 & NA & $\mathrm{NA}$ & 49.0 & 3 & 12.2 & 346 & 9.0 & 30 & 7.8 & 193 & 8.4 & 187 \\
\hline $\begin{array}{l}\text { C. HATTERAS } \\
35^{\circ} 13^{-} \mathrm{N} . \\
75^{\circ} 38^{-} \mathrm{W} .\end{array}$ & 29 & NA & $\mathrm{NA}$ & 43.9 & 356 & 9.8 & 340 & 5.3 & 11 & 9.2 & 186 & 6.5 & 184 \\
\hline \multicolumn{14}{|l|}{ CEANIC } \\
\hline $\begin{array}{l}\text { REIKO-MAY } \\
27^{\circ} 58^{-} \mathrm{N} . \\
69^{\circ} 40^{-} \mathrm{W} .\end{array}$ & 35 & NA & 0 & 34.6 & 1 & 8.2 & 339 & 6.8 & 33 & 7.8 & 195 & 6.2 & 196 \\
\hline $\begin{array}{l}\text { NOAA } \\
\quad 37^{\circ} 22^{\circ} \mathrm{N} . \\
73^{\circ} 05^{\circ} \mathrm{W} .\end{array}$ & 29 & 2743 & NA & 43.2 & 340 & 9.8 & 323 & 8.8 & 6 & 8.5 & 171 & 7.4 & 178 \\
\hline $\begin{array}{l}\text { GOBI -IV } \\
38^{\circ} 10^{-} \mathrm{N} . \\
71^{\circ} 22^{-} \mathrm{W} .\end{array}$ & 43 & 2516 & NA & 44.5 & 350 & 11.1 & 341 & 9.1 & 26 & 6.3 & 181 & 9.6 & 166 \\
\hline
\end{tabular}

Instrument type, method of analysis and source of data are listed alphabetically by stations in Table 3.

* - Pressure data was converted to $\mathrm{cm}$ using $1 \mathrm{mb}=0.995 \mathrm{~cm}$.

NA - not available 
Table 5. $M_{2}$ tidal current parameters. The stations are grouped by region (see plate 1) and are listed within each region from east to west. For each station, the latitude and longitude, the record length (days), the instrument depth $(\mathrm{m}$ ), the amplitude (in $\mathrm{cm} / \mathrm{s}$ ) and the phase (in ${ }^{\circ} \mathrm{G}$ ) for the east and north current, and the four ellipse parameters (UMAJOR, UMINOR, PHASE, and ORIENTATION) are tabulated. The $( \pm)$ number which follows some constants is the standard deviation of the estimates computed by the harmonic method for each data piece, or the standard error where the constants have been determined by the response method (see Data analysis for discussion).

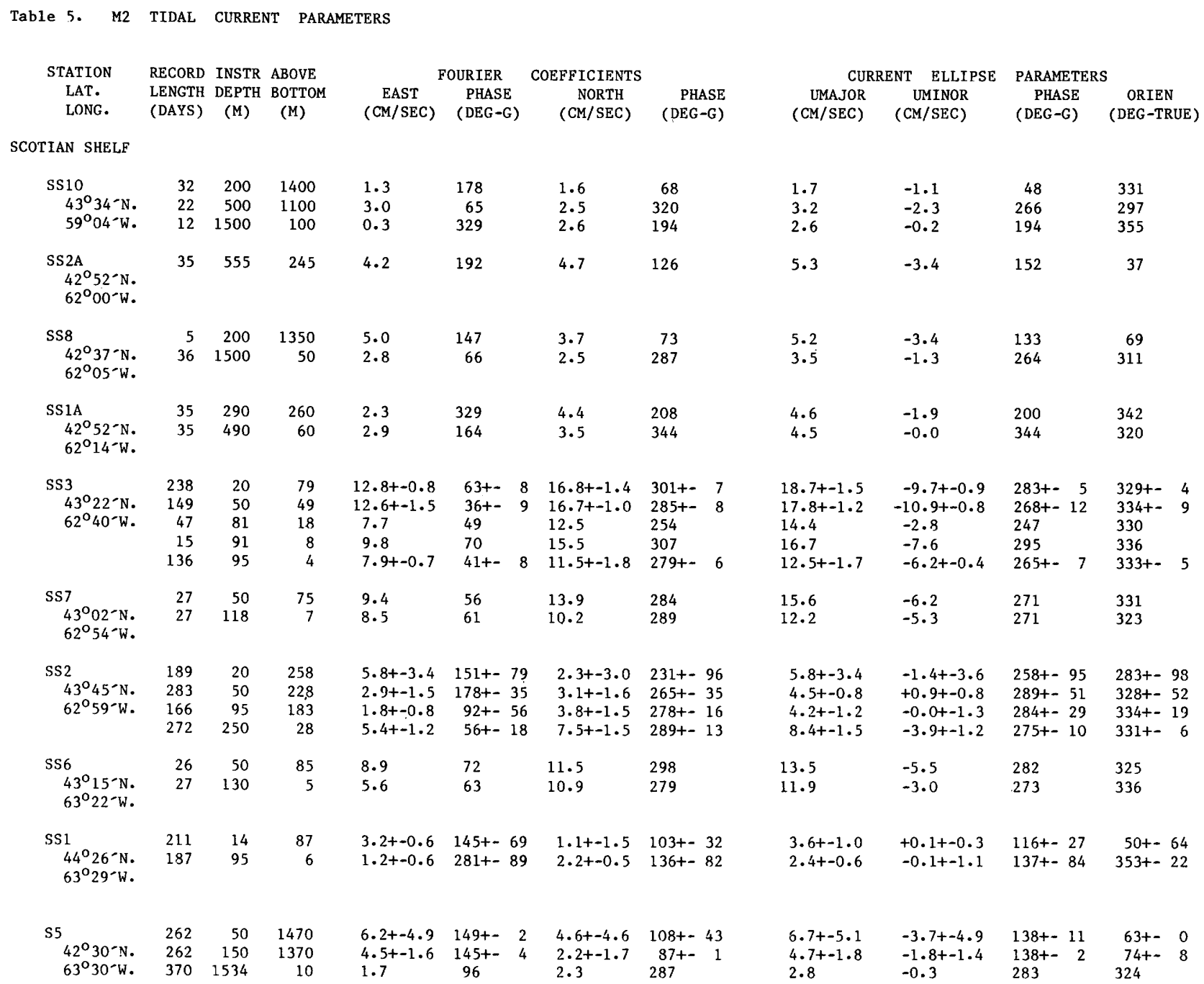

Instrument type, method of analysis and source of data are listed alphabetically by stations in Table 3 . If UMINOR $<0$, ellipse rotates clockwise 
Table 5. $\mathrm{M}_{2}$ tidal current parameters-Continued

Table 5. M2 TIDAL CURRENT PARAMETERS--Continued.

\begin{tabular}{|c|c|c|c|c|c|c|c|c|c|c|c|}
\hline TATION & RECORD & INSTR & ABOVE & & OURIER & COEFFICIENTS & & & ELLIPSE & PARAMETER & \\
\hline $\begin{array}{l}\text { LAT. } \\
\text { LONG. }\end{array}$ & $\begin{array}{l}\text { LENGTH } \\
\text { (DAYS) }\end{array}$ & $\begin{array}{l}\text { DEPTH } \\
\text { (M) }\end{array}$ & $\begin{array}{l}\text { BOTTOM } \\
\text { (M) }\end{array}$ & $\begin{array}{c}\text { EAST } \\
(\mathrm{CM} / \mathrm{SEC})\end{array}$ & $\begin{array}{c}\text { PHASE } \\
(\text { DEG -G) }\end{array}$ & $\begin{array}{c}\text { NORTH } \\
(\mathrm{CM} / \mathrm{SEC})\end{array}$ & $\begin{array}{r}\text { PHASE } \\
(\text { DEG-G) }\end{array}$ & $\begin{array}{r}\text { UMAJOR } \\
\text { (CM/SEC) }\end{array}$ & $\begin{array}{l}\text { UMINOR } \\
(\mathrm{CM} / \mathrm{SEC})\end{array}$ & $\begin{array}{r}\text { PHASE } \\
(\mathrm{DEG}-\mathrm{G})\end{array}$ & $\begin{array}{c}\text { ORIEN } \\
\text { (DEG-TRUE) }\end{array}$ \\
\hline
\end{tabular}

SCOTIAN SHELF - -CONT.

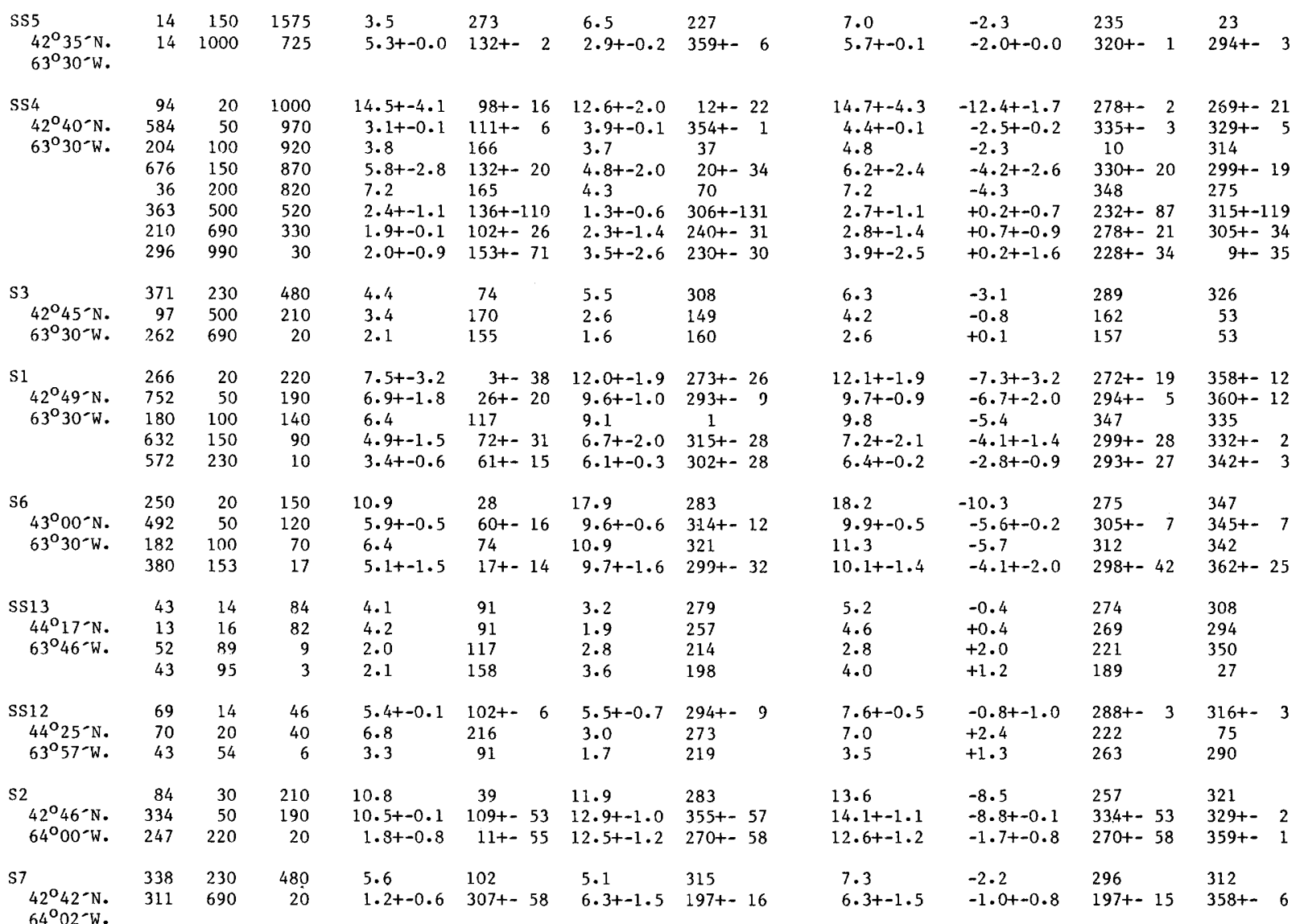

Instrument type, method of analysis and source of data are listed alphabetically by stations in Table 3.

If UMINOR $<0$, ellipse rotates clockwise 
Table 5. $M_{2}$ tidal current parameters-Continued

Table 5. M2 TIDAL CURRERT PARAMETERS-Continued

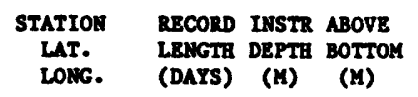

Long.

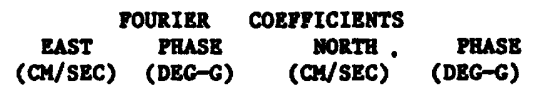

\begin{tabular}{|c|c|c|c|}
\hline CUE & BNT ELLIPSE & RARAYRTERS & \\
\hline $\begin{array}{c}\text { Dinwor } \\
\text { cy/sEC) }\end{array}$ & $\begin{array}{r}\text { DAINOR } \\
\text { (CY/SEC) }\end{array}$ & $\begin{array}{c}\text { PHASE } \\
\text { (DEC-G) }\end{array}$ & $\begin{array}{c}\text { ORIEH } \\
\text { (DRG-TRUB }\end{array}$ \\
\hline
\end{tabular}

SCOTIAY SEELP-CONT.

C5

\begin{tabular}{|c|c|c|c|c|c|c|c|c|}
\hline $\begin{array}{l}43^{\circ} 34^{\prime} \mathrm{H} . \\
65^{\circ} 06^{\prime} \mathrm{H} .\end{array}$ & $\begin{array}{l}170 \\
174 \\
173\end{array}$ & $\begin{array}{l}15 \\
30 \\
50\end{array}$ & $\begin{array}{l}45 \\
30 \\
10\end{array}$ & $\begin{array}{l}4.6+-3.3 \\
13.6 \\
10.1\end{array}$ & $\begin{array}{l}90+- \\
333 \\
198\end{array}$ & 41 & $\begin{array}{l}7.2+-4.0 \\
5.0 \\
6.3\end{array}$ & $\begin{array}{l}293+ \\
142 \\
293\end{array}$ \\
\hline $\begin{array}{l}43^{\circ} 11^{\prime} \mathrm{K} . \\
65^{\circ} 43^{\prime} \mathrm{H} .\end{array}$ & $\begin{array}{l}162 \\
162 \\
174\end{array}$ & $\begin{array}{l}15 \\
30 \\
50\end{array}$ & $\begin{array}{l}45 \\
30 \\
10\end{array}$ & $\begin{array}{l}85.1+2.3 \\
75.1 t-2.0 \\
41.6 t-10.4\end{array}$ & $\begin{array}{l}158+ \\
141+ \\
150+\end{array}$ & $\begin{array}{r}2 \\
2 \\
14\end{array}$ & $\begin{array}{l}21.2+-3.7 \\
22.8+-4.0 \\
16.6+-3.2\end{array}$ & $\begin{array}{l}12+ \\
333+ \\
308+\end{array}$ \\
\hline $\begin{array}{l}42^{\circ} 50^{\prime K} . \\
65^{\circ} 50^{\circ} \mathrm{W} .\end{array}$ & $\begin{array}{l}161 \\
103 \\
161\end{array}$ & $\begin{array}{r}15 \\
50 \\
100\end{array}$ & $\begin{array}{l}95 \\
60 \\
10\end{array}$ & $\begin{array}{l}45.6+-1.6 \\
53.5+1.4 \\
35.6+-1.8\end{array}$ & $\begin{array}{l}174 t \\
162+ \\
162+\end{array}$ & $\begin{array}{l}2 \\
2 \\
3\end{array}$ & $\begin{array}{l}29.5+-3.6 \\
35.2+-1.8 \\
27.7+2.5\end{array}$ & $\begin{array}{l}16+ \\
11+ \\
359+\end{array}$ \\
\hline
\end{tabular}

NORTHEAST CHANREL

\begin{tabular}{|c|c|c|c|c|c|c|c|c|}
\hline $\begin{array}{l}\mathrm{NECl} \\
42^{\circ} 22^{\prime} \mathrm{N} . \\
65^{\circ} 56^{\prime} \mathrm{W} .\end{array}$ & $\begin{array}{l}174 \\
174 \\
174\end{array}$ & $\begin{array}{l}103 \\
153 \\
207\end{array}$ & $\begin{array}{r}120 \\
70 \\
16\end{array}$ & $\begin{array}{l}42.3+-4.1 \\
45.1+-2.6 \\
27.6+-3.2\end{array}$ & $\begin{array}{l}162+- \\
157+ \\
141+\end{array}$ & $\begin{array}{l}5 \\
3 \\
4\end{array}$ & $\begin{array}{l}33.3+-1.5 \\
41.6+-3.1 \\
40.6+-1.9\end{array}$ & $\begin{array}{l}18+ \\
18+ \\
350+\end{array}$ \\
\hline $\begin{array}{l}\mathrm{NEC} 2^{*} \\
42^{\circ} 18^{\circ} \mathrm{H} . \\
65^{\circ} 58^{\circ} \mathrm{H} .\end{array}$ & $\begin{array}{r}58 \\
174 \\
58\end{array}$ & $\begin{array}{l}106 \\
156 \\
217\end{array}$ & $\begin{array}{r}134 \\
84 \\
17\end{array}$ & $\begin{array}{l}38.3+-1.0 \\
45.3+-3.5 \\
31.9+-9.3\end{array}$ & $\begin{array}{l}166+- \\
153+ \\
147+\end{array}$ & $\begin{array}{l}2 \\
2 \\
7\end{array}$ & $\begin{array}{l}32.0+0.6 \\
41.7+2.9 \\
30.6+8.2\end{array}$ & $\begin{array}{l}14 t \\
19+ \\
9+\end{array}$ \\
\hline $\begin{array}{l}\operatorname{NEC} 3^{*} \\
42^{\circ} 11^{\prime} \mathrm{N} . \\
66^{\circ} 02^{\prime} \mathrm{W} .\end{array}$ & $\begin{array}{r}87 \\
87 \\
174\end{array}$ & $\begin{array}{l}112 \\
162 \\
220\end{array}$ & $\begin{array}{r}116 \\
66 \\
16\end{array}$ & $\begin{array}{l}46.7 t-2.8 \\
43.6+-3.3 \\
27.4 t-3.2\end{array}$ & $\begin{array}{l}170 t \\
154 t \\
142+\end{array}$ & $\begin{array}{l}1 \\
4 \\
7\end{array}$ & $\begin{array}{l}37.7+-2.4 \\
50.6+-1.2 \\
43.5+-3.9\end{array}$ & $\begin{array}{l}21+ \\
22+ \\
357+\end{array}$ \\
\hline
\end{tabular}

GULF OF MAINE

\begin{tabular}{|c|c|c|c|c|c|c|c|c|c|c|c|c|c|}
\hline $\begin{array}{l}\text { CASHES LEDGE } \\
43^{\circ} 11^{\prime} \mathrm{N} . \\
69^{\circ} 05^{\prime} \mathrm{W} .\end{array}$ & $\begin{array}{l}58 \\
58 \\
58\end{array}$ & $\begin{array}{r}33 \\
68 \\
118\end{array}$ & $\begin{array}{r}157 \\
122 \\
72\end{array}$ & $\begin{array}{l}8.2 \\
5.7+0.7 \\
5.8+-0.7\end{array}$ & $\begin{array}{l}233 \\
240+- \\
218+-\end{array}$ & $\begin{array}{l}7 \\
7\end{array}$ & $\begin{array}{l}11.9 \\
7.1+-1.2 \\
8.6+-0.7\end{array}$ & $\begin{array}{c}10 \\
16+ \\
346+\end{array}$ & $\begin{array}{r}10 \\
5\end{array}$ & $\begin{array}{r}13.6 \\
8.5 \\
9.5\end{array}$ & $\begin{array}{l}+4.9 \\
+3.3 \\
+4.1\end{array}$ & $\begin{array}{r}22 \\
32 \\
359\end{array}$ & $\begin{array}{l}329 \\
324 \\
332\end{array}$ \\
\hline $\begin{array}{l}\text { MONHEGAN } \\
43^{\circ} 40^{\prime} \mathrm{H} \\
69^{\circ} 23^{\circ} \mathrm{H}\end{array}$ & $\begin{array}{l}57 \\
58\end{array}$ & $\begin{array}{l}33 \\
68\end{array}$ & $\begin{array}{l}65 \\
30\end{array}$ & $\begin{array}{l}4.2 \\
3.4+1.3\end{array}$ & $\begin{array}{l}287 \\
338+-\end{array}$ & 22 & $\begin{array}{l}7.5 \\
4.0+1.6\end{array}$ & $\begin{array}{l}356 \\
32+-\end{array}$ & 24 & $\begin{array}{l}7.7 \\
4.7\end{array}$ & $\begin{array}{l}+3.8 \\
+2.3\end{array}$ & $\begin{array}{r}348 \\
11\end{array}$ & $\begin{array}{l}15 \\
37\end{array}$ \\
\hline $\begin{array}{c}\text { C.PORPOISE } \\
43^{\circ} 13^{\prime} \mathrm{N} . \\
70^{\circ} 17^{\prime} \mathrm{H} .\end{array}$ & $\begin{array}{l}74 \\
74\end{array}$ & $\begin{array}{l}33 \\
68\end{array}$ & $\begin{array}{l}65 \\
30\end{array}$ & $\begin{array}{l}2.7+0.5 \\
1.1+0.3\end{array}$ & $\begin{array}{l}228+ \\
240+-\end{array}$ & $\begin{array}{l}11 \\
18\end{array}$ & $\begin{array}{l}5.7+0.7 \\
3.0+-0.5\end{array}$ & $\begin{array}{l}334 t- \\
337 t\end{array}$ & $\begin{array}{l}7 \\
9\end{array}$ & $\begin{array}{l}5.8 \\
3.0\end{array}$ & $\begin{array}{l}+2.6 \\
+1.1\end{array}$ & $\begin{array}{l}338 \\
338\end{array}$ & $\begin{array}{l}351 \\
357\end{array}$ \\
\hline $\begin{array}{l}\text { DOSTON L.S. } \\
42^{\circ} 20^{\prime} \mathrm{N} . \\
70^{\circ} 45^{\prime} \mathrm{H} .\end{array}$ & 369 & 2 & 31 & 4.1 & 200 & & 0.6 & 163 & & 4.1 & -0.4 & 200 & 83 \\
\hline
\end{tabular}

Instrunent type, wethod of analyele and source of data are listed alphabetically by otatlons In Table 3. If UAINOR < 0, ellipse rotates clockwise

- Data 1o fron two separace nooriage at different tines.

$\begin{array}{rrrr}8.4 & -1.6 & 286 & 328 \\ 14.5 & +0.8 & 152 & 290 \\ 10.2 & +6.3 & 14 & 276 \\ 87.0 & -11.4 & 340 & 282 \\ 78.4 & -4.6 & 322 & 287 \\ 44.4 & +5.9 & 327 & 291 \\ 53.6 & -9.2 & 360 & 302 \\ 62.3 & -14.8 & 350 & 302 \\ 44.6 & -6.5 & 349 & 308\end{array}$

51.4t $3.0-16.1+-1.1 \quad 355 t 3307+4$ $57.5+3.2-21.4+2.7$ 355t $2312+2$ $47.8+0.8-11.4+1.8 \quad 341+3 \quad 327+4$

$48.6+0.1-11.6+1.4 \quad 357+1$ 309+ 2 $56.6+3.1-24.3+-3.5 \quad 354 t 2$ 312t 3 $41.4+-12.2-15.6+-2.7 \quad 347+4 \quad 314 t-1$

$57.9+2.8-15.5+3.9 \quad 2+3 \quad 308+0$ $61.1+2.7-26.9+-1.6 \quad 3+1 \quad 321+2$ $49.5+2.8-13.9+-3.3 \quad 348+2 \quad 330+5$ $42^{\circ} 20^{\prime} \mathrm{N}$ 
Table 5. $M_{2}$ tidal current parameters-Continued

Table 5. M2 TIDAL CURRENT PARAMETERS--Continued

\begin{tabular}{|c|c|c|c|c|c|c|}
\hline STATION & RECORD & INSTR & ABOVE & & URIER & COEFFICIENTS \\
\hline LAT. & LENGTH & DEPTH & BOTTOM & EAST & PHASE & NORTH \\
\hline
\end{tabular}

\begin{tabular}{cccc}
\multicolumn{2}{c}{ CURRENT ELLIPSE } & PARAMETERS \\
UMAJOR & UMINOR & PHASE & ORIEN \\
$(\mathrm{CM} / \mathrm{SEC})$ & $(\mathrm{CM} / \mathrm{SEC})$ & (DEG-G) & (DEG-TRUE)
\end{tabular}

BAY OF FUNDY

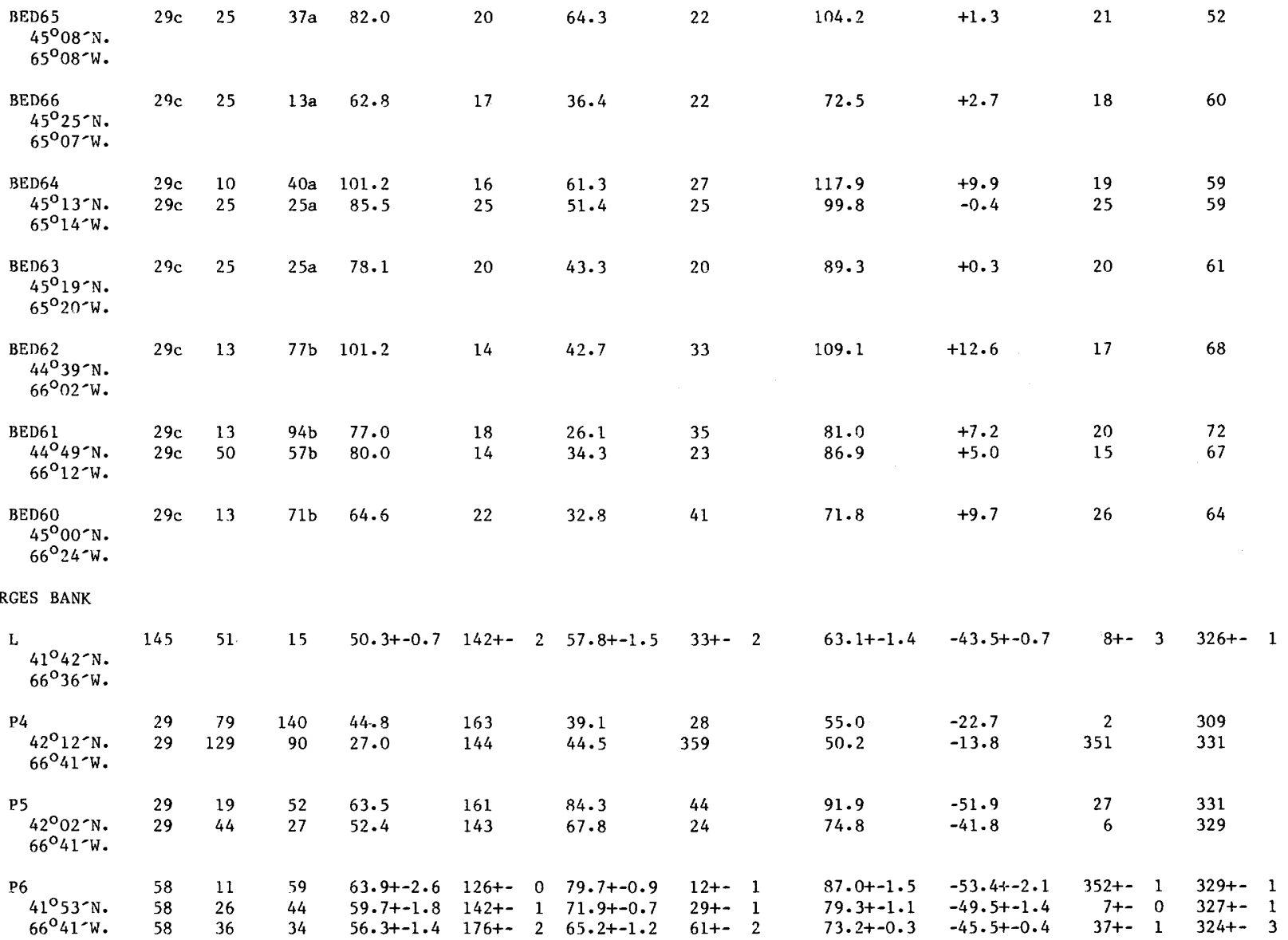

Instrument type, method of analysis and source of data are listed alphabetically by stations in Table 3 .

If UMINOR $<0$, ellipse rotates clockwise

a-water depth estimated from DriA chart 14040 (old 609)

b-water depth estimated from DMA chart 13102

c-estimated to be at least 29 -days 
Table 5. $\mathrm{M}_{2}$ tidal current parameters-Continued

Table 5. H2 TIDAL CURREMT PARARETERS-Continued

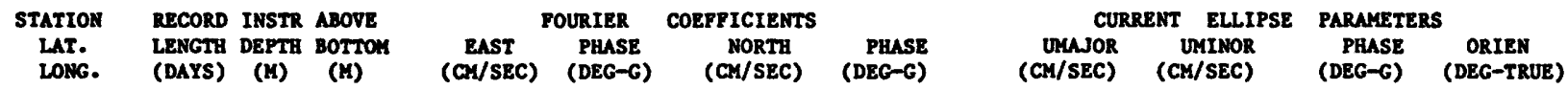

GEORGES BANK--CONT.

\begin{tabular}{|c|c|c|c|c|c|c|c|c|c|c|c|c|c|c|}
\hline $\begin{array}{l}M 1^{\star *} \\
42^{\circ} 04 ' \mathrm{~N} . \\
67^{\circ} 52^{\prime} \mathrm{H} .\end{array}$ & $\underset{\mathbf{M A}}{\mathbf{M}}$ & $\begin{array}{r}77 \\
192\end{array}$ & $\begin{array}{r}123 \\
8\end{array}$ & $\begin{array}{r}10.9 \\
9.4\end{array}$ & $\begin{array}{r}164 \\
89\end{array}$ & & $\begin{array}{r}7.1 \\
21.1\end{array}$ & $\begin{array}{r}20 \\
311\end{array}$ & & $\begin{array}{l}12.5 \\
22.3\end{array}$ & $\begin{array}{l}-3.6 \\
-5.9\end{array}$ & $\begin{array}{l}354 \\
305\end{array}$ & & $\begin{array}{l}301 \\
340\end{array}$ \\
\hline $\begin{array}{l}84^{*} \\
40^{\circ} 56 \text { ' N. } \\
66^{\circ} 58^{\prime} \mathrm{W} .\end{array}$ & $\begin{array}{l}58 \\
58 \\
58\end{array}$ & $\begin{array}{l}10 \\
36 \\
59\end{array}$ & $\begin{array}{r}67 \\
41 \\
8\end{array}$ & $\begin{array}{l}30.6 \\
28.8 \\
20.8\end{array}$ & $\begin{array}{l}158 \\
151 \\
123\end{array}$ & & $\begin{array}{l}36.0 \\
32.7 \\
26.9\end{array}$ & $\begin{array}{l}46 \\
41 \\
11\end{array}$ & & $\begin{array}{l}39.5+-2.2 \\
36.0+-1.5 \\
28.8+-2.2\end{array}$ & $\begin{array}{l}-25.9+-2.0 \\
-24.5+1.7 \\
-18.1+1.6\end{array}$ & $\begin{array}{c}23+ \\
15+ \\
353+\end{array}$ & $\begin{array}{l}3 \\
2 \\
4\end{array}$ & $\begin{array}{l}327 \\
325 \\
333\end{array}$ \\
\hline $\begin{array}{l}\mathrm{P1} \\
\quad 42^{\circ} 12^{\prime} \mathrm{N} . \\
67^{\circ} 15^{\prime} \mathrm{W} .\end{array}$ & $\begin{array}{l}15 \\
15 \\
15\end{array}$ & $\begin{array}{l}30 \\
40 \\
75\end{array}$ & $\begin{array}{l}173 \\
163 \\
128\end{array}$ & $\begin{array}{l}47.7 \\
46.4 \\
35.7\end{array}$ & $\begin{array}{l}195 \\
197 \\
154\end{array}$ & & $\begin{array}{l}24.6 \\
22.1 \\
31.7\end{array}$ & $\begin{array}{l}95 \\
98 \\
18\end{array}$ & & $\begin{array}{l}47.9 \\
46.6 \\
44.3\end{array}$ & $\begin{array}{l}-24.1 \\
-21.7 \\
-17.7\end{array}$ & $\begin{array}{r}18 \\
19 \\
353\end{array}$ & & $\begin{array}{l}277 \\
276 \\
310\end{array}$ \\
\hline $\begin{array}{l}P 2 \\
42^{\circ} 03 \text { 'N. } \\
67^{\circ} 15^{\prime} \mathrm{W} .\end{array}$ & $\begin{array}{l}30 \\
15\end{array}$ & $\begin{array}{l}14 \\
30\end{array}$ & $\begin{array}{l}36 \\
20\end{array}$ & $\begin{array}{l}55.6+6.8 \\
46.7\end{array}$ & $\begin{array}{l}151+ \\
145\end{array}$ & 6 & $\begin{array}{l}89.8+1.7 \\
77.0\end{array}$ & $\begin{array}{l}13+ \\
10\end{array}$ & 3 & $\begin{array}{l}100.4+-2.1 \\
84.8\end{array}$ & $\begin{array}{l}-33.0+0.7 \\
-30.0\end{array}$ & $360^{3+}$ & 1 & $\begin{array}{l}332+ \\
333\end{array}$ \\
\hline $\begin{array}{l}\text { P3 } \\
\begin{array}{l}1^{\circ} 553^{\prime} \mathrm{N} . \\
67^{\circ} 15^{\prime} \mathrm{W} .\end{array}\end{array}$ & $\begin{array}{l}15 \\
15 \\
15\end{array}$ & $\begin{array}{l}15 \\
30 \\
40\end{array}$ & $\begin{array}{r}30 \\
15 \\
5\end{array}$ & $\begin{array}{l}54.0 \\
51.8 \\
30.1\end{array}$ & $\begin{array}{l}116 \\
132 \\
136\end{array}$ & & $\begin{array}{l}91.7 \\
83.2 \\
56.9\end{array}$ & $\begin{array}{l}14 \\
17 \\
44\end{array}$ & & $\begin{array}{l}94.4 \\
87.1 \\
56.9\end{array}$ & $\begin{array}{l}-49.5 \\
-44.9 \\
-30.1\end{array}$ & $\begin{array}{r}8 \\
7 \\
43\end{array}$ & & $\begin{array}{l}349 \\
340 \\
359\end{array}$ \\
\hline $\begin{array}{l}M 3^{*} \\
41^{\circ} 0_{20} \mathrm{~N} . \\
67^{\circ} 16^{\prime} \mathrm{W} .\end{array}$ & 58 & 36 & 8 & 46.7 & 129 & & 55.1 & 22 & & $58.8+-1.0$ & $-41.9+-0.9$ & $2+$ & 1 & 330 \\
\hline $\begin{array}{l}49^{*} \\
40^{\circ} 54 \cdot \mathrm{N} . \\
67^{\circ} 24 \mathrm{~W} .\end{array}$ & 58 & 71 & 8 & 19.7 & 115 & & 32.1 & 6 & & $33.0+-1.4$ & $-18.1+1.1$ & $357+$ & 2 & 344 \\
\hline $\begin{array}{l}40^{\circ} 51^{\prime} \mathrm{N} . \\
67^{\circ} 24^{\prime} \mathrm{W} \text {. }\end{array}$ & $\begin{array}{l}261 \\
957 \\
957 \\
290\end{array}$ & $\begin{array}{l}15 \\
45 \\
75 \\
84\end{array}$ & $\begin{array}{r}70 \\
40 \\
10 \\
1\end{array}$ & $\begin{array}{l}27.5+-1.7 \\
29.4+1.8 \\
22.9+-1.5 \\
14.1+1.4\end{array}$ & $\begin{array}{l}137+ \\
134 t \\
119+ \\
108+\end{array}$ & $\begin{array}{r}4 \\
4 \\
4 \\
10\end{array}$ & $\begin{array}{l}34.2+-2.6 \\
35.8+1.4 \\
30.6+1.9 \\
21.0+1.2\end{array}$ & $\begin{array}{c}27+ \\
26+ \\
8+ \\
360+\end{array}$ & $\begin{array}{l}2 \\
4 \\
5 \\
6\end{array}$ & $\begin{array}{l}36.6+-2.4 \\
38.3+-1.6 \\
32.5+-2.0 \\
21.8+-1.5\end{array}$ & $\begin{array}{l}-24.2+-1.9 \\
-26.0+1.6 \\
-20.1+1.3 \\
-12.9+0.9\end{array}$ & $\begin{array}{c}8+ \\
5+ \\
352+ \\
349+\end{array}$ & $\begin{array}{l}2 \\
2 \\
3 \\
3\end{array}$ & $\begin{array}{l}332+ \\
331+ \\
335+ \\
341+\end{array}$ \\
\hline $\begin{array}{l}{ }^{4} 1^{\circ} 24 \text { 'N. } \\
67^{\circ} 34 \text { 'W. }\end{array}$ & 116 & 25 & 23 & $59.0+-0.7$ & $122+$ & 1 & $71.6+-0.2$ & $20+$ & 1 & $74.3+-0.4$ & $-55.6+-0.5$ & $2+-$ & 1 & $336+$ \\
\hline $\begin{array}{l}\mathrm{x}^{* * *} \\
41^{\circ} 04 \text { ' } \mathrm{k} . \\
67^{\circ} 34^{\prime} \mathrm{W} .\end{array}$ & $\begin{array}{r}58 \\
174 \\
58 \\
174 \\
87 \\
232\end{array}$ & $\begin{array}{l}10 \\
15 \\
34 \\
54 \\
58 \\
60\end{array}$ & $\begin{array}{r}54 \\
45 \\
30 \\
10 \\
4 \\
1\end{array}$ & $\begin{array}{l}43.8+-0.8 \\
42.2+-0.5 \\
43.8+-0.8 \\
31.8+-0.8 \\
30.3+-0.7 \\
22.1+-1.3\end{array}$ & $\begin{array}{l}142+ \\
139+ \\
142+ \\
125+ \\
129+ \\
116+\end{array}$ & $\begin{array}{l}0 \\
4 \\
0 \\
4 \\
1 \\
6\end{array}$ & $\begin{array}{l}51.1+-0.3 \\
49.6+-1.9 \\
51.1+-0.3 \\
41.9+-0.8 \\
38.7+-1.1 \\
30.4+-0.9\end{array}$ & $\begin{array}{l}34+ \\
30+ \\
34+ \\
15+ \\
19+ \\
8+\end{array}$ & $\begin{array}{l}1 \\
3 \\
1 \\
5 \\
1 \\
3\end{array}$ & $\begin{array}{l}55.1+-0.0 \\
53.6+-1.3 \\
55.1+-0.0 \\
44.4+-0.9 \\
41.2+1.1 \\
31.8+-1.0\end{array}$ & $\begin{array}{l}-38.7+-1.2 \\
-37.0+-1.1 \\
-38.7+-1.2 \\
-28.2+-1.2 \\
-26.8+-0.6 \\
-20.2+-1.1\end{array}$ & $\begin{array}{c}11+ \\
7+ \\
11+ \\
359+ \\
1+ \\
354+-\end{array}$ & $\begin{array}{l}1 \\
1 \\
1 \\
5 \\
1 \\
3\end{array}$ & $\begin{array}{l}328+ \\
328+ \\
328+ \\
335+ \\
333+ \\
339+\end{array}$ \\
\hline
\end{tabular}

Instrument type, wethod of analysis and source of data are liated alphabetically by stations in Table 3.

If UMINOR < 0, ellipse rotates clockwse

* - depthe are besed on wean pressure and error estinates are taken from acrose-1sobath and along-1sobsth directions ince these are neariy the sane as the ellipse axis. These records span identical time periods.

** -data taken from figure 8 in Magnel1,1980.

th*-tripod and subsurface moorings were at different depths 
Table 5. $M_{2}$ tidal current parameters-Continued

Table 5. M2 TIDAL CURRENT PARAMETERS--Continued

\begin{tabular}{|c|c|c|c|c|c|c|c|}
\hline ТА' & ECORD & INSTR & ABOVE & & URIER & COEFFICLENTS & \\
\hline $\begin{array}{l}\text { LAT. } \\
\text { LONG. }\end{array}$ & $\begin{array}{l}\text { LENGTH } \\
\text { (DAYS) }\end{array}$ & $\begin{array}{l}\text { DEPTH } \\
\text { (M) }\end{array}$ & $\begin{array}{l}\text { BOTTOM } \\
\text { (M) }\end{array}$ & $\begin{array}{c}\text { EAST } \\
(\mathrm{CM} / \mathrm{SEC})\end{array}$ & $\begin{array}{c}\text { PHASE } \\
(\text { DEG }-G)\end{array}$ & $\begin{array}{c}\text { NORTH } \\
(\mathrm{CM} / \mathrm{SEC})\end{array}$ & $\begin{array}{c}\text { PHASE } \\
\text { (DEG-G) }\end{array}$ \\
\hline
\end{tabular}

\begin{tabular}{lccc}
\multicolumn{2}{c}{ CURRENT } & ELLIPSE & PARAMETERS \\
UMAJOR & UMINOR & PHASE & ORIEN \\
$($ CM/SEC $)$ & $($ CM/SEC $)$ & (DEG-G) & (DEG-TRUE)
\end{tabular}

GEORGES BANK--CONT.

D $41^{\mathrm{O}} 59-\mathrm{N}$
$67^{\circ} 47^{-\mathrm{W}}$

M2 $42^{\circ} 00^{-}$

NA $\quad 77$

$8 \quad 22.2$

113
61

$$
\begin{aligned}
& 27.7 \\
& 29.0
\end{aligned}
$$

29.0

2
308

GREAT SOUTH CHANNEL

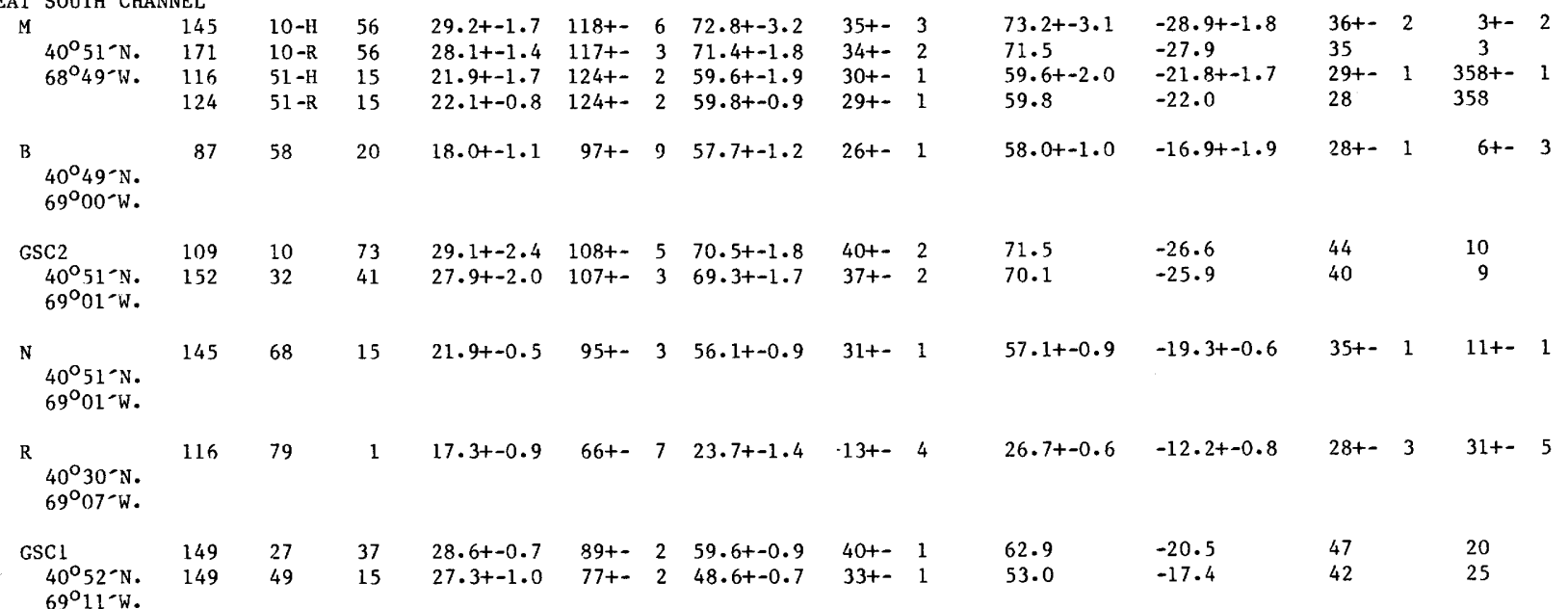

LYDONIA CANYON ${ }^{* *}$

\begin{tabular}{|c|c|c|}
\hline LCA & 116 & 80 \\
\hline $\begin{array}{l}40^{\circ} 34^{-} \mathrm{N} \\
67^{\circ} 45^{-} \mathrm{W}\end{array}$ & 58 & 99 \\
\hline LCB & 145 & 92 \\
\hline $40^{\circ} 32^{-} \mathrm{N}$ & 145 & 227 \\
\hline $67^{\circ} 43^{-W}$. & 116 & 277 \\
\hline
\end{tabular}

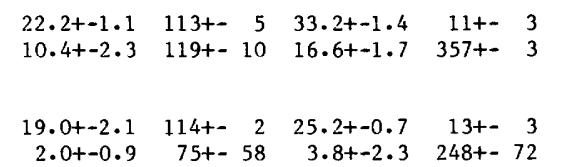

Instrument type, method of analysis and source of data are listed alphabetically by stations in Table 3 .

If UMINOR $<0$, ellipse rotates clockwise

$\mathrm{H}=$ harmonic analysis

$\mathrm{R}=$ response analysis

* - Data taken from Figure 8 in Magne11,1980.

** - Observations from stations LCF, except $277 \mathrm{~m}$ ), LCE, LCH(except 1375 and $1454 \mathrm{~m}$ ), LCI, LCJ(except 454m), and LCN al1 begin at 0100 on Dec.2,1980 and end at 0100 on April 26,1981. Observations at LCA(80m),LCB(277m),LCJ(83m), LCK( $454 \mathrm{~m})$ and LCL begin at 0100 on Dec.2,1980 but end 29 days earlier on March 28, 1981 . 
Table 5. $M_{2}$ tidal current parameters-Continued

Table 5. M2 TIDAL CURRENT PARAMETERS--Continued

\begin{tabular}{cllcccrr} 
STATION & RECORD INSTR ABOVE & \multicolumn{3}{c}{ FOURIER } & COEFFICIENTS \\
LAT. & LENGTH & DEPTH & BOTTOM & EAST & PHASE & NORTH & PHASE \\
LONG. & (DAYS) & (M) & (M) & (CM/SEC) & (DEG-G) & $($ CM/SEC) & (DEG-G)
\end{tabular}

\begin{tabular}{cccc}
\multicolumn{2}{c}{ CURRENT } & ELLIPSE & PARAMETERS \\
UMAJOR & UMINOR & PHASE & ORIEN \\
$(\mathrm{CM} / \mathrm{SEC})$ & $(\mathrm{CM} / \mathrm{SEC})$ & (DEG-G) & (DEG-TRUE)
\end{tabular}

LYDONIA CANYON--CONT.

\begin{tabular}{|c|c|c|c|c|c|c|c|c|c|c|c|c|c|c|}
\hline $\begin{array}{l}\mathrm{LCC} \\
\quad 40^{\circ} 29^{\circ} \mathrm{N} . \\
67^{\circ} 44^{-} \mathrm{W} .\end{array}$ & 116 & 134 & 50 & $9.1+-2.4$ & $113+-$ & 4 & $15 \cdot 6+-3.5$ & $2+-$ & 2 & $16.2+-3.6$ & $-8 \cdot 1+-2.1$ & $353+-$ & 3 & $343+-$ \\
\hline $\begin{array}{l}\text { LCD } \\
\qquad 0^{\circ} 29^{-} \mathrm{N} . \\
67^{\circ} 41^{-W} .\end{array}$ & 116 & 143 & 50 & $9.9+-1.8$ & $96+-$ & 13 & $22.5+-2 \cdot 1$ & $358+-$ & 8 & $22 \cdot 6+-2 \cdot 2$ & $-9.7+-1.7$ & $356+-$ & 7 & $356+-$ \\
\hline $\begin{array}{l}\text { LCE } \\
\qquad \begin{array}{l}40^{\circ} 25^{-} \mathrm{N} \\
67^{\circ} 40^{-} \mathrm{W} .\end{array}\end{array}$ & $\begin{array}{l}145 \\
145 \\
145\end{array}$ & $\begin{array}{l}116 \\
216 \\
595\end{array}$ & $\begin{array}{r}484 \\
384 \\
5\end{array}$ & $\begin{array}{r}11.6+-2.7 \\
5.3+-0.8 \\
6.0+-0.6\end{array}$ & $\begin{array}{r}125+- \\
10+- \\
21+-\end{array}$ & $\begin{array}{r}6 \\
22 \\
12\end{array}$ & $\begin{array}{l}18 \cdot 9+-1 \cdot 9 \\
11 \cdot 8+-0.8 \\
14 \cdot 7+-1 \cdot 2\end{array}$ & $\begin{array}{r}23+- \\
7+- \\
24+-\end{array}$ & $\begin{array}{r}4 \\
5 \\
13\end{array}$ & $\begin{array}{l}19.1+-2.0 \\
12.9+-0.7 \\
15.8+-1.3\end{array}$ & $\begin{array}{l}-11.2+-2.7 \\
-0.3+-1.7 \\
+0.3+-0.6\end{array}$ & $\begin{array}{r}17+- \\
8+- \\
23+-\end{array}$ & $\begin{array}{r}4 \\
7 \\
13\end{array}$ & $\begin{array}{r}350+- \\
24+- \\
22+-\end{array}$ \\
\hline $\begin{array}{l}\text { LCF } \\
\qquad \begin{array}{l}40^{\circ} 21-\mathrm{N} \\
67^{\circ} 39^{-} \mathrm{W}\end{array}\end{array}$ & $\begin{array}{l}145 \\
174\end{array}$ & $\begin{array}{l}205 \\
405\end{array}$ & $\begin{array}{l}300 \\
100\end{array}$ & $\begin{array}{l}6.4+-1.6 \\
5.4+-0.8\end{array}$ & $\begin{array}{l}104+- \\
355+-\end{array}$ & $\begin{array}{r}25 \\
7\end{array}$ & $\begin{array}{l}8.8+-1.4 \\
4.4+-0.4\end{array}$ & $\begin{array}{r}12+- \\
358+-\end{array}$ & $\begin{array}{r}15 \\
9\end{array}$ & $\begin{array}{l}8.9+-1.5 \\
7.0+-0.8\end{array}$ & $\begin{array}{l}-6.2+-1.5 \\
+0.2+-0.4\end{array}$ & $\begin{array}{r}12+- \\
356+-\end{array}$ & $\begin{array}{r}17 \\
7\end{array}$ & $\begin{array}{r}360+- \\
51+-\end{array}$ \\
\hline $\begin{array}{l}\text { LCG } \\
\quad 40^{\circ} 21^{-} \mathrm{N} . \\
67^{\circ} 42^{-} \mathrm{W} .\end{array}$ & $\begin{array}{l}174 \\
174\end{array}$ & $\begin{array}{l}195 \\
395\end{array}$ & $\begin{array}{l}300 \\
100\end{array}$ & $\begin{array}{l}6.7+-1.0 \\
3.1+-0.5\end{array}$ & $\begin{array}{l}85+- \\
21+-\end{array}$ & $\begin{array}{r}23 \\
9\end{array}$ & $\begin{array}{l}6.9+-2.2 \\
4.2+-0.6\end{array}$ & $\begin{array}{r}14+- \\
357+-\end{array}$ & $\begin{array}{r}26 \\
6\end{array}$ & $\begin{array}{l}8.6+-0.8 \\
5.1+-0.7\end{array}$ & $\begin{array}{l}-4.6+-1.7 \\
-1.0+-0.2\end{array}$ & $\begin{array}{r}24+- \\
5+-\end{array}$ & $\begin{array}{r}45 \\
8\end{array}$ & $\begin{array}{l}24+- \\
36+-\end{array}$ \\
\hline $\begin{array}{l}\mathrm{LCH}^{*} \\
\quad 40^{\circ} 18^{-} \mathrm{N} . \\
67^{\circ} 40^{-} \mathrm{W} .\end{array}$ & $\begin{array}{r}145 \\
145 \\
145 \\
29 \\
87\end{array}$ & $\begin{array}{r}290 \\
540 \\
890 \\
1454 \\
1375\end{array}$ & $\begin{array}{r}1264 \\
1014 \\
664 \\
100 \\
5\end{array}$ & $\begin{array}{l}4.3+-1.5 \\
1.6+-0.4 \\
1.4+-0.3 \\
1.2 \\
1.1+-0.1\end{array}$ & $\begin{array}{r}131+- \\
31+- \\
188+- \\
25 \\
101+-\end{array}$ & $\begin{array}{r}9 \\
23 \\
7 \\
28\end{array}$ & $\begin{array}{l}6.9+-2.2 \\
3.3+-1.0 \\
3.5+-0.5 \\
5.5 \\
5.8+-0.9\end{array}$ & $\begin{array}{c}21+- \\
312+- \\
2+- \\
342 \\
332+-\end{array}$ & $\begin{array}{l}11 \\
20 \\
17 \\
19\end{array}$ & $\begin{array}{l}7.1+-2.1 \\
3.4+-0.9 \\
3.8+-0.5 \\
5.5 \\
5.8+-0.9\end{array}$ & $\begin{array}{l}-4.0+-1.6 \\
-1.5+-0.4 \\
+0.1+-0.3 \\
-0.8 \\
-0.8+-0.3\end{array}$ & $\begin{array}{r}13+-1 \\
307+-4 \\
3+-1 \\
343 \\
331+-1\end{array}$ & $\begin{array}{l}13 \\
43 \\
16 \\
19\end{array}$ & $\begin{array}{c}344+- \\
355+- \\
339+- \\
10 \\
354+-\end{array}$ \\
\hline $\begin{array}{l}\text { LCI } \\
\quad 40^{\circ} 23^{-} \mathrm{N} . \\
67^{\circ} 33^{-1} .\end{array}$ & $\begin{array}{l}145 \\
145 \\
145 \\
145\end{array}$ & $\begin{array}{r}10 \\
55 \\
195 \\
245\end{array}$ & $\begin{array}{r}240 \\
195 \\
55 \\
5\end{array}$ & $\begin{array}{r}10.0+-3.9 \\
9.1+-2.7 \\
9.9+-3.6 \\
3 . .8+-0.7\end{array}$ & $\begin{array}{r}141+- \\
125+- \\
106+- \\
57+-\end{array}$ & $\begin{array}{l}19 \\
16 \\
12 \\
23\end{array}$ & $\begin{array}{r}11.6+-2.5 \\
10.8+-1.8 \\
9.3+-1.9 \\
6.0+-1.6\end{array}$ & $\begin{array}{r}45+- \\
35+- \\
18+- \\
316+-\end{array}$ & $\begin{array}{r}20 \\
17 \\
6 \\
19\end{array}$ & $\begin{array}{r}11.8+-2.6 \\
11.2+-1.9 \\
11.0+-3.8 \\
6.3+-1.1\end{array}$ & $\begin{array}{l}-9.6+-3.8 \\
-8.7+-2.3 \\
-8.0+-1.3 \\
-3.3+-0.5\end{array}$ & $\begin{array}{r}34+-3 \\
46+-4 \\
358+-4 \\
302+-3\end{array}$ & $\begin{array}{l}30 \\
44 \\
49 \\
33\end{array}$ & $\begin{array}{r}349+- \\
12+- \\
342+- \\
340+-\end{array}$ \\
\hline $\begin{array}{l}\text { LCJ } \\
\qquad \begin{array}{l}40^{\circ} 21-\mathrm{N} . \\
67^{\circ} 32-\mathrm{W} .\end{array}\end{array}$ & $\begin{array}{l}116 \\
145 \\
145\end{array}$ & $\begin{array}{r}83 \\
223 \\
471\end{array}$ & $\begin{array}{l}488 \\
348 \\
100\end{array}$ & $\begin{array}{l}6.4+-2.0 \\
4.9+-2.6 \\
1.2+-0.4\end{array}$ & $\begin{array}{r}129+- \\
127+- \\
5+-\end{array}$ & $\begin{array}{l}20 \\
11 \\
92\end{array}$ & $\begin{array}{l}9.1+-2.7 \\
7.2+-2.6 \\
1.6+-0.6\end{array}$ & $\begin{array}{r}45+- \\
30+- \\
227+-\end{array}$ & $\begin{array}{l}13 \\
11 \\
18\end{array}$ & $\begin{array}{l}9.1+-2.7 \\
7.3+-2.6 \\
1.8+-0.6\end{array}$ & $\begin{array}{l}-6.2+-2.1 \\
-4.8+-2.6 \\
=0.5+-0.7\end{array}$ & $\begin{array}{c}50+- \\
24+-1 \\
212+-2\end{array}$ & $\begin{array}{r}8 \\
11 \\
21\end{array}$ & $\begin{array}{r}8+- \\
351+- \\
340+-\end{array}$ \\
\hline $\begin{array}{l}\text { LCK } \\
\quad 40^{\circ} 16^{-} \mathrm{N} . \\
67^{\circ} 47^{-} \mathrm{W} .\end{array}$ & $\begin{array}{l}145 \\
145\end{array}$ & $\begin{array}{l}204 \\
454\end{array}$ & $\begin{array}{l}350 \\
100\end{array}$ & $\begin{array}{l}4.0+-1.7 \\
4.3+-1.3\end{array}$ & $\begin{array}{r}146+- \\
61+-\end{array}$ & $\begin{array}{l}10 \\
22\end{array}$ & $\begin{array}{l}5.5+-1.6 \\
4.3+-1.6\end{array}$ & $\begin{array}{r}47+- \\
296+-\end{array}$ & $\begin{array}{r}6 \\
18\end{array}$ & $\begin{array}{l}6.6+-1.6 \\
5.5+-1.7\end{array}$ & $\begin{array}{l}-3.9+-1.7 \\
-2.6+-1.0\end{array}$ & $\begin{array}{c}42+- \\
263+-2\end{array}$ & $\begin{array}{r}6 \\
21\end{array}$ & $\begin{array}{l}352+- \\
311+-\end{array}$ \\
\hline $\begin{array}{l}\text { LCI, } \\
\qquad 40^{\circ} 32^{-} \mathrm{N} . \\
67^{\circ} 36^{-} \mathrm{W}\end{array}$ & $\begin{array}{l}116 \\
116\end{array}$ & $\begin{array}{r}65 \\
105\end{array}$ & $\begin{array}{l}60 \\
20\end{array}$ & $\begin{array}{l}18.7+-1.3 \\
19.4+-3.7\end{array}$ & $\begin{array}{l}121+- \\
106+-\end{array}$ & $\begin{array}{l}2 \\
4\end{array}$ & $\begin{array}{l}22.9+-0.7 \\
22.6+-1.2\end{array}$ & $\begin{array}{r}21+- \\
4+-\end{array}$ & $\begin{array}{l}2 \\
2\end{array}$ & $\begin{array}{l}23.5+-0.7 \\
23.9+-1.9\end{array}$ & $\begin{array}{l}-18 \cdot 0+-1 \cdot 3 \\
-17 \cdot 9+-2.9\end{array}$ & $\begin{array}{c}6+- \\
341+-1\end{array}$ & $\begin{array}{l}2 \\
17\end{array}$ & $\begin{array}{l}340+- \\
332+-\end{array}$ \\
\hline $\begin{array}{l}\mathrm{LCM}^{*} \\
\quad 40^{\circ} 30^{-\mathrm{N}} . \\
67^{\circ} 49^{-\mathrm{W}} .\end{array}$ & $\begin{array}{l}145 \\
174\end{array}$ & $\begin{array}{l}103 \\
119\end{array}$ & $\begin{array}{r}20 \\
1\end{array}$ & $\begin{array}{l}18.8+-1.8 \\
10.4+-2.5\end{array}$ & $\begin{array}{r}100+- \\
85+-\end{array}$ & $\begin{array}{r}6 \\
11\end{array}$ & $\begin{array}{l}25.5+-2.5 \\
15.1+-3.7\end{array}$ & $\begin{array}{r}6+- \\
344+-\end{array}$ & $\begin{array}{l}5 \\
8\end{array}$ & $\begin{array}{l}25.6+-2.5 \\
15.5+-3.6\end{array}$ & $\begin{array}{r}-18.6+-1.9 \\
-9.9+-2.4\end{array}$ & $\begin{array}{r}2+- \\
333+-\end{array}$ & $\begin{array}{r}7 \\
11\end{array}$ & $\begin{array}{l}354+- \\
344+-\end{array}$ \\
\hline $\begin{array}{l}\mathrm{LCN} \\
\quad 40^{\circ} 21-\mathrm{N} .\end{array}$ & $\begin{array}{l}145 \\
145\end{array}$ & $\begin{array}{l}243 \\
841\end{array}$ & $\begin{array}{l}798 \\
200\end{array}$ & $\begin{array}{l}6.2+-0.6 \\
4.9+-1.1\end{array}$ & $\begin{array}{r}105+- \\
5+-\end{array}$ & $\begin{array}{r}10 \\
4\end{array}$ & $\begin{array}{l}7.4+-2.9 \\
7.0+-1.4\end{array}$ & $\begin{array}{r}17+- \\
347+-\end{array}$ & $\begin{array}{r}31 \\
7\end{array}$ & $\begin{array}{l}8.6+-1.3 \\
8.4+-1.7\end{array}$ & $\begin{array}{l}-4.8+-1.8 \\
-1.2+-0.3\end{array}$ & $\begin{array}{r}15+- \\
353+-\end{array}$ & $\begin{array}{r}55 \\
6\end{array}$ & $\begin{array}{r}4+- \\
34+-\end{array}$ \\
\hline
\end{tabular}
$67^{\circ} 40 \%$ W.

Instrument type, method of analysis and source of data are listed alphabetically by stations in Table 3 .

If UMINOR < 0 , ellipse rotates clockwise

* - Two separate moorings at different depths 
Table 5. $M_{2}$ tidal current parameters-Continued

Table 5. M2 TIDAL CURRENT PARAMETERS--Continued

STATION RECORD INSTR ABOVE

LAT. LENGTH DEPTH BOTTOM

LONG. (DAYS) (M) (M)

NANTUCKET SHOALS

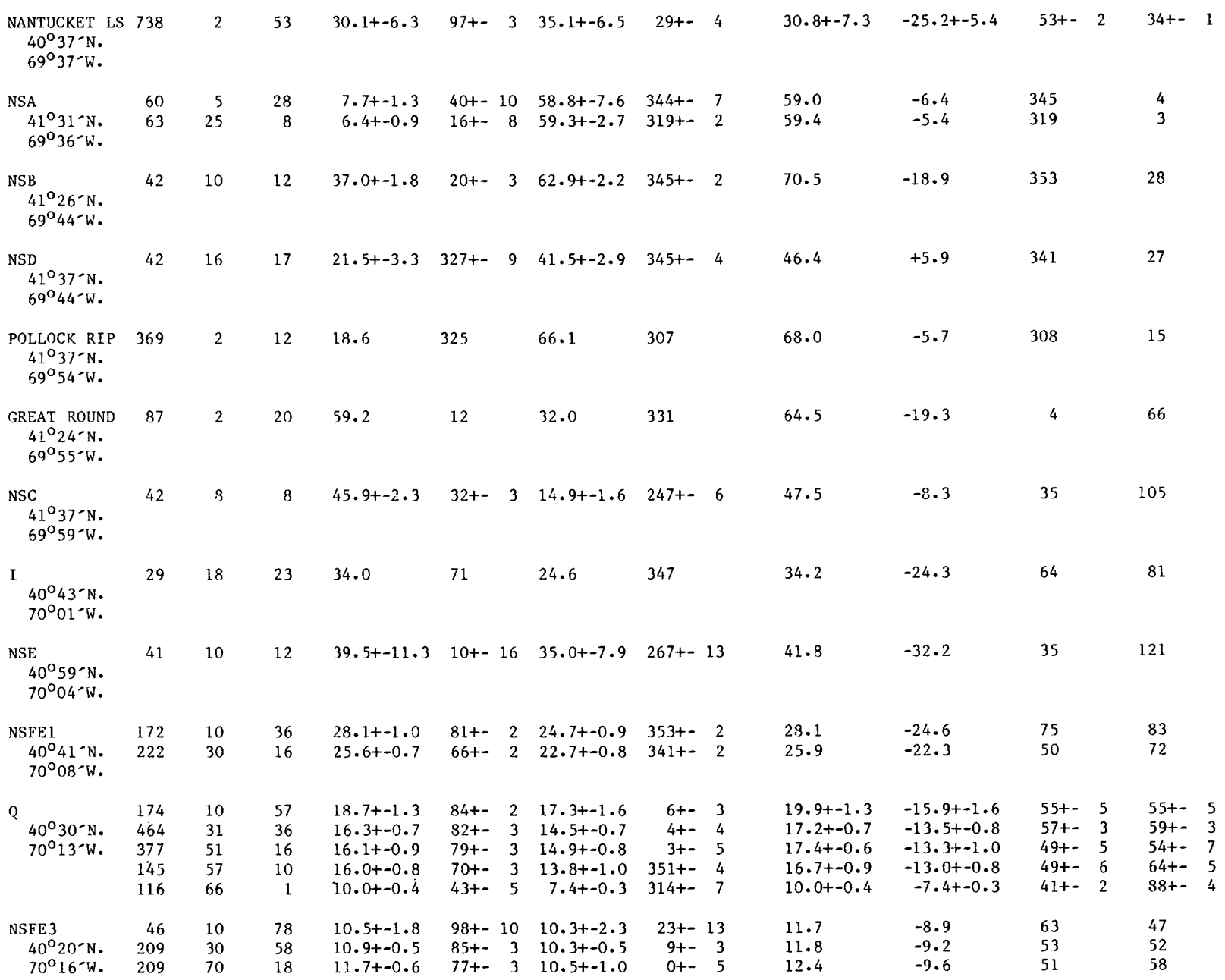

Instrument type, method of analysis and source of data are listed alphabetically by stations in Table 3 .

[f UMINOR $<0$, ellipse rotates clockwise 
Table 5. $\mathrm{M}_{2}$ tidal current parameters-Continued

Table 5. M2 TIDAL CURRENT PARAMETERS--Continued

\begin{tabular}{cllccccr} 
STATION & RECORD & INSTR ABOVE & \multicolumn{3}{c}{ FOURIER } & COEFFICIENTS \\
LAT. & LENGTH & DEPTH & BOTTOM & EAST & PHASE & NORTH & PHASE \\
LONG. & (DAYS $)$ & (M) & (M) & (CM/SEC) & $($ DEG-G) & (CM/SEC) & (DEG-G)
\end{tabular}

CURRENT ELLIPSE
UMAJOR
$\begin{array}{cc}\text { UMINOR } \\ (\mathrm{CM} / \mathrm{SEC}) & (\mathrm{CM} / \mathrm{SEC})\end{array}$

PARAMETERS
PHASE
$($ ORG $-G) \quad$ (DEG-TRUE

NANTUCKET SHOALS--CONT.

\begin{tabular}{|c|c|c|c|c|c|c|c|c|}
\hline $\begin{array}{l}\text { NSFE4 } \\
\quad 40^{\circ} 13^{-} \mathrm{N} . \\
70^{\circ} 18^{-} \mathrm{W} .\end{array}$ & $\begin{array}{l}170 \\
224 \\
170 \\
224\end{array}$ & $\begin{array}{l}10 \\
30 \\
60 \\
90\end{array}$ & $\begin{array}{l}95 \\
75 \\
45 \\
15\end{array}$ & $\begin{array}{r}10.0+-1.6 \\
8.1+-0.8 \\
7.7+-0.7 \\
8.0+-0.9\end{array}$ & $\begin{array}{l}96+- \\
94+- \\
87+- \\
78+-\end{array}$ & $\begin{array}{l}9 \\
5 \\
5 \\
6\end{array}$ & $\begin{array}{r}10.9+-1.7 \\
8.1+-0.8 \\
7.1+-0.6 \\
8.0+-1.0\end{array}$ & $\begin{array}{r}15+- \\
13+- \\
7+- \\
3+-\end{array}$ \\
\hline $\begin{array}{l}\text { NSFES } \\
\quad 40^{\circ} \mathrm{O} 2^{-N} \text {. } \\
70^{\circ} 22^{-} \mathrm{W} .\end{array}$ & $\begin{array}{l}171 \\
171 \\
171 \\
171 \\
171\end{array}$ & $\begin{array}{r}10 \\
30 \\
90 \\
120 \\
185\end{array}$ & $\begin{array}{r}188 \\
168 \\
108 \\
78 \\
13\end{array}$ & $\begin{array}{l}3.2+-1.4 \\
2.7 \\
3.4+-1.1 \\
4.4+-0.9 \\
4.1+-1.2\end{array}$ & $\begin{array}{c}129+- \\
109 \\
75+- \\
71+- \\
102+-\end{array}$ & $\begin{array}{l}19 \\
12 \\
17\end{array}$ & $\begin{array}{l}2.8+-1.3 \\
2.3 \\
2.6+-1.1 \\
3.9+-1.0 \\
4.1+-1.2\end{array}$ & $\begin{array}{c}50+- \\
28 \\
355+- \\
347+- \\
24+-\end{array}$ \\
\hline $\begin{array}{l}\text { NSFE6 } \\
\quad 39^{\circ} 51^{-} \mathrm{N} . \\
70^{\circ} 25^{-} \mathrm{W} .\end{array}$ & 225 & 10 & 800 & $1.6+-0.9$ & $236+-$ & 33 & $2.4 t-1.1$ & $148+-$ \\
\hline
\end{tabular}

11.3

8.7

8.1

9.0

3.4

2.8

3.4

4.5
4.5

2.4

NEW ENGLAND SHELF

P $\begin{array}{llrr}40^{\circ} 29^{-} \mathrm{N} . & 87 & 61 & 10 \\ 70^{\circ} 30^{-} \mathrm{W} . & 58 & 70 & 1\end{array}$

NES743 $40^{\circ} 18^{-\mathrm{N}}$ $70^{\circ} 52-\mathrm{W}$.

NES764 390051995 $70^{\circ} 56^{-} \mathrm{W}$

NES742 $40^{\circ} 35^{-} \mathrm{N}$ $79^{\circ} 59^{-} \mathrm{W}$.

VINEYARD $41^{\circ} 23^{-} \mathrm{N}$. $71^{\circ} 00^{\circ} \mathrm{W}$.

HENS\&CHICK 369 $41^{\circ} 27^{-} \mathrm{N}$. $71^{\circ} 01^{-W}$.

NES763 $39^{\circ} 56^{-} \mathrm{N}$. $71^{\circ} 03^{-} \mathrm{W}$.

Instrument type, method of analysis and source of data are listed alphabetically by stations in Table 3 . If UMINOR < 0 , ellipse rotates clockwise
$10.8+-0.1 \quad 63+-\quad 3 \quad 9.8+-0.4 \quad 342+-4$ $6.0+-0.1 \quad 30+-7 \quad 5.2+-0.1 \quad 305+-5$

$5.4+-0.8 \quad 71+-8 \quad 5.6+-1.0 \quad 341+-10$

$4.9+-0.6 \quad 72+-7 \quad 5.1+-0.8 \quad 343+-9$

$1.6+-0.6 \quad 59+-20 \quad 0.4+-0.4 \quad 308+-54$

$0.7+-0.2 \quad 74+-19 \quad 0.1+-0.1 \quad 41+-49$

$8.6+-0.3 \quad 67+-\quad 4 \quad 8.3+-0.3 \quad 340+-5$

$7.9+-0.4 \quad 59+-6 \quad 7.6+-0.4 \quad 331+-6$

10.7

63

27.6

300

8.9

253

$19.7 \quad 318$

$181 \quad 145 \quad 35$

$11.2+-0.0$

$-9.4+-0.5$

$38+-6$

$61+-3$

5.6

5.1

$-5 \cdot 4$
$-4 \cdot 9$

341

354

0
12

1.6

0.7

$-0.4$

60

74

83

8.7

8.0

$-8 \cdot 2$

41

39

62

69

23.4

$-8.7$

294

343

20.1

$-7.9$

313

77

0.9

$-0.3$

97

45 
Table 5. $M_{2}$ tidal current parameters-Continued

Table 5. M2 TLDAL CURRENT PARAMETERS--Continued

\begin{tabular}{|c|c|c|}
\hline STATION & RECORD INSTR & ABOVE \\
\hline $\begin{array}{l}\text { LAT. } \\
\text { LONG. }\end{array}$ & $\begin{array}{l}\text { LENGTH DEPTH } \\
\text { (DAYS) }\end{array}$ & $\begin{array}{l}\text { 1 ВОTTOM } \\
\text { (M) }\end{array}$ \\
\hline
\end{tabular}

$\begin{array}{cccr} & \text { FOURIER } & \text { COEFFICIENTS } & \\ \text { EAST } & \text { PHASE } & \text { NORTH } & \text { PHASE } \\ (\mathrm{CM} / \mathrm{SEC}) & (\mathrm{DEG}-\mathrm{G}) & (\mathrm{CM} / \mathrm{SEC}) & (\mathrm{DEG}-\mathrm{G})\end{array}$

$\begin{array}{cccc}\text { CURRENT } & \text { ELLIPSE } & \text { PARAMETERS } \\ \text { UMAJOR } & \text { UMINOR } & \text { PHASE } & \text { ORIEN } \\ (\text { CM/SEC }) & (\text { CM/SEC }) & \text { (DEG-G) } & \text { (DEG-TRUE) }\end{array}$

NEW ENGLAND SHELF--CONT.

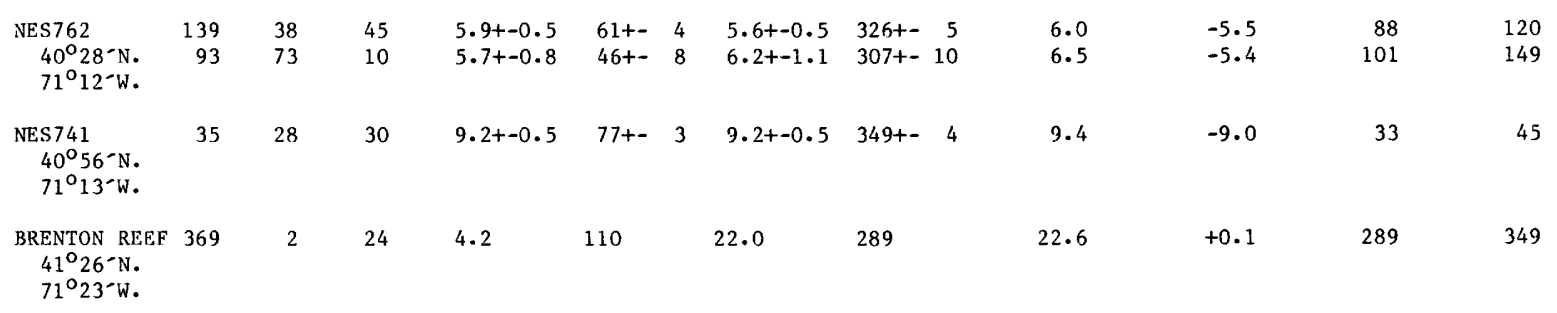

MIDDLE ATLANTIC BIGHT

\begin{tabular}{|c|c|c|c|c|c|c|c|c|c|c|c|}
\hline $\begin{array}{l}\text { NES } 763 \mathrm{~W} \\
39^{\circ} 43^{-} \mathrm{N} . \\
71^{\circ} 47^{-} \mathrm{W} .\end{array}$ & 183 & 302 & 202 & $1.7+-0.6$ & $66+-99$ & $1.2+-0.5$ & $285+-24$ & 2.0 & -0.6 & 258 & 303 \\
\hline $\begin{array}{l}\text { P.32 } \\
\quad 40^{\circ} 15^{-} \mathrm{N} . \\
71^{\circ} 51^{-\mathrm{W}} .\end{array}$ & 62 & 70 & 5 & $4 \cdot 5$ & 25 & 3.9 & 274 & 5.0 & -3.3 & 230 & 303 \\
\hline $\begin{array}{l}\text { LI } 4 \\
40^{\circ} 01-\mathrm{N} . \\
71^{\circ} 53^{-} \mathrm{W} .\end{array}$ & 56 & 76 & 15 & 7.0 & 42 & 6.9 & 287 & 8.3 & $-5 \cdot 3$ & 253 & 316 \\
\hline $\begin{array}{l}\text { NES } 762 \mathrm{~W} \\
39^{\circ} 55^{-} \mathrm{N} . \\
71^{\circ} 58^{-} \mathrm{W} .\end{array}$ & 172 & 38 & 45 & $7.4+-0.5$ & $48+-\quad 4$ & $6.8+-0.6$ & $290+-5$ & 8.6 & $-5 \cdot 1$ & 255 & 310 \\
\hline $\begin{array}{l}\text { LI3 } \\
\qquad 40^{\circ} 11^{-} \mathrm{N} . \\
72^{\circ} 00^{-} \mathrm{W}\end{array}$ & 168 & 3 & 64 & 7.9 & 49 & 6.1 & 298 & 8.5 & $-5 \cdot 3$ & 247 & 297 \\
\hline
\end{tabular}

Instrument type, method of analysis and source of data are 1isted alphabetically by stations in Table 3 .

If UMINOR < 0, ellipse rotates clockwise

$\mathrm{H}$ - harmonic analysis

$\mathrm{R}$ - response analysis 
Table 5. $M_{2}$ tidal current parameters-Continued

Table 5. M2 TIDAL CURRENT PARAMETERS---Continued

\begin{tabular}{|c|c|c|c|c|c|c|c|c|c|c|c|}
\hline STATION & RECORD & INSTR & ABOVE & & OURIER & COEFFICIENTS & & & ELLIPSE & PARAMETERS & \\
\hline $\begin{array}{l}\text { LAT. } \\
\text { LONG. }\end{array}$ & $\begin{array}{l}\text { LENGTH } \\
\text { (DAYS) }\end{array}$ & $\begin{array}{l}\text { DEPTH } \\
\text { (M) }\end{array}$ & $\begin{array}{l}\text { воттом } \\
\text { (M) }\end{array}$ & $\begin{array}{c}\text { EAST } \\
(\mathrm{CM} / \mathrm{SEC})\end{array}$ & $\begin{array}{c}\text { PHASE } \\
(\mathrm{DEG}-\mathrm{G})\end{array}$ & $\begin{array}{c}\text { NORTH } \\
(\mathrm{CM} / \mathrm{SEC})\end{array}$ & $\begin{array}{r}\text { PHASE } \\
(D E G-G)\end{array}$ & $\begin{array}{r}\text { UMAJOR } \\
\text { (CM/SEC) }\end{array}$ & $\begin{array}{c}\text { UMINOR } \\
(\mathrm{CM} / \mathrm{SEC})\end{array}$ & $\begin{array}{r}\text { PHASE } \\
(\text { DEG-G) }\end{array}$ & $\begin{array}{c}\text { ORIEN } \\
\text { (DEG-TRUE) }\end{array}$ \\
\hline
\end{tabular}

MIDDLE ATLANTIC BIGHT--CONT.

\begin{tabular}{|c|c|c|c|c|c|c|c|c|c|c|c|c|}
\hline $\begin{array}{l}\text { LI2 } \\
\qquad 40^{\circ} 25^{-\mathrm{N}} \\
\quad 72^{\circ} 08^{-} \mathrm{W}\end{array}$ & 283 & 3 & 56 & 8.6 & 54 & & 4.9 & 309 & 8.7 & -4.7 & 240 & 282 \\
\hline $\begin{array}{l}\text { P31 } \\
\quad 40^{\circ} 39-\mathrm{N} . \\
72^{\circ} 15^{-W} .\end{array}$ & 67 & 42 & 5 & 7.2 & 43 & & 1.6 & 310 & 7.2 & $-1 \cdot 6$ & 223 & 271 \\
\hline $\begin{array}{l}\text { LT4 } \\
\quad 40^{\circ} 34^{-} \mathrm{N} . \\
72^{\circ} 19^{-} \mathrm{W} .\end{array}$ & $\begin{array}{r}29 \\
203 \\
174 \\
87\end{array}$ & $\begin{array}{r}3 \\
24 \\
44 \\
51\end{array}$ & $\begin{array}{r}49 \\
28 \\
8 \\
1\end{array}$ & $\begin{array}{r}9.5+-2.0 \\
15.7+-2.5 \\
11.1+-0.9 \\
5.0+-0.3\end{array}$ & $\begin{array}{l}67+- \\
57+- \\
62+- \\
51+-\end{array}$ & $\begin{array}{r}3 \\
19 \\
6 \\
5\end{array}$ & $\begin{array}{l}4 \cdot 0+-1 \cdot 9 \\
6.7+-1 \cdot 5 \\
4 \cdot 3+-1 \cdot 1 \\
1.3+-0.1\end{array}$ & $\begin{array}{rr}329+- & 25 \\
323+- & 22 \\
321+- & 14 \\
298+- & 8\end{array}$ & $\begin{array}{r}9.6+-2.0 \\
15.8+-2.7 \\
11.2+-1.0 \\
5.0+-0.3\end{array}$ & $\begin{array}{l}-3 \cdot 9+-2 \cdot 0 \\
-6 \cdot 4+-1 \cdot 2 \\
-4 \cdot 1+-1 \cdot 0 \\
-1 \cdot 1+-0.1\end{array}$ & $\begin{array}{lr}247+- & 0 \\
237+- & 20 \\
244+- & 5 \\
232+- & 5\end{array}$ & $\begin{array}{l}272+- \\
272+- \\
275+- \\
276+-\end{array}$ \\
\hline $\begin{array}{l}\text { LI } 1 \\
\qquad \begin{array}{l}40^{\circ} 34-\mathrm{N} . \\
72^{\circ} 19^{-} \mathrm{W} .\end{array}\end{array}$ & 60 & 33 & 15 & 7.7 & 73 & & 2.8 & 349 & 7.7 & -2.8 & 252 & 267 \\
\hline $\begin{array}{l}\text { CMICE } \\
40^{\circ} 47^{-} \mathrm{N} . \\
72^{\circ} 29^{-} \mathrm{W} .\end{array}$ & $\begin{array}{l}25 \\
25 \\
25 \\
25\end{array}$ & $\begin{array}{r}4 \\
8 \\
16 \\
25\end{array}$ & $\begin{array}{r}25 \\
21 \\
13 \\
4\end{array}$ & $\begin{array}{r}10.2+-1.4 \\
10.4+-1.1 \\
9.2+-0.8 \\
6.6+-0.7\end{array}$ & $\begin{array}{l}62+- \\
60+- \\
57+- \\
39+-\end{array}$ & $\begin{array}{l}8 \\
6 \\
5 \\
6\end{array}$ & $\begin{array}{l}1.6+-0.9 \\
2.5+-1.3 \\
3.1+-0.8 \\
2.6+-1.2\end{array}$ & $\begin{array}{l}314+-31 \\
288+-29 \\
270+-14 \\
219+-27\end{array}$ & $\begin{array}{r}10.2 \\
10.5 \\
9.6 \\
7.1\end{array}$ & $\begin{array}{l}-1.5 \\
-1.8 \\
-1.6 \\
+0.0\end{array}$ & $\begin{array}{l}242 \\
242 \\
240 \\
219\end{array}$ & $\begin{array}{l}273 \\
279 \\
286 \\
292\end{array}$ \\
\hline $\begin{array}{l}\mathrm{ME} \\
39^{\circ} 57^{-\mathrm{N}} . \\
72^{\circ} 36^{-} \mathrm{W} .\end{array}$ & 29 & 59 & 1 & 4.6 & 24 & & 6.1 & 250 & 7.1 & -2.9 & 235 & 326 \\
\hline $\begin{array}{l}30 \\
40^{\circ} 03^{-} \mathrm{N} . \\
72^{\circ} 42^{-} \mathrm{W} .\end{array}$ & 107 & 42 & 17 & 13.3 & 50 & & 11.3 & 287 & 15.4 & $-8 \cdot 2$ & 251 & 307 \\
\hline $\begin{array}{l}\mathrm{LTM}^{* *} \\
\qquad \begin{array}{l}0^{\circ} 07^{-\mathrm{N}} \\
72^{\circ} 55^{-\mathrm{W}} .\end{array}\end{array}$ & $\begin{array}{l}120 \\
120\end{array}$ & $\begin{array}{r}3 \\
39\end{array}$ & $\begin{array}{r}44 \\
8\end{array}$ & $\begin{array}{r}10.9+-0.8 \\
9.8+-0.3\end{array}$ & $\begin{array}{l}114+- \\
119+-\end{array}$ & $\begin{array}{r}0 \\
15\end{array}$ & $\begin{array}{l}7.8+-1.4 \\
7.2+-0.3\end{array}$ & $\begin{array}{l}244+-6 \\
259+-10\end{array}$ & $\begin{array}{l}12 \cdot 3+-1.5 \\
11.5+-0.5\end{array}$ & $\begin{array}{l}+5.2+-0.3 \\
+3.9+-0.3\end{array}$ & $\begin{array}{l}279+-2 \\
286+-14\end{array}$ & $\begin{array}{l}301+- \\
304+-\end{array}$ \\
\hline $\begin{array}{l}\text { NJ4 } \\
38^{\circ} 55^{\circ} \mathrm{N} . \\
72^{\circ} 58^{-} \mathrm{W} .\end{array}$ & $\begin{array}{l}72 \\
72 \\
72\end{array}$ & $\begin{array}{r}3 \\
43 \\
91\end{array}$ & $\begin{array}{r}89 \\
49 \\
1\end{array}$ & $\begin{array}{r}8.4+-2.1 \\
11.9+-1.5 \\
1.0+-0.4\end{array}$ & $\begin{array}{l}55+- \\
66+- \\
34+-\end{array}$ & $\begin{array}{r}14 \\
7 \\
24\end{array}$ & $\begin{array}{l}6.2+-1.7 \\
9.9+-1.9 \\
0.7+-0.4\end{array}$ & $\begin{array}{r}308+-16 \\
302+-11 \\
97+-33\end{array}$ & $\begin{array}{r}8.8 \\
13.8 \\
1.1\end{array}$ & $\begin{array}{l}-5.7 \\
-7.1 \\
+0.6\end{array}$ & $\begin{array}{l}250 \\
267 \\
229\end{array}$ & $\begin{array}{l}292 \\
306 \\
244\end{array}$ \\
\hline $\begin{array}{l}\text { MA } \\
39^{\circ} 27^{-} \mathrm{N} . \\
73^{\circ} 00^{-} \mathrm{w} .\end{array}$ & 58 & 58 & 1 & $5.4+-0.2$ & $17+-$ & 5 & $6.0+-1.5$ & $248+-$ & $7 \cdot 3+-1.3$ & $-3.4+-0.1$ & $226+-9$ & $319+-$ \\
\hline $\begin{array}{l}\text { LT3 } \\
\quad 39^{\circ} 16^{-} \mathrm{N} . \\
73^{\circ} 02^{-} \mathrm{W} .\end{array}$ & $\begin{array}{r}154 \\
104 \\
104 \\
70\end{array}$ & $\begin{array}{r}3 \\
9 \\
19 \\
58\end{array}$ & $\begin{array}{l}67 \\
61 \\
51 \\
12\end{array}$ & $\begin{array}{l}10.9 \\
13.9+-1.2 \\
16.1+-1.6 \\
10.7+-1.1\end{array}$ & $\begin{array}{l}70 \\
69+- \\
66+- \\
80+-\end{array}$ & $\begin{array}{l}5 \\
6 \\
6\end{array}$ & $\begin{array}{r}9 \cdot 2 \\
10 \cdot 2+-1 \cdot 0 \\
12.7+-1 \cdot 3 \\
8.9+-1.1\end{array}$ & $\begin{array}{ll}308 & \\
307+- & 6 \\
305+- & 6 \\
319+- & 7\end{array}$ & $\begin{array}{l}12.6 \\
15.4 \\
18.1 \\
12.2\end{array}$ & $\begin{array}{l}-6.8 \\
-7.8 \\
-9.7 \\
-6.7\end{array}$ & $\begin{array}{l}272 \\
265 \\
265 \\
281\end{array}$ & $\begin{array}{l}306 \\
300 \\
303 \\
305\end{array}$ \\
\hline
\end{tabular}

Instrument type, method of analysis and source of data are listed alphabetically by stations in Table 3. If UMINOR < 0 , ellipse rotates clockwise

** average of 2-60 day records 
Table 5. $M_{2}$ tidal current parameters-Continued

Table 5. M2 TIDAL CURRENT PARAMETERS-Continued

\begin{tabular}{|c|c|c|c|c|c|c|c|c|c|}
\hline STATION & RECORD INSTR & ABOVE & & JRIER & COEFFICIENTS & & $* *$ & ENT ELLIPSE & PARAMETERS \\
\hline $\begin{array}{c}\text { LAT. } \\
(\mathrm{CM} / \mathrm{SEC})\end{array}$ & $\begin{array}{l}\text { LENGTH DEPTH } \\
(\mathrm{CM} / \mathrm{SEC})\end{array}$ & $\begin{array}{l}\text { ВОTTOM } \\
\text { (DEG-G) }\end{array}$ & $\begin{array}{c}\text { EAST } \\
\text { (DEG-TRUE) }\end{array}$ & PHASE & NORTH & PHASE & UMAJOR & UMINOR & PHASE ORIEN \\
\hline
\end{tabular}

MIDDLE ATLANTIC BIGHT--CONT.

\begin{tabular}{|c|c|c|c|c|c|c|c|c|c|c|c|c|c|}
\hline $\begin{array}{l}\text { LT7 } \\
\quad 39^{\circ} 55^{-N} \text {. } \\
73^{\circ} 05^{-W} \text {. }\end{array}$ & $\begin{array}{r}84 \\
135\end{array}$ & $\begin{array}{r}3 \\
58\end{array}$ & $\begin{array}{r}63 \\
8\end{array}$ & $\begin{array}{r}10.2 \\
8.3\end{array}$ & $\begin{array}{l}77 \\
90\end{array}$ & & $\begin{array}{l}7.9 \\
7.4\end{array}$ & $\begin{array}{l}296 \\
309\end{array}$ & & $\begin{array}{l}12.2 \\
10.5\end{array}$ & $\begin{array}{l}-4.1 \\
-3.7\end{array}$ & $\begin{array}{l}271 \\
287\end{array}$ & $\begin{array}{l}306 \\
311\end{array}$ \\
\hline $\begin{array}{l}\text { MESA7 } \\
39^{\circ} 55^{-} \mathrm{N} . \\
73^{\circ} 06^{-} \mathrm{W} .\end{array}$ & $\begin{array}{r}107 \\
103 \\
63\end{array}$ & $\begin{array}{l}18 \\
38 \\
66\end{array}$ & $\begin{array}{r}50 a \\
30 a \\
2 a\end{array}$ & $\begin{array}{r}17.5+-1.9 \\
15.1+-1.5 \\
4.2+-0.8\end{array}$ & $\begin{array}{l}74+- \\
67+- \\
84+-\end{array}$ & $\begin{array}{r}6 \\
5 \\
12\end{array}$ & $\begin{array}{r}10.1+-1.4 \\
9.9+-1.4 \\
5.0+-0.7\end{array}$ & $\begin{array}{l}300+- \\
295+- \\
285+-\end{array}$ & $\begin{array}{l}8 \\
8 \\
8\end{array}$ & $\begin{array}{r}19.1 \\
16.8 \\
6.4\end{array}$ & $\begin{array}{l}-6.7 \\
-6.6 \\
-1.2\end{array}$ & $\begin{array}{l}263 \\
259 \\
276\end{array}$ & $\begin{array}{l}295 \\
299 \\
320\end{array}$ \\
\hline $\begin{array}{l}\text { FIRE IS. } \\
40^{\circ} 29^{\circ} \mathrm{N} . \\
73^{\circ} 11^{-} \mathrm{W} .\end{array}$ & 369 & 2 & 27 & 6.7 & 91 & & 1.0 & 312 & & 6.7 & -0.6 & 271 & 276 \\
\hline $\begin{array}{l}\text { P12 } \\
39^{\circ} 09^{-N} \text {. } \\
73^{\circ} 13^{-W} \text {. }\end{array}$ & 115 & 57 & 5 & 8.0 & 53 & & 7.4 & 290 & & 9.6 & -5.1 & 258 & 311 \\
\hline $\begin{array}{l}28 \\
40^{\circ} 16^{-} \mathrm{N} . \\
73^{\circ} 13^{-} \mathrm{W} .\end{array}$ & 111 & 3 & 35 & 10.8 & 83 & & 5.9 & 296 & & 12.0 & -2.9 & 270 & 297 \\
\hline $\begin{array}{l}\text { NJ3 } \\
39^{\circ} 04^{-} \mathrm{N} . \\
73^{\circ} 20^{-} \mathrm{W} .\end{array}$ & 1LL & 47 & 15 & 11.1 & 62 & & 10.5 & 304 & & 13.1 & -7.9 & 270 & 312 \\
\hline $\begin{array}{l}15 \\
40^{\circ} 26^{-} \mathrm{N} \\
73^{\circ} 28^{-} \mathrm{W}\end{array}$ & 59 & 3 & 20 & 12.0 & 86 & & 4.0 & 284 & & 12.6 & -1.2 & 267 & 288 \\
\hline $\begin{array}{l}\mathrm{MC} \\
38^{\circ} 33^{-} \mathrm{N} \\
73^{\circ} 31^{-W}\end{array}$ & 29 & 79 & 1 & 4.9 & 41 & & 5.5 & 271 & & 6.7 & -3.1 & 250 & 321 \\
\hline $\begin{array}{l}49 \\
39^{\circ} 38^{-} \mathrm{N} \\
73^{\circ} 34^{-} \mathrm{W}\end{array}$ & 111 & 27 & 8 & 9.9 & 49 & & 9.2 & 284 & & 12.1 & $-6 \cdot 2$ & 253 & 319 \\
\hline
\end{tabular}

Instrument type, method of analysis and source of data are listed alphabetically by stations in Tables 3.

If UMINOR < 0 , ellipse rotates clockwise

a- water depth taken from chart 13200 
Table 5. $M_{2}$ tidal current parameters-Continued

Table 5. M2 TIDAL CURRENT PARAMETERS--Continued

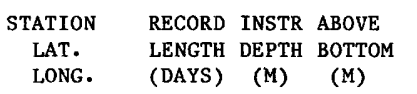

$\begin{array}{cccc} & \text { FOURIER } & \text { COEFFICIENTS } & \\ \text { EAST } & \text { PHASE } & \text { NORTH } & \text { PHASE } \\ (\mathrm{CM} / \mathrm{SEC}) & (\mathrm{DEG}-\mathrm{G}) & (\mathrm{CM} / \mathrm{SEC}) & (\mathrm{DEG}-\mathrm{G})\end{array}$

\begin{tabular}{ccccc}
\multicolumn{2}{c}{ CURRENT } & ELLIPSE & \multicolumn{2}{c}{ PARAMETERS } \\
UMAJOR & UMINOR & PHASE & ORIEN \\
$(\mathrm{CM} / \mathrm{SEC})$ & $(\mathrm{CM} / \mathrm{SEC})$ & (DEG-G) & (DEG-TRUE)
\end{tabular}

MIDDLE ATLANTIC BIGHT--CONT.

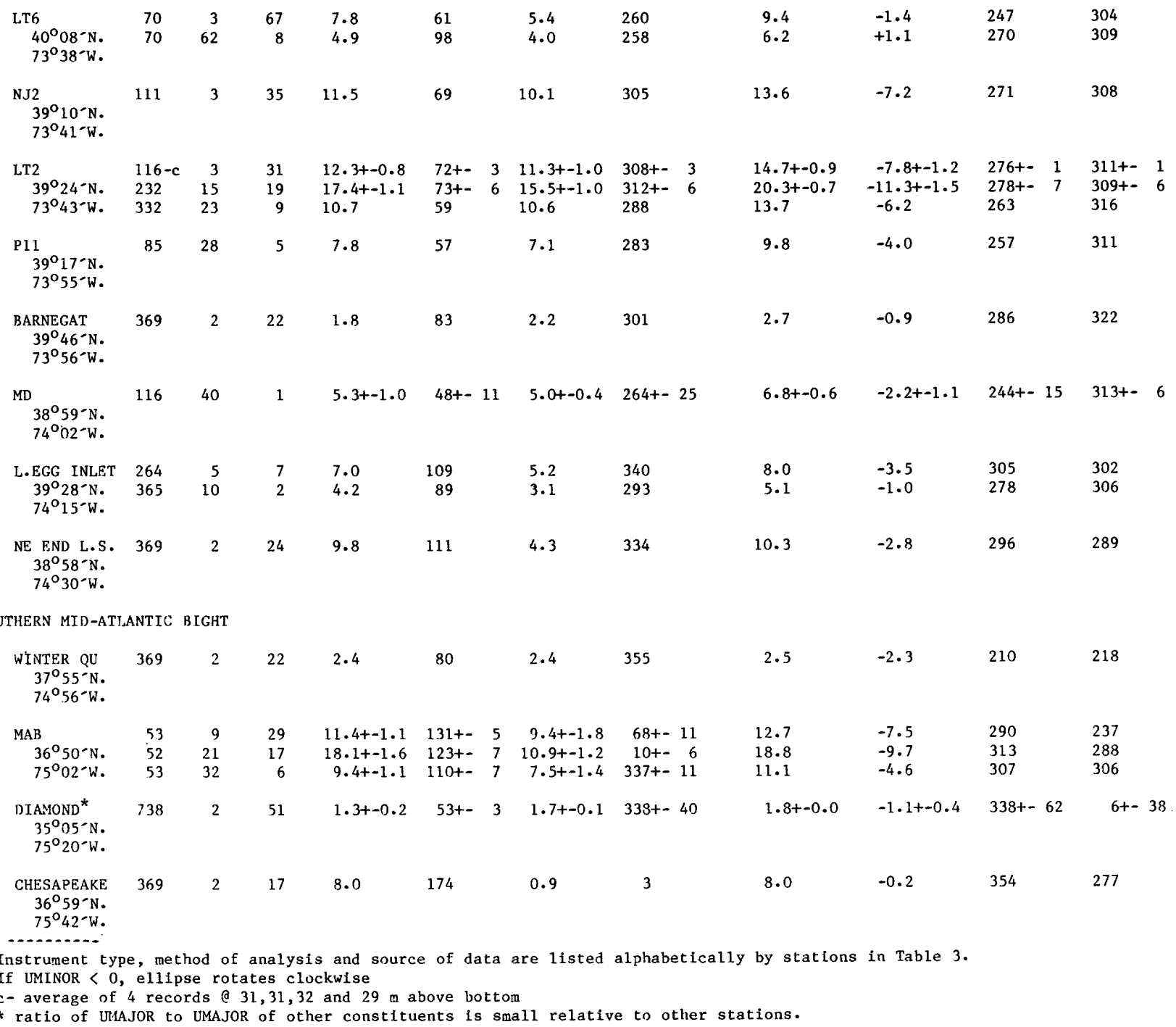


Table 5. $M_{2}$ tidal current parameters-Continued

Table 5. M2 TIDAL CURRENT PARAMETERS--Continued

STATION RECORD INSTR ABOVE

LAT. LENGTH DEPTH BOTTOM

LONG.

(DAYS) (M)

$\begin{array}{cc} & \text { FOURIER } \\ \text { EAST } & \text { PHASE } \\ (\mathrm{CM} / \mathrm{SEC}) & (\mathrm{DEG}-\mathrm{G})\end{array}$

COEFF ICIENTS
NORTH
$(\mathrm{CM} / \mathrm{SEC})$

OCEANIC

$\begin{array}{lrrr}\text { S8 } & 415 & 70 & 2470 \\ 42^{\circ} 00^{-N} . & 415 & 1500 & 1040\end{array}$ $63^{\circ} 30^{-}$W. $\quad 96 \quad 2530 \quad r$

SITE $D^{*} \quad 104 \quad 14 \quad 2736$

$39^{\circ} 20^{\prime} \mathrm{N} . \quad 87 \quad 104 \quad 2646$

$69^{\circ} 57^{-W}$ W. $153 \quad 1000 \quad 1750$

$\begin{array}{llr}92 & 2061 & 689\end{array}$

$43 \quad 2572 \quad 178$

NES765 $39^{\circ} 17^{-N}$. $70^{\circ} 50^{-} \mathrm{W}$.

$180 \quad 1995 \quad 655$

$$
\text { . }
$$

$7.1+-2.2 \quad 101+-26 \quad 5.9+-2.1 \quad 325+-16$

$\begin{array}{llll}0.8+-0.6 & 185+-103 & 0.7+-0.7 & 218+-67\end{array}$

0.6

185

0.8

229

$3.5+-0.6 \quad 118+-20 \quad 2.3+-0.5 \quad 44+-21$

$2.4+-0.0 \quad 115+-44 \quad 1.5+-0.4 \quad 19+-52$

$1.0+-0.1 \quad 90+-21 \quad 0.4+-0.1 \quad 345+-63$

$1.1+-0.2 \quad 132+-11 \quad 0.4+-0.2 \quad 85+-22$

$1.1+-0.2 \quad 351+-20 \quad 0.6+-0.2 \quad 268+-46$

$0.6+-0.2 \quad 49+-19 \quad 0.4+-0.2 \quad 240+-24$

Instrument type, method of analysis and source of data are listed alphabetically by stations in Table 3 .

If UMINOR < 0 , ellipse rotates clockwise

* - Only records with values for both east and north were chosen. Where there was only 1 record (depths 14 \& $2572 \mathrm{~m}$ ) the error bars are taken from Regal and Wunsch,1973. 
Table 6. $\mathrm{N}_{2}$ tidal current parameters (see table 5 for explanation).

Table 6. N2 TIDAL CURRENT PARAMETERS

\begin{tabular}{|c|c|c|c|c|c|c|}
\hline IATION & ORD & INSTR & ABOVE & & UUR IER & COEFFICIENTS \\
\hline $\begin{array}{l}\text { LAT. } \\
\text { LONG. }\end{array}$ & $\begin{array}{l}\text { LENGTH } \\
\text { (DAYS) }\end{array}$ & $\begin{array}{l}\text { DEPTH } \\
\text { (M) }\end{array}$ & $\begin{array}{l}\text { BOTTOM } \\
\text { (M) }\end{array}$ & $\begin{array}{c}\text { EAST } \\
(\mathrm{CM} / \mathrm{SEC})\end{array}$ & $\begin{array}{l}\text { PHASE } \\
(\text { DEG -G) }\end{array}$ & $\begin{array}{c}\text { NORTH } \\
(\mathrm{CM} / \mathrm{SEC})\end{array}$ \\
\hline
\end{tabular}

\begin{tabular}{cccc}
\multicolumn{2}{c}{ CURRENT ELLIPSE } & PARAMETERS \\
UMAJOR & UMINOR & PHASE & ORIEN \\
$(\mathrm{CM} / \mathrm{SEC})$ & $(\mathrm{CM} / \mathrm{SEC})$ & (DEG-G) & (DEG-TRUE)
\end{tabular}

SCOTIAN SHELF

\begin{tabular}{|c|c|c|c|c|c|c|c|c|c|c|c|}
\hline $\begin{array}{l}\mathrm{SS} 3 \\
\quad 43^{\circ} 22^{\circ} \mathrm{N} . \\
62^{\circ} 40^{-} \mathrm{W} .\end{array}$ & $\begin{array}{r}135 \\
109 \\
47 \\
121\end{array}$ & $\begin{array}{l}20 \\
50 \\
81 \\
95\end{array}$ & $\begin{array}{r}79 \\
49 \\
18 \\
4\end{array}$ & $\begin{array}{l}3.8+-0.3 \\
3.1+-0.5 \\
2.5 \\
1.6+-0.8\end{array}$ & $\begin{array}{l}42+-26 \\
19+-22 \\
37 \\
357+-32\end{array}$ & $\begin{array}{l}3.5+-0.9 \\
4.1+-1.0 \\
2.8 \\
2.4+-0.4\end{array}$ & $\begin{array}{l}280+-21 \\
263+-11 \\
273 \\
254+-22\end{array}$ & $\begin{array}{l}4.5+-0.5 \\
4.5+-1.2 \\
3.3 \\
2.5+-0.5\end{array}$ & $\begin{array}{l}-2.5+-0.6 \\
-2.6+-0.1 \\
-1.7 \\
-1.5+-0.6\end{array}$ & $\begin{array}{l}249+-20 \\
245+-15 \\
250 \\
241+-7\end{array}$ & $\begin{array}{lr}313+- & 12 \\
331+- & 1 \\
321 & \\
342+- & 20\end{array}$ \\
\hline $\begin{array}{l}\text { SS7 } \\
\quad 43^{\circ} 02^{-} \mathrm{N} . \\
62^{\circ} 54^{-} \mathrm{W} .\end{array}$ & $\begin{array}{l}27 \\
27\end{array}$ & $\begin{array}{r}50 \\
118\end{array}$ & $\begin{array}{r}75 \\
7\end{array}$ & $\begin{array}{l}1.1 \\
1.9\end{array}$ & $\begin{array}{l}56 \\
26\end{array}$ & $\begin{array}{l}1.9 \\
2.5\end{array}$ & $\begin{array}{l}254 \\
259\end{array}$ & $\begin{array}{l}2.2 \\
2.8\end{array}$ & $\begin{array}{l}-0.3 \\
-1.3\end{array}$ & $\begin{array}{l}250 \\
242\end{array}$ & $\begin{array}{l}331 \\
327\end{array}$ \\
\hline $\begin{array}{l}\text { SS6 } \\
\quad 43^{\circ} 15^{-} \mathrm{N} \\
63^{\circ} 22^{-} \mathrm{W}\end{array}$ & $\begin{array}{l}26 \\
27\end{array}$ & $\begin{array}{r}50 \\
130\end{array}$ & $\begin{array}{r}85 \\
5\end{array}$ & $\begin{array}{l}2.0 \\
0.8\end{array}$ & $\begin{array}{r}61 \\
149\end{array}$ & $\begin{array}{l}1.5 \\
1.2\end{array}$ & $\begin{array}{r}273 \\
5\end{array}$ & $\begin{array}{l}2.4 \\
1.4\end{array}$ & $\begin{array}{l}-0.7 \\
-0.4\end{array}$ & $\begin{array}{l}252 \\
355\end{array}$ & $\begin{array}{l}306 \\
329\end{array}$ \\
\hline $\begin{array}{l}\text { S5 } \\
\quad \begin{array}{l}42^{\circ} 30^{-} \mathrm{N} \\
63^{\circ} 30^{-} \mathrm{N}\end{array}\end{array}$ & $\begin{array}{l}262 \\
262 \\
370\end{array}$ & $\begin{array}{r}50 \\
150 \\
1534\end{array}$ & $\begin{array}{r}1470 \\
1370 \\
10\end{array}$ & $\begin{array}{l}2.3+-2.6 \\
1.3+-1.5 \\
0.7\end{array}$ & $\begin{array}{l}170+-4 \\
220+-120 \\
48\end{array}$ & $\begin{array}{l}2.7+-3.0 \\
1.3+-1.1 \\
0.8\end{array}$ & $\begin{array}{l}127+-62 \\
146+-102 \\
265\end{array}$ & $\begin{array}{l}2.8+-2.9 \\
1.6+-1.5 \\
1.0\end{array}$ & $\begin{array}{l}-2.0+-2.8 \\
-0.9+-1.0 \\
-0.3\end{array}$ & $\begin{array}{l}134+-52 \\
164+-77 \\
249\end{array}$ & $\begin{array}{l}25+-12 \\
26+-35 \\
320\end{array}$ \\
\hline $\begin{array}{l}\text { SS } 4 \\
\quad 42^{\circ} 40^{-} \mathrm{N} . \\
63^{\circ} 30^{-} \mathrm{W} .\end{array}$ & $\begin{array}{l}585 \\
204 \\
584 \\
363 \\
210 \\
296\end{array}$ & $\begin{array}{r}50 \\
100 \\
150 \\
500 \\
690 \\
990\end{array}$ & $\begin{array}{r}970 \\
920 \\
870 \\
520 \\
330 \\
30\end{array}$ & $\begin{array}{l}0.8+-0.8 \\
0.7 \\
0.9+-0.6 \\
0.7 \\
1.3+-0.1 \\
1.1+-0.3\end{array}$ & $\begin{array}{l}262+-120 \\
148 \\
126+-94 \\
117 \\
19+-17 \\
81+-53\end{array}$ & $\begin{array}{l}0.6+-0.1 \\
0.9 \\
0.9+-0.0 \\
0.7 \\
1.9+-0.2 \\
1.1+-0.6\end{array}$ & $\begin{array}{l}351+-85 \\
18 \\
38+-115 \\
343 \\
253+-5 \\
311+-109\end{array}$ & $\begin{array}{l}0.9+-0.6 \\
1.0 \\
1.1+-0.3 \\
0.9 \\
2.1+-0.3 \\
1.4+-0.6\end{array}$ & $\begin{array}{l}-0.3+-0.3 \\
-0.5 \\
-0.7+-0.2 \\
-0.4 \\
-0.9+-0.1 \\
-0.6+-0.5\end{array}$ & $\begin{array}{l}331+-49 \\
1 \\
246+-93 \\
322 \\
240+-7 \\
245+-98\end{array}$ & $\begin{array}{l}332+-59 \\
326 \\
31+-57 \\
318 \\
332+-\quad 3 \\
359+-54\end{array}$ \\
\hline $\begin{array}{l}\mathrm{S} 3 \\
42^{\circ} 45^{-} \mathrm{N} . \\
63^{\circ} 30^{-} \mathrm{W}\end{array}$ & $\begin{array}{r}371 \\
97 \\
262\end{array}$ & $\begin{array}{l}230 \\
500 \\
690\end{array}$ & $\begin{array}{r}480 \\
210 \\
20\end{array}$ & $\begin{array}{l}0.4 \\
0.9 \\
0.9\end{array}$ & $\begin{array}{r}70 \\
152 \\
110\end{array}$ & $\begin{array}{l}0.9 \\
1.3 \\
0.4\end{array}$ & $\begin{array}{r}280 \\
103 \\
37\end{array}$ & $\begin{array}{l}1.0 \\
1.4 \\
0.9\end{array}$ & $\begin{array}{l}-0.2 \\
-0.6 \\
-0.3\end{array}$ & $\begin{array}{l}276 \\
116 \\
107\end{array}$ & $\begin{array}{r}341 \\
30 \\
83\end{array}$ \\
\hline $\begin{array}{l}\mathrm{S} 1 \\
42^{\circ} 49^{-} \mathrm{N} . \\
63^{\circ} 30^{-} \mathrm{W} .\end{array}$ & $\begin{array}{l}266 \\
752 \\
180 \\
632 \\
572\end{array}$ & $\begin{array}{r}20 \\
50 \\
100 \\
150 \\
230\end{array}$ & $\begin{array}{r}220 \\
190 \\
140 \\
90 \\
10\end{array}$ & $\begin{array}{l}2.4+-1.2 \\
1.9+-0.7 \\
1.0 \\
1.4+-0.6 \\
1.4+-0.4\end{array}$ & $\begin{array}{cc}356+- & 84 \\
28+- & 14 \\
84 & \\
47+- & 1 \\
33+- & 3\end{array}$ & $\begin{array}{l}4.2+-1.7 \\
2.6+-1.4 \\
1.8 \\
1.8+-0.5 \\
1.6+-0.4\end{array}$ & $\begin{array}{l}247+-57 \\
273+-5 \\
326 \\
286+-13 \\
288+-10\end{array}$ & $\begin{array}{l}4.5+-2.0 \\
2.9+-1.3 \\
1.9 \\
2.0+-0.8 \\
1.7+-0.5\end{array}$ & $\begin{array}{l}-1.8+-0.7 \\
-1.5+-0.6 \\
-0.8 \\
-1.0+-0.2 \\
-1.2+-0.2\end{array}$ & $\begin{array}{l}248+-50 \\
254+-13 \\
318 \\
268+-18 \\
267+-13\end{array}$ & $\begin{array}{lr}357+- & 26 \\
330+- & 24 \\
341 & \\
328+- & 12 \\
332+- & 6\end{array}$ \\
\hline $\begin{array}{l}\mathrm{S} 6 \\
43^{\circ} 00^{-} \mathrm{N} . \\
63^{\circ} 30^{-} \mathrm{W} .\end{array}$ & $\begin{array}{l}250 \\
492 \\
182 \\
380\end{array}$ & $\begin{array}{r}20 \\
50 \\
100 \\
153\end{array}$ & $\begin{array}{r}150 \\
120 \\
70 \\
17\end{array}$ & $\begin{array}{l}3 \cdot 1 \\
1.1+-0.2 \\
1.3 \\
1.3+-1.2\end{array}$ & $\begin{array}{l}357 \\
35+-22 \\
72 \\
13+-21\end{array}$ & $\begin{array}{l}4 \cdot 6 \\
2 \cdot 3+-0.3 \\
1.9 \\
2 \cdot 4+-1.4\end{array}$ & $\begin{array}{l}250 \\
287+-22 \\
318 \\
266+-19\end{array}$ & $\begin{array}{l}4.7 \\
2.3+-0.3 \\
2.0 \\
2.4+-1.5\end{array}$ & $\begin{array}{l}-2 \cdot 9 \\
-1 \cdot 1+-0.2 \\
-1 \cdot 1 \\
-1 \cdot 3+-1.1\end{array}$ & $\begin{array}{l}239 \\
282+-23 \\
305 \\
261+-24\end{array}$ & $\begin{array}{l}342 \\
349+- \\
337 \\
349+-\end{array}$ \\
\hline $\begin{array}{l}\mathrm{S} 2 \\
42^{\circ} 46^{-} \mathrm{N} . \\
64^{\circ} 00^{-} \mathrm{W} .\end{array}$ & $\begin{array}{r}84 \\
334 \\
247\end{array}$ & $\begin{array}{r}30 \\
50 \\
220\end{array}$ & $\begin{array}{r}210 \\
190 \\
20\end{array}$ & $\begin{array}{l}3.1 \\
2.8+-1.1 \\
0.8+-0.4\end{array}$ & $\begin{array}{l}14 \\
81+-25 \\
354+-33\end{array}$ & $\begin{array}{l}2.6 \\
2.3+-1.3 \\
3.0+-0.6\end{array}$ & $\begin{array}{l}287 \\
325+-54 \\
217+-83\end{array}$ & $\begin{array}{l}3.1 \\
3.0+-1.0 \\
3.1+-0.4\end{array}$ & $\begin{array}{l}-2.6 \\
-2.0+-1.7 \\
-0.3+-0.2\end{array}$ & $\begin{array}{l}185 \\
277+-30 \\
217+-84\end{array}$ & $\begin{array}{l}260 \\
297+-7 \\
348+-15\end{array}$ \\
\hline
\end{tabular}

Instrument type, method of analysis and source of data are listed alphabetically by stations in Tables 3. If UMINOR $<0$, ellipse rotates clockwise 
Table 6. $N_{2}$ tidal current parameters-Continued

Table 6. N2 TIDAL CURRENT PARAMETERS--Continued

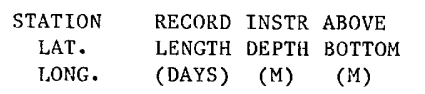

LENTTH DEPTH BOTTOM (DAY

S7

\begin{tabular}{|c|c|c|}
\hline rat & 338 & 230 \\
\hline $\begin{array}{l}42^{\circ} 42^{\prime} \mathrm{N} . \\
64^{\circ} \mathrm{O} 2^{-} \mathrm{W} .\end{array}$ & 311 & 690 \\
\hline 5 & 170 & 15 \\
\hline $43^{\circ} 34^{-} \mathrm{N}$. & 174 & 30 \\
\hline $65^{\circ} 06^{-} \mathrm{W}$. & 173 & 50 \\
\hline 1 & 162 & 15 \\
\hline $43^{\circ} 11-\mathrm{N}$. & 162 & 30 \\
\hline $65^{\circ} 43^{-W}$. & 174 & 50 \\
\hline 3 & 161 & 15 \\
\hline $42^{\circ} 50^{-} \mathrm{N}$. & 103 & 50 \\
\hline $65^{\circ} 50^{-} \mathrm{W}$. & 161 & 100 \\
\hline
\end{tabular}

NORTHEAST CHANNEL

$\begin{array}{lrrr}\mathrm{NECl} & 174 & 103 & 12 \\ 42^{\circ} 22^{-} \mathrm{N} . & 174 & 153 & 7 \\ 65^{\circ} 56^{-} \mathrm{W} . & 174 & 207 & 1 \\ & & & \\ \mathrm{NEC} 2^{*} & 58 & 106 & 13 \\ 42^{\circ} 18^{-} \mathrm{N} . & 174 & 156 & 8 \\ 65^{\circ} 58^{-} \mathrm{W} . & 58 & 217 & 17 \\ & & & \\ \mathrm{NEC} 3^{*} & 87 & 112 & 11 \\ 42^{\circ} 11^{\circ} \mathrm{N} . & 87 & 162 & 66 \\ 66^{\circ} \mathrm{O} 2^{-} \mathrm{W} . & 174 & 220 & 16\end{array}$

GULF OF MAINE

$\begin{array}{cccr} & \text { FOURIER } & \text { COEFFICIENTS } \\ \text { EAST } & \text { PHASE } & \text { NORTH } & \text { PHASE } \\ (\mathrm{CM} / \mathrm{SEC}) & (\mathrm{DEG}-\mathrm{G}) & (\mathrm{CM} / \mathrm{SEC}) & (\mathrm{DEG}-\mathrm{G})\end{array}$

1.0 $82+-29$

1.0

$154+-\quad 2$

$1.4+-1.0 \quad 122+-41$

2.7

318

2.0

213

0.8

$1.3 \quad 129$

$16.4+-0.4 \quad 124+-2 \quad 4.2+-0.7 \quad 324 t-10$

$14.6+-0.4 \quad 112+-2 \quad 3.8+-0.7 \quad 296+-10$

$9.3+-2.3 \quad 123+-14 \quad 2.8+-0.5 \quad 312+-11$

$\begin{array}{rlllll}9.8+-0.3 & 159+- & 2 & 5.2+-0.6 & 353+- & 7 \\ 10.6+-0.3 & 142+- & 2 & 7.3+-0.4 & 345+- & 3\end{array}$

$8.9+-0.5 \quad 142+-3 \quad 5.5+-0.5 \quad 335+-5$

\begin{tabular}{|c|c|}
\hline & \\
\hline $\begin{array}{r}\text { UMAJOR } \\
(\mathrm{CM} / \mathrm{SEC})\end{array}$ & $\begin{array}{r}\text { UMINOR } \\
\text { (CM/SEC) }\end{array}$ \\
\hline
\end{tabular}

PARAMETERS

PHASE ORIEN

(DEG-G) (DEG-TRUE)

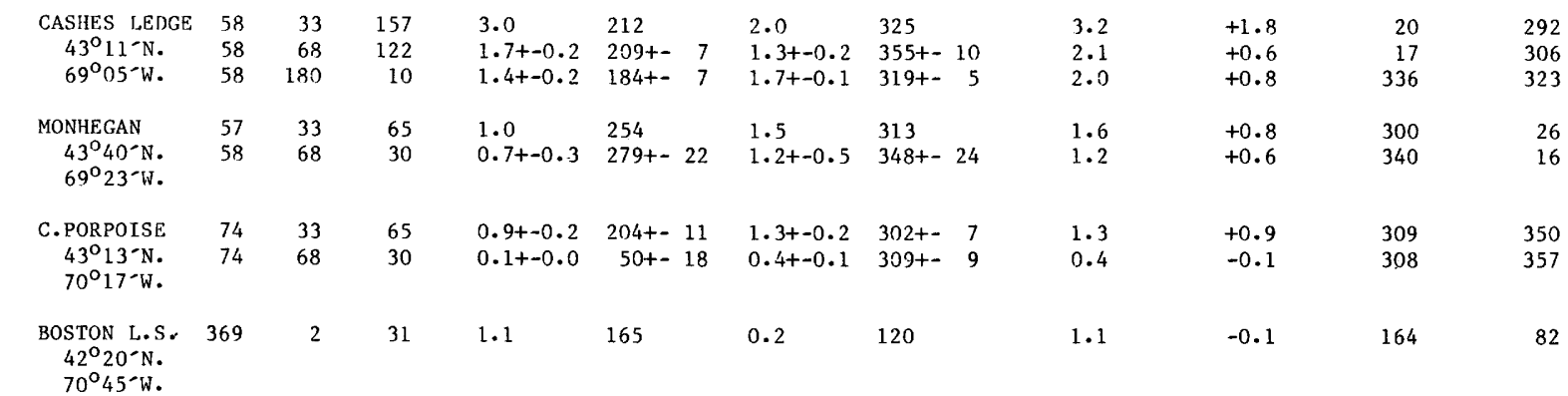

Instrument type, method of analysis and source of data are listed alphabetically by stations in Tables 3. If UMINOR $<0$, ellipse rotates clockwise

$8.5+-1.0 \quad 141+-11 \quad 6.9+-1.7 \quad 345+-16$

$9.4 t-1.9 \quad 126+-9 \quad 8.9+-2.1 \quad 346+-9$

$5.9+-2.6 \quad 120+-20 \quad 7.1+-1.7 \quad 322+-17$

$8.3+-0.9 \quad 138+-17 \quad 5.4+-2.4 \quad 353+-9$

$9.3+-1.1 \quad 126+-7 \quad 3.4 t-1.6 \quad 353+-12$

$9.1+-2.7 \quad 152+-50 \quad 10.0+-2.1 \quad 13+-45$

$11.6+-0.4 \quad 141+-11 \quad 7.3+-1.0 \quad 358+-9$

$7.9+-0.9 \quad 118+-3 \quad 10.1+1.3 \quad 343+-7$

$\begin{array}{rrrr}3.9+-1.3 & 98+-31 & 9.0+-2.2 & 320+-7\end{array}$
$10.6+-1.1$ $12 \cdot 2+-2.1$ $9.0+-1.8$

$9.4+-1.3$

$11 \cdot 5+-1.3$

$12.7+-3 \cdot 3$

$13.1+-0.3$

$11.9+-0.9$

$9.6+-1.9$
$-0.6$

267

$-0.8$

-0.8
+0.1
+1.3

$-1.4$

-1.4
-0.3
-0.4

$-1.1$

$-2.4$

$-2 \cdot 4+-2.0$

$-4.5+-1.4$

$-1.9+-2.6$

$-2.9+-2.8$

$-5.0+-1.5$

$-4.6+-0.7$

$-3.9+-1.7$

$-4 \cdot 8+-1.2$

$-2 \cdot 2+-1.5$
$331+-8308+-7$

$324+-5312+-9$

$314+-7322+-13$

$329+-6300+-7$

$326+-7$ 311+- 6

$355+-45$ 319+- 4

$331+-8300+-3$

$327+-8324+-6$

$316+-6 \quad 341+-12$ 
Table 6. $\mathrm{N}_{2}$ tidal current parameters-Continued

Table 6. N2 TIDAL CURRENT PARAMETERS--Continued

\begin{tabular}{|c|c|c|c|c|c|c|c|c|c|c|c|}
\hline STATION & RECORD & INSTR & ABOVE & & OURIER & COEFF ICIENTS & & $\mathrm{CU}$ & ENT ELLIPSE & PARAMETERS & \\
\hline $\begin{array}{l}\text { LAT. } \\
\text { LONG. }\end{array}$ & $\begin{array}{l}\text { LENGTH } \\
\text { (DAYS) }\end{array}$ & $\begin{array}{l}\text { DEPTH } \\
\text { (M) }\end{array}$ & $\begin{array}{l}\text { BOTTOM } \\
\text { (M) }\end{array}$ & $\begin{array}{c}\text { EAST } \\
(\mathrm{CM} / \mathrm{SEC})\end{array}$ & $\begin{array}{c}\text { PHASE } \\
(D E G-G)\end{array}$ & $\begin{array}{c}\text { NORTH } \\
(\mathrm{CM} / \mathrm{SEC})\end{array}$ & $\begin{array}{r}\text { PHASE } \\
\text { (DEG-G) }\end{array}$ & $\begin{array}{r}\text { UMAJOR } \\
\text { (CM/SEC) }\end{array}$ & $\begin{array}{l}\text { UMINOR } \\
(\mathrm{CM} / \mathrm{SEC})\end{array}$ & $\begin{array}{r}\text { PHASE } \\
(\mathrm{DEG}-\mathrm{G})\end{array}$ & $\begin{array}{c}\text { ORIEN } \\
\text { (DEG-TRUE) }\end{array}$ \\
\hline
\end{tabular}

BAY OF FUNDY

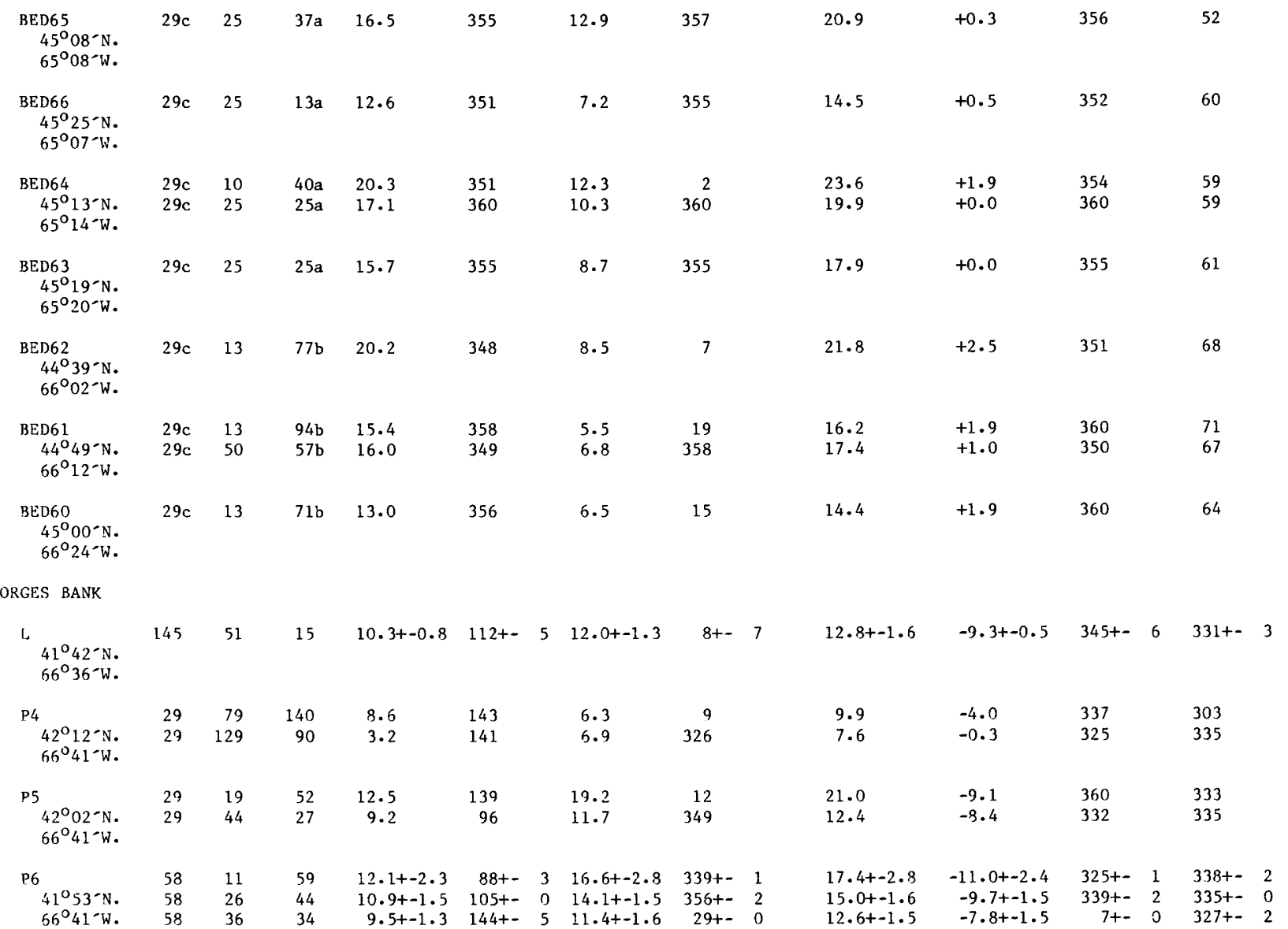

Instrument type, method of analysis and source of data are listed alphabetically by stations in Table 3.

If UMINOR < 0 , ellipse rotates clockwise

a-water depth estimated from DMA chart 14040 (old 609)

b-water depth wstimated from DMA chart 13102

c-estimated to be at least 29 -days 
Table 6. $\mathrm{N}_{2}$ tidal current parameters-Continued

Table 6. N2 TIDAL CURRENT PARAMETERS--Continued

STATION
LAT.
LONG.

RECORD INSTR ABOVE

RECORD INSTR ABOVE

(DAYS) (M) (M)

$\begin{array}{ccc}\text { EAST } & \underset{\text { POURIER }}{\text { PLASE }} & \text { COEFFICIENTS } \\ \text { (CM/SEC) } & \text { (DEG-G) } & \text { (CM/SEC) }\end{array}$

PHASE

\begin{tabular}{cccc}
\multicolumn{2}{c}{ CURRENT ELLIPSE } & PARAMETERS \\
UMANOR & UMINOR & PHASE & ORIEN \\
$(\mathrm{CM} / \mathrm{SEC})$ & (CH/SEC) & (DEG-G) & (DEG-TRUE)
\end{tabular}

GEORGES BANK--CONT.

\begin{tabular}{|c|c|c|c|c|c|c|c|c|c|c|c|c|c|c|}
\hline $\begin{array}{l}M 4^{*} \\
40^{\circ} 56^{\prime} \mathrm{N} . \\
66^{\circ} 58^{\prime} \mathrm{W} .\end{array}$ & $\begin{array}{l}58 \\
58 \\
58\end{array}$ & $\begin{array}{r}6 \\
39 \\
73\end{array}$ & $\begin{array}{r}67 \\
41 \\
8\end{array}$ & $\begin{array}{l}6.1 \\
6.5 \\
4.5\end{array}$ & $\begin{array}{r}114 \\
123 \\
81\end{array}$ & $\begin{array}{l}8.2 \\
8.3 \\
6.5\end{array}$ & $\begin{array}{r}11 \\
19 \\
350\end{array}$ & & $\begin{array}{l}8.4+-1.8 \\
8.6+-1.6 \\
6.5+-2.2\end{array}$ & $\begin{array}{l}-5.8+-2.0 \\
-6.1+-1.4 \\
-4.5+-1.8\end{array}$ & $\begin{array}{r}358+ \\
3+ \\
348+-\end{array}$ & $\begin{array}{l}16 \\
10 \\
20\end{array}$ & $\begin{array}{l}342 \\
338 \\
358\end{array}$ & \\
\hline $\begin{array}{l}\text { P1 } \\
42^{\circ} 12^{\prime} \mathrm{N} . \\
67^{\circ} 15^{\prime} \mathrm{w} .\end{array}$ & $\begin{array}{l}15 \\
15 \\
15\end{array}$ & $\begin{array}{l}30 \\
40 \\
75\end{array}$ & $\begin{array}{l}173 \\
163 \\
128\end{array}$ & $\begin{array}{l}9.3 \\
9.0 \\
6.9\end{array}$ & $\begin{array}{l}176 \\
188 \\
134\end{array}$ & $\begin{array}{l}4.8 \\
4.3 \\
6.2\end{array}$ & $\begin{array}{l}79 \\
88 \\
27\end{array}$ & & $\begin{array}{l}9.3 \\
9.0 \\
7.5\end{array}$ & $\begin{array}{l}-4.8 \\
-4.2 \\
-5.5\end{array}$ & $\begin{array}{r}358 \\
11 \\
341\end{array}$ & & $\begin{array}{l}275 \\
276 \\
305\end{array}$ & \\
\hline $\begin{array}{l}\mathrm{P2} \\
42^{\circ} 03^{\prime} \mathrm{N} . \\
67^{\circ} 15^{\prime} \mathrm{H} .\end{array}$ & $\begin{array}{l}30 \\
15\end{array}$ & $\begin{array}{l}14 \\
30\end{array}$ & $\begin{array}{l}36 \\
20\end{array}$ & $\begin{array}{l}10.8+-1.3 \\
9.1\end{array}$ & $\begin{array}{l}131+-11 \\
132\end{array}$ & $\begin{array}{l}17.4 t-0.3 \\
14.9\end{array}$ & $\begin{array}{l}357 t \\
360 t\end{array}$ & $\begin{array}{l}6 \\
8\end{array}$ & $\begin{array}{l}19.2+-0.5 \\
16.3\end{array}$ & $\begin{array}{l}-7.1+-0.1 \\
-6.2\end{array}$ & $\begin{array}{l}347+ \\
350\end{array}$ & 4 & $\begin{array}{l}333+- \\
334\end{array}$ & 6 \\
\hline $\begin{array}{l}\text { P3 } \\
41^{\circ} 3^{\prime} N . \\
67^{\circ} 15^{\prime} \mathrm{W} .\end{array}$ & $\begin{array}{l}15 \\
15 \\
15\end{array}$ & $\begin{array}{l}15 \\
30 \\
40\end{array}$ & $\begin{array}{r}30 \\
15 \\
5\end{array}$ & $\begin{array}{r}10.5 \\
10.1 \\
5.8\end{array}$ & $\begin{array}{l}106 \\
118 \\
193\end{array}$ & $\begin{array}{l}17.8 \\
16.0 \\
11.0\end{array}$ & $\begin{array}{r}7 \\
10 \\
96\end{array}$ & & $\begin{array}{l}18.1 \\
16.6 \\
11.1\end{array}$ & $\begin{array}{l}-9.9 \\
-9.3 \\
-5.8\end{array}$ & $\begin{array}{r}2 \\
1 \\
94\end{array}$ & & $\begin{array}{l}351 \\
344 \\
355\end{array}$ & \\
\hline $\begin{array}{l}\mathrm{M3}^{*} \\
\quad 41^{\circ} 0_{2} \mathrm{~N} . \\
67^{\circ} 16^{\prime} \mathrm{W} .\end{array}$ & 58 & 36 & 8 & 9.2 & 93 & 14.5 & 355 & & $14.6 t-1.0$ & $-9.0+-0.9$ & $350+$ & 4 & 352 & \\
\hline $\begin{array}{l}M 9^{*} \\
40^{\circ} 54^{\prime} \mathrm{N} \\
67^{\circ} 24^{\prime} \mathrm{W}\end{array}$ & 58 & 71 & 8 & 3.6 & 81 & 5.4 & 336 & & $5.5+-1.4$ & $-3.4+-1.0$ & $326+$ & 16 & 344 & \\
\hline $\begin{array}{l}40^{\circ} 51^{\prime} \mathrm{N} . \\
67^{\circ} 24^{\prime} \mathrm{W} .\end{array}$ & $\begin{array}{l}261 \\
957 \\
957 \\
290\end{array}$ & $\begin{array}{l}15 \\
45 \\
75 \\
84\end{array}$ & $\begin{array}{r}70 \\
40 \\
10 \\
1\end{array}$ & $\begin{array}{l}6.3+-1.5 \\
6.6+1.0 \\
4.6+-0.9 \\
3.2+-0.8\end{array}$ & $\begin{array}{r}103+18 \\
102+8 \\
87+12 \\
80+21\end{array}$ & $\begin{array}{l}7.7+-1.3 \\
7.9+-1.2 \\
6.0+-1.2 \\
4.6+-0.8\end{array}$ & $\begin{array}{l}358 t \\
354 t- \\
335 t- \\
333 t\end{array}$ & $\begin{array}{r}15 \\
9 \\
14 \\
11\end{array}$ & $\begin{array}{l}8.2+-1.4 \\
8.5+-1.2 \\
6.5+-1.2 \\
4.9+-0.8\end{array}$ & $\begin{array}{l}-5.7+-1.4 \\
-5.8+-0.9 \\
-4.0+-0.9 \\
-2.8+-0.7\end{array}$ & $\begin{array}{l}337+ \\
332+ \\
316+ \\
320+\end{array}$ & $\begin{array}{r}18 \\
9 \\
16 \\
12\end{array}$ & $\begin{array}{l}332+- \\
330+ \\
332+ \\
340+\end{array}$ & $\begin{array}{r}14 \\
10 \\
9 \\
15\end{array}$ \\
\hline $\begin{array}{l}\text { C } \\
41^{\circ} 24^{\prime} \mathrm{N} \\
67^{\circ} 34^{\prime} \mathrm{H} .\end{array}$ & 116 & 15 & 23 & $11.7+-0.8$ & $86+$ & $14.4+-1.0$ & $345+$ & 2 & $14.3+-1.1$ & $-11.1+-0.7$ & $328+$ & 3 & $339+$ & 2 \\
\hline $\begin{array}{l}\mathrm{K}^{* \hbar} \\
41^{\circ} 04^{\prime} \mathrm{N} . \\
67^{\circ} 34^{\prime} \mathrm{W} .\end{array}$ & $\begin{array}{r}58 \\
174 \\
58 \\
174 \\
87 \\
232\end{array}$ & $\begin{array}{l}10 \\
15 \\
34 \\
54 \\
58 \\
60\end{array}$ & $\begin{array}{r}54 \\
45 \\
30 \\
10 \\
4 \\
1\end{array}$ & $\begin{array}{l}9.4+-1.7 \\
8.7+-1.0 \\
9.4+-1.7 \\
6.5+-0.9 \\
6.5+-1.1 \\
4.4+-0.6\end{array}$ & $\begin{array}{l}107+5 \\
109+10 \\
107+5 \\
92+11 \\
94+9 \\
82+8\end{array}$ & $\begin{array}{r}9.3+-1.2 \\
11.0+-1.3 \\
9.3+-1.2 \\
8.4+-0.9 \\
8.1+-1.0 \\
6.4+-0.4\end{array}$ & $\begin{array}{l}5+ \\
1+ \\
5+ \\
344+ \\
347+ \\
336+\end{array}$ & $\begin{array}{r}11 \\
9 \\
11 \\
14 \\
6 \\
7\end{array}$ & $\begin{array}{r}10.2+-1.2 \\
11.6+-1.1 \\
10.2+-1.2 \\
8.8+-1.0 \\
8.6+-1.3 \\
6.6+0.5\end{array}$ & $\begin{array}{l}-8.3+-1.8 \\
-7.8+-1.2 \\
-8.3+-1.7 \\
-5.9+-1.0 \\
-5.8+-0.8 \\
-4.1+-0.5\end{array}$ & $\begin{array}{l}324+ \\
341+ \\
323+ \\
327+ \\
329+ \\
324 t\end{array}$ & $\begin{array}{r}1 \\
7 \\
1 \\
12 \\
7 \\
6\end{array}$ & $\begin{array}{l}312+- \\
332+ \\
312+ \\
335+ \\
334+- \\
342+\end{array}$ & $\begin{array}{l}9 \\
8 \\
9 \\
3 \\
4 \\
5\end{array}$ \\
\hline $\begin{array}{l}\text { D } \\
41^{\circ} 59^{\prime} \mathrm{N} . \\
67^{\circ} 47^{\prime} \mathrm{W} .\end{array}$ & 87 & 15 & 69 & $7.9+0.7$ & $109+4$ & $8.5+-1.2$ & $345+$ & 3 & $10.4 t-0.6$ & $-5.4+-0.3$ & $320+$ & 6 & $319+$ & 10 \\
\hline
\end{tabular}

Instrument type, method of analysio and source of data are 11sted alphabetically by stations in Table 3.

If UMINOR < 0 , ellipse rotatea clockwlse

H = harmonic analyals

R - response analysis

* - depths have been calculated from the mean presaure and error estimates are taken from across-1sobath

and along-1sobath directions since these are nearly the sane as the ellipse axis. These records apan 1dentical time periods.

* tripod and aubaurface woorings were at different deptha 
Table 6. $\mathrm{N}_{2}$ tidal current parameters-Continued

Table 6. N2 TIDAL CURRENT PARAMETERS--Continued

STATION RECORD INSTR ABOVE

LAT. LENGTH DEPTH BOTTOM

LONG. (DAYS) (M) (M)

GREAT SOUTH CHANNEL

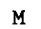

$14051-\mathrm{N} . \quad 145 \quad 10-\mathrm{H} \quad 56$

$10-\mathrm{R} \quad 56$

$\begin{array}{rrrr}68^{\circ} 49^{-W} . & 116 & 51-\mathrm{H} & 15 \\ 124 & 51-\mathrm{R} & 15\end{array}$

$40^{\circ} 49^{-} \mathrm{N}$

$$
87 \quad 58 \quad 20
$$

$69^{\circ} 00^{-W}$.

$\begin{array}{llll}\mathrm{GSC} 2 & 109 & 10 & 73 \\ 40^{\circ} 51^{-\mathrm{N}} . & 152 & 42 & 41 \\ 69^{\circ} 01^{-} \mathrm{W} . & 152 & 76\end{array}$

N

$40^{\circ} 51^{-\mathrm{N}}$

$145 \quad 68$

$69^{\circ} 01^{-W}$.

R

$40^{\circ} 30^{-} \mathrm{N}$. $69^{\circ} 07^{-W}$.

GSCl $149 \quad 27$

$40^{\circ} 52-\mathrm{N}$.

$69^{\circ} 11-\mathrm{W}$

$\begin{array}{cccr}\text { FOURIER } & \text { COEFFICIENTS } & \\ \text { EAST } & \text { PHASE } & \text { NORTH } & \text { PHASE } \\ (\mathrm{CM} / \mathrm{SEC}) & (\mathrm{DEG}-\mathrm{G}) & (\mathrm{CM} / \mathrm{SEC}) & (\mathrm{DEG}-\mathrm{G})\end{array}$

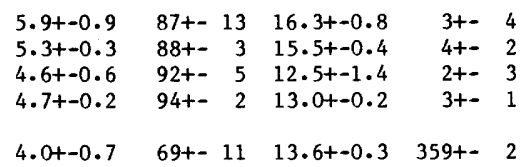

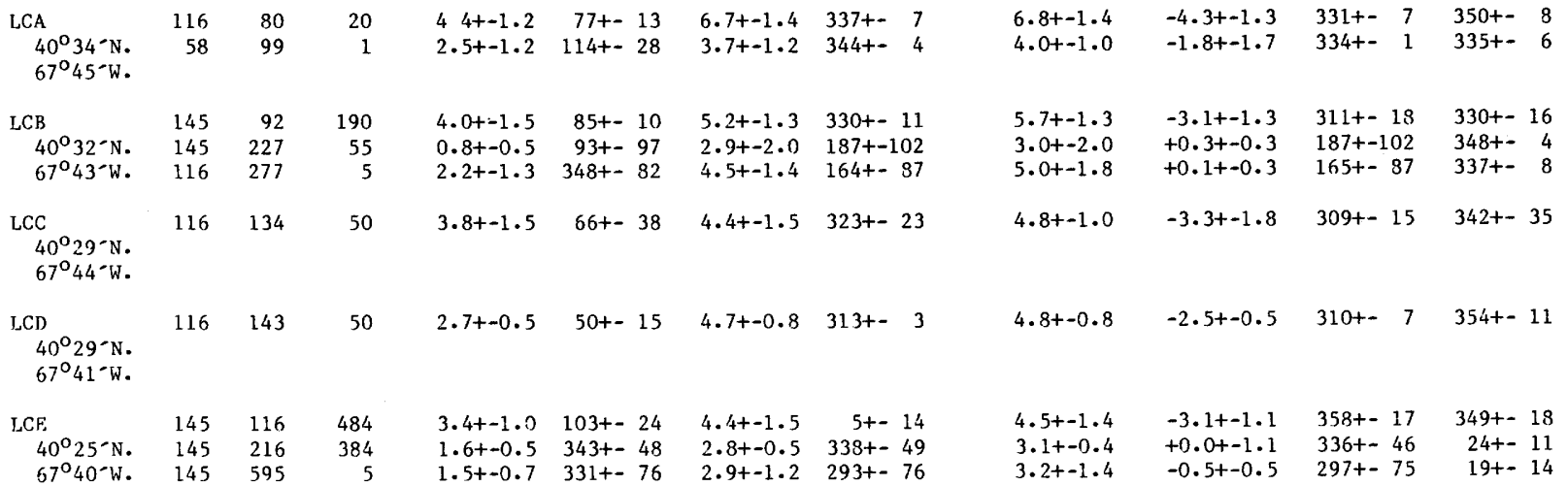

Instrument type, method of analysis and source of data are listed alphabetically by stations in Table 3 . If UMINOR < 0 , ellipse rotates clockwise.

* - Observations from stations LCB(except 277m), LCE,LCH(except 1375 and $1454 \mathrm{~m})$, LCI, LC.J(except 454m, and LCN al1 begin at 0100 on Dec. 2, 1980 and end at 0100 on Apri1 26, 1981. Observations at LCA $(80 \mathrm{~m}), \operatorname{LCB}(277 \mathrm{~m}), \mathrm{LC} J(83 \mathrm{~m}), \mathrm{LCK}(454 \mathrm{~m})$ and LCL begin at DiDo on Dec. 2, 1980 but end 29 days earlier on March 28, 1981. 
Table 6. $\mathrm{N}_{2}$ tidal current parameters-Continued

Table 6. N2 TIDAL CURRFNT PARAMETERS--Continued

STATION RECORD INSTR ABOVE

LAT. LENGTH DEPTH BOTTOM

LONG

(DAYS) (M) (M)
FOURIFR COEFFICIENTS

PHASE NORTH PHASE

$(\mathrm{CM} / \mathrm{SEC}) \quad(\mathrm{DEG}-\mathrm{G})$

\begin{tabular}{cccc}
\multicolumn{2}{c}{ CURRENT ELLIPSE } & PARAMETERS \\
UMAJOR & UMINOR & PHASE & ORIEN \\
$(\mathrm{CM} / \mathrm{SEC})$ & $(\mathrm{CM} / \mathrm{SEC})$ & $(\mathrm{DEG}-\mathrm{G})$ & $(\mathrm{DEG}-\mathrm{TRUE})$
\end{tabular}

LYDONIA CANYON--CONT.

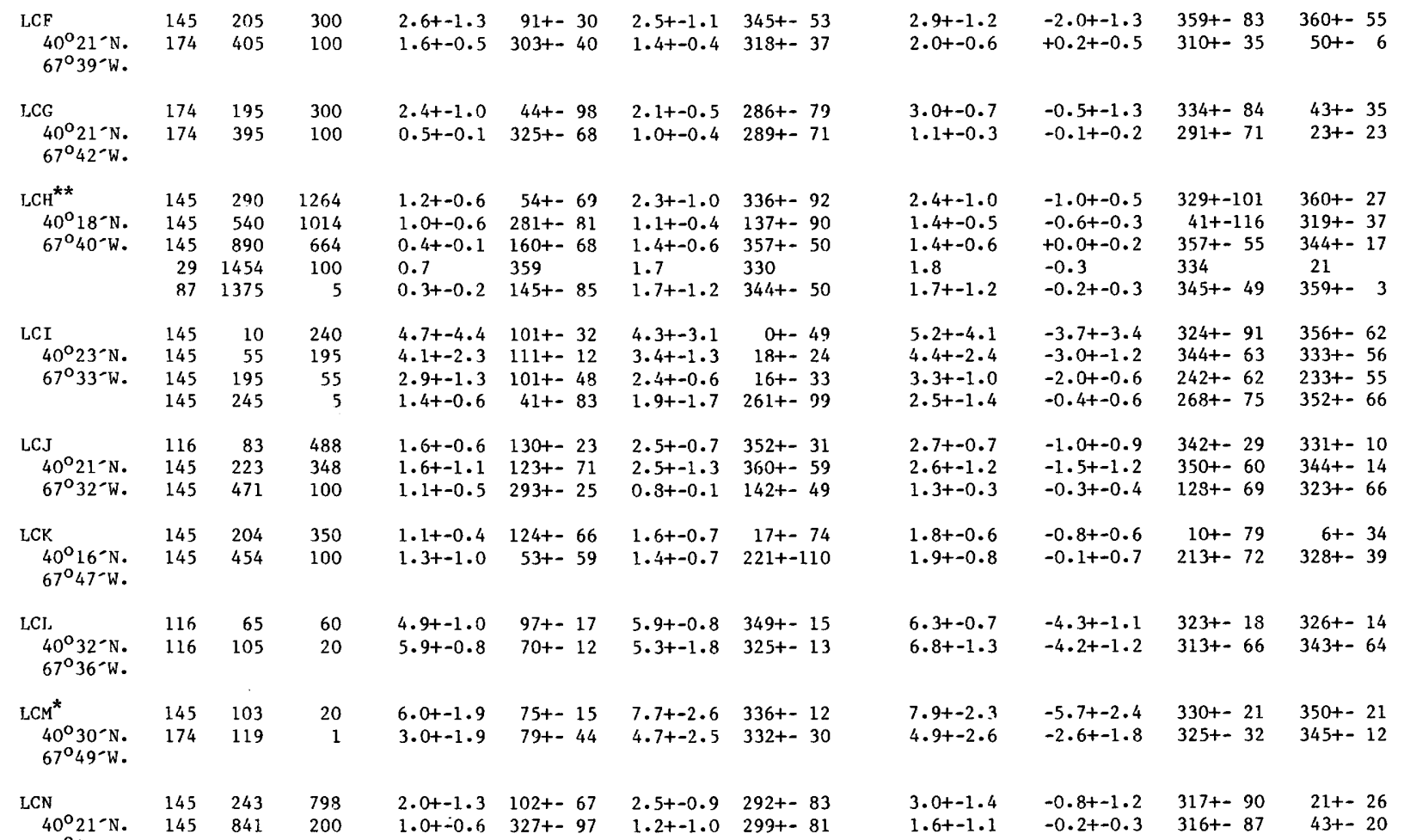

NANTUCKET SHOALS

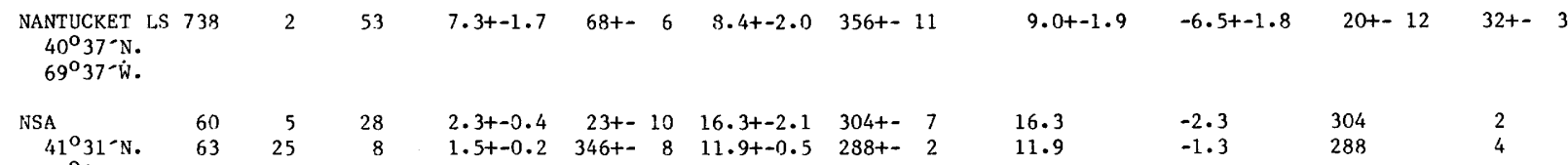

Instrument type, method of analysis and source of data are listed alphabetically by stations in Table 3 .

If UMINOR < 0 , ellipse rotates clockwise

* tripod and subsurface mooring were at different depths.

** two separate moorings at different depths 
Table 6. $\mathrm{N}_{2}$ tidal current parameters-Continued

Table 6. N2 TIDAL CURRENT PARAMETERS--Continued

\begin{tabular}{|c|c|c|c|c|c|c|c|c|c|c|c|}
\hline STATION & RECORD & INSTR & ABOVE & & DURIER & COEFFICIENTS & & $\mathrm{CU}$ & ELLIPSE & PARAMETERS & \\
\hline $\begin{array}{l}\text { LAT. } \\
\text { LONG. }\end{array}$ & $\begin{array}{l}\text { LENGTH } \\
\text { (DAYS) }\end{array}$ & $\begin{array}{l}\text { DEPTH } \\
\text { (M) }\end{array}$ & $\begin{array}{l}\text { BОтTOM } \\
\text { (M) }\end{array}$ & $\begin{array}{c}\text { EAST } \\
\text { (CM/SEC) }\end{array}$ & $\begin{array}{c}\text { PHASE } \\
(D E G-G)\end{array}$ & $\begin{array}{c}\text { NORTH } \\
(\mathrm{CM} / \mathrm{SEC})\end{array}$ & $\begin{array}{r}\text { PHASE } \\
(\text { DEG-G) }\end{array}$ & $\begin{array}{r}\text { UMAJOR } \\
\text { (CM/SEC) }\end{array}$ & $\begin{array}{l}\text { UMINOR } \\
\text { (CM/SEC) }\end{array}$ & $\begin{array}{r}\text { PHASE } \\
\text { (DEG-G) }\end{array}$ & $\begin{array}{c}\text { ORIEN } \\
\text { (DEG-TRUE) }\end{array}$ \\
\hline
\end{tabular}

NANTUCKET SHOALS--CONT.

\begin{tabular}{|c|c|c|c|c|c|c|c|c|c|c|c|c|c|}
\hline $\begin{array}{l}\text { NSB } \\
\qquad 41^{\circ} 26^{-} \mathrm{N} \\
69^{\circ} 44^{-} \mathrm{W}\end{array}$ & 42 & 10 & 12 & $6.8+-1.7$ & $355+-$ & 3 & $11 \cdot 0+-0.4$ & $319+-$ & 2 & 12.4 & $-3 \cdot 5$ & 328 & 29 \\
\hline $\begin{array}{l}\text { NSD } \\
\qquad \begin{array}{l}41^{\circ} 37^{-} \mathrm{N} . \\
69^{\circ} 44^{-} \mathrm{W} .\end{array}\end{array}$ & 42 & 16 & 17 & $5.4+-0.8$ & $292+-$ & 9 & $9.3+-0.7$ & $320+-$ & 4 & 10.5 & +2.2 & 313 & 29 \\
\hline $\begin{array}{c}\text { POLLOCK RIP } \\
41^{\circ} 37^{-} \mathrm{N} . \\
69^{\circ} 54^{-} \mathrm{W} .\end{array}$ & 369 & 2 & 12 & 3.1 & 282 & & 10.5 & 267 & & 11.0 & -0.8 & 268 & 16 \\
\hline $\begin{array}{l}\text { NSC } \\
\quad 41^{\circ} 37^{-} \mathrm{N} . \\
69^{\circ} 59^{-} \mathrm{W} .\end{array}$ & 42 & 8 & 8 & $8.4+-0.4$ & $9+-$ & 3 & $1.5+-0.2$ & $245+-$ & 6 & 8.4 & $-1 \cdot 2$ & 190 & 276 \\
\hline $\begin{array}{l}\mathrm{I} \\
40^{\circ} 43^{-} \mathrm{N} \\
70^{\circ} 01^{-} \mathrm{W}\end{array}$ & 29 & 18 & 23 & 8.4 & 37 & & 6.0 & 310 & & 8.4 & -6.0 & 34 & 86 \\
\hline $\begin{array}{l}\text { NSE } \\
\quad 40^{\circ} 59^{-} \mathrm{N} . \\
70^{\circ} 04^{-} \mathrm{W} .\end{array}$ & 41 & 10 & 12 & $14.9+-4.2$ & $333+-$ & 16 & $13.2+-3.0$ & $245+-$ & 13 & 14.9 & -13.2 & 326 & 82 \\
\hline $\begin{array}{l}\text { NSFE1 } \\
\quad 40^{\circ} 41^{\circ} \mathrm{N} . \\
70^{\circ} 08^{-} \mathrm{W} .\end{array}$ & $\begin{array}{l}172 \\
222\end{array}$ & $\begin{array}{l}10 \\
30\end{array}$ & $\begin{array}{l}36 \\
16\end{array}$ & $\begin{array}{l}6.1+-0.2 \\
5.5+-0.1\end{array}$ & $\begin{array}{l}57+- \\
35+-\end{array}$ & $\begin{array}{l}2 \\
2\end{array}$ & $\begin{array}{l}5.3+-0.2 \\
4.7+-0.2\end{array}$ & $\begin{array}{l}322+- \\
360+-\end{array}$ & $\begin{array}{l}2 \\
2\end{array}$ & $\begin{array}{l}6.2 \\
6.9\end{array}$ & $\begin{array}{l}-5 \cdot 2 \\
-2 \cdot 1\end{array}$ & $\begin{array}{l}71 \\
21\end{array}$ & $\begin{array}{r}107 \\
51\end{array}$ \\
\hline $\begin{array}{l}40^{\circ} 30^{-} \mathrm{N} \\
70^{\circ} 13^{-} \mathrm{W}\end{array}$ & $\begin{array}{l}174 \\
464 \\
377 \\
145 \\
116\end{array}$ & $\begin{array}{l}10 \\
31 \\
51 \\
57 \\
66\end{array}$ & $\begin{array}{r}57 \\
36 \\
16 \\
10 \\
1\end{array}$ & $\begin{array}{l}3.8+-0.5 \\
3.8+-0.6 \\
4.0+-0.6 \\
3.5+-1.2 \\
2.4+-0.4\end{array}$ & $\begin{array}{l}51+- \\
52+- \\
42+- \\
32+- \\
11+-\end{array}$ & $\begin{array}{r}8 \\
8 \\
8 \\
24 \\
6\end{array}$ & $\begin{array}{l}3.7+-0.7 \\
3.7+-0.5 \\
3.7+-0.7 \\
3.2+-1.7 \\
2.1+-0.4\end{array}$ & $\begin{array}{l}322+- \\
327+- \\
322+- \\
302+- \\
284+-\end{array}$ & $\begin{array}{l}24 \\
12 \\
13 \\
25 \\
10\end{array}$ & $\begin{array}{l}4.3+-0.5 \\
4.1+-0.5 \\
4.3+-0.7 \\
3.7+-1.4 \\
2.5+-0.3\end{array}$ & $\begin{array}{l}-3.2+-0.7 \\
-3.3+-0.4 \\
-3.3+-0.7 \\
-3.0+-1.5 \\
-2.0+-0.4\end{array}$ & $\begin{array}{r}44+-42 \\
40+-38 \\
27+-25 \\
40+-37 \\
351+-38\end{array}$ & $\begin{array}{l}81+-53 \\
75+-44 \\
69+-30 \\
96+-43 \\
67+-33\end{array}$ \\
\hline $\begin{array}{l}\text { NSFE3 } \\
\qquad 40^{\circ} 20^{-} \mathrm{N} . \\
70^{\circ} 16^{-\mathrm{W}} .\end{array}$ & $\begin{array}{r}46 \\
209 \\
209\end{array}$ & $\begin{array}{l}10 \\
30 \\
70\end{array}$ & $\begin{array}{l}78 \\
58 \\
18\end{array}$ & $\begin{array}{l}2.3+-0.3 \\
2 \cdot 2+-0.1 \\
3.1+-0.2\end{array}$ & $\begin{array}{l}90+- \\
60+- \\
34+-\end{array}$ & $\begin{array}{r}10 \\
3 \\
3\end{array}$ & $\begin{array}{l}2.4+-0.5 \\
2.1+-0.1 \\
2.9+-0.3\end{array}$ & $\begin{array}{r}17+- \\
336+- \\
315+-\end{array}$ & $\begin{array}{r}13 \\
3 \\
5\end{array}$ & $\begin{array}{l}2.7 \\
2.3 \\
3.3\end{array}$ & $\begin{array}{l}-2.0 \\
-2.0 \\
-2.7\end{array}$ & $\begin{array}{r}50 \\
30 \\
4\end{array}$ & $\begin{array}{l}41 \\
57 \\
55\end{array}$ \\
\hline $\begin{array}{l}\text { NSFE4 } \\
\quad 40^{\circ} 13^{\circ} \mathrm{N} . \\
70^{\circ} 18^{-} \mathrm{W} .\end{array}$ & $\begin{array}{l}170 \\
224 \\
170 \\
224\end{array}$ & $\begin{array}{l}10 \\
30 \\
60 \\
90\end{array}$ & $\begin{array}{l}95 \\
75 \\
45 \\
15\end{array}$ & $\begin{array}{l}2.8+-0.4 \\
2.1+-0.2 \\
1.8+-0.2 \\
1.3+-0.1\end{array}$ & $\begin{array}{l}11+- \\
57+- \\
71+- \\
56+-\end{array}$ & $\begin{array}{l}9 \\
5 \\
5 \\
6\end{array}$ & $\begin{array}{l}3.1+-0.5 \\
2.7+-0.3 \\
1.9+-0.2 \\
1.1+-0.1\end{array}$ & $\begin{array}{l}280+- \\
332+- \\
353+- \\
337+-\end{array}$ & $\begin{array}{l}9 \\
6 \\
5 \\
7\end{array}$ & $\begin{array}{l}3.1 \\
2.7 \\
2.0 \\
1.3\end{array}$ & $\begin{array}{l}-2.7 \\
-2.1 \\
-1.6 \\
-1.0\end{array}$ & $\begin{array}{r}274 \\
339 \\
22 \\
37\end{array}$ & $\begin{array}{r}354 \\
10 \\
34 \\
66\end{array}$ \\
\hline $\begin{array}{l}\text { NSFE } 5^{*} \\
40^{\circ} 02^{-N} \text {. } \\
70^{\circ} 22^{\prime} \mathrm{W} .\end{array}$ & $\begin{array}{l}171 \\
171 \\
171 \\
171 \\
171\end{array}$ & $\begin{array}{r}10 \\
30 \\
90 \\
120 \\
185\end{array}$ & $\begin{array}{r}188 \\
168 \\
108 \\
78 \\
13\end{array}$ & $\begin{array}{l}1.3+-0.6 \\
1.2 \\
0.5+-0.2 \\
0.9+-0.2 \\
1.9+-0.6\end{array}$ & $\begin{array}{c}126+- \\
131 \\
4+- \\
13+- \\
54+-\end{array}$ & $\begin{array}{l}26 \\
19 \\
12 \\
17\end{array}$ & $\begin{array}{l}1.6+-0.7 \\
1.7 \\
0.6+-0.2 \\
1.2+-0.3 \\
2.3+-0.7\end{array}$ & $\begin{array}{l}51+- \\
37 \\
261+- \\
265+- \\
324+-\end{array}$ & $\begin{array}{l}26 \\
\\
24 \\
15 \\
17\end{array}$ & $\begin{array}{l}1.7 \\
1.8 \\
0.6 \\
1.2 \\
2.3\end{array}$ & $\begin{array}{l}-1.2 \\
-1.2 \\
-0.4 \\
-0.8 \\
-1.9\end{array}$ & $\begin{array}{r}67 \\
33 \\
249 \\
249 \\
323\end{array}$ & $\begin{array}{r}22 \\
354 \\
343 \\
336 \\
360\end{array}$ \\
\hline
\end{tabular}

Instrument type, method of analysis and source of dat are listed alphabetically by stations in Table 3 . If UMINOR < 0, ellipse rotates clockwise

* Orientation is questionahle 
Table 6. $\mathrm{N}_{2}$ tidal current parameters-Continued

Table 6. N2 TIDAL CURRENT PARAMETERS--Continued

STATION RECORD INSTR ABOVE

LAT. LENGTH DEPTH BOTTOM

LONG. (DAYS) (M) (M)

$\begin{array}{cccr} & \text { FOURIER } & \text { COEFFICIENTS } & \\ \text { EAST } & \text { PHASE } & \text { NORTH } & \text { PHASE } \\ (\mathrm{CM} / \mathrm{SEC}) & (\mathrm{DEG}-\mathrm{G}) & (\mathrm{CM} / \mathrm{SEC}) & (\mathrm{DEG}-\mathrm{G})\end{array}$

NANTUCKET SHOALS --CONT.

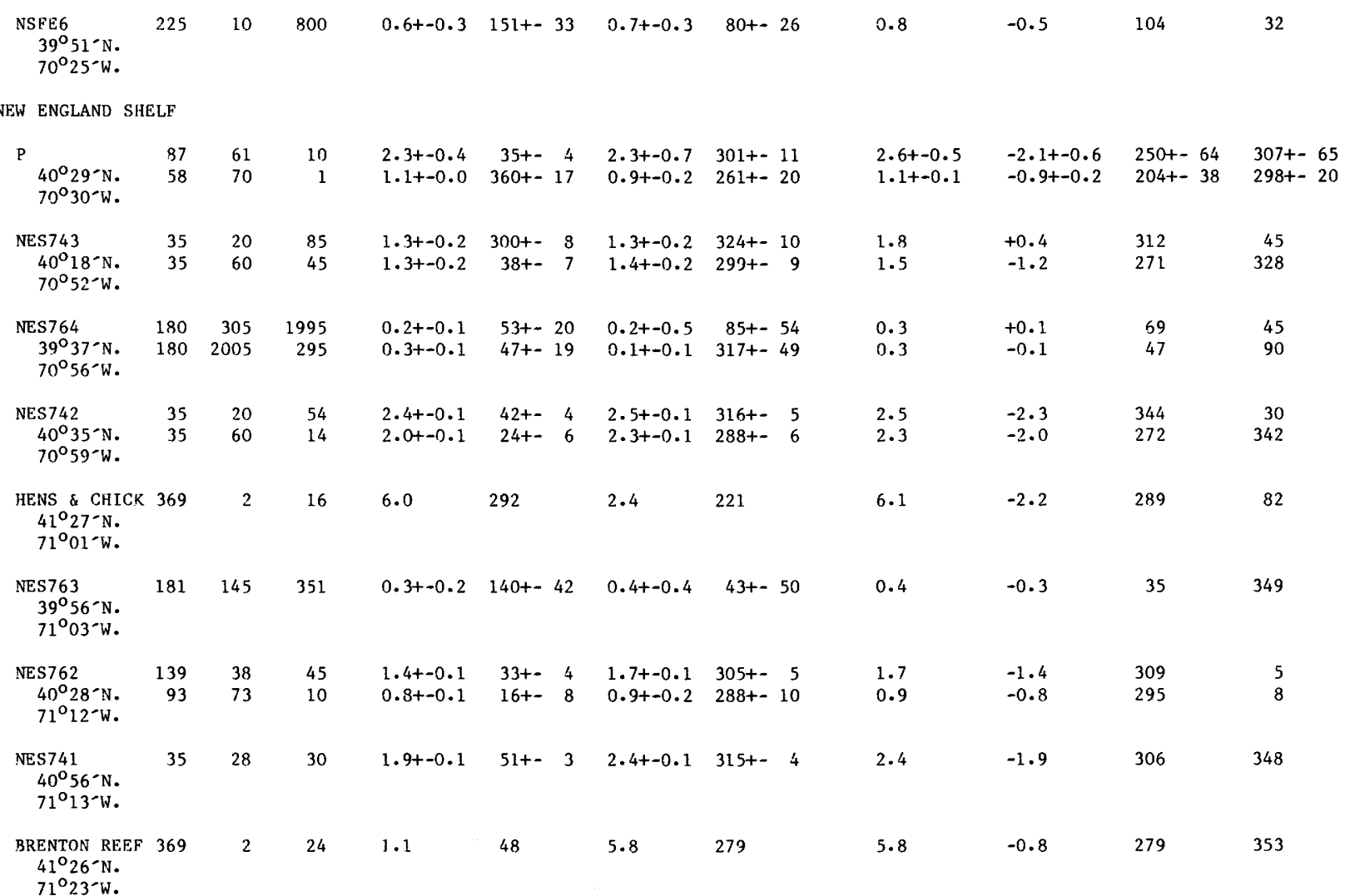

\begin{tabular}{cccc}
\multicolumn{2}{c}{ CURRENT ELLIPSE } & PARAMETERS \\
UMAJOR & UMINOR & PHASE & ORIEN \\
$(\mathrm{CM} / \mathrm{SEC})$ & $(\mathrm{CM} / \mathrm{SEC})$ & (DEG-G) & (DEG-TRUE)
\end{tabular}

Instrument type, method of analysis and source of data are listed alphabetically by stations in Table 3 .

Instrument type, method of analysis and sou
If UMINOR $<0$, ellipse rotates clockwise. 
Table 6. $\mathrm{N}_{2}$ tidal current parameters-Continued

Table 6. N2 TIDAL CURRENT PARAMETERS--Continued

$\begin{array}{cl}\text { STATION } & \text { RECORD INSTR ABOVE } \\ \text { LAT. } & \text { LENGTH DEPTH BOTTOM }\end{array}$

LONG.

(DAYS) (H) (M)
COEFFICIENTS

NORTH
$(\mathrm{CH} / \mathrm{SEC}) \quad(\mathrm{DEG}-\mathrm{G})$

\begin{tabular}{|c|c|c|c|}
\hline & ELLIPSE & PARAMETERS & \\
\hline $\begin{array}{l}\text { UMAJOR } \\
\mathrm{CM} / \mathrm{SEC} \text { ) }\end{array}$ & $\begin{array}{c}\text { UMINOR } \\
\text { (CM/SEC) }\end{array}$ & $\begin{array}{r}\text { PHASE } \\
(\text { DEG-G) }\end{array}$ & $\begin{array}{c}\text { ORIEN } \\
\text { (DEG-TRUE }\end{array}$ \\
\hline
\end{tabular}

MIDDLE ATLANTIC BIGHT

\begin{tabular}{|c|c|c|c|c|c|c|c|c|c|c|c|c|c|}
\hline $\begin{array}{l}\text { NES763H } \\
39^{\circ} 43^{-N} . \\
71^{\circ} 47 \mathrm{~W} .\end{array}$ & 183 & 302 & 202 & $0.8+-0.3$ & $43+-$ & 19 & $0.7+-0.3$ & 299+- & 24 & 0.8 & -0.6 & 247 & 301 \\
\hline $\begin{array}{l}\text { NES762W } \\
39^{\circ} 55^{-N} . \\
71^{\circ} 58-\mathrm{W} .\end{array}$ & 172 & 38 & 45 & $1.9+-0.1$ & $34+-$ & 4 & $1.8+-0.1$ & $281+-$ & 5 & 2.2 & $-1 \cdot 4$ & 244 & 311 \\
\hline $\begin{array}{l}\text { LT5 } \\
40^{\circ} 12-\mathrm{N} . \\
72^{\circ} 00^{-} \mathrm{W}\end{array}$ & $\begin{array}{r}87 \\
70 \\
116 \\
70 \\
87 \\
70 \\
87 \\
70\end{array}$ & $\begin{array}{l}21-H \\
21-R \\
41-R \\
41-R \\
61-R \\
61-R \\
66-B \\
66-R\end{array}$ & $\begin{array}{r}46 \\
46 \\
26 \\
26 \\
6 \\
6 \\
1 \\
1\end{array}$ & $\begin{array}{l}2.4+-0.4 \\
1.7+-0.2 \\
2.6+-0.9 \\
2.2+-0.2 \\
1.3+-0.1 \\
1.2+-0.1 \\
1.1+-0.3 \\
0.9+-0.1\end{array}$ & $\begin{array}{r}40+- \\
42+- \\
30+- \\
27+- \\
17+- \\
15+- \\
3+- \\
22+-\end{array}$ & $\begin{array}{r}22 \\
6 \\
65 \\
6 \\
12 \\
5 \\
39 \\
6\end{array}$ & $\begin{array}{l}2.4+-0.3 \\
2.1+-0.3 \\
2.3+-1.4 \\
1.6+-0.2 \\
1.4+-0.4 \\
1.1+-0.2 \\
1.2+-0.7 \\
0.6+-0.1\end{array}$ & $\begin{array}{l}279+- \\
284+- \\
278+- \\
284+- \\
257+- \\
256+- \\
244+- \\
264+-\end{array}$ & $\begin{array}{r}16 \\
8 \\
44 \\
8 \\
12 \\
8 \\
35 \\
10\end{array}$ & $\begin{array}{l}3.0+-0.4 \\
2.3 \\
3.1+-1.2 \\
2.3 \\
1.7+-0.4 \\
1.4 \\
1.5+-0.5 \\
1.0\end{array}$ & $\begin{array}{l}-1.7+-0.3 \\
-1.3 \\
-1.8+-1.0 \\
-1.5 \\
-0.9+-0.2 \\
-0.8 \\
-0.8+-0.4 \\
-0.5\end{array}$ & $\begin{array}{l}248+-21 \\
264 \\
263+-16 \\
219 \\
230+-1 \\
221 \\
215+-30 \\
215\end{array}$ & $\begin{array}{l}314+- \\
327 \\
331+- \\
287 \\
319+- \\
310 \\
315+- \\
294\end{array}$ \\
\hline $\begin{array}{l}\text { LT4 } \\
40^{\circ} 34-\mathrm{N} . \\
72^{\circ} 19-\mathrm{H} .\end{array}$ & $\begin{array}{r}29 \\
203 \\
174 \\
87\end{array}$ & $\begin{array}{r}3 \\
24 \\
44 \\
51\end{array}$ & $\begin{array}{r}49 \\
28 \\
8 \\
1\end{array}$ & $\begin{array}{l}2.6+-0.3 \\
4.3+-1.6 \\
2.7+-0.5 \\
1.1+-0.9\end{array}$ & $\begin{array}{l}47+- \\
44+- \\
34+- \\
39+-\end{array}$ & $\begin{array}{r}7 \\
22 \\
22 \\
35\end{array}$ & $\begin{array}{l}0.9+-0.5 \\
2.4+-1.4 \\
1.6+-0.6 \\
0.6+-0.6\end{array}$ & $\begin{array}{l}326+- \\
294+- \\
298+- \\
292+-\end{array}$ & $\begin{array}{l}28 \\
34 \\
23 \\
43\end{array}$ & $\begin{array}{l}2.6+-0.3 \\
4.5+-1.6 \\
2.8+-0.5 \\
1.1+-1.0\end{array}$ & $\begin{array}{l}-0.9+-0.4 \\
-2.1+-1.4 \\
-1.6+-0.6 \\
-0.5+-0.5\end{array}$ & $\begin{array}{l}225+-3 \\
230+-29 \\
215+-27 \\
226+-31\end{array}$ & $\begin{array}{l}265+- \\
281+- \\
273+- \\
282+-\end{array}$ \\
\hline $\begin{array}{l}\text { CMICE } \\
40^{\circ} 47-\mathrm{N} . \\
72^{\circ} 29^{-} \mathrm{W} .\end{array}$ & $\begin{array}{l}25 \\
25 \\
25 \\
25\end{array}$ & $\begin{array}{r}4 \\
8 \\
16 \\
25\end{array}$ & $\begin{array}{r}25 \\
21 \\
13 \\
4\end{array}$ & $\begin{array}{l}2.2+-0.3 \\
2.4+-0.2 \\
2.2+-0.2 \\
1.5+-0.1\end{array}$ & $\begin{array}{l}46+- \\
37+- \\
41+- \\
14+-\end{array}$ & $\begin{array}{l}8 \\
6 \\
5 \\
6\end{array}$ & $\begin{array}{l}0.2+-0.1 \\
0.4+-0.2 \\
0.6+-0.3 \\
0.9+-0.4\end{array}$ & $\begin{array}{l}336+- \\
259+- \\
260+- \\
204+-\end{array}$ & $\begin{array}{l}31 \\
29 \\
14 \\
27\end{array}$ & $\begin{array}{l}2.2 \\
2.4 \\
2.3 \\
1.7\end{array}$ & $\begin{array}{l}-0.2 \\
-0.3 \\
-0.4 \\
-0.1\end{array}$ & $\begin{array}{l}226 \\
218 \\
223 \\
197\end{array}$ & $\begin{array}{l}268 \\
277 \\
282 \\
301\end{array}$ \\
\hline $\begin{array}{l}\mathrm{ME} \\
39^{\circ} 57^{\circ} \mathrm{N} \\
72^{\circ} 36^{-} \mathrm{W}\end{array}$ & 29 & 59 & 1 & 1.1 & 30 & & 1.8 & 229 & & 2.1 & -0.3 & 223 & 328 \\
\hline $\begin{array}{l}\mathrm{NJ4} \\
38^{\circ} 55^{-\mathrm{N}} \\
72^{\circ} 58^{-\mathrm{W}}\end{array}$ & $\begin{array}{l}72 \\
72 \\
72\end{array}$ & $\begin{array}{r}3 \\
43 \\
91\end{array}$ & $\begin{array}{r}89 \\
49 \\
1\end{array}$ & $\begin{array}{l}2.8+-0.7 \\
3.3+-0.4 \\
0.3+-0.1\end{array}$ & $\begin{array}{r}26+- \\
56+- \\
114+-\end{array}$ & $\begin{array}{r}14 \\
7 \\
24\end{array}$ & $\begin{array}{l}2.2+-0.6 \\
2.8+-0.5 \\
0.3+-0.2\end{array}$ & $\begin{array}{r}270+- \\
287+- \\
33+-\end{array}$ & $\begin{array}{l}16 \\
11 \\
33\end{array}$ & $\begin{array}{l}3.1 \\
3.9 \\
0.3\end{array}$ & $\begin{array}{l}-1.8 \\
-1.8 \\
-0.3\end{array}$ & $\begin{array}{l}225 \\
256 \\
254\end{array}$ & $\begin{array}{l}301 \\
308 \\
225\end{array}$ \\
\hline $\begin{array}{l}\mathrm{MA} \\
39^{\circ} 27^{-N} \\
73^{\circ} \mathrm{OO}-\mathrm{W}\end{array}$ & 58 & 58 & 1 & $1.7+-1.1$ & $21+-$ & 24 & $1.6+-0.1$ & $267+-$ & 41 & $2.1+-0.6$ & $-1.1+-0.7$ & $228+-$ & $311+-$ \\
\hline $\begin{array}{l}\text { LT3 } \\
39^{\circ} 16^{\circ} \mathrm{N} \\
73^{\circ} 02^{-} \mathrm{W}\end{array}$ & $\begin{array}{r}104 \\
104 \\
70\end{array}$ & $\begin{array}{r}9 \\
19 \\
58\end{array}$ & $\begin{array}{l}61 \\
51 \\
12\end{array}$ & $\begin{array}{l}3.5+-0.3 \\
3.6+-0.4 \\
2.5+-0.3\end{array}$ & $\begin{array}{l}51+- \\
51+- \\
79+-\end{array}$ & $\begin{array}{l}5 \\
6 \\
6\end{array}$ & $\begin{array}{l}3.2+-0.3 \\
3.3+-0.3 \\
2.3+-0.3\end{array}$ & $\begin{array}{l}286+- \\
292+- \\
305+-\end{array}$ & $\begin{array}{l}6 \\
6 \\
7\end{array}$ & $\begin{array}{l}4.2 \\
4.2 \\
3.1\end{array}$ & $\begin{array}{l}-2.2 \\
-2.5 \\
-1.3\end{array}$ & $\begin{array}{l}255 \\
257 \\
280\end{array}$ & $\begin{array}{l}311 \\
310 \\
312\end{array}$ \\
\hline $\begin{array}{l}\text { MESA7 } \\
39^{\circ} 55^{\circ} \mathrm{N} . \\
73^{\circ} 06^{-} \mathrm{W} .\end{array}$ & $\begin{array}{r}107 \\
103 \\
63\end{array}$ & $\begin{array}{l}18 \\
38 \\
66\end{array}$ & $\begin{array}{r}50-a \\
30-a \\
2-a\end{array}$ & $\begin{array}{l}3.7+-0.4 \\
3.6+-0.4 \\
0.7+-0.1\end{array}$ & $\begin{array}{l}49+- \\
56+- \\
87+-\end{array}$ & $\begin{array}{r}6 \\
5 \\
12\end{array}$ & $\begin{array}{l}2.6+-0.4 \\
1.7+-0.2 \\
1.1+-0.2\end{array}$ & $\begin{array}{l}324+- \\
293+- \\
276+-\end{array}$ & $\begin{array}{l}8 \\
8 \\
8\end{array}$ & $\begin{array}{l}3.7 \\
3.7 \\
1.3\end{array}$ & $\begin{array}{l}-2.6 \\
-1.4 \\
-0.1\end{array}$ & $\begin{array}{l}224 \\
242 \\
273\end{array}$ & $\begin{array}{l}263 \\
287 \\
328\end{array}$ \\
\hline
\end{tabular}

Instrument type, nethod of analysis and source of data are 1isted alphabetically by stations in Table 3.

Instrument type, wethod of analysis and source of data are 11sted alphabetically by stations in Table 3.

If UMINOR < 0, ell1pse rotates clockwise

$H$ - harmonic analysis $R$ - response analysis

a - water depth taken frow DMA chart 13200 
Table 6. $\mathrm{N}_{2}$ tidal current parameters-Continued

Table 6. N2 TIDAL CURRENT PARAMETERS--Continued

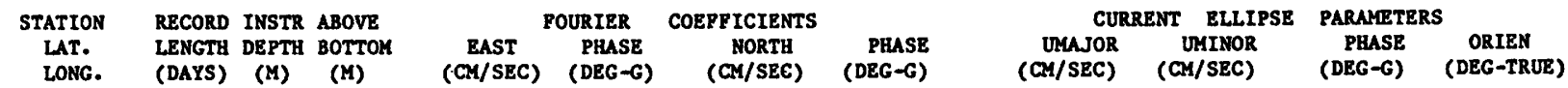

MIDDLE ATLANTIC BIGHT--CONT.

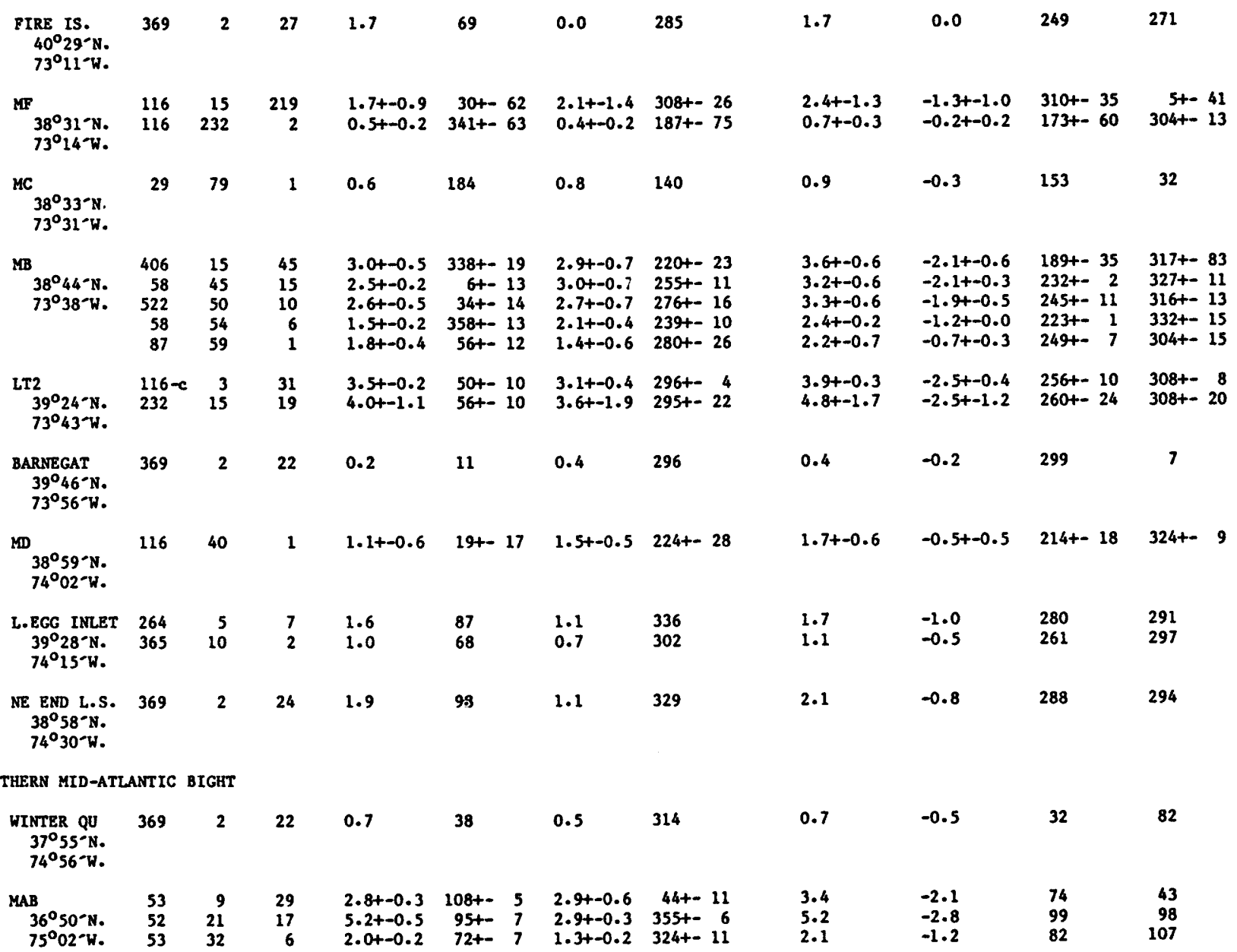

Instrunent type, method of analysis and source of data are listed alphabetically by stations in Table 3. If UMINOR < 0 , ellipse rotates clockwise

c - average of 4 records o $31,31,32$ and 29 above bottom 
Table 6. $\mathrm{N}_{2}$ tidal current parameters-Continued

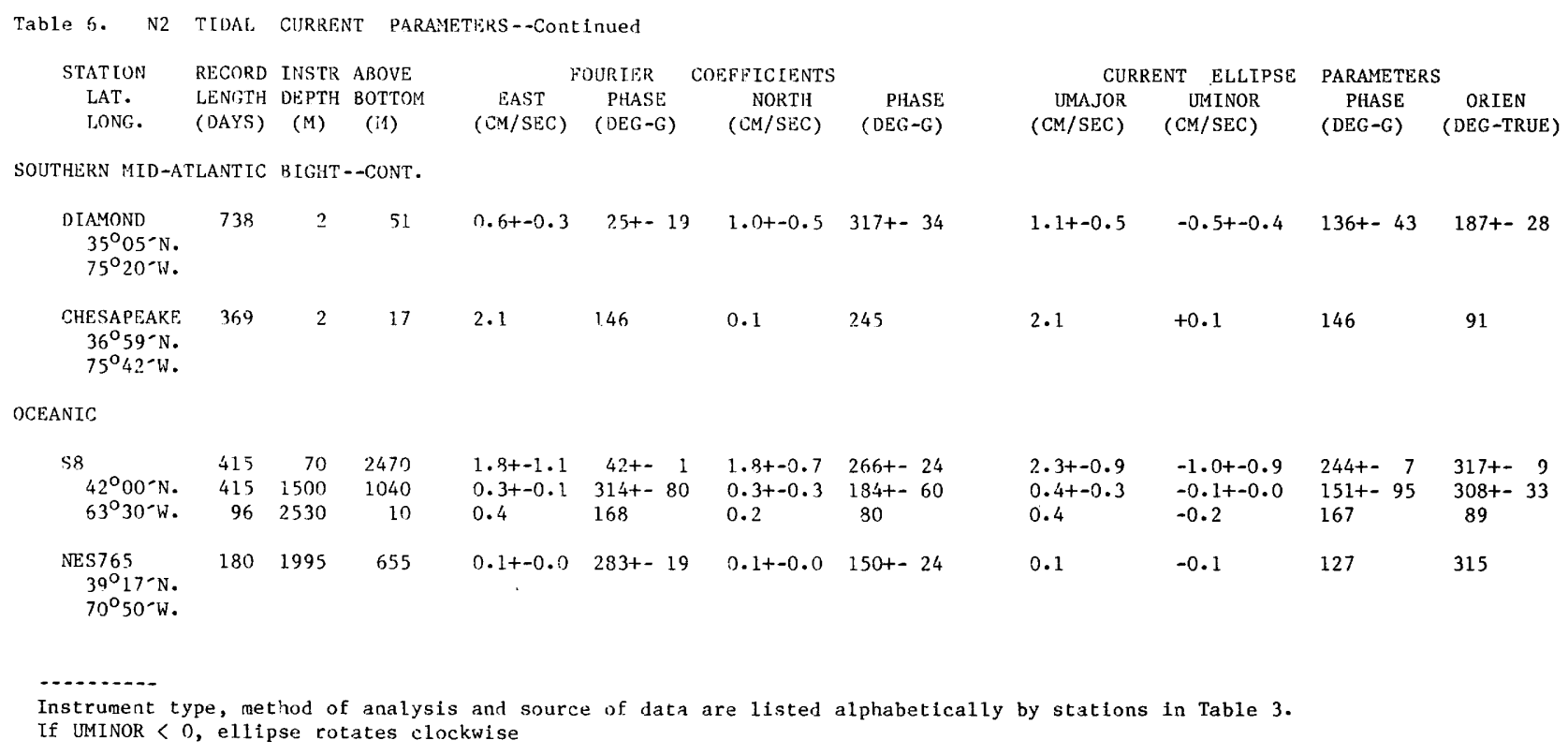


Table 7. $\mathrm{S}_{2}$ tidal current parameters (see table 5 for explanation).

Table 7. S2 TIDAL CURRENT PARAMETERS

STATION RECORD INSTR ABOVE

LAT. LENGTH DEPTH BOTTOM
LONG. (DAYS) (M) (M)

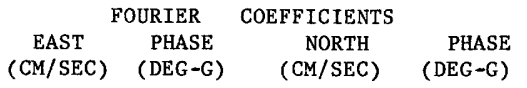

$(\mathrm{CM} / \mathrm{SEC}) \quad(\mathrm{DEG}-\mathrm{G})$

SCOTIAN SHELF

\begin{tabular}{|c|c|c|c|c|c|c|c|c|c|c|c|}
\hline $\begin{array}{l}\operatorname{ss} 10 \\
\quad 43^{\circ} 34-\mathrm{N} . \\
59^{\circ} 04^{-W} .\end{array}$ & $\begin{array}{l}32 \\
22\end{array}$ & $\begin{array}{l}200 \\
500\end{array}$ & $\begin{array}{l}1400 \\
1100\end{array}$ & $\begin{array}{l}0.8 \\
0.5\end{array}$ & $\begin{array}{l}179 \\
246\end{array}$ & $\begin{array}{l}0.8 \\
0.1\end{array}$ & $\begin{array}{r}42 \\
274\end{array}$ & $\begin{array}{l}1.1 \\
0.5\end{array}$ & $\begin{array}{l}-0.4 \\
+0.0\end{array}$ & $\begin{array}{r}21 \\
247\end{array}$ & $\begin{array}{r}315 \\
80\end{array}$ \\
\hline $\begin{array}{l}\text { SS2A } \\
\quad 42^{\circ} 52^{-} \mathrm{N} \\
62^{\circ} 00^{-} \mathrm{W}\end{array}$ & 35 & 555 & 245 & 0.9 & 271 & 1.5 & 193 & 1.5 & -0.9 & 199 & 11 \\
\hline $\begin{array}{l}\text { SS8 } \\
\quad 42^{\circ} 37^{-} \mathrm{N} . \\
62^{\circ} 05^{-} \mathrm{W} .\end{array}$ & 36 & 1500 & 50 & 0.6 & 123 & 0.6 & 335 & 0.8 & -0.2 & 319 & 315 \\
\hline $\begin{array}{l}\text { SSIA } \\
\quad 42^{\circ} 52^{-} \mathrm{N} . \\
62^{\circ} 14^{-} \mathrm{W}\end{array}$ & $\begin{array}{l}35 \\
35\end{array}$ & $\begin{array}{l}290 \\
490\end{array}$ & $\begin{array}{r}260 \\
60\end{array}$ & $\begin{array}{l}1.5 \\
0.3\end{array}$ & $\begin{array}{r}240 \\
46\end{array}$ & $\begin{array}{l}1.9 \\
1.3\end{array}$ & $\begin{array}{l}218 \\
271\end{array}$ & $\begin{array}{l}2.4 \\
1.4\end{array}$ & $\begin{array}{l}-0.4 \\
-0.3\end{array}$ & $\begin{array}{l}226 \\
267\end{array}$ & $\begin{array}{r}38 \\
344\end{array}$ \\
\hline $\begin{array}{l}\mathrm{SS} 3 \\
\quad 43^{\circ} 22^{-} \mathrm{N} . \\
62^{\circ} 40^{-} \mathrm{W} .\end{array}$ & $\begin{array}{r}238 \\
149 \\
47 \\
15 \\
136\end{array}$ & $\begin{array}{l}20 \\
50 \\
81 \\
91 \\
95\end{array}$ & $\begin{array}{r}79 \\
49 \\
18 \\
8 \\
4\end{array}$ & $\begin{array}{l}3.6+-1.2 \\
2.4+-0.7 \\
1.5 \\
0.6 \\
1.9+-0.7\end{array}$ & $\begin{array}{l}96+-36 \\
49+-14 \\
75 \\
318 \\
41+-19\end{array}$ & $\begin{array}{l}4.9+-2.1 \\
3.6+-1.1 \\
3.3 \\
0.5 \\
3.0+-0.4\end{array}$ & $\begin{array}{l}339+-24 \\
304+-\quad 8 \\
317 \\
217 \\
289+-13\end{array}$ & $\begin{array}{l}5.4+-2.0 \\
3.8+-1.1 \\
3.4 \\
0.6 \\
3.1+-0.4\end{array}$ & $\begin{array}{l}-2.7+-1.4 \\
-2.2+-0.8 \\
-1.3 \\
-0.5 \\
-1.7+-0.6\end{array}$ & $\begin{array}{l}323+-43 \\
298+-18 \\
312 \\
156 \\
278+-18\end{array}$ & $\begin{array}{l}329+-24 \\
348+-17 \\
346 \\
293 \\
340+-10\end{array}$ \\
\hline $\begin{array}{l}\mathrm{SS} 7 \\
\quad 43^{\circ} 02^{\circ} \mathrm{N} . \\
62^{\circ} 54^{-} \mathrm{W} .\end{array}$ & $\begin{array}{l}27 \\
27\end{array}$ & $\begin{array}{r}50 \\
118\end{array}$ & $\begin{array}{r}75 \\
7\end{array}$ & $\begin{array}{l}1.7 \\
0.8\end{array}$ & $\begin{array}{r}90 \\
126\end{array}$ & $\begin{array}{l}2.5 \\
1.5\end{array}$ & $\begin{array}{l}324 \\
341\end{array}$ & $\begin{array}{l}2.8 \\
1.6\end{array}$ & $\begin{array}{l}-1.2 \\
-0.4\end{array}$ & $\begin{array}{l}310 \\
334\end{array}$ & $\begin{array}{l}332 \\
335\end{array}$ \\
\hline $\begin{array}{l}\text { SS2 } \\
\quad 43^{\circ} 45^{-} \mathrm{N} \\
62^{\circ} 59^{-} \mathrm{W}\end{array}$ & $\begin{array}{l}189 \\
283 \\
166 \\
259\end{array}$ & $\begin{array}{r}20 \\
50 \\
95 \\
250\end{array}$ & $\begin{array}{r}258 \\
228 \\
183 \\
28\end{array}$ & $\begin{array}{l}1.0+-1.5 \\
0.7+-0.2 \\
0.6+-0.1 \\
0.6+-0.4\end{array}$ & $\begin{array}{r}90+-46 \\
278+-101 \\
23+-58 \\
91+-69\end{array}$ & $\begin{array}{l}0.8+-0.5 \\
1.2+-0.9 \\
1.2+-0.6 \\
1.2+-0.6\end{array}$ & $\begin{array}{l}360+-34 \\
317+-37 \\
292+-39 \\
323+-25\end{array}$ & $\begin{array}{l}1.3+-1.4 \\
1.4+-0.7 \\
1.3+-0.6 \\
1.3+-0.6\end{array}$ & $\begin{array}{l}-0.5+-0.5 \\
-0.2+-0.4 \\
-0.5+-0.1 \\
-0.4+-0.5\end{array}$ & $\begin{array}{l}350+-63 \\
319+-47 \\
302+-21 \\
319+-29\end{array}$ & $\begin{array}{r}4+-50 \\
3+-45 \\
11+-29 \\
340+-\quad 9\end{array}$ \\
\hline $\begin{array}{l}\text { SS6 } \\
\quad 43^{\circ} 15^{-} \mathrm{N} . \\
63^{\circ} 22^{-} \mathrm{W} .\end{array}$ & $\begin{array}{l}26 \\
27\end{array}$ & $\begin{array}{r}50 \\
130\end{array}$ & $\begin{array}{r}85 \\
5\end{array}$ & $\begin{array}{l}1.9 \\
2.2\end{array}$ & $\begin{array}{r}71 \\
153\end{array}$ & $\begin{array}{l}4.1 \\
3.7\end{array}$ & $\begin{array}{r}336 \\
15\end{array}$ & $\begin{array}{l}4.1 \\
4.1\end{array}$ & $\begin{array}{l}-1.9 \\
-1.3\end{array}$ & $\begin{array}{r}335 \\
6\end{array}$ & $\begin{array}{l}357 \\
333\end{array}$ \\
\hline $\begin{array}{l}\mathrm{SS} 1 \\
44^{\circ} 26^{-} \mathrm{N} . \\
63^{\circ} 29^{-} \mathrm{W} .\end{array}$ & $\begin{array}{l}211 \\
187\end{array}$ & $\begin{array}{l}14 \\
95\end{array}$ & $\begin{array}{r}87 \\
6\end{array}$ & $\begin{array}{l}0.8+-0.6 \\
0.5+-0.2\end{array}$ & $\begin{array}{l}108+-54 \\
279+-75\end{array}$ & $\begin{array}{l}1.3+-0.5 \\
0.5+-0.1\end{array}$ & $\begin{array}{r}354+-55 \\
76+-61\end{array}$ & $\begin{array}{l}1.5+-0.4 \\
0.6+-0.2\end{array}$ & $\begin{array}{l}-0.5+-0.4 \\
-0.0+-0.3\end{array}$ & $\begin{array}{r}335+-45 \\
86+-66\end{array}$ & $\begin{array}{l}330+-27 \\
314 t-18\end{array}$ \\
\hline $\begin{array}{l}\mathrm{S} 5 \\
42^{\circ} 30^{-} \mathrm{N} . \\
63^{\circ} 30^{-} \mathrm{W} .\end{array}$ & $\begin{array}{l}262 \\
262 \\
370\end{array}$ & $\begin{array}{r}50 \\
150 \\
1534\end{array}$ & $\begin{array}{r}1470 \\
1370 \\
10\end{array}$ & $\begin{array}{l}1.3+-0.8 \\
0.1+-0.1 \\
0.4\end{array}$ & $\begin{array}{l}346+-7 \\
223+=23 \\
116\end{array}$ & $\begin{array}{l}1.4+-0.4 \\
0.4+-0.1 \\
0.7\end{array}$ & $\begin{array}{l}236+-25 \\
208+-27 \\
318\end{array}$ & $\begin{array}{l}1.6+-0.8 \\
0.4+-0.2 \\
0.8\end{array}$ & $\begin{array}{l}-0.9+-0.3 \\
-0.0+-0.0 \\
-0.1\end{array}$ & $\begin{array}{l}217+-43 \\
210+-27 \\
313\end{array}$ & $\begin{array}{rr}332+- & 28 \\
20+- & 3 \\
331 & \end{array}$ \\
\hline
\end{tabular}

Instrument type, method of analysis and source of data are listed alphabetically by stations in Table 3.

Instrument type, method of analysis and
If UMINOR $<0$, ellipse rotates clockwise

\begin{tabular}{cccc}
\multicolumn{2}{c}{ CURRENT } & ELLIPSE & PARAMETERS \\
UMAJOR & UMINOR & PHASE & ORIEN \\
$(\mathrm{CM} / \mathrm{SEC})$ & $(\mathrm{CM} / \mathrm{SEC})$ & $($ DEG $-\mathrm{G})$ & $($ DEG-TRUE)
\end{tabular}

(DEG-G) 
Table 7. $\mathrm{S}_{2}$ tidal current parameters-Continued

Table 7. S2 TIDAL CURRENT PARAMETERS--Continued

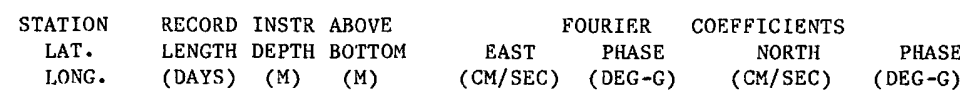

SCOTIAN SHELF--CONT.

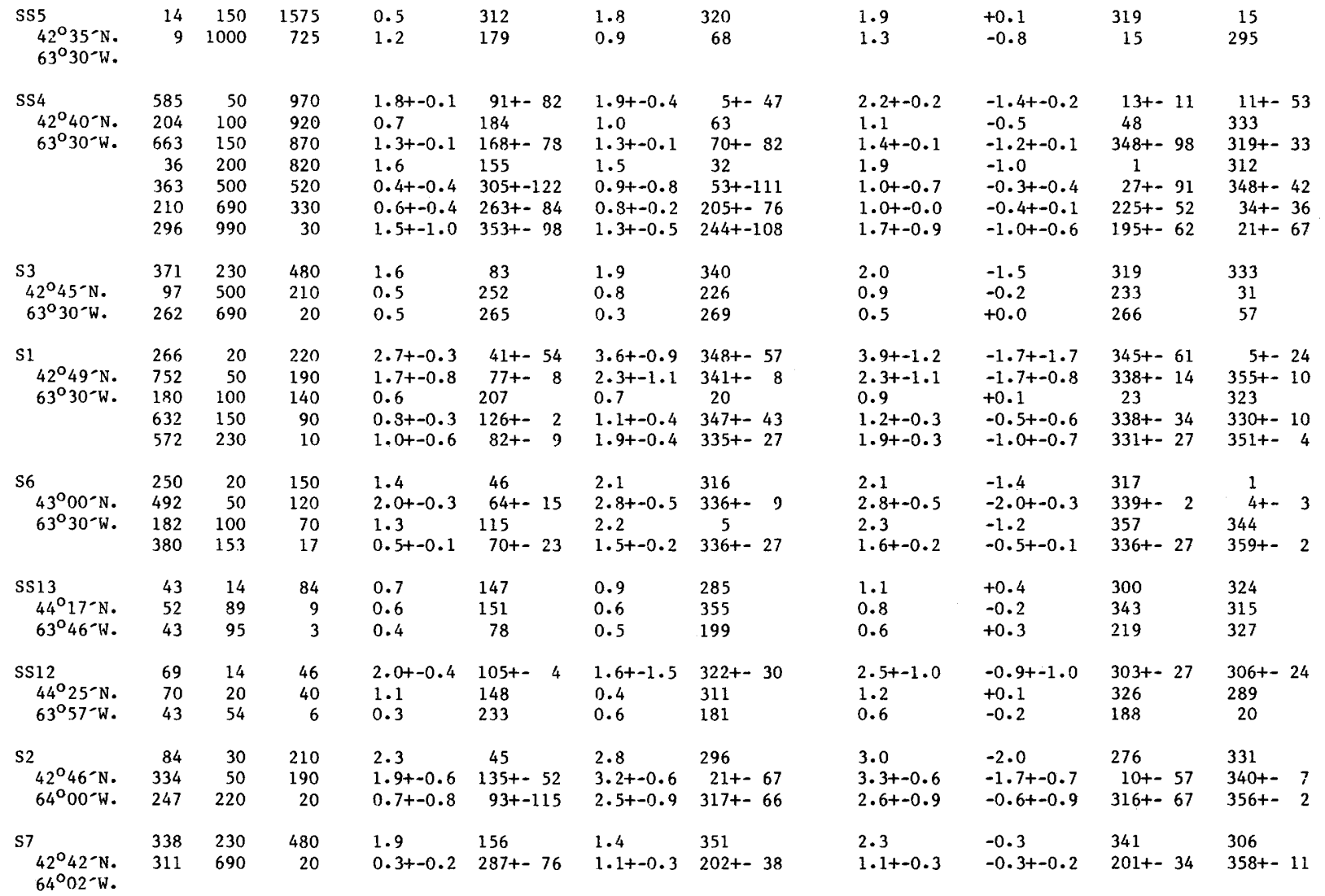

Instrument type, method of analysis and source of data are listed alphabetically by stations in Table 3.

If UMINOR $<0$, ellipse rotates clockwise 
Table 7. $\mathrm{S}_{2}$ tidal current parameters-Continued

Table 7. S2 TIDAI CURRENT PARAMETERS--Continued

\begin{tabular}{|c|c|c|c|}
\hline STATION & RECORD & INSTR & ABOVE \\
\hline $\begin{array}{l}\text { LAT. } \\
\text { LONG. }\end{array}$ & $\begin{array}{l}\text { LENGTH } \\
\text { (DAYS) }\end{array}$ & $\begin{array}{c}\text { DEPTH } \\
\text { (M) }\end{array}$ & $\begin{array}{l}\text { BOTTOM } \\
\text { (M) }\end{array}$ \\
\hline
\end{tabular}

$\begin{array}{cccr} & \text { FOURIER } & \text { COEFFICIENTS } & \\ \text { EAST } & \text { PHASE } & \text { NORTH } & \text { PHASE } \\ (\mathrm{CM} / \mathrm{SEC}) & (\mathrm{DEG}-\mathrm{G}) & (\mathrm{CM} / \mathrm{SEC}) & (\mathrm{DEG}-\mathrm{G})\end{array}$

\begin{tabular}{cccc}
\multicolumn{2}{c}{ CURRENT } & ELLIPSE & PARAMETERS \\
UMAJOR & UMINOR & PHASE & ORIEN \\
$(\mathrm{CM} / \mathrm{SEC})$ & $(\mathrm{CM} / \mathrm{SEC})$ & $(\mathrm{DEG}-\mathrm{G})$ & $(\mathrm{DEG}-\mathrm{TRUE})$
\end{tabular}

SCOTIAN SHELF--CONT.

$\begin{array}{lrrr}\mathrm{C} 5 & 170 & 15 & 45 \\ 43^{\circ} 34^{-} \mathrm{N} . & 174 & 30 & 30 \\ 65^{\circ} 06^{-} \mathrm{W} . & 173 & 50 & 10 \\ \mathrm{Cl} & & & \\ 43^{\circ} 11^{-} \mathrm{N} . & 162 & 15 & 45 \\ 65^{\circ} 43^{-} \mathrm{W} . & 174 & 50 & 30 \\ & & & 10 \\ \mathrm{C} 342^{\circ} 50^{-} \mathrm{N} . & 161 & 15 & 95 \\ 65^{\circ} 50^{-} \mathrm{W} . & 161 & 100 & 10\end{array}$

\begin{tabular}{|c|c|c|c|c|}
\hline $0.5+-0.4$ & $273+-$ & 41 & $2.6+-1.4$ & $210+-$ \\
\hline 1.8 & 113 & & 1.9 & 167 \\
\hline 1.0 & 127 & & 1.1 & 289 \\
\hline $2.3+-0.3$ & $254+-$ & 2 & $3.9+-0.7$ & $132+-$ \\
\hline $9.5+-0.3$ & $230+-$ & 2 & $2.5 t-0.4$ & $60+-$ \\
\hline $5 \cdot 8+-1.5$ & $245+-$ & 14 & $1.7+-0.3$ & $28+-$ \\
\hline $6.8+-0.2$ & $265+-$ & 2 & $3.5+-0.4$ & $79+-$ \\
\hline $8.2+$ & $245+-$ & 2 & $4 \cdot 2+-c$ & $91+-$ \\
\hline $5.6+-0.3$ & $253+-$ & 3 & $3.0+-0.3$ & $111+-$ \\
\hline
\end{tabular}

NORTHEAST CHANNEL

$\begin{array}{lrrr}\mathrm{NECl} & 174 & 103 & 120 \\ 42^{\circ} 22^{-\mathrm{N}} . & 174 & 153 & 70 \\ 65^{\circ} 56^{-\mathrm{W}} . & 174 & 207 & 16 \\ & & & \\ \mathrm{NEC} 2^{*} & 58 & 106 & 134 \\ 42^{\circ} 18^{-} \mathrm{N} . & 174 & 156 & 84 \\ 65^{\circ} 58^{-} \mathrm{W} . & 58 & 217 & 17 \\ & & & \\ \mathrm{NEC} 3^{*} & 87 & 112 & 116 \\ 42^{\circ} 11^{-} \mathrm{N} . & 87 & 162 & 66 \\ 66^{\circ} 02^{-} \mathrm{W} . & 174 & 220 & 16\end{array}$

\begin{tabular}{|c|c|}
\hline $\begin{array}{l}7.4+-0.8 \\
7.3+-1.4 \\
3.9+-1.2\end{array}$ & $\begin{array}{lr}205+- & 8 \\
199+- & 5 \\
191+- & 18\end{array}$ \\
\hline $\begin{array}{l}5.5+-0.1 \\
7.0+-1.1 \\
5.5+-2.8\end{array}$ & $\begin{array}{l}201+-5 \\
197+-10 \\
163+-62\end{array}$ \\
\hline $\begin{array}{l}8.2 t-1.6 \\
7.5+-2.4 \\
3.5+-0.5\end{array}$ & $\begin{array}{l}210+- \\
199+- \\
196+-3\end{array}$ \\
\hline
\end{tabular}

$\begin{array}{ll}6.2+-0.7 & 58+-7 \\ 7.3+-1.0 & 56+-9 \\ 5.7+-1.2 & 31+-17 \\ & \\ 6.7+-1.0 & 46+-6 \\ 6.5+-1.6 & 54+-6 \\ 7.4+-3.5 & 23+-43 \\ 7.1+-1.0 & 59+-15 \\ 8.8+-2.6 & 60+-5 \\ 6.1+-1.3 & 26+-6\end{array}$

2.6

2.3

\section{$-0.4$}

12.5

9.8

6.0

$$
-3.2
$$

-3.2
-0.4

$+1.0$

7.7

9.1

6.1

$+0.3$

$-1.6$

$-1.7$

\begin{tabular}{|c|c|c|c|}
\hline $\begin{array}{l}9.2+-0.9 \\
9.7+-1.3 \\
6.8+-0.8\end{array}$ & $\begin{array}{l}-2 \cdot 7+-1.0 \\
-3.4+-1.3 \\
-1.2+-1.8\end{array}$ & $\begin{array}{ll}38+- & 3 \\
37+- & 5 \\
24+- & 3\end{array}$ & $\begin{array}{l}309+- \\
315+- \\
325+-\end{array}$ \\
\hline $\begin{array}{l}8.5+-0.8 \\
9.0+-1.2 \\
8.6+-3.7\end{array}$ & $\begin{array}{l}-1.9+-0.0 \\
-3.1+-1.7 \\
-3.4+-2.8\end{array}$ & $\begin{array}{ll}36+- & 4 \\
35+- & 2 \\
10+- & 49\end{array}$ & $\begin{array}{l}321+- \\
312+- \\
327+-\end{array}$ \\
\hline $\begin{array}{r}10.6+-0.5 \\
10.8+-2.9 \\
6.8+-1.3\end{array}$ & $\begin{array}{l}-2.6+-1.0 \\
-4.1+-2.2 \\
-0.6+-1.7\end{array}$ & $\begin{array}{l}41+- \\
43+- \\
25+-\end{array}$ & $\begin{array}{l}310+- \\
322+- \\
332+-\end{array}$ \\
\hline
\end{tabular}

GULF OF MAINE

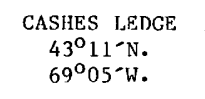

$\begin{array}{rrr}58 & 33 & 157 \\ 58 & 68 & 122 \\ 58 & 180 & 10\end{array}$

2.9

$2.9 \quad 32$

$0.6+-0.1 \quad 71+-7 \quad 0.4+-0.1 \quad 214+-10$

$0.8+-0.1 \quad 291+-7 \quad 2.0+-0.2 \quad 103+-5$

3.1
0.7

$-0.6+-1.7$

$\begin{array}{rr}211 & 5 \\ 142 & 43 \\ 117 & 137 \\ 77 & 280 \\ 51 & 285 \\ 63 & 284 \\ & \\ 84 & 297 \\ 70 & 295 \\ 80 & 295\end{array}$

$+2.0$

$\begin{array}{llll}.7 & +0.2 & 241 & 301 \\ 2 & +0.1 & 104 & 338\end{array}$

229

$229 \quad 245$

MONHEGA:

$43^{\circ} 40^{\circ} \mathrm{N}$.

$\begin{array}{llll}0.4 & 253 & 0.9 & 126\end{array}$

$69^{\circ} 23^{-W}$.

$0.6+-0.2 \quad 86+-22 \quad 1.3+-0.5 \quad 180+-24$

$\begin{array}{llll}0.9 & -0.3 & 120 & 343\end{array}$

$\begin{array}{cccc}\text { C. PORPOISE } & 74 & 33 \\ 43^{\circ} 13^{-} \mathrm{N} . & 74 & 68 \\ 70^{\circ} 17^{-} \mathrm{W} . & & \end{array}$

$0.2+-0.0 \quad 91+-11 \quad 0.8+-0.1 \quad 120+-$

$0.3+-0.1 \quad 317+-18 \quad 1.0+-0.2 \quad 73+-9$

0.8

$+0.6$

120

343
358

BOSTON L.S. $369 \quad 2 \quad 31$ $42^{\circ} 20^{-} \mathrm{N}$.

$0.8 \quad 216$

0.1

0.8

$+0.1$

$+0.3$

119

13 $70^{\circ} 45^{-\mathrm{W}}$.

-

Instrument type, method of analysis and source of data are listed alphabetically by stations in Table 3.

If UMINOR < 0 , ellipse rotates cluckwise.

* - Data frorn two separate moorings at different times. 
Table 7. $\mathrm{S}_{2}$ tidal current parameters-Continued

Table 7. S2 TIDAL CURRENT PARAMETERS--Continued

\begin{tabular}{|c|c|c|c|c|c|c|}
\hline STATION & RECORD & INSTR & ABOVE & & DURIER & COEFF ICIENTS \\
\hline LAT. & LENGTH & DEPTH & BOTTOM & EAST & PHASE & NORTH \\
\hline
\end{tabular}

\begin{tabular}{cccc}
\multicolumn{2}{c}{ CURRENT ELLIPSE } & PARAMETERS \\
UMAJOR & UMINOR & PHASE & ORIEN \\
$(\mathrm{CM} / \mathrm{SEC})$ & $(\mathrm{CM} / \mathrm{SEC})$ & (DEG-G) & (DEG-TRUE)
\end{tabular}

BAY OF FUNDY

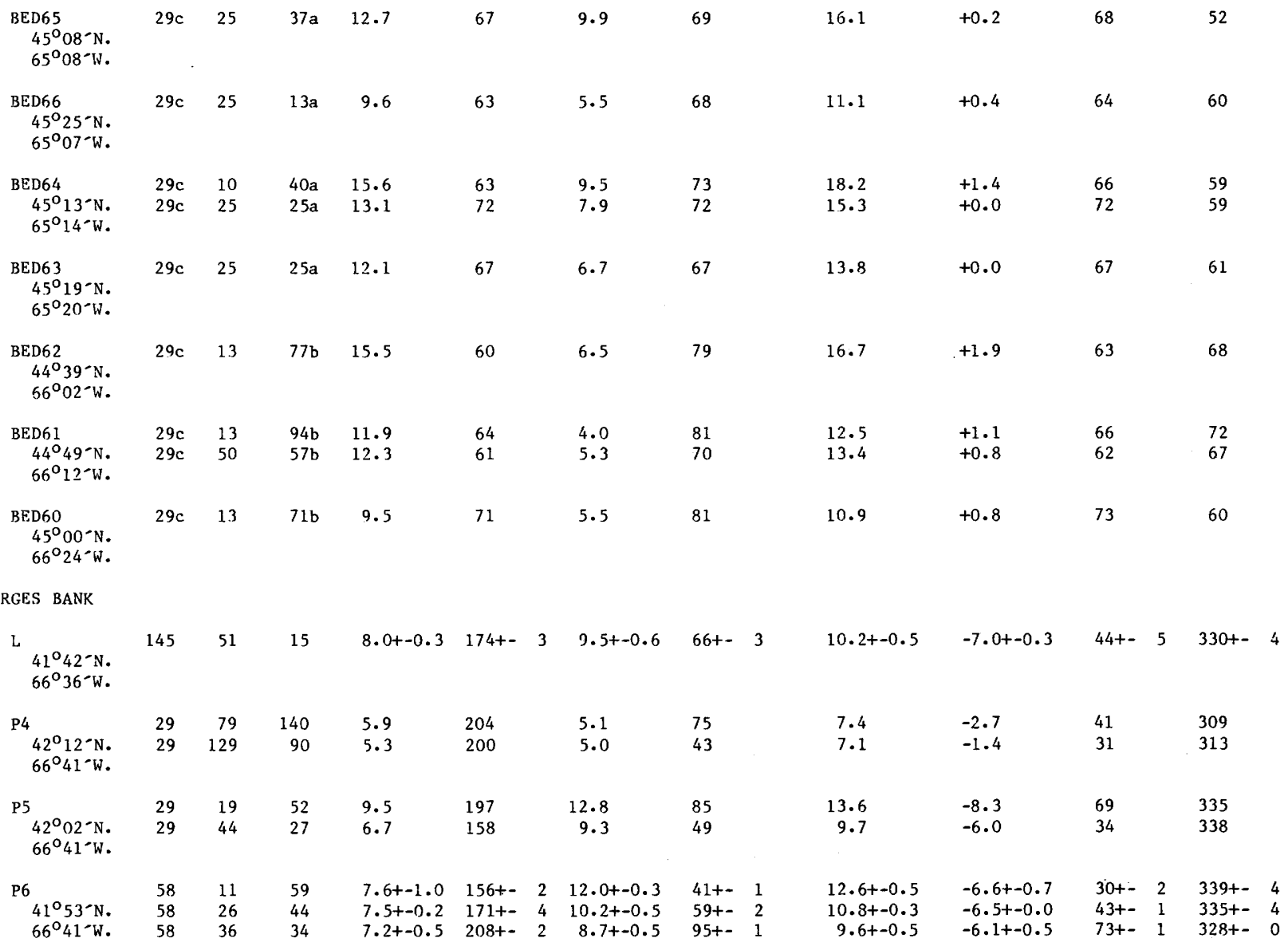

Instrument type, method of analysis and source of data are listed alphabetically by stations in Table 3 .

Instrument type, method of analysis and sour
If UMINOR $<0$, ellipse rotates clockwise.

a - water depth estimated from DMA chart 14040 (old 609)

b - water depth estimated from DMA chart 13102 .

c - estimated to be at least 29 days. 
Table 7. $\mathrm{S}_{2}$ tidal current parameters-Continued

Table 7. S2 TIDAL CURRERT PARAKETERS--Continued

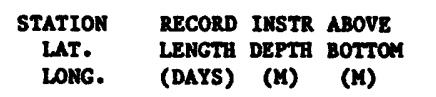

LONG. (DAYS) (M) (M)

GEORGES BANK--CONT. $\begin{array}{lll}44^{*} & 58 & 10 \\ 40^{\circ} 56^{\prime} \mathrm{N} . & 58 & 36 \\ 66^{\circ} 58^{\prime} \mathrm{W} . & 58 & 69\end{array}$ P1
$42^{\circ} 12 \mathrm{~N}$.
$67^{\circ} 15^{\prime} \mathrm{W}$.

P2 $42^{\circ} 03^{\circ} \mathrm{X}$
$67^{\circ} 15^{\circ}$ $67^{\circ} 15^{\prime}$ น.

13* $41^{\circ} 20^{\prime} \mathrm{N}$ $67^{\circ} 16$ 'W.

M9* $40^{\circ} 54$ ' $67^{\circ} 24^{\prime} \mathrm{H}$.

1 $\begin{array}{rrr}40^{\circ} 51^{\prime} \text { 'N. } & 261 & 15 \\ 67^{\circ} 24^{\prime} \text { 'W. } & 957 & 45 \\ & 290 & 75 \\ & 116 & 15 \\ & & \\ 41^{\circ} 24^{\prime} \text { 'N. } & & \\ 67^{\circ} 34^{\prime} \text { '. } & & \\ & & \\ * & 58 & 10 \\ 41^{\circ} 04^{\prime} \text { 'N. } & 174 & 15 \\ 67^{\circ} 34^{\prime} \text { 'W. } & 58 & 34 \\ & 174 & 54 \\ & 87 & 58 \\ & 232 & 60\end{array}$

D $41^{\circ} 59^{\prime} \mathrm{N}$. $87 \quad 15 \quad 69$

$\underset{\text { (CAST SBC) }}{\text { EAST }}$
FOURIER

PHASE COEFPICIERTS (DEG-G) (CM/SEC) (DEG-G)

\begin{tabular}{|c|c|c|c|}
\hline CUS & NT ELLIPSE & PARAMETERS & \\
\hline $\begin{array}{l}\text { URAJOR } \\
\text { CH/SEC) }\end{array}$ & $\begin{array}{l}\text { UNINOR } \\
\text { (CM/SEC) }\end{array}$ & $\begin{array}{l}\text { PHASE } \\
\text { (DEG-G) }\end{array}$ & $\begin{array}{c}\text { ORIEN } \\
\text { (DEG-TRU }\end{array}$ \\
\hline
\end{tabular}

$6.1+-1.3 \quad-4.9+-1.7 \quad 62+15 \quad 338$

5.6
7.0

-2.9
-2.4

$+0.6$

$54 \quad 276$

$\begin{array}{ll}3.0 & 127 \\ 2.4 & 115\end{array}$

$5.1 \quad 213$

$9.0+0.9$ 188t

$314.6+-1.7 \quad 11+2$

$16.6+-1.1$

$-4.2+-0.3$

$-3.2$

11.2

\section{$-5.7$}

$-5.1$

12.8

$-13.4$

$9.1+1.0$

$-6.1+-0.9$

$20 \quad 347$

307

5

$6.5 \quad 164$

8.8

59

4.9

73

$5.2+-1$

$-2.8+-1$

$59+17$

335

$3.4+0.9 \quad 163+34 \quad 4.2+-0.9 \quad 50+19$

$4.3+-0.5 \quad 166+10 \quad 5.3+-0.6 \quad 56+8$

$3.2+-0.7 \quad 152+15 \quad 4.4 t-0.6 \quad 37 t 13$

$\begin{array}{rr}4.4+-0.6 & 37+13 \\ 3.6+-0.2 & 27+8\end{array}$

$4.7+0.8$

$-2.6+-0.9$

$-3.7+0.6$

$5.7+-0.5$

$4.7+0.6$

$-2.7+0.7$

$-2.2+0.3$

$33+23 \quad 331+22$

$3.8+-0.2$

$-6.7+-0.2$

$35+10$ 330t 11

22+ 11 335t

$18+-634413$

$9.7+0.3$

40t 5 339t

$7.2+0.6$

$7.3+1.0$

$7.2+0.6$

$-3.6+-1.6$

$-4.7+0.7$

$-3.6+-1.6$

$-4.2+-0.8$

$-3.9+0.3$

$-3.1+-0.3$

$4.8+-0.4$

$-4.2+-1.3$

$6.2+1.3 \quad 187+8 \quad 5.8+-0.9 \quad 65+17$

$-4.2+1$ $67^{\circ} 47^{\prime} \mathrm{H}$.

Instrument type, wethod of analysis and source of data are listed alphabetically by stations in Table 3.

If $\mathrm{UMINOR}<0$, ell1pse rotates clockwlse

B - harwonic anilgeis

R - responce analyals

an the mean pressure and error estinates are taken fron acrose-1sobath and along-1sobath directione

Ince these are nearly the same as the ellipse axis. These records span identical tiee periods.

n* - tripod and eubsurface nooringe were at different depths 
Table 7. $S_{2}$ tidal current parameters-Continued

Table 7. S2 TIDAL CURRENT PARAMETERS--Continued

\begin{tabular}{|c|c|c|c|c|c|c|c|c|c|c|c|}
\hline STATION & RECORD & INSTR & ABOVE & & OURIER & COEFFICIENTS & & & ENT ELLIPSE & PARAMETERS & \\
\hline $\begin{array}{l}\text { LAT . } \\
\text { I,ONG. }\end{array}$ & $\begin{array}{l}\text { LENGTH } \\
\text { (DAYS) }\end{array}$ & $\begin{array}{l}\text { DEPTH } \\
\text { (M) }\end{array}$ & $\begin{array}{l}\text { ВотTOM } \\
\text { (14) }\end{array}$ & $\begin{array}{c}\text { EAST } \\
(\mathrm{CM} / \mathrm{SEC})\end{array}$ & $\begin{array}{c}\text { PHASE } \\
(\mathrm{DEG}-\mathrm{G})\end{array}$ & $\begin{array}{c}\text { NORTH } \\
(\mathrm{CM} / \mathrm{SEC})\end{array}$ & $\begin{array}{r}\text { PHASE } \\
(\text { DEG-G) }\end{array}$ & $\begin{array}{r}\text { UMAJOR } \\
\text { (CM/SEC) }\end{array}$ & $\begin{array}{l}\text { UMINOR } \\
\text { (CM/SEC) }\end{array}$ & $\begin{array}{r}\text { PHASE } \\
(\text { DEG -G) }\end{array}$ & $\begin{array}{c}\text { ORIEN } \\
\text { (DEG-TRUE) }\end{array}$ \\
\hline
\end{tabular}

GREAT SOUTH CHANNEL

\begin{tabular}{|c|c|c|c|c|c|c|c|c|c|c|c|c|c|c|}
\hline $\begin{array}{l}\text { M } \\
40^{\circ} 51-\mathrm{N} . \\
68^{\circ} 49-\mathrm{W} .\end{array}$ & $\begin{array}{l}145 \\
171 \\
116 \\
124\end{array}$ & $\begin{array}{l}10-\mathrm{H} \\
10-\mathrm{R} \\
51-\mathrm{H} \\
51-\mathrm{R}\end{array}$ & $\begin{array}{l}56 \\
56 \\
15 \\
15\end{array}$ & $\begin{array}{l}4 \cdot 1+-0.3 \\
2.3+-0.1 \\
3.1+-0.2 \\
2.6+-0.1\end{array}$ & $\begin{array}{l}140+- \\
199+- \\
168+- \\
239+-\end{array}$ & $\begin{array}{r}12 \\
3 \\
5 \\
2\end{array}$ & $\begin{array}{r}11.8+-0.8 \\
8.2+-0.2 \\
10.0+-0.9 \\
6.2+-0.1\end{array}$ & $\begin{array}{r}62+- \\
118+- \\
64+- \\
108+-\end{array}$ & $\begin{array}{l}6 \\
2 \\
4 \\
1\end{array}$ & $\begin{array}{l}11.9+-0.8 \\
8.2 \\
10.0+-0.9 \\
6.5\end{array}$ & $\begin{array}{l}-3.9+-0.3 \\
-2.3 \\
-3.0+-0.2 \\
-1.9\end{array}$ & $\begin{array}{l}64+- \\
119 \\
62+- \\
103\end{array}$ & 4 & $\begin{array}{l}5+- \\
3 \\
355+- \\
343\end{array}$ \\
\hline $\begin{array}{l}40^{\circ} 49^{-} \mathrm{N} . \\
69^{\circ} 00^{-} \mathrm{W} .\end{array}$ & 87 & 58 & 20 & $2.6+-0.7$ & $155+-$ & 20 & $9.5+-0.7$ & $64+-$ & 4 & $9.5+-0.7$ & $-2.6+-0.6$ & $65+-$ & 3 & $360+-$ \\
\hline $\begin{array}{l}\mathrm{GSC} 2 \\
40^{\circ} 51^{-} \mathrm{N} . \\
69^{\circ} 01^{-} \mathrm{W} .\end{array}$ & $\begin{array}{l}109 \\
152 \\
152\end{array}$ & $\begin{array}{l}10 \\
42 \\
76\end{array}$ & $\begin{array}{r}73 \\
41 \\
7\end{array}$ & $\begin{array}{l}4.1+-0.3 \\
2.2+-0.2 \\
2.7+-0.4\end{array}$ & $\begin{array}{l}173+- \\
210+- \\
258+-\end{array}$ & $\begin{array}{l}5 \\
3 \\
9\end{array}$ & $\begin{array}{l}9.7+-0.2 \\
8.9+-0.2 \\
4.8+-0.7\end{array}$ & $\begin{array}{r}98+- \\
113+- \\
162+-\end{array}$ & $\begin{array}{l}2 \\
2 \\
8\end{array}$ & $\begin{array}{l}9.8 \\
8.9 \\
4.8\end{array}$ & $\begin{array}{l}-3.9 \\
-2.2 \\
-2.7\end{array}$ & $\begin{array}{l}101 \\
113 \\
159\end{array}$ & & $\begin{array}{r}8 \\
358 \\
355\end{array}$ \\
\hline $\begin{array}{l}40^{\circ} 51^{-} \mathrm{N} . \\
69^{\circ} 01^{-} \mathrm{W} .\end{array}$ & 145 & 68 & 15 & $3.6+-0.4$ & $123+-$ & 9 & $10.0+-0.7$ & $66+-$ & 3 & $10.2+-0.6$ & $-3.0+-0.5$ & $70+-$ & 4 & $12+-$ \\
\hline $\begin{array}{l}\mathrm{R} \\
40^{\circ} 30^{-} \mathrm{N} \\
69^{\circ} 07^{-} \mathrm{W} .\end{array}$ & 116 & 79 & 1 & $3.3+-0.4$ & $109+-$ & 23 & $4.5+-0.7$ & $56+-$ & 7 & $5.0+-0.5$ & $-2.3+-0.9$ & $70+-$ & 6 & $29+-$ \\
\hline $\begin{array}{l}\mathrm{GSC} 1 \\
40^{\circ} 52^{-} \mathrm{N} .\end{array}$ & $\begin{array}{l}149 \\
149\end{array}$ & $\begin{array}{l}27 \\
49\end{array}$ & $\begin{array}{l}37 \\
15\end{array}$ & $\begin{array}{l}2.3+-0.1 \\
2.7+-0.1\end{array}$ & $\begin{array}{l}165+- \\
138+-\end{array}$ & $\begin{array}{l}2 \\
2\end{array}$ & $\begin{array}{l}8.0+-0.1 \\
7.0+-0.1\end{array}$ & $\begin{array}{l}117+- \\
113+-\end{array}$ & $\begin{array}{l}1 \\
1\end{array}$ & $\begin{array}{l}8.2 \\
7.4\end{array}$ & $\begin{array}{l}-1.7 \\
-1.1\end{array}$ & $\begin{array}{l}119 \\
116\end{array}$ & & $\begin{array}{l}11 \\
20\end{array}$ \\
\hline
\end{tabular}

LYDONIA CANYON*

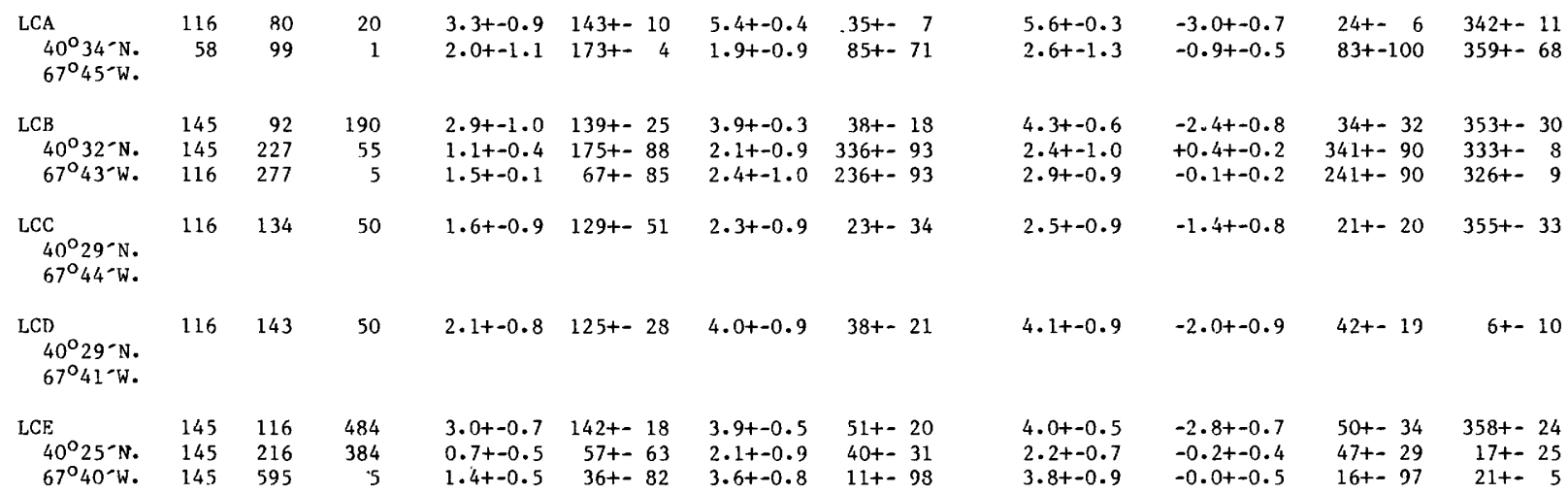

Instrument type, method of analysis and source of data are listed alphabetically by stations in Table 3 .

If UMINOR < 0 , ellipse rotates clockwise

$\mathrm{H}=$ harmonic $\quad \mathrm{R}=$ response

* - Observations from station LCB(except 277m), LCE, LCH(except 1375 and 1454m), LCI, LCJ(except 454m), and LCN al1 begin at 0100 on Dec.2, 1980 and end at 0100 on Apri1 26, 1981. Observations at LCA(80m), LCB $(277 \mathrm{~m}), \mathrm{LCJ}(83 \mathrm{~m}), \mathrm{LCK}(454 \mathrm{~m})$ and LCI begin at 0100 on Dec. 2, 1980 but end 29 days earlier on March 28,1981. 
Table 7. $\mathrm{S}_{2}$ tidal current parameters-Continued

Table 7. S2 TIDAL CURRENT PARAMETERS--Continued

$\begin{array}{cccc} & \text { FOURIER } & \text { COEFFICIENTS } \\ \text { EAST } & \text { PHASE } & \text { NORTH } & \text { PHASE: } \\ (\mathrm{CM} / \mathrm{SEC}) & (\mathrm{DEG}-\mathrm{G}) & (\mathrm{CM} / \mathrm{SEC}) & (\mathrm{DEG}-\mathrm{G})\end{array}$

$\begin{array}{cccc}\text { CURRENT } & \text { ELLIPSE } & \text { PARAMETERS } \\ \text { UMAJOR } & \text { UMINOR } & \text { PHASE } & \text { ORIEN } \\ \text { (CM/SEC) } & (\text { CM/SEC) } & \text { (DEG-G) } & \text { (DEG-TRUE) }\end{array}$

$\begin{array}{cl}\text { STATION } & \text { RECORD INSTR ABOVE } \\ \text { LAT. } & \text { LENGTH DEPTH BOTTOM }\end{array}$

$\begin{array}{ll}\text { LAT. } & \text { LENGTH DEPTH BOTTOM } \\ \text { LONG. } & \text { (DAYS) (M) (M) }\end{array}$

LYDONIA CANYON--CONT.

$\begin{array}{lrrr}\text { LCF } & 145 & 205 & 300 \\ 40^{\circ} 21^{-N} . & 174 & 405 & 100 \\ 67^{\circ} 39^{-} \mathrm{W} . & & & \\ & & & \\ \text { LCG } & 174 & 195 & 300 \\ 40^{\circ} 211^{\circ} \mathrm{N} . & 174 & 395 & 100 \\ 67^{\circ} 42^{-} \mathrm{W} . & & & \\ \text { LCH }^{*} & 145 & 290 & 1264 \\ 40^{\circ} 18^{\circ} \mathrm{N} . & 145 & 540 & 1014 \\ 67^{\circ} 40^{-} \mathrm{W} . & 145 & 890 & 664 \\ & 29 & 1454 & 100 \\ & 87 & 1375 & 5\end{array}$

$\begin{array}{llll}\text { LCI } & 145 & 10 & 240\end{array}$

$40^{\circ} 23^{-N}$ N. $145 \quad 55 \quad 195$

$67^{\circ} 33^{-}$- $.145 \quad 195 \quad 55$

$\begin{array}{llll}\text { LCJ } & 116 & 83 & 488\end{array}$

$40^{\circ} 21^{-N} . \quad 145 \quad 223 \quad 348$

$67^{\circ} 32^{-}$-W. $145 \quad 471 \quad 100$

LCK $\quad 145 \quad 204 \quad 350$

$67^{\circ} 47^{-} \mathrm{W}$.

LCL $\quad 116 \quad 65 \quad 60$

$40^{\circ} 32^{-\mathrm{N}} . \quad 116 \quad 105 \quad 20$

$67^{\circ} 36^{-\mathrm{W}}$.

LCM $^{* *} \quad 145 \quad 103 \quad 20$

$40^{\circ} 30^{-} \mathrm{N}$.
$67^{\circ} 49^{-} \mathrm{W}$.

$\begin{array}{llll}\text { LCN } & 145 & 243 & 798 \\ 40^{\circ} 21^{-} \text {N. } & 145 & 841 & 200\end{array}$

$67^{\circ} 40^{-} \mathrm{W}$. $40^{\circ} 16^{-} \mathrm{N} . \quad 145 \quad 454 \quad 100$

$\begin{array}{lccccccc}1.8+-1.0 & 117+-39 & 2.0+-0.6 & 41+-23 & 2.3+-0.8 & -1.4+-0.9 & 51+-37 & 13+-50 \\ 1.5+-0.9 & 38+-79 & 1.0+-0.7 & 47+-64 & 1.8+-1.1 & -0.1+-0.2 & 38+-40 & 49+-60 \\ & & & & & & & \\ 1.9+-0.8 & 93+-90 & 2.0+-1.2 & 12+-53 & 2.5+-1.1 & -1.1+-0.8 & 17+-68 & 22+-45 \\ 0.6+-0.3 & 98+-69 & 0.7+-0.4 & 31+-76 & 0.9+-0.5 & -0.3+-0.1 & 33+-85 & 27+-50 \\ & & & & & & & \\ 1.3+-0.3 & 292+-100 & 1.3+-0.6 & 83+-82 & 1.5+-0.4 & -0.9+-0.7 & 99+-92 & 316+-19 \\ 0.7+-0.3 & 82+-103 & 1.2+-0.4 & 76+-102 & 1.3+-0.4 & -0.4+-0.3 & 86+-97 & 15+-26 \\ 0.6+-0.3 & 186+-63 & 0.9+-0.6 & 64+-55 & 1.1+-0.7 & -0.1+-0.2 & 58+-43 & 348+-46 \\ 0.6 & 68 & 2.1 & 48 & 2.1 & -0.2 & 49 & 15 \\ 0.2+-0.2 & 128+-30 & 1.2+-0.8 & 57+-91 & 1.2+-0.7 & -0.2+-0.1 & 1+-99 & 21+-38 \\ & & & & & & & \\ 2.2+-0.9 & 174+-103 & 2.7+-1.4 & 41+-25 & 3.3+-0.9 & -1.0+-1.5 & 30+-44 & 356+-49 \\ 1.8+-0.9 & 163+-50 & 1.9+-0.8 & 55+-34 & 2.2+-0.8 & -1.4+-0.9 & 40+-48 & 328+-33 \\ 2.4+-1.4 & 100+-32 & 1.6+-0.9 & 23+-73 & 2.4+-1.4 & -1.3+-1.2 & 74+-73 & 65+-69 \\ 1.0+-0.7 & 200+-100 & 1.6+-1.1 & 113+-83 & 1.7+-1.0 & -0.8+-0.7 & 178+-99 & 350+-57 \\ & & & & & & & \\ 1.7+-0.5 & 152+-61 & 2.1+-0.9 & 38+-34 & 2.4+-0.5 & -1.0+-1.0 & 30+-64 & 328+-34 \\ 1.1+-0.6 & 62+-79 & 1.1+-0.3 & 316+-63 & 1.2+-0.4 & -0.6+-0.8 & 328+-46 & 20+-60 \\ 0.7+-0.3 & 239+-105 & 0.6+-0.3 & 286+-70 & 0.8+-0.2 & -0.2+-0.5 & 289+-59 & 340+-66 \\ 1.0+-0.4 & 345+-90 & 1.1+-0.5 & 116+-91 & 1.5+-0.3 & -0.5+-0.4 & 89+-84 & 330+-45 \\ 1.1+-0.7 & 118+-71 & 1.3+-0.4 & 342+-31 & 1.6+-0.6 & -0.6+-0.7 & 325+-22 & 331+-24 \\ & & & & & & & \\ 3.8+-0.7 & 162+-24 & 4.6+-1.2 & 55+-12 & 5.1+-0.9 & -3.1+-0.7 & 56+-48 & 352+-58 \\ 3.9+-0.9 & 131+-13 & 4.1+-1.1 & 29+-12 & 4.8+-1.0 & -3.0+-0.6 & 19+-51 & 343+-52 \\ & & & & & & & \\ 3.0+-1.8 & 141+-74 & 3.5+-1.2 & 35+-27 & 3.7+-1.5 & -2.5+-1.9 & 31+-44 & 361+-31 \\ 1.5+-1.1 & 103+-74 & 2.1+-0.4 & 29+-37 & 2.3+-0.8 & -1.1+-1.0 & 20+-46 & 356+-30 \\ & & & & & & & \\ 1.8+-0.8 & 90+-84 & 1.9+-0.5 & 354+-66 & 2.3+-0.7 & -1.1+-0.8 & 28+-81 & 33+-28 \\ 0.8+-0.4 & 64+-58 & 1.1+-0.7 & 19+-58 & 1.4+-0.8 & -1.6+-0.3 & 21+-59 & 25+-27\end{array}$

NANTUCKET SHOALS

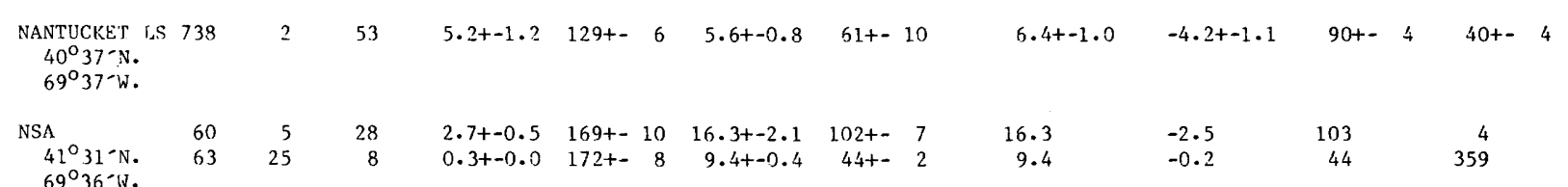

Instrument type, method of analysis and source of data are listed alphabetically by stations in Table 3. If UMINOR < 0 , ellipse rotates clockwise

* - two separate moorings at different depths.

**- tripod and subsurface mooring were at different depths 
Table 7. $S_{2}$ tidal current parameters-Continued

Table 7. S2 TIDAL CURRENT PARAMETERS--Continued

\begin{tabular}{|c|c|c|c|c|c|c|}
\hline $.0 \mathrm{~N}$ & DRD & INSTR & ABOVE & & OURIER & COEFEICIENTS \\
\hline $\begin{array}{l}\text { LAT. } \\
\text { LONG. }\end{array}$ & $\begin{array}{l}\text { LENGTH } \\
\text { (DAYS) }\end{array}$ & $\begin{array}{c}\text { DEPTH } \\
\text { (M) }\end{array}$ & $\begin{array}{l}\text { BOTTOM } \\
\text { (M) }\end{array}$ & $\begin{array}{c}\text { EAST } \\
(\mathrm{CM} / \mathrm{SEC})\end{array}$ & $\begin{array}{c}\text { PHASE } \\
(\mathrm{DEG}-\mathrm{G})\end{array}$ & $\begin{array}{c}\text { NORTH } \\
(\mathrm{CM} / \mathrm{SEC})\end{array}$ \\
\hline
\end{tabular}

\begin{tabular}{cccc}
\multicolumn{2}{c}{ CURRENT ELLLPSE } & PARAMETERS \\
UMAJOR & UMINOR & PHASE & ORIEN \\
$(\mathrm{CM} / \mathrm{SEC})$ & $(\mathrm{CM} / \mathrm{SEC})$ & (DEG-G) & (DEG-TRUE)
\end{tabular}

NANTUCKET SHOALS--CONT.

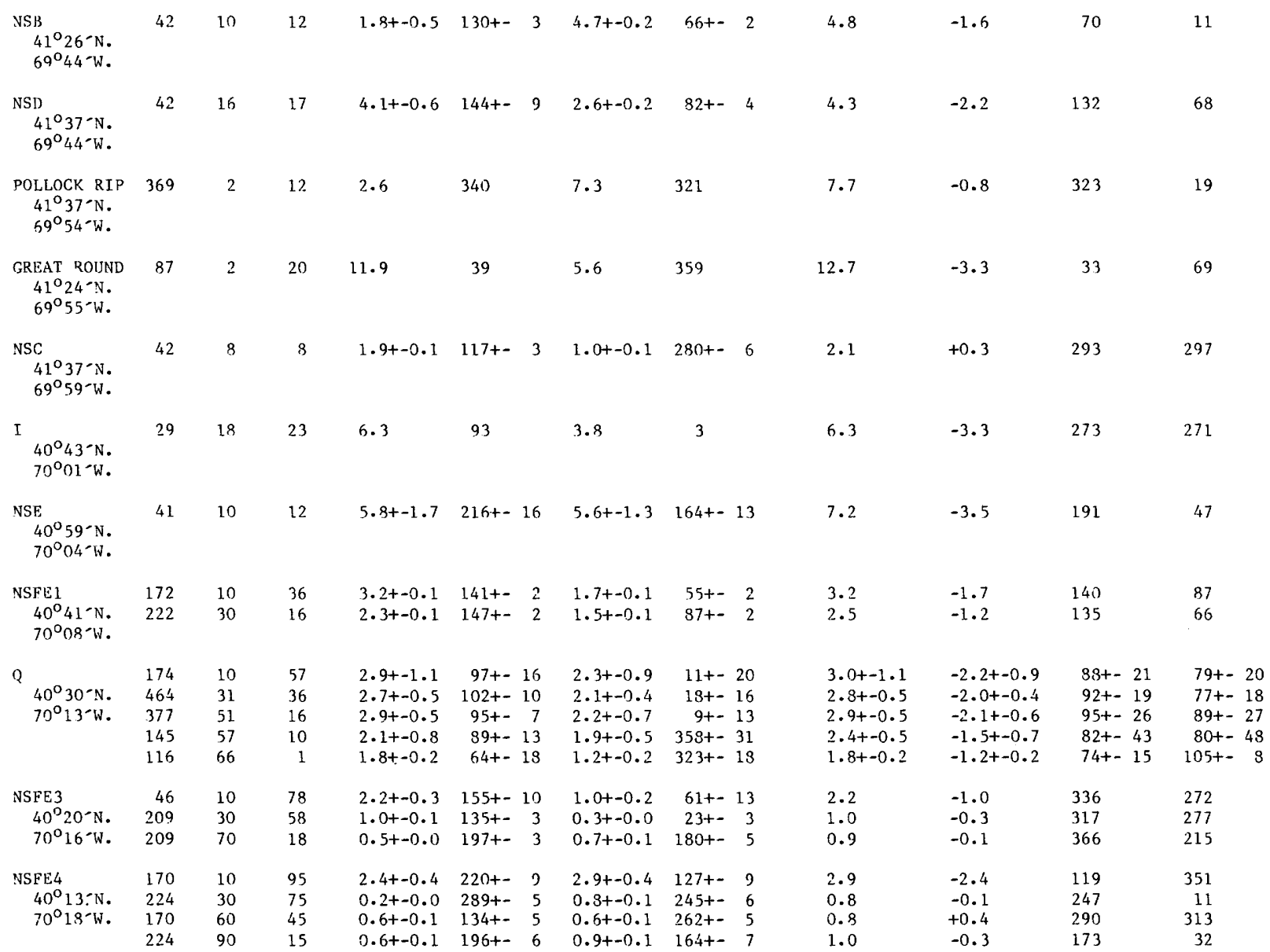

Instrument type, method of analysis and source of data are listed alphabetically by stations in Table 3 .

If UMINOR $<0$, ellipse rotates clockwise 
Table 7. $\mathrm{S}_{2}$ tidal current parameters-Continued

Table 7. S2 TIDAL CURRENT PARAMETERS--Continued

STATION RECORD INSTR ABOVE

LAT. LENGTH DEPTH BOTTOM

LONG. (DAYS) (M) (M)

NANTUCKET SHOALS--CONT.

$\begin{array}{lrrr}\text { NSFE5 } & 171 & 10 & 188 \\ 40^{\circ} 02^{\circ} \text { N. } & 171 & 30 & 168 \\ 70^{\circ} 22^{-W} . & 171 & 90 & 108 \\ & 171 & 120 & 78 \\ & 171 & 185 & 13 \\ \text { NSFE6 } & 225 & 10 & 800 \\ 39^{\circ} 51^{\circ} \mathrm{N} . & & & \\ 70^{\circ} 25^{\circ} \mathrm{W} . & & & \end{array}$

COEFF IC IENTS

NORTH PHASE

$(\mathrm{CM} / \mathrm{SEC}) \quad(\mathrm{DEG}-\mathrm{G}) \quad(\mathrm{CM} / \mathrm{SEC}) \quad(\mathrm{DEG}-\mathrm{G})$

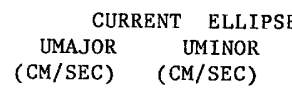

(CM/SEC)
PARAMETERS

PHASE - ORIEN (DEG-G) (DEG-TRUE)
W ENGLAND SHELF

$40^{\circ} 29^{-N} . \quad 87 \quad 61 \quad 10$

$70^{\circ} 30^{-} \mathrm{w}$.

NES743

$\begin{array}{llll}35 & 20 & 85\end{array}$

$70^{\circ} 52^{\prime} \mathrm{W}$.

$\begin{array}{llll}\text { NES764 } & 180 \quad 305 & 1995\end{array}$

$39^{\circ} 37^{\prime} \mathrm{N} . \quad 180 \quad 2005 \quad 295$

$70^{\circ} 56-\mathrm{W}$.

$\begin{array}{llll}\text { NES742 } & 35 & 20 & 54\end{array}$

$40^{\circ} 35^{\circ} \mathrm{N} . \quad 35 \quad 60 \quad 14$

$70^{\circ} 59^{\prime}$ W.

HENS\&CHICK. 369

$41^{\circ} 27^{-} \mathrm{N}$.

$71^{\circ} 01^{-W}$.

NES763

$39^{\circ} 56^{\circ} \mathrm{N}$.

$71^{\circ} 03^{-W}$.

$\operatorname{Nas762}$

$40^{\circ} 28^{-N}$

NES 741 $40^{\circ} 56^{-N}$. $71^{\circ} 13-\mathrm{W}$.

$1.1+-0.5 \quad 141+-26$

$0.7 \quad 132$

$0.5+-0.2138+-19$

$0.6+-0.1203+-12$

$0.6+-0.2 \quad 289+-17$

$1.0+-0.5331+-26$

0.8

318

$0.7+-0.3100+-24$

$1.1+-0.3 \quad 130+-15$

$1.4+-0.4 \quad 225+-17$

$1.1+-0.6118+-33$

$0.8+-0.4351+-26$

1.5

1.1

1.4

1.2

$-0.1$

$-0.1$

-0.6
-0.5

$-0.6$

326
315
113
138
229
313

312

313

15

12

301
$1.7+-0.5 \quad 85+-14 \quad 1.3+-0.5 \quad 338+-18$ $0.8+-0.8 \quad 354+-70 \quad 1.1+-0.3 \quad 272+-58$

$1.9+-0.5$

$1 \cdot 1+-0.3$

$-1 \cdot 2+-0.6$

$260+-43 \quad 271+-38$

$1.0+-0.1 \quad 72+-8 \quad 1.4+-0.2 \quad 344+-10$

1.4

$-1.0$

$0.1+-0.0 \quad 64+-7 \quad 0.4+-0.1 \quad 342+-9$

0.4

$-0.1$

346

343

2

$0.6+-0.2 \quad 186+-20 \quad 0.5+-0.5 \quad 93+-54$

$0.3+-0.1 \quad 343+-19 \quad 0.5+-0.4 \quad 248+-49$

0.6

0.5

$-0.5$

$-0.3$

13

245

278

$0.9+-0.0 \quad 96+-\quad 4 \quad 0.4+-0.0 \quad 275+-5$

1.0

-0.0
-0.4

276

276

294

283

$5 \cdot 1$

$-1.6$

323

71

0.8

$-0.1$

266

6

$1.1+-0.1 \quad 74+-4 \quad 1.6+-0.1 \quad 304+-5$

$1 \cdot 8$
1.2

$-0.8$

-0.8
-0.4

291

270

330

1.2

$-0.7$

111

70

.475

5.4

308

5.4

$-0.4$

308

357 $-0.3+-0.8$ $41^{\circ} 26^{\prime} \mathrm{N}$. $71^{\circ} 23-\mathrm{w}$.

Instrument type, method of analysis and source of data are 1isted alphabetically by stations in Table 3 .

If UMINOR < 0 , ellipse rotates clockwise

* - Orientation is questionable 
Table 7. $\mathrm{S}_{2}$ tidal current parameters-Continued

Table 7. S2 TIDAL CURRENT PARAMETERS--Continued

STATION RECORD INSTR ABOVE

LAT. LENGTH DEPTH BOTTOM

LONG. $\begin{array}{ccc} & \text { FOURIER } & \text { COEFFICIENTS } \\ \text { EAST } & \text { PHASE } & \text { NORTH }\end{array}$

$(\mathrm{CM} / \mathrm{SEC}) \quad(\mathrm{DEG}-\mathrm{G}) \quad(\mathrm{CM} / \mathrm{SEC}) \quad(\mathrm{DEG}-\mathrm{G})$

\begin{tabular}{cccc}
\multicolumn{2}{c}{ CURRENT } & ELLLPSE & PARAMETERS \\
UIMAJOR & UMINOR & PHASE & ORIEN \\
$(\mathrm{CM} / \mathrm{SEC})$ & $(\mathrm{CM} / \mathrm{SEC})$ & $($ DEG $-\mathrm{G})$ & (DEG-TRUE)
\end{tabular}

MIDDLE ATLANTIC BIGHT

\begin{tabular}{|c|c|c|c|c|c|c|c|c|c|c|c|c|c|}
\hline $\begin{array}{l}\text { NES763W } \\
39^{\circ} 43^{\circ} \mathrm{N} . \\
71^{\circ} 47^{-} \mathrm{W} .\end{array}$ & 183 & 302 & 202 & $0.5+-0.2$ & $302+-$ & 19 & $0.8+-0.3$ & $216+-$ & 24 & 0.8 & -0.6 & 221 & 7 \\
\hline $\begin{array}{l}\text { NES762W } \\
39^{\circ} 55^{-N} \text {. } \\
71^{\circ} 58^{-} \mathrm{W} .\end{array}$ & 172 & 38 & 45 & $1.4+-0.1$ & $61+-$ & 4 & $1.9+-0.2$ & $304+-$ & 5 & 2.1 & -1.1 & 288 & 332 \\
\hline $\begin{array}{l}\text { LT } 5 \\
40^{\circ} 12^{-\mathrm{N}} . \\
72^{\circ} \mathrm{O0}-\mathrm{W} .\end{array}$ & $\begin{array}{r}87 \\
70 \\
116 \\
70 \\
87 \\
70 \\
87 \\
70\end{array}$ & $\begin{array}{l}21-\mathrm{H} \\
21-\mathrm{R} \\
41-\mathrm{H} \\
41-\mathrm{R} \\
61-\mathrm{H} \\
61-\mathrm{R} \\
66-\mathrm{H} \\
66-\mathrm{R}\end{array}$ & $\begin{array}{r}46 \\
46 \\
26 \\
26 \\
6 \\
6 \\
1 \\
1\end{array}$ & $\begin{array}{l}2.3+-1.1 \\
2.8+-0.3 \\
1.9+-1.1 \\
1.4+-0.1 \\
1.7+-0.4 \\
0.3+-0.0 \\
1.0+-0.5 \\
0.6+-0.1\end{array}$ & $\begin{array}{r}74+- \\
74+- \\
77+- \\
122+- \\
52+- \\
68+- \\
39+- \\
59+-\end{array}$ & $\begin{array}{r}5 \\
6 \\
29 \\
6 \\
8 \\
5 \\
16 \\
6\end{array}$ & $\begin{array}{l}2.0+-0.9 \\
1.9+-0.3 \\
1.8+-1.2 \\
1.0+-0.1 \\
1.5+-0.2 \\
1.1+-0.2 \\
0.8+-0.2 \\
0.7+-0.1\end{array}$ & $\begin{array}{r}325+- \\
4+- \\
319+- \\
53+- \\
283+- \\
228+- \\
274+- \\
254+-\end{array}$ & $\begin{array}{r}18 \\
8 \\
12 \\
8 \\
17 \\
8 \\
8 \\
10\end{array}$ & $\begin{array}{l}2.5+-1.3 \\
2.9 \\
2.2+-1.2 \\
1.5 \\
2.1+-0.1 \\
1.1 \\
1.2+-0.5 \\
0.9\end{array}$ & $\begin{array}{l}-1.6+-0.6 \\
-1.7 \\
-1.5+-1.2 \\
-0.9 \\
-0.9+-0.2 \\
+0.1 \\
-0.6+-0.2 \\
-0.1\end{array}$ & $\begin{array}{ll}276+- & 5 \\
242 & \\
288+- & 27 \\
288 & \\
252+- & 2 \\
229 & \\
243+- & 5 \\
248 & \end{array}$ & $\begin{array}{l}301+- \\
250 \\
315+- \\
247 \\
309+- \\
346 \\
311+- \\
320\end{array}$ \\
\hline $\begin{array}{l}\text { LT4 } \\
\qquad 40^{\circ} 34^{-} \mathrm{N} \\
72^{\circ} 19^{-} \mathrm{W}\end{array}$ & $\begin{array}{r}29 \\
203 \\
174 \\
87\end{array}$ & $\begin{array}{r}3 \\
24 \\
44 \\
51\end{array}$ & $\begin{array}{r}49 \\
28 \\
8 \\
1\end{array}$ & $\begin{array}{l}2.0+-0.9 \\
3.5+-1.2 \\
2.5+-0.5 \\
1.5+-0.6\end{array}$ & $\begin{array}{l}33+- \\
60+- \\
72+- \\
80+-\end{array}$ & $\begin{array}{r}59 \\
34 \\
24 \\
2\end{array}$ & $\begin{array}{l}1.0+-0.0 \\
2.2+-1.0 \\
1.5+-0.5 \\
0.3+-0.8\end{array}$ & $\begin{array}{l}271+- \\
315+- \\
320+- \\
313+-\end{array}$ & $\begin{array}{l}75 \\
34 \\
19 \\
39\end{array}$ & $\begin{array}{l}2.1+-0.8 \\
3.7+-1.2 \\
2.6+-0.5 \\
1.5+-0.6\end{array}$ & $\begin{array}{l}-0.8+-0.2 \\
-1.8+-1.1 \\
-1.3+-0.4 \\
-0.7+-0.9\end{array}$ & $\begin{array}{l}222+-50 \\
247+-34 \\
260+-28 \\
265+-\quad 4\end{array}$ & $\begin{array}{l}292+- \\
283+- \\
287+- \\
281+-\end{array}$ \\
\hline $\begin{array}{l}\text { CMICE } \\
\quad 40^{\circ} 47-\mathrm{N} . \\
72^{\circ} 49-\mathrm{W} .\end{array}$ & $\begin{array}{l}25 \\
25 \\
25 \\
25\end{array}$ & $\begin{array}{r}4 \\
8 \\
16 \\
25\end{array}$ & $\begin{array}{r}25 \\
21 \\
13 \\
4\end{array}$ & $\begin{array}{l}1.2+-0.1 \\
1.3+-0.1 \\
1.1+-0.1 \\
0.4+-0.0\end{array}$ & $\begin{array}{r}76+- \\
94+- \\
85+- \\
100+-\end{array}$ & $\begin{array}{l}3 \\
6 \\
5 \\
6\end{array}$ & $\begin{array}{l}0.7+-0.4 \\
0.5+-0.3 \\
0.7+-0.4 \\
0.3+-0.2\end{array}$ & $\begin{array}{r}312+- \\
319+- \\
315+- \\
44+-\end{array}$ & $\begin{array}{l}31 \\
29 \\
14 \\
27\end{array}$ & $\begin{array}{l}1.3 \\
1.4 \\
1.2 \\
0.4\end{array}$ & $\begin{array}{l}-0.5 \\
-0.4 \\
-0.5 \\
-0.2\end{array}$ & $\begin{array}{l}266 \\
280 \\
277 \\
263\end{array}$ & $\begin{array}{l}292 \\
290 \\
297 \\
239\end{array}$ \\
\hline $\begin{array}{l}\mathrm{ME} \\
39^{\circ} 57^{-N} \text {. } \\
72^{\circ} 36^{-} \mathrm{N} .\end{array}$ & 29 & 59 & 1 & 1.1 & 56 & & 1.5 & 286 & & 1.7 & -0.7 & 271 & 328 \\
\hline $\begin{array}{l}\mathrm{NJ} 4 \\
38^{\circ} 55^{-\mathrm{N}} . \\
72^{\circ} 58^{-} \mathrm{W} .\end{array}$ & $\begin{array}{l}72 \\
72 \\
72\end{array}$ & $\begin{array}{r}3 \\
43 \\
91\end{array}$ & $\begin{array}{r}89 \\
49 \\
1\end{array}$ & $\begin{array}{l}2.2+-0.6 \\
1.3+-0.2 \\
0.8+-0.3\end{array}$ & $\begin{array}{r}71+- \\
166+- \\
30+-\end{array}$ & $\begin{array}{r}14 \\
7 \\
24\end{array}$ & $\begin{array}{l}3.0+-0.8 \\
2.9+-0.5 \\
0.3+-0.2\end{array}$ & $\begin{array}{r}315+- \\
22+- \\
259+-\end{array}$ & $\begin{array}{l}16 \\
11 \\
33\end{array}$ & $\begin{array}{l}3.2 \\
3.1 \\
0.8\end{array}$ & $\begin{array}{l}-1.8 \\
-0.7 \\
-0.2\end{array}$ & $\begin{array}{r}299 \\
17 \\
214\end{array}$ & $\begin{array}{l}333 \\
339 \\
285\end{array}$ \\
\hline $\begin{array}{l}\text { IIA } \\
39^{\circ} 27^{-} \mathrm{N} . \\
73^{\circ} 00^{-} \mathrm{W} .\end{array}$ & 58 & 58 & 1 & $1.5+-0.3$ & $56+-$ & 50 & $1.7+-0.8$ & $286+-$ & 17 & $2.0+-0.5$ & $-1.0+-0.8$ & $270+-18$ & $324+-$ \\
\hline $\begin{array}{l}\text { LT3 } \\
\qquad 39^{\circ} 16^{-} \mathrm{N} \\
73^{\circ} 02^{-} \mathrm{W}\end{array}$ & $\begin{array}{r}104 \\
104 \\
70\end{array}$ & $\begin{array}{r}9 \\
19 \\
58\end{array}$ & $\begin{array}{l}61 \\
51 \\
12\end{array}$ & $\begin{array}{l}1.8+-0.1 \\
1.7+-0.2 \\
4.2+-0.4\end{array}$ & $\begin{array}{r}123+- \\
124+- \\
69+-\end{array}$ & $\begin{array}{l}5 \\
6 \\
6\end{array}$ & $\begin{array}{l}1.4+-0.1 \\
1.6+-0.2 \\
3.5+-0.4\end{array}$ & $\begin{array}{l}331+- \\
337+- \\
310+-\end{array}$ & $\begin{array}{l}6 \\
6 \\
7\end{array}$ & $\begin{array}{l}2.2 \\
2.2 \\
4.8\end{array}$ & $\begin{array}{l}-0.5 \\
-0.7 \\
-2.7\end{array}$ & $\begin{array}{l}313 \\
319 \\
271\end{array}$ & $\begin{array}{l}307 \\
313 \\
305\end{array}$ \\
\hline $\begin{array}{l}\text { UESA7 } \\
\quad 39^{\circ} 55^{-} \mathrm{N} . \\
73^{\circ} 06^{-\mathrm{W}} .\end{array}$ & $\begin{array}{r}107 \\
103 \\
63\end{array}$ & $\begin{array}{l}18 \\
38 \\
66\end{array}$ & $\begin{array}{r}50 a \\
30 a \\
2 a\end{array}$ & $\begin{array}{l}2.7+-0.3 \\
1.6+-0.2 \\
0.2+-0.0\end{array}$ & $\begin{array}{l}135+- \\
111+- \\
157+-\end{array}$ & $\begin{array}{r}6 \\
5 \\
12\end{array}$ & $\begin{array}{l}2.2+-0.3 \\
2.1+-0.3 \\
0.9+-0.1\end{array}$ & $\begin{array}{l}310+- \\
348+- \\
275+-\end{array}$ & $\begin{array}{l}8 \\
8 \\
8\end{array}$ & $\begin{array}{l}3.5 \\
2.4 \\
0.9\end{array}$ & $\begin{array}{l}+0.1 \\
-1.2 \\
+0.2\end{array}$ & $\begin{array}{l}313 \\
331 \\
276\end{array}$ & $\begin{array}{l}309 \\
328 \\
354\end{array}$ \\
\hline $\begin{array}{l}\text { FIRE IS. } \\
40^{\circ} 29^{\circ} \mathrm{N} .\end{array}$ & 369 & 2 & 27 & 1.4 & 114 & & 0.6 & 349 & & 1.4 & -0.5 & 299 & 286 \\
\hline
\end{tabular}
$73^{\circ} 11-\mathrm{W}$.

Instrument type, method of analysis and source of data are 1 isted alphabetically by stations in Table 3 . If UMINOR < 0 , ellipse rotates clockwise

$H$ - harmonic analysis $R$ - response analysis

a - water depth taken from chart 13200 
Table 7. $S_{2}$ tidal current parameters-Continued

Table 7. S2 TIDAL CURRENT PARAMETERS--Continued

\begin{tabular}{clllcccr} 
STATION & RECORD INSTR ABOVE & \multicolumn{3}{c}{ FOURIER } & COEFFICIENTS \\
LAT. & LENGTH & DEPTH & BOTTOM & EAST & PHASE & NORTH & PHASE \\
LONG. & (DAYS) & (M) & (M) & $(\mathrm{CM} / \mathrm{SEC})$ & $($ DEG $-G)$ & $(\mathrm{CM} / \mathrm{SEC})$ & $(\mathrm{DEG}-\mathrm{G})$
\end{tabular}

$\begin{array}{cccc}\text { CURRENT ELLIPSE } & \text { PARAMETERS } \\ \text { UMAJOR } & \text { UMINOR } & \text { PHASE } & \text { ORIEN } \\ \text { (CM/SEC) } & (\text { CM/SEC) } & \text { (DEG-G) } & \text { (DEG-TRUE) }\end{array}$

MIDDLE ATLANTIC BIGHT--CONT.

\begin{tabular}{|c|c|c|c|c|c|c|c|c|c|c|c|}
\hline $\begin{array}{l}\mathrm{MF} \\
38^{\circ} 31^{-} \mathrm{N} . \\
73^{\circ} 14^{-\mathrm{W}} .\end{array}$ & $\begin{array}{l}116 \\
116\end{array}$ & $\begin{array}{r}15 \\
232\end{array}$ & $\begin{array}{r}219 \\
2\end{array}$ & $\begin{array}{l}2.1+-0.5 \\
0.6+-0.1\end{array}$ & $\begin{array}{r}146+-89 \\
11+-52\end{array}$ & $\begin{array}{l}1.4+-0.9 \\
0.7+-0.4\end{array}$ & $\begin{array}{l}353+-54 \\
167+-66\end{array}$ & $\begin{array}{l}2.4+-0.3 \\
0.9+-0.3\end{array}$ & $\begin{array}{l}-0.8+-0.8 \\
+0.1+-0.4\end{array}$ & $\begin{array}{l}318+-61 \\
180+-42\end{array}$ & $\begin{array}{l}345+-70 \\
322+-31\end{array}$ \\
\hline $\begin{array}{l}\mathrm{MC} \\
38^{\circ} 33^{-} \mathrm{N} . \\
73^{\circ} 31^{-\mathrm{W}} .\end{array}$ & 29 & 79 & 1 & 1.1 & 51 & 1.1 & 263 & 1.4 & -0.4 & 247 & 315 \\
\hline $\begin{array}{l}\mathrm{MB} \\
38^{\circ} 44^{-} \mathrm{N} . \\
73^{\circ} 38^{-} \mathrm{W} .\end{array}$ & $\begin{array}{r}406 \\
58 \\
522 \\
58 \\
87\end{array}$ & $\begin{array}{l}15 \\
45 \\
50 \\
54 \\
59\end{array}$ & $\begin{array}{r}45 \\
15 \\
10 \\
6 \\
1\end{array}$ & $\begin{array}{l}2.8+-0.6 \\
2.9+-1.0 \\
2.2+-0.6 \\
2.8+-0.8 \\
1.2+-0.3\end{array}$ & $\begin{array}{lr}99+- & 23 \\
87+- & 9 \\
82+- & 14 \\
83+- & 2 \\
74+- & 29\end{array}$ & $\begin{array}{l}2.7+-0.6 \\
2.7+-0.6 \\
2.3+-0.8 \\
2.4+-0.5 \\
1.5+-0.5\end{array}$ & $\begin{array}{rr}341+- & 23 \\
333+- & 20 \\
320+- & 15 \\
324+- & 9 \\
305+- & 9\end{array}$ & $\begin{array}{l}3.4+-0.6 \\
3.3+-0.8 \\
2.8+-0.7 \\
3.2+-0.7 \\
1.8+-0.5\end{array}$ & $\begin{array}{l}-1.9+-0.5 \\
-2.2+-0.9 \\
-1.5+-0.6 \\
-1.8+-0.7 \\
-0.7+-0.3\end{array}$ & $\begin{array}{l}309+-20 \\
295+-\quad 3 \\
292+-10 \\
285+-5 \\
288+-20\end{array}$ & $\begin{array}{lr}313+-13 \\
310+-12 \\
317+-12 \\
306+- & 9 \\
325+- & 13\end{array}$ \\
\hline $\begin{array}{l}\mathrm{LT} 2 \\
39^{\circ} 24^{-} \mathrm{N} . \\
73^{\circ} 43^{-\mathrm{W}} .\end{array}$ & $\begin{array}{l}116-c \\
232\end{array}$ & $\begin{array}{r}3 \\
15\end{array}$ & $\begin{array}{l}31 \\
19\end{array}$ & $\begin{array}{l}2.7+-0.6 \\
3.3+-0.7\end{array}$ & $\begin{array}{l}91+-12 \\
89+-15\end{array}$ & $\begin{array}{l}3.0+-0.9 \\
2.9+-0.6\end{array}$ & $\begin{array}{l}330+-10 \\
336+-26\end{array}$ & $\begin{array}{l}3.5+-0.9 \\
3.9+-0.5\end{array}$ & $\begin{array}{l}-2.0+-0.6 \\
-2.2+-0.4\end{array}$ & $\begin{array}{l}303+-13 \\
293+-23\end{array}$ & $\begin{array}{l}319+-\quad 6 \\
306+-25\end{array}$ \\
\hline $\begin{array}{l}\text { BARNEGAT } \\
39^{\circ} 46^{-} \mathrm{N} . \\
73^{\circ} 56^{-} \mathrm{W} .\end{array}$ & 369 & 2 & 22 & 0.4 & 98 & 0.7 & 322 & 0.7 & -0.2 & 313 & 335 \\
\hline $\begin{array}{l}\text { MD } \\
38^{\circ} 59^{-} \mathrm{N} . \\
74^{\circ} 02^{-} \mathrm{W} .\end{array}$ & 116 & 40 & 1 & $1.2+-0.3$ & $73+-25$ & $1.4+-0.3$ & $312+-31$ & $1.6+-0.3$ & $-0.9+-0.3$ & $289+-23$ & $323+-$ \\
\hline $\begin{array}{c}\text { L. EGG INLET } \\
39^{\circ} 28^{-N} \text {. } \\
74^{\circ} 15^{-} \mathrm{W} .\end{array}$ & $\begin{array}{l}264 \\
365\end{array}$ & $\begin{array}{r}5 \\
10\end{array}$ & $\begin{array}{l}7 \\
2\end{array}$ & $\begin{array}{l}1.3 \\
0.8\end{array}$ & $\begin{array}{l}126 \\
113\end{array}$ & $\begin{array}{l}1.2 \\
0.7\end{array}$ & $\begin{array}{l}360 \\
327\end{array}$ & $\begin{array}{l}1.6 \\
1.0\end{array}$ & $\begin{array}{l}-0.8 \\
-0.3\end{array}$ & $\begin{array}{l}331 \\
308\end{array}$ & $\begin{array}{l}312 \\
310\end{array}$ \\
\hline $\begin{array}{c}\text { NE END L.S. } \\
38^{\circ} 58^{-N} \text {. }\end{array}$ & 369 & 2 & 2,4 & 1.7 & 130 & 1.0 & 331 & 2.0 & -0.3 & 315 & 298 \\
\hline
\end{tabular}

SOUTHERN MIDDLE ATLANTIC B BghT

\begin{tabular}{|c|c|c|c|c|c|c|c|c|c|c|c|c|}
\hline $\begin{array}{l}\text { WINTER QU } \\
37^{\circ} 55^{-} \mathrm{N} . \\
74^{\circ} 56^{-\mathrm{W}} .\end{array}$ & 369 & 2 & 22. & 0.3 & 139 & & 0.3 & 3 & 0.4 & -0.2 & 336 & 308 \\
\hline $\begin{array}{l}\text { MAB } \\
36^{\circ} 50^{-} \mathrm{N} . \\
75^{\circ} 02^{-} \mathrm{W} .\end{array}$ & $\begin{array}{l}53 \\
52 \\
53\end{array}$ & $\begin{array}{r}9 \\
21 \\
32\end{array}$ & $\begin{array}{r}29 \\
17 \\
6\end{array}$ & $\begin{array}{l}2.4+-0.2 \\
1.7+-0.2 \\
3.4+-0.4\end{array}$ & $\begin{array}{l}126+- \\
179+- \\
158+-\end{array}$ & $\begin{array}{l}5 \\
7 \\
7\end{array}$ & $\begin{array}{l}1.2+-0.2 \\
1.9+-0.2 \\
3.8+-0.7\end{array}$ & $\begin{array}{rr}340+- & 11 \\
71+- & 6 \\
354+- & 11\end{array}$ & $\begin{array}{l}2.6 \\
2.1 \\
5.1\end{array}$ & $\begin{array}{l}-0.6 \\
-1.5 \\
-0.7\end{array}$ & $\begin{array}{r}312 \\
44 \\
347\end{array}$ & $\begin{array}{l}294 \\
325 \\
318\end{array}$ \\
\hline $\begin{array}{l}\text { DIAMOND } \\
35^{\circ} 05^{\circ} \mathrm{N} . \\
75^{\circ} 20^{-} \mathrm{W} .\end{array}$ & 738 & 2 & 51 & $0.3+-0.1$ & $16+-$ & 8 & $0.8+-0.0$ & $318+-88$ & $0.8+-0.1$ & $-0.1+-0.1$ & $318+-89$ & $2+-13$ \\
\hline $\begin{array}{l}\text { CHESAPEARF, } \\
36^{\circ} 59^{-} \mathrm{N} \text {. }\end{array}$ & 369 & 2 & 17 & 1.8 & 201 & & 0.3 & 63 & 1.9 & -0.2 & 22 & 277 \\
\hline
\end{tabular}
$36^{\circ} 59^{-N} \mathrm{~N}$. $75^{\circ} 42-\mathrm{W}$.

Instrument type, method of analysis and source of data are listed alphabetically by stations in Table 3 .

If UMINOR < 0 , ellipse rotates clockwise

$c$ - average of 4 records $31,31,32$ and 29 meters above bottom 
Table 7. $\mathrm{S}_{2}$ tidal current parameters-Continued

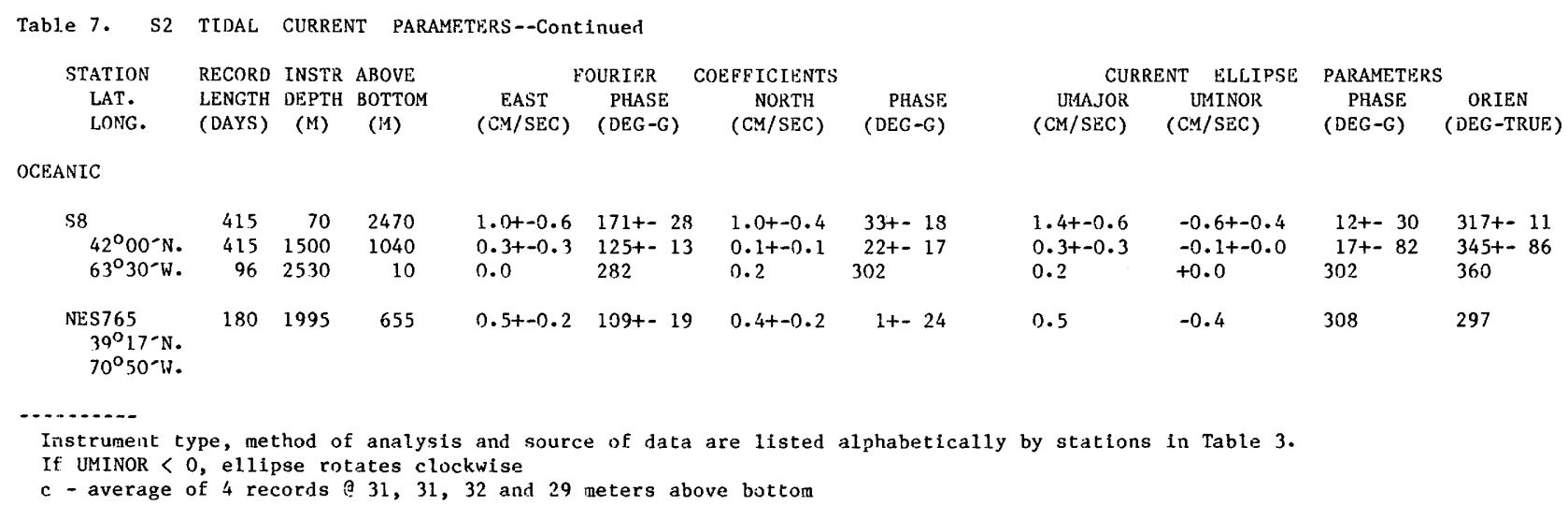


Table 8. $K_{1}$ tidal current parameters (see table 5 for explanation).

Table 8. K1 TIDAL CURRENT PARAMETERS

\begin{tabular}{|c|c|c|c|c|c|c|c|c|c|c|c|}
\hline TAT & RECORD & INSTR & ABOVE & & OURIER & COEFFICIENTS & & $\mathrm{CU}$ & ELLIP & PARAMF & \\
\hline $\begin{array}{l}\text { LAT. } \\
\text { LONG. }\end{array}$ & $\begin{array}{l}\text { LENGTH } \\
\text { (DAYS) }\end{array}$ & $\begin{array}{l}\text { DEPTH } \\
(M)\end{array}$ & $\begin{array}{l}\text { BOTTOM } \\
\text { (M) }\end{array}$ & $\begin{array}{c}\text { EAST } \\
(\mathrm{CM} / \mathrm{SEC})\end{array}$ & $\begin{array}{r}\text { PHASE } \\
(\mathrm{DEG}-\mathrm{G})\end{array}$ & $\begin{array}{r}\text { NORTH } \\
(\mathrm{CM} / \mathrm{SEC})\end{array}$ & $\begin{array}{l}\text { PHASE } \\
(D E G-G)\end{array}$ & $\begin{array}{l}\text { UMAJOR } \\
\text { (CM/SEC) }\end{array}$ & $\begin{array}{l}\text { UMINOR } \\
\text { (CM/SEC) }\end{array}$ & $\begin{array}{c}\text { PHASE } \\
(D E G-G)\end{array}$ & $\begin{array}{c}\text { ORIEN } \\
\text { (DEQ-TRUE) }\end{array}$ \\
\hline
\end{tabular}

SCOTIAN SHELF

\begin{tabular}{|c|c|c|c|c|c|c|c|c|c|c|c|}
\hline $\begin{array}{l}\text { SS } 10 \\
43^{\circ} 34^{-N} . \\
59^{\circ} 04^{-W} .\end{array}$ & $\begin{array}{l}32 \\
22 \\
12\end{array}$ & $\begin{array}{r}200 \\
500 \\
1500\end{array}$ & $\begin{array}{r}1400 \\
1100 \\
100\end{array}$ & $\begin{array}{l}0.2 \\
0.4 \\
1.6\end{array}$ & $\begin{array}{l}189 \\
253 \\
208\end{array}$ & $\begin{array}{l}0.2 \\
0.8 \\
1.8\end{array}$ & $\begin{array}{l}210 \\
272 \\
127\end{array}$ & $\begin{array}{l}0.3 \\
0.9 \\
1.9\end{array}$ & $\begin{array}{l}+0.1 \\
+0.1 \\
-1.5\end{array}$ & $\begin{array}{l}200 \\
268 \\
149\end{array}$ & $\begin{array}{l}45 \\
26 \\
27\end{array}$ \\
\hline $\begin{array}{l}\text { SS2A } \\
\quad 42^{\circ} 52^{-\mathrm{N}} . \\
62^{\circ} 00^{-} \mathrm{W} .\end{array}$ & 35 & 555 & 245 & 0.2 & 338 & 0.5 & 160 & 0.5 & -0.0 & 160 & 338 \\
\hline $\begin{array}{l}\text { SS8 } \\
\quad 42^{\circ} 37^{\circ} \mathrm{N} . \\
62^{\circ} 05^{\circ} \mathrm{W} .\end{array}$ & $\begin{array}{r}5 \\
36\end{array}$ & $\begin{array}{r}200 \\
1500\end{array}$ & $\begin{array}{r}1350 \\
50\end{array}$ & $\begin{array}{l}1.5 \\
0.3\end{array}$ & $\begin{array}{r}289 \\
19\end{array}$ & $\begin{array}{l}1.3 \\
0.3\end{array}$ & $\begin{array}{r}150 \\
14\end{array}$ & $\begin{array}{l}1.9 \\
0.4\end{array}$ & $\begin{array}{l}-0.7 \\
-0.0\end{array}$ & $\begin{array}{r}126 \\
17\end{array}$ & $\begin{array}{r}310 \\
45\end{array}$ \\
\hline $\begin{array}{l}\text { SSIA } \\
\quad 42^{\circ} 52^{-} \mathrm{N} . \\
62^{\circ} 14^{-} \mathrm{W} .\end{array}$ & $\begin{array}{l}35 \\
35\end{array}$ & $\begin{array}{l}290 \\
490\end{array}$ & $\begin{array}{r}260 \\
60\end{array}$ & $\begin{array}{l}0.8 \\
0.4\end{array}$ & $\begin{array}{l}266 \\
332\end{array}$ & $\begin{array}{l}0.9 \\
0.2\end{array}$ & $\begin{array}{l}137 \\
155\end{array}$ & $\begin{array}{l}1.1 \\
0.4\end{array}$ & $\begin{array}{l}-0.5 \\
-0.0\end{array}$ & $\begin{array}{l}116 \\
153\end{array}$ & $\begin{array}{l}320 \\
297\end{array}$ \\
\hline $\begin{array}{l}\text { SS3 } \\
\quad 43^{\circ} 22^{-} \mathrm{N} . \\
62^{\circ} 40^{-} \mathrm{W} .\end{array}$ & $\begin{array}{r}238 \\
149 \\
47 \\
15 \\
136\end{array}$ & $\begin{array}{l}20 \\
50 \\
81 \\
91 \\
95\end{array}$ & $\begin{array}{r}79 \\
49 \\
18 \\
8 \\
4\end{array}$ & $\begin{array}{l}7.4+-2.2 \\
7.9+-2.7 \\
5.1 \\
7.1 \\
4.2+-1.7\end{array}$ & $\begin{array}{l}270+-7 \\
247+-7 \\
300 \\
285 \\
282+-13\end{array}$ & $\begin{array}{l}5.0+-1.8 \\
5.5+-2.1 \\
4.6 \\
6.1 \\
4.0+-1.5\end{array}$ & $\begin{array}{l}190+-11 \\
171+-10 \\
238 \\
229 \\
228+-15\end{array}$ & $\begin{array}{l}7.6+-2.2 \\
8 \cdot 1+-2.7 \\
5.9 \\
8.3 \\
5.2+-1.9\end{array}$ & $\begin{array}{l}-4 \cdot 8+-1 \cdot 8 \\
-5 \cdot 2+-2 \cdot 0 \\
-3 \cdot 5 \\
-4 \cdot 3 \\
-2 \cdot 7+-1 \cdot 1\end{array}$ & $\begin{array}{l}255+-74 \\
236+-8 \\
275 \\
263 \\
257+-11\end{array}$ & $\begin{array}{lr}68+- & 90 \\
74+- & 2 \\
51 & \\
53 & \\
48+- & 4\end{array}$ \\
\hline $\begin{array}{l}\text { SS7 } \\
\quad 43^{\circ} 02^{-} \mathrm{N} . \\
62^{\circ} 54^{-} \mathrm{W} .\end{array}$ & $\begin{array}{l}27 \\
27\end{array}$ & $\begin{array}{r}50 \\
118\end{array}$ & $\begin{array}{r}75 \\
7\end{array}$ & $\begin{array}{l}7.4 \\
4.4\end{array}$ & $\begin{array}{l}270 \\
308\end{array}$ & $\begin{array}{l}4.4 \\
4.3\end{array}$ & $\begin{array}{l}198 \\
243\end{array}$ & $\begin{array}{l}7.6 \\
5.2\end{array}$ & $\begin{array}{l}-4.1 \\
-3.3\end{array}$ & $\begin{array}{l}262 \\
277\end{array}$ & $\begin{array}{l}75 \\
47\end{array}$ \\
\hline $\begin{array}{l}\mathrm{SS} 2 \\
\quad 43^{\circ} 45^{-\mathrm{N}} \\
62^{\circ} 59^{-\mathrm{W}}\end{array}$ & $\begin{array}{l}189 \\
283 \\
166 \\
272\end{array}$ & $\begin{array}{r}20 \\
50 \\
95 \\
250\end{array}$ & $\begin{array}{r}258 \\
228 \\
183 \\
28\end{array}$ & $\begin{array}{l}5.4+-1.2 \\
5.5+-1.7 \\
3.9+-1.2 \\
3.0+-1.0\end{array}$ & $\begin{array}{l}248+-46 \\
281+-6 \\
279+-10 \\
294+-13\end{array}$ & $\begin{array}{l}2.4+-1.2 \\
2.3+-0.6 \\
2.7+-0.5 \\
3.7+-1.0\end{array}$ & $\begin{array}{l}215+-54 \\
266+-13 \\
284+-14 \\
314+-13\end{array}$ & $\begin{array}{l}5.9+-1.5 \\
6.0+-1.8 \\
4.8+-1.2 \\
4.7+-1.4\end{array}$ & $\begin{array}{l}-0.9+-0.2 \\
-0.5+-0.4 \\
+0.2+-0.2 \\
+0.8+-0.4\end{array}$ & $\begin{array}{rr}245+- & 47 \\
280+- & 6 \\
281+- & 11 \\
306+- & 12\end{array}$ & $\begin{array}{lr}70+- & 10 \\
68+- & 6 \\
54+- & 7 \\
38+- & 4\end{array}$ \\
\hline $\begin{array}{l}\mathrm{SS} 6 \\
\quad 43^{\circ} 15^{-} \mathrm{N} . \\
63^{\circ} 22^{-} \mathrm{W} .\end{array}$ & $\begin{array}{l}26 \\
27\end{array}$ & $\begin{array}{r}50 \\
130\end{array}$ & $\begin{array}{r}85 \\
5\end{array}$ & $\begin{array}{l}4.4 \\
2.6\end{array}$ & $\begin{array}{l}312 \\
309\end{array}$ & $\begin{array}{l}2.0 \\
2.9\end{array}$ & $\begin{array}{l}241 \\
286\end{array}$ & $\begin{array}{l}4.5 \\
3.8\end{array}$ & $\begin{array}{l}-1.9 \\
-0.8\end{array}$ & $\begin{array}{l}308 \\
296\end{array}$ & $\begin{array}{l}80 \\
42\end{array}$ \\
\hline $\begin{array}{l}\text { SS1 } \\
\quad 44^{\circ} 26^{\circ} \mathrm{N} . \\
63^{\circ} 29^{-} \mathrm{W} .\end{array}$ & $\begin{array}{l}211 \\
187\end{array}$ & $\begin{array}{l}14 \\
95\end{array}$ & $\begin{array}{r}87 \\
6\end{array}$ & $\begin{array}{l}6.9+-3.0 \\
1.6+-0.3\end{array}$ & $\begin{array}{r}273+-79 \\
90+-27\end{array}$ & $\begin{array}{l}3.2+-1 \cdot 6 \\
5.0+-3 \cdot 5\end{array}$ & $\begin{array}{l}224+-79 \\
231+-93\end{array}$ & $\begin{array}{l}7.6+-2.9 \\
5.4+-3.0\end{array}$ & $\begin{array}{l}-0.9+-1.0 \\
+0.4+-0.2\end{array}$ & $\begin{array}{l}264+-83 \\
238+-97\end{array}$ & $\begin{array}{r}66+-12 \\
9+-40\end{array}$ \\
\hline $\begin{array}{l}\text { S5 } \\
42^{\circ} 30^{-} \mathrm{N} . \\
63^{\circ} 30^{-} \mathrm{W} .\end{array}$ & $\begin{array}{l}262 \\
262 \\
370\end{array}$ & $\begin{array}{r}.50 \\
150 \\
1534\end{array}$ & $\begin{array}{r}1470 \\
1370 \\
10\end{array}$ & $\begin{array}{l}1.3+-0.7 \\
0.9+-0.6 \\
0.3\end{array}$ & $\begin{array}{c}292+-23 \\
331+- \\
69\end{array}$ & $\begin{array}{l}1.4+-1.4 \\
0.8+-0.5 \\
0.3\end{array}$ & $\begin{array}{l}174+-41 \\
216+-\quad 4 \\
201\end{array}$ & $\begin{array}{l}1.7+-1.2 \\
1.1+-0.7 \\
0.4\end{array}$ & $\begin{array}{l}-0.9+-1.1 \\
-0.7+-0.5 \\
+0.2\end{array}$ & $\begin{array}{l}175+-62 \\
178+-5 \\
225\end{array}$ & $\begin{array}{l}340+-62 \\
309+-8 \\
315\end{array}$ \\
\hline
\end{tabular}

Instrument type, method of analysis and source of data are listed alphabetically by stations in Table 3 .

If UMINOR $<0$, ellipse rotates clockwise 
Table 8. $K_{1}$ tidal current parameters-Continued

Table 8. Kl TIDAL CURRENT PARAMETERS--Continued

\begin{tabular}{|c|c|c|c|c|c|c|c|c|c|c|c|}
\hline STATION & RECORD & INSTR & ABOVE & & DURIER & COEFFICIENTS & & CURR & ELLIPSE & PARAMETERS & \\
\hline $\begin{array}{l}\text { LAT. } \\
\text { LONG. }\end{array}$ & $\begin{array}{l}\text { LENGTH } \\
\text { (DAYS) }\end{array}$ & $\begin{array}{l}\text { DEPTH } \\
\text { (M) }\end{array}$ & $\begin{array}{l}\text { BOTTOM } \\
\text { (M) }\end{array}$ & $\begin{array}{c}\text { EAST } \\
(\mathrm{CM} / \mathrm{SEC})\end{array}$ & $\begin{array}{r}\text { PHASE } \\
\text { (DEG-G) }\end{array}$ & $\begin{array}{r}\text { NORTH } \\
(\mathrm{CM} / \mathrm{SEC})\end{array}$ & $\begin{array}{l}\text { PHASE } \\
(\text { DEG-G) }\end{array}$ & $\begin{array}{l}\text { UMAJOR } \\
\text { (CM/SEC) }\end{array}$ & $\begin{array}{l}\text { UMINOR } \\
\text { (CM/SEC) }\end{array}$ & $\begin{array}{l}\text { PHASE } \\
(\mathrm{DEG}-\mathrm{G})\end{array}$ & $\begin{array}{c}\text { ORIEN } \\
\text { (DEG-TRUE) }\end{array}$ \\
\hline
\end{tabular}

SCOTIAN SHELF--CONT.

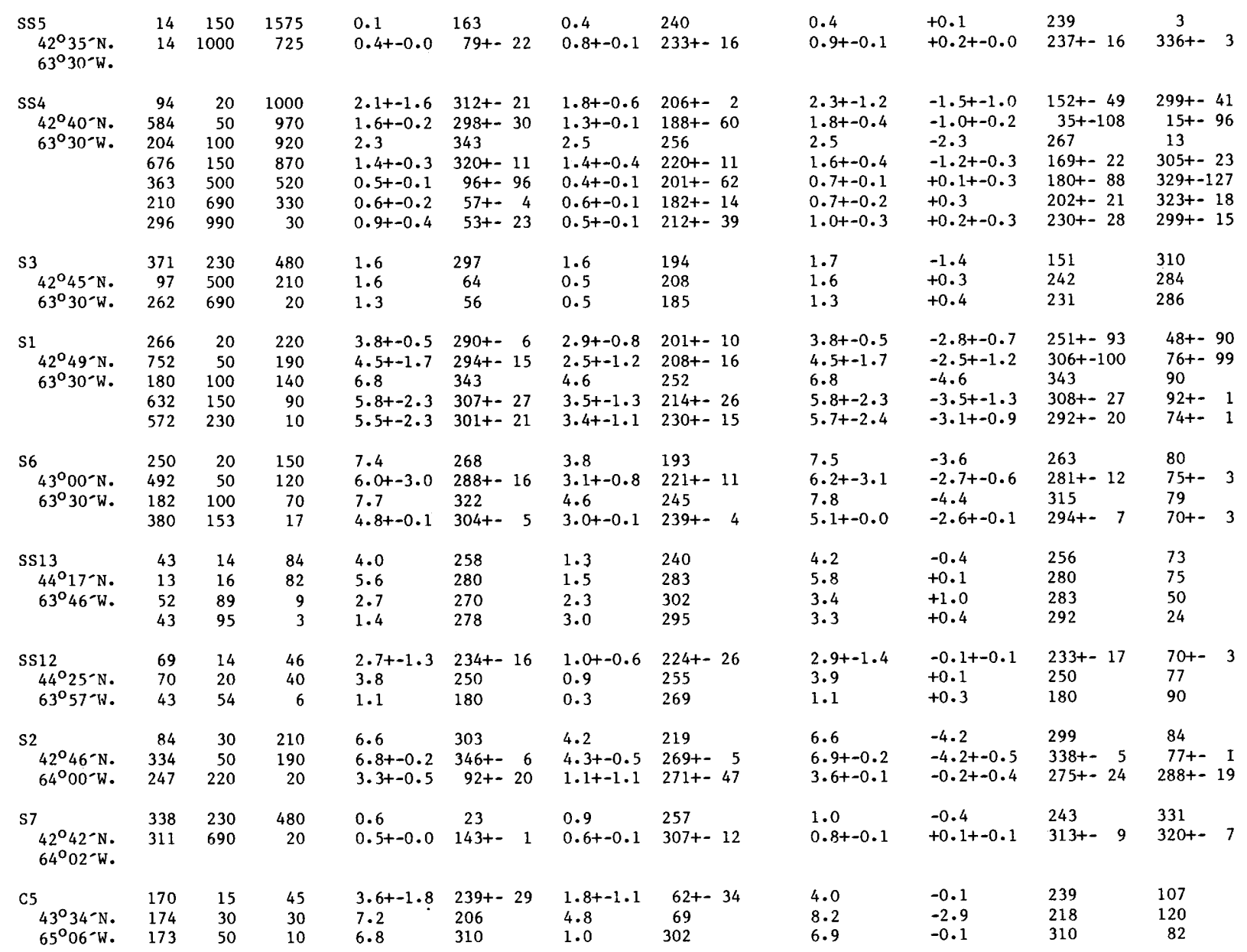

Instrument type, method of analysis and source of data are 1isted alphabetically by stations in Table 3 .

If IMINOR $<0$, ellipse rotates clockwise 
Table 8. $K_{1}$ tidal current parameters-Continued

Table 8. KI TIDAL CURREN PARAMETERS-Cont1nued

\begin{tabular}{|c|c|c|c|}
\hline $\begin{array}{c}\text { STATION } \\
\text { LAT. } \\
\text { LONG. }\end{array}$ & $\begin{array}{l}\text { RECORD } \\
\text { LENGTH } \\
\text { (DAYS) }\end{array}$ & $\begin{array}{c}\text { INSTI } \\
\text { DEPIt } \\
\text { (H) }\end{array}$ & $\begin{array}{l}\text { ABOVE } \\
\text { BOTIOM } \\
\text { (M) }\end{array}$ \\
\hline
\end{tabular}

\begin{tabular}{|c|c|c|c|}
\hline & OURIE & COEPPICIENTS & \\
\hline $\begin{array}{c}\text { BAST } \\
\text { CM/SEC) }\end{array}$ & $\begin{array}{l}\text { PHASE } \\
\text { (DEG-G) }\end{array}$ & $\begin{array}{r}\text { NORTH } \\
(\mathrm{CM} / \mathrm{SEC})\end{array}$ & $\begin{array}{l}\text { PHASE } \\
\text { (DEG-C) }\end{array}$ \\
\hline
\end{tabular}

\begin{tabular}{|c|c|c|c|}
\hline CURREN & NT ELLIPSE & PARAMETERS & \\
\hline $\begin{array}{l}\text { URAJOR } \\
\text { (CH/SEC) }\end{array}$ & $\begin{array}{l}\text { UMINOR } \\
\text { (CM/SEC) }\end{array}$ & $\begin{array}{l}\text { PHASE } \\
\text { (DEG-C) }\end{array}$ & $\begin{array}{c}\text { ORIEN } \\
\text { (DEG-TRUE) }\end{array}$ \\
\hline
\end{tabular}

SCOTIAN SHELE-CONT.

$\begin{array}{lrrr}\text { C1 } & 162 & 15 & 45 \\ 43^{\circ} 11^{\prime} \text { '. } & 162 & 30 & 30 \\ 65^{\circ} 43^{\prime} \text { '. } & 174 & 50 & 10 \\ & & & \\ \text { C3 } & 161 & 15 & 95 \\ 42^{\circ} 50^{\prime} \text { N. } & 103 & 50 & 60 \\ 65^{\circ} 50^{\prime} \text { W. } & 161 & 100 & 10\end{array}$

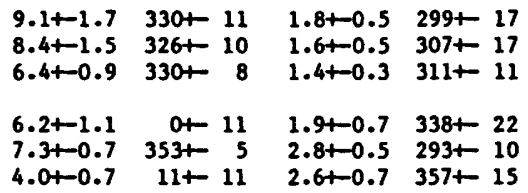

$\begin{array}{rrrr}9.3 & -0.9 & 329 & 80 \\ 8.5 & -0.5 & 326 & 80 \\ 6.6 & -0.5 & 329 & 78 \\ 6.5 & -0.7 & 358 & 74 \\ 7.5 & -2.4 & 349 & 78 \\ 4.8 & -0.5 & 7 & 57\end{array}$

NORTHEAST CHANNEL

$\begin{array}{lrrr}\text { NEC1 } & 174 & 103 & 120 \\ 42^{\circ} 22^{\prime} \mathrm{N} . & 174 & 153 & 70 \\ 65^{\circ} 56^{\prime} \mathrm{W} . & 174 & 207 & 16 \\ & & & \\ \text { NEC2* } & 58 & 106 & 134 \\ 42^{\circ} 18^{\prime} \mathrm{N} . & 174 & 156 & 84 \\ 65^{\circ} 58 \text { 'W. } & 58 & 217 & 17 \\ & & & \\ \text { NEC } & 87 & 112 & 116 \\ 42^{\circ} 11^{\circ} \mathrm{N} . & 87 & 162 & 66 \\ 66^{\circ} 02^{\prime} \mathrm{W} . & 174 & 220 & 16\end{array}$

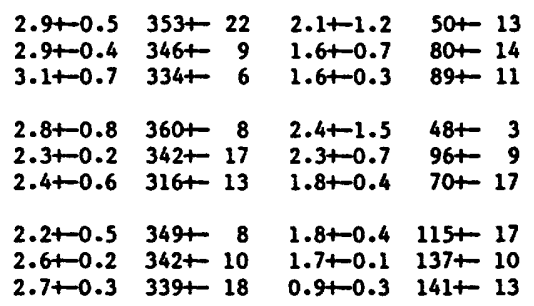

$2.7+0.3 \quad 339+-18 \quad 0.9+0.3 \quad 141+-13$

GULF OR MAINE

$\begin{array}{crrr}\text { CASHES LEDGE } & 58 & 33 & 1 \\ 43^{\circ} 11^{\prime} \mathrm{N} . & 58 & 68 & 1 \\ 69^{\circ} 05^{\prime} \mathrm{W} . & 58 & 180 & \\ & & & \\ \text { MONEGAN } & 57 & 33 \\ 43^{\circ} 40^{\prime} \mathrm{N} . & 58 & 68 \\ 69^{\circ} 23^{\prime} \mathrm{W} . & & \\ & & \\ \text { C.PORPOISE } & 74 & 33 \\ 43^{\circ} 13^{\prime} \mathrm{N} . & 74 & 68 \\ 70^{\circ} 17^{\prime} \mathrm{W} . & & \end{array}$

BOSTON L.S. $369 \quad 2$ $42^{\circ} 20^{\prime} \mathrm{N}$.

$70^{\circ} 45^{\prime} \mathrm{H}$.

BAY OF FUNDY

\begin{tabular}{|c|c|c|c|c|c|c|c|c|c|c|}
\hline $\begin{array}{l}\text { BED65 } \\
45^{\circ} 08^{\prime} \mathrm{H} . \\
65^{\circ} 08^{\prime} \mathrm{H} .\end{array}$ & $29 c$ & 25 & $37 a$ & 1.8 & 113 & 1.2 & 107 & 2.1 & -0.1 & 111 \\
\hline $\begin{array}{l}\text { BED66 } \\
45^{\circ} 25^{\prime} \mathrm{N} .\end{array}$ & $29 c$ & 25 & $13 a$ & 1.9 & 112 & 0.7 & 114 & 2.0 & +0.0 & 112 \\
\hline
\end{tabular}
$65^{\circ} 07^{\circ} \mathrm{W}$.

Inetrument type, nethod of analysis and source of data are 11sted alphabetically by stations in Table 3.

If UMINOR < 0 , ellipse rotates clockwise

* - Data 18 fron two separate noorings at different tiees 
Table 8. $K_{1}$ tidal current parameters-Continued

Table 8. K1 TIDAL CURRENT PARAMETERS--Continued

STATION RECORD INSTR ABOVE

$\begin{array}{cl}\text { RECION } & \text { RECRD } \\ \text { LAT. } & \text { LENGTH } \\ \text { DEPTH BOTTOM }\end{array}$

$\begin{array}{ll}\text { LONG. } & \text { LENGTH DEPTH BOTTOM } \\ \text { (DAYS) } & \text { (M) }\end{array}$

GULF OF MAINE--CONT.

\begin{tabular}{|c|c|c|c|c|c|c|c|c|c|c|c|c|}
\hline $\begin{array}{l}\text { BED64 } \\
\quad 45^{\circ} 13^{-} \mathrm{N} \\
65^{\circ} 14^{-} \mathrm{W}\end{array}$ & $\begin{array}{l}29 c \\
29 c\end{array}$ & $\begin{array}{l}10 \\
25\end{array}$ & $\begin{array}{l}40 a \\
25 a\end{array}$ & $\begin{array}{l}2.1 \\
2.1\end{array}$ & $\begin{array}{l}122 \\
125\end{array}$ & $\begin{array}{l}1.5 \\
1.2\end{array}$ & $\begin{array}{l}139 \\
128\end{array}$ & $\begin{array}{l}2.5 \\
2.4\end{array}$ & $\begin{array}{l}+0.4 \\
+0.0\end{array}$ & $\begin{array}{l}128 \\
126\end{array}$ & & $\begin{array}{l}55 \\
59\end{array}$ \\
\hline $\begin{array}{l}\text { BED63 } \\
\quad 45^{\circ} 19^{-} \mathrm{N} \\
65^{\circ} 20^{-} \mathrm{W}\end{array}$ & $29 c$ & 25 & $25 a$ & 1.6 & 113 & 1.2 & 121 & 2.0 & +0.1 & 116 & & 54 \\
\hline $\begin{array}{l}\text { BED62 } \\
\quad 44^{\circ} 39^{-} \mathrm{N} \\
66^{\circ} 02^{\circ} \mathrm{W}\end{array}$ & $29 c$ & 13 & $77 \mathrm{~b}$ & 1.9 & 86 & 1.3 & 118 & 2.2 & +0.6 & 96 & & 58 \\
\hline $\begin{array}{l}\text { BED } 61 \\
44^{\circ} 49^{-} \mathrm{N} . \\
66^{\circ} 12^{-} \mathrm{W}\end{array}$ & $\begin{array}{l}29 c \\
29 c\end{array}$ & $\begin{array}{l}13 \\
50\end{array}$ & $\begin{array}{l}94 \mathrm{~b} \\
57 \mathrm{~b}\end{array}$ & $\begin{array}{l}1.5 \\
1.6\end{array}$ & $\begin{array}{l}101 \\
113\end{array}$ & $\begin{array}{l}0.7 \\
0.9\end{array}$ & $\begin{array}{l}132 \\
132\end{array}$ & $\begin{array}{l}1.6 \\
1.8\end{array}$ & $\begin{array}{l}+0.4 \\
+0.3\end{array}$ & $\begin{array}{l}107 \\
118\end{array}$ & & $\begin{array}{l}65 \\
62\end{array}$ \\
\hline $\begin{array}{l}\text { BED } 60 \\
45^{\circ} 00^{-} \mathrm{N} \\
66^{\circ} 24^{-} \mathrm{W}\end{array}$ & $29 c$ & 13 & $71 b$ & 0.7 & 142 & 0.2 & 317 & 0.7 & +0.0 & 142 & & 109 \\
\hline $\begin{array}{l}\mathrm{L} \\
41^{\circ} 42^{-} \mathrm{N} . \\
66^{\circ} 36^{-} \mathrm{W} .\end{array}$ & 145 & 51 & 15 & $3.8+-0.5$ & $155+-15$ & $5.7+-0.7$ & $84+-9$ & $5.9+-0.6$ & $-3.5+-0.6$ & $96+-$ & 8 & $20+-6$ \\
\hline $\begin{array}{l}\mathrm{P} 4 \\
42^{\circ} 12^{-} \mathrm{N} \\
66^{\circ} 41^{-} \mathrm{W}\end{array}$ & $\begin{array}{l}29 \\
29\end{array}$ & $\begin{array}{r}79 \\
129\end{array}$ & $\begin{array}{r}140 \\
90\end{array}$ & $\begin{array}{l}1.8 \\
2.6\end{array}$ & $\begin{array}{l}332 \\
326\end{array}$ & $\begin{array}{l}3.3 \\
3.0\end{array}$ & $\begin{array}{l}60 \\
36\end{array}$ & $\begin{array}{l}3.3 \\
3.3\end{array}$ & $\begin{array}{l}+1.8 \\
+2.2\end{array}$ & $\begin{array}{l}239 \\
193\end{array}$ & & $\begin{array}{l}182 \\
212\end{array}$ \\
\hline $\begin{array}{l}\mathrm{P5} \\
42^{\circ} 02^{-} \mathrm{N} . \\
66^{\circ} 41^{-\mathrm{W}} .\end{array}$ & $\begin{array}{l}29 \\
29\end{array}$ & $\begin{array}{l}19 \\
44\end{array}$ & $\begin{array}{l}52 \\
27\end{array}$ & $\begin{array}{l}2.4 \\
2.1\end{array}$ & $\begin{array}{l}118 \\
116\end{array}$ & $\begin{array}{l}8.9 \\
4.3\end{array}$ & $\begin{array}{l}51 \\
56\end{array}$ & $\begin{array}{l}9.0 \\
4.5\end{array}$ & $\begin{array}{l}-2.2 \\
-1.8\end{array}$ & $\begin{array}{l}232 \\
243\end{array}$ & & $\begin{array}{l}186 \\
197\end{array}$ \\
\hline $\begin{array}{l}\text { P6 } \\
\quad 41^{\circ} 53^{-\mathrm{N}} . \\
66^{\circ} 41^{-\mathrm{W}}\end{array}$ & $\begin{array}{l}58 \\
58 \\
58\end{array}$ & $\begin{array}{l}11 \\
26 \\
36\end{array}$ & $\begin{array}{l}59 \\
44 \\
34\end{array}$ & $\begin{array}{l}3.8+-0.6 \\
3.3+-0.6 \\
3.0+-0.4\end{array}$ & $\begin{array}{l}126+-12 \\
133+-10 \\
152+-5\end{array}$ & $\begin{array}{l}6.7+-0.1 \\
6.0+-0.0 \\
5.2+-0.1\end{array}$ & $\begin{array}{ll}55+- & 1 \\
64+- & 2 \\
82+- & 3\end{array}$ & $\begin{array}{l}6.9+-0.3 \\
6.2+-0.2 \\
5.4+-0.3\end{array}$ & $\begin{array}{l}-3.4+-0.2 \\
-3.0+-0.2 \\
-2.7+-0.2\end{array}$ & $\begin{array}{l}242+- \\
252+- \\
270+-\end{array}$ & $\begin{array}{l}6 \\
7 \\
7\end{array}$ & $\begin{array}{l}194+-10 \\
195+-10 \\
196+-8\end{array}$ \\
\hline $\begin{array}{l}\mathrm{M}^{*} \\
40^{\circ} 56^{-\mathrm{N}} . \\
66^{\circ} 58^{-\mathrm{W}} .\end{array}$ & $\begin{array}{l}58 \\
58 \\
58\end{array}$ & $\begin{array}{l}10 \\
36 \\
69\end{array}$ & $\begin{array}{r}67 \\
41 \\
8\end{array}$ & $\begin{array}{l}4.9 \\
4.2 \\
2.8\end{array}$ & $\begin{array}{l}150 \\
168 \\
198\end{array}$ & $\begin{array}{l}3.4 \\
3.4 \\
3.1\end{array}$ & $\begin{array}{r}79 \\
80 \\
125\end{array}$ & $\begin{array}{l}5.1 \\
4.2 \\
3.4\end{array}$ & $\begin{array}{l}-3.1 \\
-3.4 \\
-2.5\end{array}$ & $\begin{array}{l}137 \\
164 \\
153\end{array}$ & & $\begin{array}{l}70 \\
86 \\
36\end{array}$ \\
\hline
\end{tabular}

Instrument type, method of analysis and source of data are 1isted alphabetically by stations in Table 3 .

If UMINOR < 0, ellipse rotates clockwise

a-water depth estimated from DMA chart 14040 (old 609)

b-water depth estimated from DMA chart 13102

0 -estimated to be at least 29-days

* - depths have been calculated from the mean pressure and error estimates are taken from across-isobath and along-isobath directions since these are nearly the same as the ellipse axis. These records span identical time periods. 
Table 8. $K_{1}$ tidal current parameters-Continued

Table 8. KI TIDAL CURRENT PARAMETERS-Cont1 nued

\begin{tabular}{|c|c|c|c|}
\hline $\begin{array}{l}\text { STATIOA } \\
\text { LAT. } \\
\text { LONG. }\end{array}$ & $\begin{array}{l}\text { RECORD } \\
\text { LENGTH } \\
\text { (DAYS) }\end{array}$ & $\begin{array}{l}\text { INST } \\
\text { DEPT } \\
\text { (M) }\end{array}$ & $\begin{array}{l}\text { ABOVE } \\
\text { BOTTOA } \\
\text { (M) }\end{array}$ \\
\hline
\end{tabular}

GEORGES BANK-CONT.<smiles>[R12]CCC</smiles>
$42^{\circ} 03^{\prime} \mathrm{N} . \quad 30 \quad 14$ $67^{\circ} 15^{\prime} \mathrm{W}$.

P3 $41^{\circ} 53^{\circ} \mathrm{N}$
$67^{\circ} 15^{\circ} \mathrm{W}$

$13^{*}$ $41^{\circ} 20^{\prime} \mathrm{N}$ $67^{\circ} 16^{\circ} \mathrm{H}$.

M9* $40^{\circ} 54^{\prime} \mathrm{K}$ $67^{\circ} 24^{\prime} \mathrm{H}$.

A $40^{\circ} 51^{\prime} \mathrm{N} . \quad 261 \quad 15$ $67^{\circ} 24^{\prime} \mathrm{W} .957 \quad 75$

c $41^{\circ} 24^{\prime} \mathrm{N}$. $67^{\circ} 34^{\prime} \mathrm{H}$.

$\mathbf{x}^{\text {*t }}$ $\begin{array}{rrr}58 & 10 & 54\end{array}$ N. $174 \quad 15 \quad 45$ $67^{\circ} 34^{\prime} \mathrm{H}$.

$\begin{array}{rr}58 & 34 \\ 174 & 54 \\ 87 & 58 \\ 232 & 60\end{array}$

D $41^{\circ} 59^{\prime} \mathrm{B}$ $87 \quad 15 \quad 69$ $67^{\circ} 47^{\prime}$ '.

GREAT SOUTH CHANKEL $\begin{array}{llll}40^{\circ} 51 ' N . & 145 & 10-\mathrm{B} & 56 \\ 68^{\circ} 49^{\prime} \mathrm{W} . & 171 & 10-\mathrm{R} & 56 \\ & 116 & 51-\mathrm{H} & 15 \\ & 124 & 51-\mathrm{R} & 15\end{array}$ $40^{\circ} 49^{\prime} \mathrm{H}$. $87 \quad 58 \quad 20$

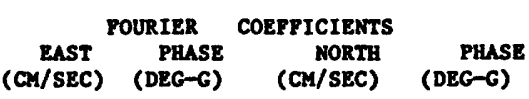

(AM/SBC)

$\begin{array}{cccc}\text { CURRENT ELLIPSE } & \text { PARAMETERS } \\ \text { UMAJOR } & \text { UMINOR } & \text { PHASE } & \text { ORIEN } \\ (\mathrm{CI} / \mathrm{SEC}) & \text { (CM/SEC) } & \text { (DEG-G) } & \text { (DEG-TRUE) }\end{array}$

$5.1+0$

$-2.4+-0.5$

199t 1 189t 3

3.7

25

3.9

$-2.1$

217

202

5.0

$-2.5 \quad 212$

$\begin{array}{ll}5.2 & -2.3 \\ 2.1 & +0.4\end{array}$

$212 \quad 205$

$\begin{array}{rr}4.6 & 19 \\ 4.9 & 32 \\ 0.7 & 310\end{array}$

$+0.4$

92

201

6.4

$-4.1$

110

49

3.0

$-1.7$

168

so

$4.8+-0.5 \quad-3.5+-0.8 \quad 155 t 29 \quad 77 t 33$

$4.6+0.7 \quad-3.4+0.6 \quad 144 t 17 \quad 61+17$

$3.1+0.6 \quad-2.0+0.6 \quad 157+21 \quad 50+17$

$2.3+-0.4 \quad-1.8+-0.4 \quad 142+18 \quad 53+12$

$\begin{array}{llll}4.3+0.7 & 168+12 & 3.7+-0.7 & 89+11\end{array}$

$\begin{array}{llll}2.7+0.6 & 186+14 & 2.5+-0.6 & 117 t \\ 2.1+-0.4 & 173+10 & 2.0+-0.4 & 96+11\end{array}$

$6.0+0.7$

$-3.7+0.5$

$93+943+4$

$6.2+0.5$

$-4.6+-0.2$

$142+$

$65+10$

$5.9+1.0 \quad-4.4 t-1.0 \quad 116 t-53 \quad 45+49$

$6.2+-0.5 \quad-4.6+-0.2 \quad 142+4 \quad 65+11$

$4.6+-0.8 \quad-3.3+-0.8 \quad 137+10 \quad 50+5$

$4.4+0.5 \quad-3.1+0.1 \quad 121+9$ sot 1

$3.2+-0.6 \quad-2.4 t-0.3 \quad 139+25 \quad 65 t-18$

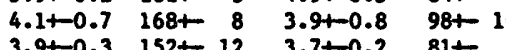

$3.0+0.6 \quad 158+19 \quad 2.6+-0.3 \quad 81+16$

$3.0+-0.2$

$71+1035+4$

$-1.3+-0.2$

$69^{\circ} 00^{\prime} \mathrm{H}$.

Instrunent type, wethod of analysis and source of data are 11sted alphabetically by stations in Table 3.

If $\mathrm{MIINOR}<0$, ell1pse rotates clocktise

A - harmonic analye1:

$R$ - response analyai:

- depths have been calculated from the sean preseure and error estiates are taken fron across-1sobath sad along-1sobath directions ince these are nearly the sane as the ellipse axis. These records span 1dentical tine periods.

th- tripod and subeurface noorings were at different depths 
Table 8. $K_{1}$ tidal current parameters-Continued

Table 8. K1 TIDAL CURRENT PARAMETERS--Continued

\begin{tabular}{|c|c|c|c|c|c|c|c|c|c|c|c|}
\hline CAT & RECORD & INSTR & ABOVE & & OURIER & COEFFICIENTS & & CURRENT & ELLIPSE & PARAMETERS & \\
\hline $\begin{array}{l}\text { LAT. } \\
\text { LONG. }\end{array}$ & $\begin{array}{l}\text { LENGTH } \\
\text { (DAYS) }\end{array}$ & $\begin{array}{c}\text { DEPTH } \\
(M)\end{array}$ & $\begin{array}{l}\text { BOTTOM } \\
\text { (M) }\end{array}$ & $\begin{array}{c}\text { EAST } \\
(\mathrm{CM} / \mathrm{SEC})\end{array}$ & $\begin{array}{r}\text { PHASE } \\
\text { (DEG-G) }\end{array}$ & $\begin{array}{r}\text { NORTH } \\
(\mathrm{CM} / \mathrm{SEC})\end{array}$ & $\begin{array}{l}\text { PHASE } \\
\text { (DEG-G) }\end{array}$ & $\begin{array}{l}\text { UMAJOR } \\
\text { (CM/SEC) }\end{array}$ & $\begin{array}{l}\text { UMINOR } \\
\text { CM/SEC) }\end{array}$ & $\begin{array}{l}\text { PHASE } \\
\text { (DEG-G) }\end{array}$ & $\begin{array}{c}\text { ORIEN } \\
\text { (DEG-TRUE) }\end{array}$ \\
\hline
\end{tabular}

CRF.AT SOUTH CHANNEL--CONT. $40^{\circ} 51-\mathrm{N} . \quad 152 \quad 42$ $69^{\circ} 01^{- \text {W. }} \quad 152 \quad 76$

$\mathrm{N}$ $40^{\circ} 51-\mathrm{N}$ $69^{\circ} 01^{\prime} \mathrm{W}$.

$\mathrm{R}$ $40^{\circ} 30^{-} \mathrm{N}$. $69^{\circ} 07^{-\mathrm{W}}$.

$\mathrm{GSCl}$ $40^{\circ} 52-$ $69^{\circ} 11^{-} \mathrm{W}$.

LYDONIA CANYON*

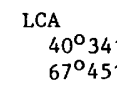

$\begin{array}{llrr}\text { LCB } & 145 & 92 & 190 \\ 40^{\circ} 32-\mathrm{N} . & 145 & 227 & 55 \\ 67^{\circ} 43^{-} \mathrm{W} . & 116 & 277\end{array}$

LCC $40^{\circ} 29-\mathrm{N}$ $116 \quad 134$ $67^{\circ} 44^{-} \mathrm{W}$.

LCD $40^{\circ} 29^{-} \mathrm{N}$. $67^{\circ} 41^{-}$W.

$\begin{array}{llll}\text { LCE } & 145 & 116 & 484\end{array}$

$40^{\circ} 25^{\circ} \mathrm{N}, \quad 145 \quad 216$ $67^{\circ} 40^{-}$W. $145 \quad 595$

LCF $145 \quad 205 \quad 300$ $174-405-100$
0 $67^{\circ} 39$-W.

LCG $\quad 174 \quad 195 \quad 300$
$40^{\circ} 21^{\prime}$ N. $174 \quad 395 \quad 100$ $67^{\circ} 42^{\circ} \mathrm{W}$.

$6.2+-1.5 \quad 131+-14$

$5.0+-0.9135+-10$

$2.0+-0.3 \quad 304+-10$

$5.0+-0.8 \quad 145+-7$

$8.6+-1.5109+-9$

$8.8+-1.0 \quad 112+-6$

$3.9+-0.7322+-9$

$8.2+-0.9118+-7$

$3.1+-1.3 \quad 119+-7$

$5.7+-0.7 \quad 127+-7$

$5.6+-0.7$ 125+- 7

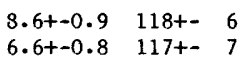

10.3
8.6

10.4
10.0

10.0
4.3

$9.4+-1.0$

$-1.9$

$-1.7$

116

117

$-1.9+-0.2$

$125+$

$4 \cdot 9+-1 \cdot 6$

$150+-$

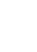


Table 8. $K_{1}$ tidal current parameters-Continued

Table 8. K1 TIDAL CURRENT PARAMETERS--Continued

\begin{tabular}{|c|c|c|c|}
\hline STATION & RECORD & INSTR & ABOVE \\
\hline $\begin{array}{l}\text { LAT. } \\
\text { LONG. }\end{array}$ & $\begin{array}{l}\text { LENGTH } \\
\text { (DAYS) }\end{array}$ & $\begin{array}{c}\text { DEPTH } \\
\text { (M) }\end{array}$ & $\begin{array}{l}\text { ВОTTOM } \\
\text { (M) }\end{array}$ \\
\hline
\end{tabular}

IA CANYON--CONT.

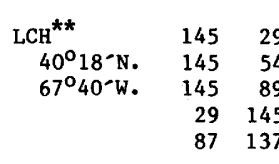

LCI $\quad 145 \quad 10 \quad 240$

$40^{\circ} 23-\mathrm{N} . \quad 145 \quad 55 \quad 195$

$67^{\circ} 33^{-W} \begin{array}{rrr}145 & 195 & 55 \\ 145 & 245\end{array}$

$\begin{array}{lrrr}\text { LCJ } & 116 & 83 & 488 \\ 40^{\circ} 21-\mathrm{N} . & 145 & 223 & 348\end{array}$

$\begin{array}{llll}40^{\circ} 21^{-} \mathrm{N} . & 145 & 223 & 348 \\ 67^{\circ} 32^{-} \mathrm{W} . & 145 & 471 & 100\end{array}$

LCK $\quad 145 \quad 204 \quad 350$

$40^{\circ} 16^{\circ}$ N. $145 \quad 454 \quad 100$

$67^{\circ} 47^{-W}$.

$\begin{array}{lrr}\text { LCL } & 116 & 65 \\ 40^{\circ} 32-\mathrm{N} . & 116 & 105\end{array}$

$67^{\circ} 36^{-W}$.

$\begin{array}{ccc}\text { LCM }^{*} & 145 & 103 \\ 40^{\circ} 30^{-} \text {N. } & 174 & 119\end{array}$

$40^{\circ} 30^{-} \mathrm{N}$.
$67^{\circ} 49^{\circ} \mathrm{W}$.

LCN $\quad 145 \quad 243 \quad 798$

$40^{\circ} 21-\mathrm{N} . \quad \begin{array}{lll}145 & 841 & 200\end{array}$ $67^{\circ} 40^{-} \mathrm{W}$.

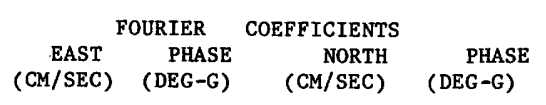

(DEG-G)

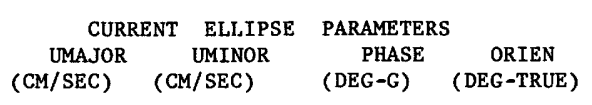

NANTUCKET SHOALS

\begin{tabular}{|c|c|c|c|c|c|c|c|c|c|c|c|c|c|}
\hline $\begin{array}{l}\text { NANTUCKET LS } \\
40^{\circ} 37^{\circ} \mathrm{N} . \\
69^{\circ} 37^{-} \mathrm{W}\end{array}$ & 738 & 2 & 53 & $8.2+-0.4$ & $159+-$ & 3 & $5.9+-0.1$ & $66+-$ & 5 & $8.2+-0.4$ & $-5 \cdot 8+-0.0$ & $162+-10$ & $94+-11$ \\
\hline $\begin{array}{l}\text { NSA } \\
\qquad \begin{array}{l}{ }^{\circ} \\
61^{-} \mathrm{N}\end{array} \\
69^{\circ} 36^{-} \mathrm{W} .\end{array}$ & $\begin{array}{l}60 \\
63\end{array}$ & $\begin{array}{r}5 \\
25\end{array}$ & $\begin{array}{r}28 \\
8\end{array}$ & $\begin{array}{l}0.7+-0.3 \\
1.4+-0.5\end{array}$ & $\begin{array}{l}60+- \\
63+-\end{array}$ & $\begin{array}{l}26 \\
20\end{array}$ & $\begin{array}{l}4 \cdot 4+-1 \cdot 3 \\
4 \cdot 5+-1.2\end{array}$ & $\begin{array}{l}357+- \\
357+-\end{array}$ & $\begin{array}{l}16 \\
16\end{array}$ & $\begin{array}{l}4 \cdot 4 \\
4 \cdot 5\end{array}$ & $\begin{array}{l}-0.6 \\
-1.3\end{array}$ & $\begin{array}{l}358 \\
359\end{array}$ & $\begin{array}{l}4 \\
8\end{array}$ \\
\hline $\begin{array}{l}\text { NSB } \\
\qquad \begin{array}{l}{ }^{\circ} 1_{26} \\
69^{\circ} \mathrm{N}\end{array} \\
69^{\circ}-\mathrm{W} .\end{array}$ & 42 & 10 & 12 & $3.4+-0.5$ & $40+-$ & 9 & $2.9+-0.4$ & $329+-$ & 1 & 3.7 & -2.5 & 17 & 58 \\
\hline $\begin{array}{l}\text { NSD } \\
\qquad 41^{\circ} 37^{\prime} \mathrm{N} . \\
69^{\circ} 44^{-} \mathrm{W} .\end{array}$ & 42 & 16 & 17 & $2 \cdot 3+-1 \cdot 2$ & $25+-$ & 30 & $3 \cdot 2+-1 \cdot 3$ & $306+-$ & 24 & 3.3 & $-2 \cdot 2$ & 316 & 15 \\
\hline
\end{tabular}

-.........

Instrument type, method of analysis and source of data are listed alphabetically by stations in Table 3. If UMINOR < 0 , ellipse rotates clockwise

* - tripod and subsurface mooring were at different depths.

** - Two separate moorings at different depths. 
Table 8. $K_{1}$ tidal current parameters-Continued

Table 8. K1 TIDAL CURRENT PARAMETERS--Continued

\begin{tabular}{|c|c|c|c|}
\hline STATION & RECORD & INSTR & ABOVE \\
\hline $\begin{array}{l}\text { LAT. } \\
\text { LONG. }\end{array}$ & $\begin{array}{l}\text { LENGTH } \\
\text { (DAYS) }\end{array}$ & $\begin{array}{l}\text { DEPTH } \\
\text { (M) }\end{array}$ & $\begin{array}{l}\text { BOTTOM } \\
\text { (M) }\end{array}$ \\
\hline
\end{tabular}

NANTUCKET SHOALS --CONT.

\begin{tabular}{|c|c|c|c|c|c|c|c|c|c|c|c|}
\hline $\begin{array}{c}\text { POLLOCK RIP } \\
41^{\circ} 37^{-} \mathrm{N} . \\
69^{\circ} 54^{-} \mathrm{W} .\end{array}$ & 369 & 2 & 12 & 0.1 & 267 & 1.6 & 228 & 1.6 & -0.1 & 228 & 3 \\
\hline $\begin{array}{c}\text { GREAT ROUND } \\
41^{\circ} 24^{-} \mathrm{N} \\
69^{\circ} 55^{-} \mathrm{W}\end{array}$ & 87 & 2 . & 20 & 5.2 & 51 & 0.2 & 128 & 5.2 & +0.1 & 51 & 90 \\
\hline $\begin{array}{l}\text { NSC } \\
\qquad 1^{\circ} 37^{-N} \text {. } \\
69^{\circ} 59^{-} \mathrm{W} .\end{array}$ & 42 & 8 & 8 & $4.5+-0.9$ & $44+-12$ & $1.3+-0.5$ & $251+-21$ & 4.6 & -0.6 & 226 & 285 \\
\hline $\begin{array}{l}\text { I } \\
40^{\circ} 43^{-} \mathrm{N} \\
70^{\circ} 01^{-} \mathrm{W}\end{array}$ & 29 & 18 & 23 & 9.0 & 153 & 4.9 & 24 & 9.6 & -3.5 & 342 & 293 \\
\hline $\begin{array}{l}\text { NSE } \\
\qquad 0^{\circ} 59^{\circ} \mathrm{N} . \\
70^{\circ} 04^{-} \mathrm{W} .\end{array}$ & 41 & 10 & 12 & $5.4+-2.2$ & $67+-23$ & $7.2+-3.7$ & $313+-30$ & 7.7 & -4.6 & 296 & 333 \\
\hline $\begin{array}{l}\text { NSFE1 } \\
\quad 40^{\circ} 41-\mathrm{N} . \\
70^{\circ} 08^{-} \mathrm{W} .\end{array}$ & $\begin{array}{l}172 \\
222\end{array}$ & $\begin{array}{l}10 \\
30\end{array}$ & $\begin{array}{l}36 \\
16\end{array}$ & $\begin{array}{r}11.0+-1.0 \\
7.4+-0.9\end{array}$ & $\begin{array}{ll}159+- & 5 \\
169+- & 6\end{array}$ & $\begin{array}{r}10.6+-1.0 \\
5.7+-0.7\end{array}$ & $\begin{array}{ll}45+- & 5 \\
55+- & 6\end{array}$ & $\begin{array}{r}12.8 \\
8.0\end{array}$ & $\begin{array}{l}-8.4 \\
-4.8\end{array}$ & $\begin{array}{r}10 \\
7\end{array}$ & $\begin{array}{l}313 \\
299\end{array}$ \\
\hline $\begin{array}{l}Q \\
40^{\circ} 30^{-} \mathrm{N} . \\
70^{\circ} 13^{-} \mathrm{W}\end{array}$ & $\begin{array}{l}174 \\
464 \\
377 \\
145 \\
116\end{array}$ & $\begin{array}{l}10 \\
31 \\
51 \\
57 \\
66\end{array}$ & $\begin{array}{r}57 \\
36 \\
16 \\
10 \\
1\end{array}$ & $\begin{array}{l}8.7+-0.8 \\
7.3+-1.1 \\
6.3+-0.9 \\
5.4+-1.0 \\
3.9+-1.1\end{array}$ & $\begin{array}{ll}169+- & 5 \\
173+- & 8 \\
188+- & 9 \\
199+- & 6 \\
185+- & 8\end{array}$ & $\begin{array}{l}7.4+-0.9 \\
5.7+-1.1 \\
4.8+-0.7 \\
3.5+-0.6 \\
2.6+-0.9\end{array}$ & $\begin{array}{r}68+-6 \\
70+-12 \\
91+-16 \\
105+-11 \\
78+-11\end{array}$ & $\begin{array}{l}9.0+-0.7 \\
7.6+-1.1 \\
6.4+-0.8 \\
5.4+-1.0 \\
4.0+-1.2\end{array}$ & $\begin{array}{l}-7.0+-1.0 \\
-5.3+-1.2 \\
-4.6+-0.8 \\
-3.5+-0.5 \\
-2.4+-0.7\end{array}$ & $\begin{array}{rr}8+- & 8 \\
7+- & 10 \\
12+- & 13 \\
22+- & 4 \\
15+- & 6\end{array}$ & $\begin{array}{lr}294+- & 8 \\
290+- & 9 \\
277+- & 16 \\
281+- & 11 \\
287+- & 7\end{array}$ \\
\hline $\begin{array}{l}\text { NSFE3 } \\
\quad 40^{\circ} 20^{-} \mathrm{N} \\
70^{\circ} 16^{-} \mathrm{W}\end{array}$ & $\begin{array}{r}46 \\
209 \\
209\end{array}$ & $\begin{array}{l}10 \\
30 \\
70\end{array}$ & $\begin{array}{l}78 \\
58 \\
18\end{array}$ & $\begin{array}{l}6.2+-4.1 \\
5.2+-0.5 \\
5.2+-0.9\end{array}$ & $\begin{array}{lr}188+- & 38 \\
178+- & 8 \\
186+- & 10\end{array}$ & $\begin{array}{l}4.4+-3.0 \\
3.7+-0.7 \\
4.2+-0.9\end{array}$ & $\begin{array}{l}97+-40 \\
82+-11 \\
87+-12\end{array}$ & $\begin{array}{l}6.2 \\
5.2 \\
5.3\end{array}$ & $\begin{array}{l}-4 \cdot 4 \\
-3 \cdot 7 \\
-4 \cdot 1\end{array}$ & $\begin{array}{r}9 \\
4 \\
20\end{array}$ & $\begin{array}{l}271 \\
278 \\
288\end{array}$ \\
\hline $\begin{array}{l}\text { NSFE4 } \\
\quad 40^{\circ} 13^{\circ} \mathrm{N} . \\
70^{\circ} 18^{-} \mathrm{W} .\end{array}$ & $\begin{array}{l}170 \\
224 \\
170 \\
224\end{array}$ & $\begin{array}{l}10 \\
30 \\
60 \\
90\end{array}$ & $\begin{array}{l}95 \\
75 \\
45 \\
15\end{array}$ & $\begin{array}{l}6.2+-1.1 \\
4.2+-0.7 \\
5.7+-0.8 \\
5.0+-0.9\end{array}$ & $\begin{array}{lr}178+- & 10 \\
181+- & 9 \\
182+- & 8 \\
186+- & 10\end{array}$ & $\begin{array}{l}5.2+-0.9 \\
3.1+-0.9 \\
4.5+-0.7 \\
4.0+-0.7\end{array}$ & $\begin{array}{rr}87+- & 10 \\
92+- & 16 \\
78+- & 9 \\
100+- & 10\end{array}$ & $\begin{array}{l}6.2 \\
4.2 \\
5.9 \\
5.0\end{array}$ & $\begin{array}{l}-5.2 \\
-3.1 \\
-4.3 \\
-4.0\end{array}$ & $\begin{array}{r}1 \\
0 \\
19 \\
359\end{array}$ & $\begin{array}{l}273 \\
268 \\
293 \\
261\end{array}$ \\
\hline $\begin{array}{l}\text { NSFE } 5^{*} \\
\quad 40^{\circ} 02^{-} \mathrm{N} \\
70^{\circ} 22^{-} \mathrm{W}\end{array}$ & $\begin{array}{l}171 \\
171 \\
171 \\
171 \\
171\end{array}$ & $\begin{array}{r}10 \\
30 \\
90 \\
120 \\
185\end{array}$ & $\begin{array}{r}188 \\
168 \\
108 \\
78 \\
13\end{array}$ & $\begin{array}{l}4.4+-1.3 \\
3.5 \\
3.1+-0.6 \\
2.8+-0.5 \\
3.2+-0.6\end{array}$ & $\begin{array}{l}187+-15 \\
172 \\
188+-10 \\
183+-11 \\
190+-10\end{array}$ & $\begin{array}{l}4.0+-1.5 \\
2.5 \\
2.7+-0.5 \\
2.7+-0.5 \\
3.0+-0.5\end{array}$ & $\begin{array}{l}95+-22 \\
73 \\
93+-10 \\
81+-11 \\
91+-10\end{array}$ & $\begin{array}{l}4.4 \\
3.5 \\
3.1 \\
3.0 \\
3.4\end{array}$ & $\begin{array}{l}-4.0 \\
-2.4 \\
-2.7 \\
-2.5 \\
-2.8\end{array}$ & $\begin{array}{r}20 \\
1 \\
23 \\
40 \\
41\end{array}$ & $\begin{array}{l}284 \\
283 \\
288 \\
313 \\
306\end{array}$ \\
\hline $\begin{array}{l}\text { NSFE6 } \\
\quad 39^{\circ} 51^{-N} \text {. } \\
70^{\circ} 25^{-} \text {W. }\end{array}$ & 225 & 10 & 800 & $0.9+-0.4$ & $243+-26$ & $1.1+-0.7$ & $149+-35$ & 1.1 & -0.9 & 141 & 351 \\
\hline
\end{tabular}

$\begin{array}{crrr}\text { FOURIER } & \text { COEFFICIENTS } & \\ \text { EAST } & \text { PHASE } & \text { NORTH } & \text { PHASE } \\ (\mathrm{CM} / \mathrm{SEC}) & (\mathrm{DEG}-\mathrm{G}) & (\mathrm{CM} / \mathrm{SEC}) & (\mathrm{DEG}-\mathrm{G})\end{array}$
CURRENT ELLIPSE PARAMETERS IMAJOR UMINOR (CM/SEC) (DEG-G) (DEG-TRUE)

Instrument type, method of analysis and source of data are listed alphabetically by stations in Table 3 . If UMINOR < 0 , ellipse rotates clockwise

* Orientation is questionable 
Table 8. $K_{1}$ tidal current parameters-Continued

Table 8. K1 TIDAL CURRENT PARAMETERS--Cont1nued

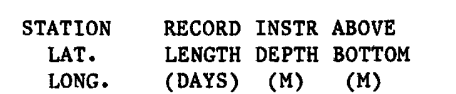

LONG. (DAYS) (M) (M)

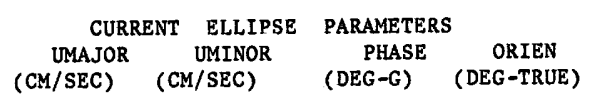

NEW ENGLAND SHELF

P $\begin{array}{lll}40^{\circ} 29^{-} \mathrm{N} . & 87 & 6 \\ 70^{\circ} 30^{-} \mathrm{W} . & 58 & 70\end{array}$

NES7 43 $40^{\circ} 18-\mathrm{N} . \quad 35 \quad 20$ $70^{\circ} 52^{\circ} \mathrm{W}$.

NES764 $\begin{array}{rrrr}39^{\circ} 37^{\circ} \mathrm{N} . & 180 & 305 & 1995 \\ 780 & 2005 & 295\end{array}$ $70^{\circ} 56^{\circ} \mathrm{W}$.

NES7 42 $40^{\circ} 35-\mathrm{N}$. $70^{\circ} 59^{\circ} \mathrm{W}$.

HENS\&CHICK 369 $41^{\circ} 27^{-N}$. $71^{\circ} \mathrm{O1}-\mathrm{W}$.

NES763 $39^{\circ} 56^{\circ} \mathrm{N}$. $71^{\circ} 03-\mathrm{W}$.

NES 762 \begin{tabular}{l}
$40^{\circ} 28^{-\mathrm{N}}$ \\
\hline
\end{tabular} $71^{\circ} 12^{-} \mathrm{W}$.

NES741 $40^{\circ} 56-\mathrm{N}$. $71^{\circ} 13^{-} \mathrm{W}$.

BRENTON REFF 369 $41^{\circ} 26^{-} \mathrm{N}$. $71^{\circ} 23-w$.

MIDDLE ATLANTIC BIGHT

$\begin{array}{crrr} & \text { FOURIER } & \text { COEFFICIENTS } & \\ \text { EAST } & \text { PHASE } & \text { NORTH } & \text { PHASE } \\ (\mathrm{CM} / \mathrm{SEC}) & (\mathrm{DEG}-\mathrm{G}) & (\mathrm{CM} / \mathrm{SEC}) & (\mathrm{DEG}-\mathrm{G})\end{array}$

$\begin{array}{cccc}\text { UMAJOR } & \text { UMINOR } & \text { PHASE } & \text { ORIEN } \\ (\mathrm{CM} / \mathrm{SEC}) & (\mathrm{CM} / \mathrm{SEC}) & \text { (DEG-G) } & \text { (DEG-TRUE) }\end{array}$

$4.1+-0.4 \quad-3.2+-0.6 \quad 181+-16 \quad 80+-10$ $2.2+-0.1 \quad-1.2+-0.1 \quad 177+-20 \quad 79+-6$

$4.0+-0.5 \quad 189+-15 \quad 3.2+-0.6 \quad 104+-13$ $2.2+-0.0 \quad 183+-23 \quad 1.3+-0.1 \quad 106+-28$

$2.9+-0.6 \quad 197+-12 \quad 2.0+-1.2 \quad 92+-34$ $\begin{array}{lllr}7.1+-3.1 & 216+-25 & 6.6+-2.8 & 126+-25\end{array}$

3.0

$-1.9$

-1.9
-6.6

28

36

287

$0.2+-0.2 \quad 255+-56, \quad 0.5+-0.3 \quad 118+-31$ $0.4+-0.1 \quad 20+-960.2+-0.1 \quad 101+-37$

0.5

0.4

$-0.1$

$+0.2$

114

203

343

$4.8+-1.2 \quad 196+-14 \quad 2.7+-1.2 \quad 95+-26$ $6.9+-2.3 \quad 217+-19 \quad 5.1+-2.6 \quad 128+-29$

4.8
6.9

$-2.6$

1.7

$0.3 \quad 68$

1.796

$96 \quad 0.3$

$181 \quad 145 \quad 351$

$1.4+-0.3 \quad 222+-1$

$1.2+-0.3 \quad 150+-13$

1.5

$-1 \cdot 1$

199

58

\section{$13938 \quad 45 \quad 3.7+-0.5 \quad 216+-8 \quad 2.6+-0.6 \quad 131+-12$}

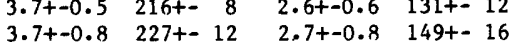

3.7

$-2.6$

4.7

$4.7+-1.1 \quad 202+-14 \quad 2.2+-0.7 \quad 101+-18$

2.0 NES $763 \mathrm{~W}$
$39^{\circ} 43^{-} \mathrm{N}$ $183 \quad 302 \quad 202$

$71^{\circ} 47^{-} \mathrm{W}$.
0.7

183

2.0 
Table 8. $K_{1}$ tidal current parameters-Continued

Table 8. K1 TLDAL CURRENT PARAMETERS--Continued

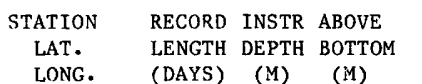

MIDDLE ATLANTIC BIGHT--CONT.

\begin{tabular}{|c|c|c|c|c|c|c|c|c|c|c|c|}
\hline $\begin{array}{l}\text { NES762W } \\
39^{\circ} 55^{-} \mathrm{N} . \\
71^{\circ} 58^{-} \mathrm{W} .\end{array}$ & 172 & 38 & 45 & $3.1+-0.5$ & $267+-10$ & $2.3+-0.5$ & $191+-13$ & 3.2 & -2.2 & 254 & 71 \\
\hline $\begin{array}{l}\text { LT5 } \\
\qquad 0^{\circ} 12^{-} \mathrm{N} \\
72^{\circ} 00^{-} \mathrm{W}\end{array}$ & $\begin{array}{r}87 \\
70 \\
116 \\
70 \\
87 \\
70 \\
87 \\
70\end{array}$ & $\begin{array}{l}21-\mathrm{H} \\
21-\mathrm{R} \\
41-\mathrm{H} \\
41-\mathrm{R} \\
61-\mathrm{H} \\
61-\mathrm{R} \\
66-\mathrm{H} \\
66-\mathrm{R}\end{array}$ & $\begin{array}{r}46 \\
46 \\
26 \\
26 \\
6 \\
6 \\
1 \\
1\end{array}$ & $\begin{array}{l}5.8+-0.9 \\
4.6+-1.5 \\
3.9+-2.0 \\
4.7+-1.0 \\
2.1+-0.3 \\
2.3+-0.4 \\
1.3+-0.3 \\
1.6+-0.3\end{array}$ & $\begin{array}{l}232+-18 \\
243+-29 \\
255+-14 \\
254+-12 \\
286+-9 \\
284+-10 \\
273+-38 \\
295+-11\end{array}$ & $\begin{array}{l}4.2+-1.5 \\
3.2+-1.2 \\
2.8+-0.8 \\
2.7+-0.9 \\
2.2+-0.1 \\
2.1+-0.3 \\
1.3+-0.6 \\
1.7+-0.3\end{array}$ & $\begin{array}{lr}161+- & 16 \\
190+- & 22 \\
189+- & 15 \\
178+- & 29 \\
229+- & 0 \\
228+- & 9 \\
232+- & 6 \\
237+- & 9\end{array}$ & $\begin{array}{l}6.4+-1.1 \\
5.1 \\
4.3+-1.7 \\
4.8 \\
2.7+-0.2 \\
2.8 \\
1.7+-0.5 \\
2.0\end{array}$ & $\begin{array}{l}-3.3+-1.3 \\
-2.3 \\
-2.2+-1.2 \\
-2.6 \\
-1.4+-0.1 \\
-1.5 \\
-0.7+-0.6 \\
-1.1\end{array}$ & $\begin{array}{l}238+-45 \\
229 \\
223+-39 \\
248 \\
254+-\quad 4 \\
260 \\
246+-17 \\
263\end{array}$ & $\begin{array}{l}90+-41 \\
61 \\
46+-40 \\
79 \\
42+-10 \\
50 \\
43+-16 \\
42\end{array}$ \\
\hline $\begin{array}{l}\text { LT4 } \\
\quad 40^{\circ} 34^{-} \mathrm{N} . \\
72^{\circ} 19^{-} \mathrm{W}\end{array}$ & $\begin{array}{r}29 \\
203 \\
174 \\
87\end{array}$ & $\begin{array}{r}3 \\
24 \\
44 \\
51\end{array}$ & $\begin{array}{r}49 \\
28 \\
8 \\
1\end{array}$ & $\begin{array}{l}5.8+-0.6 \\
5.9+-2.0 \\
3.4+-1.2 \\
1.9+-0.4\end{array}$ & $\begin{array}{l}191+-30 \\
245+-10 \\
253+-21 \\
271+-\quad 3\end{array}$ & $\begin{array}{l}3.3+-0.2 \\
2.3+-0.8 \\
2.0+-1.3 \\
1.4+-0.4\end{array}$ & $\begin{array}{r}81+-66 \\
214+-50 \\
225+-48 \\
242+-10\end{array}$ & $\begin{array}{l}6.1+-0.2 \\
6.1+-1.9 \\
3.8+-1.4 \\
2.3+-0.5\end{array}$ & $\begin{array}{l}-2.7+-0.7 \\
-1.0+-1.8 \\
-0.8+-1.5 \\
-0.6+-0.4\end{array}$ & $\begin{array}{r}195+-107 \\
246+-11 \\
250+-23 \\
261+-\quad 1\end{array}$ & $\begin{array}{rr}102+- & 23 \\
73+- & 10 \\
64+- & 11 \\
56+- & 0\end{array}$ \\
\hline $\begin{array}{l}\text { CMICE } \\
\quad 40^{\circ} 47^{-\mathrm{N}} \\
72^{\circ} 29^{-\mathrm{W}}\end{array}$ & $\begin{array}{l}25 \\
25 \\
25 \\
25\end{array}$ & $\begin{array}{r}4 \\
8 \\
16 \\
25\end{array}$ & $\begin{array}{r}25 \\
21 \\
13 \\
4\end{array}$ & $\begin{array}{l}3.4+-1.1 \\
3.8+-1.0 \\
2.6+-1.1 \\
0.5+-0.2\end{array}$ & $\begin{array}{l}248+-18 \\
249+-14 \\
223+-24 \\
208+-32\end{array}$ & $\begin{array}{l}1.5+-1.4 \\
1.2+-1.2 \\
0.7+-0.6 \\
1.7+-0.7\end{array}$ & $\begin{array}{r}172+-56 \\
151+-57 \\
37+-48 \\
332+-28\end{array}$ & $\begin{array}{l}3.4 \\
3.8 \\
2.7 \\
1.7\end{array}$ & $\begin{array}{l}-1.4 \\
-1.2 \\
+0.1 \\
+0.4\end{array}$ & $\begin{array}{l}245 \\
250 \\
223 \\
334\end{array}$ & $\begin{array}{r}83 \\
93 \\
105 \\
350\end{array}$ \\
\hline $\begin{array}{l}\mathrm{ME} \\
39^{\circ} 57^{-\mathrm{N}} . \\
72^{\circ} 36^{\prime} \mathrm{W} .\end{array}$ & 29 & 59 & 1 & 1.6 & 268 & 0.5 & 176 & 1.6 & -0.5 & 88 & 271 \\
\hline $\begin{array}{l}\mathrm{NJ} 4 \\
38^{\circ} 55^{-N} \text {. } \\
72^{\circ} 58^{\prime} \mathrm{W} .\end{array}$ & $\begin{array}{l}72 \\
72 \\
72\end{array}$ & $\begin{array}{r}3 \\
43 \\
91\end{array}$ & $\begin{array}{r}89 \\
49 \\
1\end{array}$ & $\begin{array}{l}2.2+-1.4 \\
0.9+-0.3 \\
0.2+-0.0\end{array}$ & $\begin{array}{r}208+-37 \\
7+-21 \\
67+-11\end{array}$ & $\begin{array}{l}2.5+-1.1 \\
0.7+-0.2 \\
0.4+-0.1\end{array}$ & $\begin{array}{r}118+-26 \\
331+-18 \\
50+-10\end{array}$ & $\begin{array}{l}2.5 \\
1.1 \\
0.4\end{array}$ & $\begin{array}{l}-2.2 \\
-0.3 \\
-0.1\end{array}$ & $\begin{array}{r}118 \\
354 \\
53\end{array}$ & $\begin{array}{r}0 \\
54 \\
26\end{array}$ \\
\hline $\begin{array}{l}\text { MA } \\
\begin{array}{l}39^{\circ} 27^{-} \mathrm{N} \\
73^{\circ} 00^{-} \mathrm{W}\end{array}\end{array}$ & 58 & 58 & 1 & $0.5+-0.1$ & $209+-120$ & $0.6+-0.2$ & $356+-60$ & $0.7+-0.0$ & $-0.1+-0.4$ & $351+-69$ & $26 t-33$ \\
\hline $\begin{array}{l}\text { LT3 } \\
39^{\circ} 16^{-} \mathrm{N} \\
73^{\circ} 02^{-} \mathrm{W} .\end{array}$ & $\begin{array}{r}104 \\
104 \\
70\end{array}$ & $\begin{array}{r}9 \\
19 \\
58\end{array}$ & $\begin{array}{l}61 \\
51 \\
12\end{array}$ & $\begin{array}{l}1.5+-0.8 \\
1.3+-0.8 \\
0.7+-0.3\end{array}$ & $\begin{array}{l}90+-30 \\
77+-34 \\
60+-23\end{array}$ & $\begin{array}{l}1.5+-0.5 \\
2.2+-0.7 \\
1.2+-0.2\end{array}$ & $\begin{array}{r}24+-18 \\
7+-18 \\
354+-10\end{array}$ & $\begin{array}{l}1.8 \\
2.3 \\
1.2\end{array}$ & $\begin{array}{l}-1.2 \\
-1.2 \\
-0.6\end{array}$ & $\begin{array}{r}57 \\
16 \\
3\end{array}$ & $\begin{array}{l}45 \\
16 \\
18\end{array}$ \\
\hline $\begin{array}{l}\text { MESA7 } \\
\quad 39^{\circ} 55^{-} \mathrm{N} \\
73^{\circ} 06^{-} \mathrm{W} .\end{array}$ & $\begin{array}{r}107 \\
103 \\
63\end{array}$ & $\begin{array}{l}18 \\
38 \\
66\end{array}$ & $\begin{array}{r}50 a \\
30 a \\
2 a\end{array}$ & $\begin{array}{l}0.8+-0.6 \\
0.8+-0.4 \\
0.4+-0.2\end{array}$ & $\begin{array}{l}244+-45 \\
277+-30 \\
253+-30\end{array}$ & $\begin{array}{l}1.0+-0.3 \\
1.7+-0.8 \\
0.4+-0.1\end{array}$ & $\begin{array}{r}8+-18 \\
246+-28 \\
352+-10\end{array}$ & $\begin{array}{l}1.1 \\
1.8 \\
0.4\end{array}$ & $\begin{array}{l}+0.6 \\
-0.4 \\
+0.4\end{array}$ & $\begin{array}{r}27 \\
251 \\
33\end{array}$ & $\begin{array}{r}326 \\
23 \\
315\end{array}$ \\
\hline FIRE IS. & 369 & 2 & 27 & 2.2 & 244 & 0.8 & 171 & 2.2 & -0.8 & 242 & 83 \\
\hline
\end{tabular}

$\begin{array}{crrr} & \text { FOURIER } & \text { COEFFICIENTS } & \\ \text { EAST } & \text { PHASE } & \text { NORTH } & \text { PHASE } \\ (\mathrm{CM} / \mathrm{SEC}) & (\mathrm{DEG}-\mathrm{G}) & (\mathrm{CM} / \mathrm{SEC}) & (\mathrm{DEG}-\mathrm{G})\end{array}$

(DEG-G)

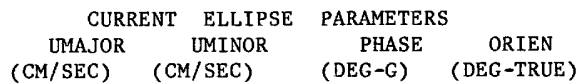

$73^{\circ} 11^{-W}$.

Instrument type, method of analysis and source of data are listed alphabetically by stations in Table 3 . If: UMINOR $<0$, ellipse rotates clockwise

$H$ - harmonic analysis $R$ - response analysis

a - water depth taken from chart 13200 
Table 8. $K_{1}$ tidal current parameters-Continued

Table 8. K1 TIDAL CURRENT PARAMETERS--Continued

\begin{tabular}{cllllrrr} 
STATION & RECORD INSTR ABOVE & \multicolumn{3}{c}{ FOURIER } & COEFFICIENTS \\
LAT. & LENGTH & DEPTH BOTTOM & EAST & PHASE & NORTH & PHASE \\
LONG. & (DAYS) & (M) & (M) & $(\mathrm{CM} / \mathrm{SEC})$ & $(\mathrm{DEG}-\mathrm{G})$ & $(\mathrm{CM} / \mathrm{SEC})$ & (DEG-G)
\end{tabular}

\begin{tabular}{cccc}
\multicolumn{2}{c}{ CURRENT } & ELLIPSE & PARAMETERS \\
UMAJOR & UMINOR & PHASE & ORIEN \\
$(\mathrm{CM} / \mathrm{SEC})$ & $(\mathrm{CM} / \mathrm{SEC})$ & (DEG-G) & (DEG-TRUE)
\end{tabular}

MIDDLE ATLANTIC BIGHT--CONT.

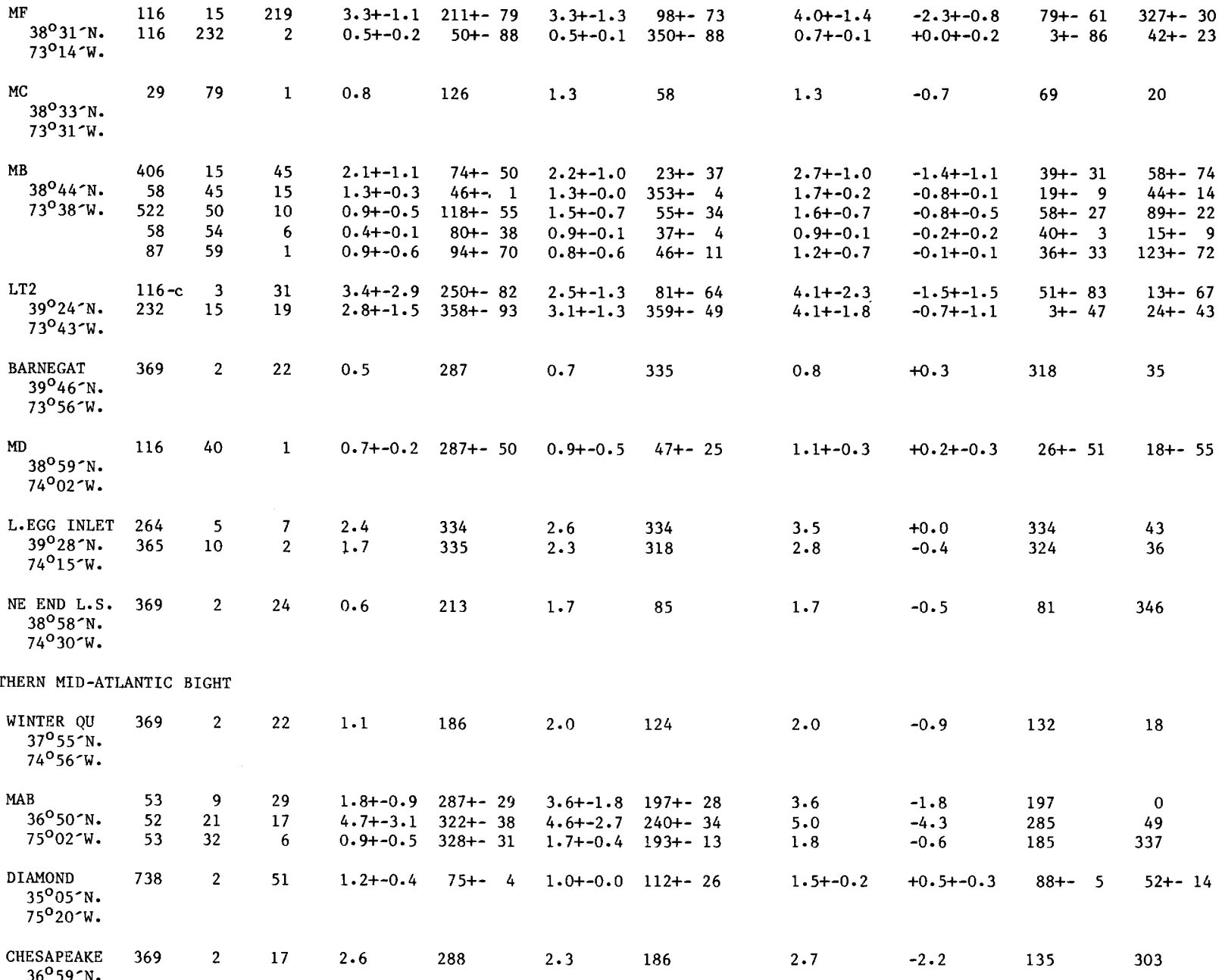

Instrument type, method of analysis and source of data are listed alphabetically by stations in Table 3. If UMINOR < 0, ellipse rotates clockwise

c - average of 4 records $31,31,32$, and 29 meters above bottom 
Table 8. $K_{1}$ tidal current parameters-Continued

Table 8. K1 TIDAL CURRENT PARAMETERS--Continued

\begin{tabular}{|c|c|c|c|c|c|c|c|c|c|c|c|}
\hline STATION & RECORD & INSTR & ABOVE & & OURIER & COEFFICIENTS & & CURF & ELLIPSE & PARAMETERS & \\
\hline $\begin{array}{l}\text { LAT. } \\
\text { LONG. }\end{array}$ & $\begin{array}{l}\text { LENGTH } \\
\text { (DAYS) }\end{array}$ & $\begin{array}{l}\text { DEPTH } \\
\text { (M) }\end{array}$ & $\begin{array}{l}\text { BOTTOM } \\
\text { (M) }\end{array}$ & $\begin{array}{c}\text { EAST } \\
(\mathrm{CM} / \mathrm{SEC})\end{array}$ & $\begin{array}{r}\text { PHASE } \\
\text { (DEG-G) }\end{array}$ & $\begin{array}{r}\text { NORTH } \\
(\mathrm{CM} / \mathrm{SEC})\end{array}$ & $\begin{array}{l}\text { PHASE } \\
(\mathrm{DEG}-\mathrm{G})\end{array}$ & $\begin{array}{l}\text { UMAJOR } \\
\text { (CM/SEC) }\end{array}$ & $\begin{array}{l}\text { UMINOR } \\
\text { (CM/SEC) }\end{array}$ & $\begin{array}{c}\text { PHASE } \\
(D E G-G)\end{array}$ & $\begin{array}{c}\text { ORIEN } \\
\text { (DEG-TRUE) }\end{array}$ \\
\hline
\end{tabular}

OCEANIC

\begin{tabular}{|c|c|c|c|c|c|c|c|c|c|c|c|}
\hline $\begin{array}{l}\mathrm{S} 8 \\
42^{\circ} 00^{-} \mathrm{N} \\
63^{\circ} 30^{-} \mathrm{W}\end{array}$ & $\begin{array}{r}415 \\
415 \\
96\end{array}$ & $\begin{array}{r}70 \\
1500 \\
2530\end{array}$ & $\begin{array}{r}2470 \\
1040 \\
10\end{array}$ & $\begin{array}{l}0.5+-0.1 \\
0.1+-0.1 \\
0.2\end{array}$ & $\begin{array}{rr}98+- & 44 \\
52+- & 2 \\
144 & \end{array}$ & $\begin{array}{l}0.2+-0.1 \\
0.1+-0.1 \\
0.3\end{array}$ & $\begin{array}{l}318+-27 \\
219+-16 \\
246\end{array}$ & $\begin{array}{l}0.6+-0.1 \\
0.2+-0.1 \\
0.3\end{array}$ & $\begin{array}{l}-0.1+-0.1 \\
+0.0+-0.0 \\
+0.2\end{array}$ & $\begin{array}{lr}282+- & 39 \\
225+- & 9 \\
255 & \end{array}$ & $\begin{array}{l}285+- \\
315+- \\
347\end{array}$ \\
\hline $\begin{array}{l}\text { NES765 } \\
39^{\circ} 17^{-\mathrm{N}} . \\
70^{\circ} 50^{-} \mathrm{W}\end{array}$ & 180 & 1995 & 655 & $0.2+-0.0$ & $8+-12$ & $0.1+-0.0$ & $110+-16$ & 0.2 & +0.1 & 184 & 278 \\
\hline
\end{tabular}

Instrument type, method of analysis and source of data are listed alphabetically by stations in Table 3 .

If $U M I N O R<0$, ellipse rotates clockwise 
Table 9. $\mathrm{O}_{1}$ tidal current parameters (see table 5 for explanation).

Table 9. 01 TIDAL CURRENT PARAMETERS

$\begin{array}{cl}\text { STATION } & \text { RECORD INSTR ABOVE } \\ \text { LAT. } & \text { LENGTH DEPTH BOTTOM }\end{array}$

LONG. (DAYS) (M) (M)

$\begin{array}{cccc} & \text { FOURIER } & \text { COEFFICIENTS } & \\ \text { EAST } & \text { PHASE } & \text { NORTH } & \text { PHASE } \\ (\mathrm{CM} / \mathrm{SEC}) & (\mathrm{DEG}-\mathrm{G}) & (\mathrm{CM} / \mathrm{SEC}) & (\mathrm{DEG}-\mathrm{G})\end{array}$

SCOTIAN SHELF

SS10

$32 \quad 200 \quad 1400$

$59^{\circ} 04^{-W}$.

SS2A

$42^{\circ} 52^{-\mathrm{N}}$

$62^{\circ} 00^{-} \mathrm{W}$.

SS8

$42^{\circ} 37^{-} \mathrm{N}$.

$62^{\circ} 05^{-\mathrm{W}}$.

SS1A

$42^{\circ} 52^{\circ}$

$62^{\circ} 14-\mathrm{W}$.

SS3

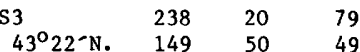

$62^{\circ} 40^{\circ} \mathrm{W}$

$15 \quad 91$

5

$\begin{array}{llr}43^{\circ} 02-\mathrm{N} . & 27 & 50 \\ 62^{\circ} 54-W . & 118\end{array}$

$62^{\circ} 54-\mathrm{W}$.

$\begin{array}{lrrr}\text { SS2 } & 189 & 20 & 258 \\ 43^{\circ} 45^{-} \mathrm{N} . & 283 & 50 & 228 \\ 62^{\circ} 59^{-} \mathrm{W} . & 166 & 95 & 18 \\ & 272 & 250 & 28\end{array}$

$\begin{array}{llr}\text { SS6 } & 26 & 50 \\ 43^{\circ} 15^{-N} . & 27 & 130\end{array}$

$63^{\circ} 22^{-} \mathrm{W}$.

$\begin{array}{lll}\mathrm{SS} 1 & 211 & 14 \\ 44^{\circ} 26^{-} \mathrm{N} & 187 & 95\end{array}$ $63^{\circ} 29^{\circ} \mathrm{W}$.

S5 $\begin{array}{rrrr}262 & 50 & 1470 \\ 42^{\circ} 30-\mathrm{N} . & 262 & 150 & 1370\end{array}$

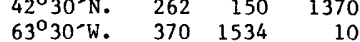

$\begin{array}{ll}0.3 & 311 \\ 0.2 & 282\end{array}$

$0.2 \quad 318$

0.2

108

0.1

113

0.2

0.3

144

$5.8+-0.7 \quad 216+-30 \quad 3.8+-0.5 \quad 153+-13$

$5.9+-1.3 \quad 215+-\quad 5 \quad 4.3+-0.7 \quad 142+-1$

4.5

237

5.5

279

3.4

3.7

177

$6 \cdot 8$

221

4.4

221

$3.5 \quad 256$

3.6

154

$4.3+-0.9223+-40$

$2.5+-0.5 \quad 261+-14 \quad 3.4+-0.6 \quad 280+-5$

3.9251

1.7

202

249

2.4

250

$5.8+-0.8 \quad 220+-88 \quad 2.3+-0.3 \quad 182+-76$

$\begin{array}{rrrr}1.2+-0.6 & 21+-92 & 2.7+-1.2 & 269+-70\end{array}$

$0.6+-0.0 \quad 238+-6 \quad 0.7+-0.3 \quad 166+-11$

$0.4+-0.0 \quad 283+-38$

$\begin{array}{ll}0.4+-0.0 & 283+- \\ 0.3 & 26\end{array}$

$\begin{array}{ll}0.7+-0.3 & 166+-11 \\ 0.3+-0.1 & 154+-20\end{array}$

$\begin{array}{ll}0.3+-0.1 & 154+- \\ 0.2 & 172\end{array}$ $\begin{array}{ll}0.6 & 218 \\ 0.4 & 255\end{array}$

3. $2+-1.1245+-12$

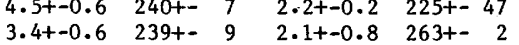

\begin{tabular}{cc}
\multicolumn{3}{c}{ CURRENT } & ELLIPSE \\
UMAJOR & UMINOR \\
(CM/SEC) & $(\mathrm{CM} / \mathrm{SEC})$
\end{tabular}

PARAMETERS

PHASE ORIEN

(DEG-G) (DEG-TRUE)

- - - -

Instrument type, method of analysis and source of data are 1isted alphabetically by stations in Table 3 .

If UMINOR $<0$, ellipse rotates clockwise 
Table 9. $\mathrm{O}_{1}$ tidal current parameters-Continued

Table 9. 01 TIDAL CURRENT PARAMETERS--Continued

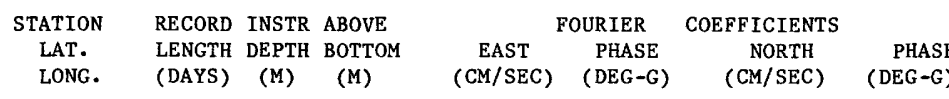

\begin{tabular}{llllllll} 
TATION & RECORD INSTR ABOVE & \multicolumn{3}{c}{ FOURIER } & COEFFICIENTS \\
LAT. & LENGTH DEPTH BOTTOM & EAST & PHASE & NORTH & PHASE \\
LONG. & (DAYS) & (M) & (M) & (CM/SEC) & (DEG-G) & $($ (CM/SEC) & $($ DEG-G)
\end{tabular}

SCOTIAN SHELF--CONT.

\begin{tabular}{|c|c|c|c|c|c|c|c|c|c|c|c|}
\hline $\begin{array}{l}\mathrm{SS} 5 \\
42^{\circ} 35^{\circ} \mathrm{N} \\
63^{\circ} 30^{-} \mathrm{W} .\end{array}$ & 14 & 150 & 1575 & 0.4 & 336 & 0.3 & 196 & 0.5 & -0.2 & 169 & 305 \\
\hline $\begin{array}{l}\text { SS4 } \\
\quad 42^{\circ} 40^{-} \mathrm{N} . \\
63^{\circ} 30^{-} \mathrm{W} .\end{array}$ & $\begin{array}{r}94 \\
585 \\
204 \\
663 \\
363 \\
210 \\
296\end{array}$ & $\begin{array}{r}20 \\
50 \\
100 \\
150 \\
500 \\
690 \\
990\end{array}$ & $\begin{array}{r}1000 \\
970 \\
920 \\
870 \\
520 \\
330 \\
30\end{array}$ & $\begin{array}{l}2.6+-0.4 \\
1.7+-0.1 \\
1.0 \\
1.3+-0.3 \\
0.5+-0.1 \\
0.3+-0.2 \\
0.7+-0.3\end{array}$ & $\begin{array}{rr}262+- & 6 \\
252+- & 31 \\
304 & \\
286+- & 9 \\
28+- & 98 \\
29+- & 16 \\
9+- & 38\end{array}$ & $\begin{array}{l}1.9+-0.5 \\
1.4+-0.1 \\
1.0 \\
1.1+-0.1 \\
0.5+-0.2 \\
0.2+-0.2 \\
0.4+-0.1\end{array}$ & $\begin{array}{l}176+-9 \\
161+-15 \\
197 \\
181+-10 \\
236+-83 \\
191+-16 \\
179+-85\end{array}$ & $\begin{array}{l}2.7+-0.4 \\
1.8+-0.2 \\
1.1 \\
1.4+-0.3 \\
0.7+-0.2 \\
0.4+-0.0 \\
0.8+-0.3\end{array}$ & $\begin{array}{l}-1.9+-0.5 \\
-1.4+-0.1 \\
-0.9 \\
-1.0+-0.1 \\
+0.2+-0.2 \\
+0.0+-0.0 \\
+0.0+-0.1\end{array}$ & $\begin{array}{l}256+-1 \\
161+-67 \\
161 \\
134+-99 \\
237+-93 \\
202+-7 \\
164+-79\end{array}$ & $\begin{array}{ll}83+- & 7 \\
359+- & 91 \\
315 & \\
305+- & 12 \\
311+- & 11 \\
305+- & 31 \\
317+- & 56\end{array}$ \\
\hline $\begin{array}{l}\mathrm{S} 3 \\
42^{\circ} 45^{-\mathrm{N}} \\
63^{\circ} 30^{-} \mathrm{W}\end{array}$ & $\begin{array}{r}371 \\
97 \\
262\end{array}$ & $\begin{array}{l}230 \\
500 \\
690\end{array}$ & $\begin{array}{r}480 \\
210 \\
20\end{array}$ & $\begin{array}{l}1.5 \\
0.9 \\
1.0\end{array}$ & $\begin{array}{r}259 \\
9 \\
22\end{array}$ & $\begin{array}{l}1.1 \\
0.5 \\
0.3\end{array}$ & $\begin{array}{l}154 \\
186 \\
184\end{array}$ & $\begin{array}{l}1.5 \\
1.1 \\
1.0\end{array}$ & $\begin{array}{l}-1.0 \\
+0.0 \\
+0.1\end{array}$ & $\begin{array}{r}94 \\
188 \\
200\end{array}$ & $\begin{array}{l}292 \\
300 \\
289\end{array}$ \\
\hline $\begin{array}{l}\mathrm{S} 1 \\
42^{\circ} 49^{-} \mathrm{N} . \\
63^{\circ} 30^{-} \mathrm{W} .\end{array}$ & $\begin{array}{l}266 \\
752 \\
180 \\
632 \\
572\end{array}$ & $\begin{array}{r}20 \\
50 \\
100 \\
150 \\
230\end{array}$ & $\begin{array}{r}220 \\
190 \\
140 \\
90 \\
10\end{array}$ & $\begin{array}{l}3.7+-0.2 \\
3.5+-0.3 \\
3.8 \\
3.9+-0.0 \\
4.1+-0.4\end{array}$ & $\begin{array}{lr}249+- & 17 \\
250+- & 5 \\
275 & \\
252+- & 17 \\
257+- & 19\end{array}$ & $\begin{array}{l}2.9+-0.4 \\
2.0+-0.1 \\
2.4 \\
2.3+-0.1 \\
2.7+-0.1\end{array}$ & $\begin{array}{l}167+-13 \\
176+-16 \\
169 \\
159+-21 \\
195+-19\end{array}$ & $\begin{array}{l}3.8+-0.2 \\
3.6+-0.4 \\
3.9 \\
3.9+-0.0 \\
4.4+-0.4\end{array}$ & $\begin{array}{l}-2.8+-0.4 \\
-1.8+-0.3 \\
-2.3 \\
-2.2+-0.1 \\
-2.2+-0.1\end{array}$ & $\begin{array}{lr}202+- & 81 \\
245+- & 8 \\
104 & \\
73+- & 16 \\
245+- & 20\end{array}$ & $\begin{array}{cc}55+- & 92 \\
80+- & 10 \\
285 & \\
273+- & 3 \\
67+- & 1\end{array}$ \\
\hline $\begin{array}{l}\mathrm{S} 6 \\
43^{\circ} 00^{-} \mathrm{N} \\
63^{\circ} 30^{-} \mathrm{W} .\end{array}$ & $\begin{array}{l}250 \\
492 \\
182 \\
380\end{array}$ & $\begin{array}{r}20 \\
50 \\
100 \\
153\end{array}$ & $\begin{array}{r}150 \\
120 \\
70 \\
17\end{array}$ & $\begin{array}{l}5.4 \\
4.5+-0.8 \\
4.1 \\
3.4+-0.1\end{array}$ & $\begin{array}{l}251 \\
260+-14 \\
268 \\
270+-16\end{array}$ & $\begin{array}{l}3.2 \\
2.0+-0.2 \\
2.4 \\
1.6+-0.1\end{array}$ & $\begin{array}{l}170 \\
204+-13 \\
197 \\
214+-16\end{array}$ & $\begin{array}{l}5.4 \\
4.7+-0.8 \\
4.2 \\
3.6+-0.1\end{array}$ & $\begin{array}{l}-3 \cdot 1 \\
-1.6+-0.1 \\
-2.2 \\
-1.3+-0.1\end{array}$ & $\begin{array}{l}247 \\
254+-17 \\
260 \\
264 t-16\end{array}$ & $\begin{array}{l}82 \\
73+- \\
75 \\
73+-\end{array}$ \\
\hline $\begin{array}{l}\mathrm{SS} 13 \\
44^{\circ} 17^{-} \mathrm{N} . \\
63^{\circ} 46^{-} \mathrm{W} .\end{array}$ & $\begin{array}{l}43 \\
52 \\
43\end{array}$ & $\begin{array}{l}14 \\
89 \\
95\end{array}$ & $\begin{array}{r}84 \\
9 \\
3\end{array}$ & $\begin{array}{l}3.1 \\
1.8 \\
2.1\end{array}$ & $\begin{array}{l}247 \\
235 \\
220\end{array}$ & $\begin{array}{r}2.0 \\
.1 .4 \\
2.4\end{array}$ & $\begin{array}{l}193 \\
253 \\
257\end{array}$ & $\begin{array}{l}3.4 \\
2.3 \\
3.0\end{array}$ & $\begin{array}{l}-1.5 \\
+0.3 \\
+1.0\end{array}$ & $\begin{array}{l}235 \\
242 \\
241\end{array}$ & $\begin{array}{l}64 \\
53 \\
40\end{array}$ \\
\hline $\begin{array}{l}\mathrm{SS} 12 \\
44^{\circ} 25^{\circ} \mathrm{N} . \\
63^{\circ} 57^{-\mathrm{W}} .\end{array}$ & $\begin{array}{l}69 \\
70 \\
43\end{array}$ & $\begin{array}{l}14 \\
20 \\
54\end{array}$ & $\begin{array}{r}46 \\
40 \\
6\end{array}$ & $\begin{array}{l}1.6+-1.3 \\
2.1 \\
0.4\end{array}$ & $\begin{array}{l}193+-20 \\
214 \\
32\end{array}$ & $\begin{array}{l}1.4+-0.7 \\
0.6 \\
0.1\end{array}$ & $\begin{array}{l}197+-24 \\
212 \\
208\end{array}$ & $\begin{array}{l}2.2+-1.5 \\
2.2 \\
0.4\end{array}$ & $\begin{array}{l}0.0+-0.0 \\
-0.0 \\
+0.0\end{array}$ & $\begin{array}{l}195+-23 \\
214 \\
212\end{array}$ & $\begin{array}{l}46+-11 \\
74 \\
284\end{array}$ \\
\hline $\begin{array}{l}\mathrm{S} 2 \\
42^{\circ} 46^{\circ} \mathrm{N} . \\
64^{\circ} 00^{\circ} \mathrm{W} .\end{array}$ & $\begin{array}{r}84 \\
334 \\
247\end{array}$ & $\begin{array}{r}30 \\
50 \\
220\end{array}$ & $\begin{array}{r}210 \\
190 \\
20\end{array}$ & $\begin{array}{l}4.8 \\
5.8+-0.2 \\
2.4+-0.5\end{array}$ & $\begin{array}{l}253 \\
286+-20 \\
17+-17\end{array}$ & $\begin{array}{l}4.5 \\
3.6+-0.5 \\
1.3+-0.7\end{array}$ & $\begin{array}{l}184 \\
207+-21 \\
243+-37\end{array}$ & $\begin{array}{l}5.4 \\
5.8+-0.2 \\
2.7+-0.1\end{array}$ & $\begin{array}{l}-3.8 \\
-3.5+-0.4 \\
-0.7+-0.1\end{array}$ & $\begin{array}{l}223 \\
279+-16 \\
205+-10\end{array}$ & $\begin{array}{ll}50 & \\
79+- & 5 \\
294+- & 22\end{array}$ \\
\hline $\begin{array}{l}\mathrm{S} 7 \\
42^{\circ} 42^{-} \mathrm{N} . \\
64^{\circ} 02^{-} \mathrm{W} .\end{array}$ & $\begin{array}{l}338 \\
311\end{array}$ & $\begin{array}{l}230 \\
690\end{array}$ & $\begin{array}{r}480 \\
20\end{array}$ & $\begin{array}{l}0.7 \\
0.4+-0.0\end{array}$ & $\begin{array}{l}339 \\
114+-\quad 1\end{array}$ & $\begin{array}{l}0.8 \\
0.4+-0.1\end{array}$ & $\begin{array}{l}205 \\
257+-18\end{array}$ & $\begin{array}{l}1.0 \\
0.6+-0.1\end{array}$ & $\begin{array}{l}-0.4 \\
+0.2+-0.1\end{array}$ & $\begin{array}{l}186 \\
274+-\quad 7\end{array}$ & $\begin{array}{l}321 \\
318+-\end{array}$ \\
\hline $\begin{array}{l}\mathrm{C} 5 \\
43^{\circ} 34^{-} \mathrm{N} . \\
65^{\circ} 06^{-} \mathrm{W} .\end{array}$ & $\begin{array}{l}170 \\
174 \\
173\end{array}$ & $\begin{array}{l}15 \\
30 \\
50\end{array}$ & $\begin{array}{l}45 \\
30 \\
10\end{array}$ & $\begin{array}{l}2.7+-1.4 \\
5.9 \\
4.5\end{array}$ & $\begin{array}{l}225+-29 \\
171 \\
351\end{array}$ & $\begin{array}{l}1.1+-0.7 \\
3.0 \\
0.7\end{array}$ & $\begin{array}{l}95+-34 \\
30 \\
30\end{array}$ & $\begin{array}{l}2.8 \\
6.4 \\
4.5\end{array}$ & $\begin{array}{l}-0.8 \\
-1.7 \\
+0.5\end{array}$ & $\begin{array}{r}50 \\
358 \\
351\end{array}$ & $\begin{array}{r}286 \\
293 \\
83\end{array}$ \\
\hline
\end{tabular}

Instrument type, method of analysis and source of data are listed alphabetically by stations in Table 3 . If UMINOR $<0$, ellipse rotates clockwise 
Table 9. $O_{1}$ tidal current parameters-Continued

Table 9. 01 TIDAL CURRENT PARAMETERS--Cont Inued

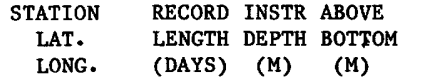

FOURIER
EAST PHASE COEFF ICIENTS $(\mathrm{CM} / \mathrm{SEC}) \quad(\mathrm{DEG}-\mathrm{G}) \quad(\mathrm{CM} / \mathrm{SEC}) \quad(\mathrm{DEG}-\mathrm{G})$

$\begin{array}{cc}\text { CURRENT } & \text { ELLIPSE } \\ \text { UMAJOR } & \text { UMINOR } \\ \text { (CM/SEC) } & \text { (CM/SEC) }\end{array}$

PARAMETERS

PHASE ORIEN (DEG-G) (DEG-TRUE)

SCOTIAN SHELF--CONT.

C1

\begin{tabular}{|c|c|c|}
\hline 1 & 162 & 15 \\
\hline $43^{\circ} 11^{-} \mathrm{N}$. & 162 & 30 \\
\hline $65^{\circ} 43^{- \text {พ. }}$ & 174 & 50 \\
\hline & 161 & 15 \\
\hline $42^{\circ} 50^{-} \mathrm{N}$ & 103 & 50 \\
\hline $65^{\circ} 50^{\circ} \mathrm{W}$. & 161 & 100 \\
\hline
\end{tabular}

NORTHEAST CHANNEL

$\begin{array}{lrrr}\text { NEC1 } & 174 & 103 & 120 \\ 42^{\circ} 20^{\circ} \mathrm{N} . & 174 & 153 & 70 \\ 65^{\circ} 55^{-\mathrm{W}} . & 174 & 207 & 16 \\ \mathrm{NEC2}^{*} & & & \\ 42^{\circ} 18^{-\mathrm{N}} . & 174 & 106 & 134 \\ 65^{\circ} 58^{-\mathrm{W}} . & 58 & 217 & 84 \\ \mathrm{NEC3}^{*} & 87 & 112 & 116 \\ 42^{\circ} 11^{-\mathrm{N}} . & 87 & 162 & 66 \\ 66^{\circ} 02^{-\mathrm{W}} . & 174 & 220 & 16\end{array}$

GULF OF MAINE

$\begin{array}{crrr}\text { CASHES LEDGE } & 58 & 33 & 157 \\ 43^{\circ} 11^{-N} \text {. } & 58 & 68 & 122 \\ 69^{\circ} 05^{-} \mathrm{W} . & 58 & 180 & 10 \\ & & & \\ \text { MONHEGAN } & 57 & 33 & 65 \\ 43^{\circ} 40^{-} \mathrm{N} . & 58 & 68 & 30 \\ 69^{\circ} 23^{-} \mathrm{W} . & & & \\ & & & \\ \text { C. PORPOISE } & 74 & 33 & 65 \\ 43^{\circ} 13^{-N} \mathrm{~N} . & 74 & 68 & 30 \\ 70^{\circ} 17^{-} \mathrm{W} . & & & \\ \text { BOSTON L.S. } & 369 & 2 & 3 \\ 42^{\circ} 20^{-} \mathrm{N} . & & & \\ 70^{\circ} 45^{-} \mathrm{W} . & & & \end{array}$

$\begin{array}{lllll}1.4 & 334 & 0.3 & 106 & 1.4\end{array}$

$0.5+-0.2 \quad 342+-32$

$0.7+-0.3 \quad 310+-26$

$0.3+-0.3 \quad 22+-52$

$0.2+-0.1 \quad 73+-32$

$\begin{array}{lr}0.7 & 339 \\ 0.3+-0.1 & 4+-11\end{array}$

0.2

261

$0.2+-0.8 \quad 154+-27$

$0.3+-0.1 \quad 231+-24$

$0.4+-0.2 \quad 349+-35$

$0.6+-0.3 \quad 18+-27$

$0.3+-0.2 \quad 326+-34$

$0.1 \quad 166$

$0.4 \quad 310$

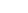

BAY OF FUNDY

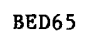

$45^{\circ} 08^{\circ} \mathrm{N}$ $65^{\circ} 08^{-} \mathrm{W}$.

BED66

$45^{\circ} 25^{-N}$ $65^{\circ} 07^{-} \mathrm{W}$.

$$
29 \mathrm{c} \quad 25 \quad 37 \mathrm{a} \quad 1.4
$$

86

0.9

77
$6.1+-1.2 \quad 276+-11$

$4.6+-0.7$ 290t- 8 0.7+-0.1 285+- 11

$4.9+-0.9 \quad 311+-11$

$\begin{array}{lllll}4.4+-0.5 & 315+- & 6 & 4.9+-0.8 & 146+-10\end{array}$

$3.6+-0.7 \quad 308+-11 \quad 1.2+-0.3 \quad 303+-12$
6.2

6.3

4.7

5.0

7.3

3.8

$3.3+-0.3$

$2.5+-0.4$

$2.6+-0.4$

$2.4+-0.4$ 270t- $23 \quad 1.5+-0.6 \quad 354+-47$

$2.4+-0.3 \quad 263+-14 \quad 1.9+-0.5 \quad 334+-24$

$2.8+-1.2 \quad 295+-19$

$2.3+-0.5 \quad 267+-10$

$2.0+-0.6 \quad 231+-14$

$1.4+-0.8 \quad 316+-8$

$1.5+-0.3 \quad 352+-30$

$2.3+-1.5 \quad 338+-17$

$1.3+-0.6 \quad 289+-25$

$2.1+-0.5 \quad 266+-13$

$0.9+-0.2 \quad 20+-29$

$0.5+-0.1 \quad 73+-31$

$0.7+-0.3 \quad 20+-47$

$3.1+-1.4$

$2.1+-0.5$
$-1.4$

$-1.7$

$-0.1$

-1.6
-0.7

$-0.1$

319

307

274
279
90
308
319
307

1.6

$-0.1$

83

$+0.2+-0.5$

$+1.2+-0.5$

$+1.4+-0.4$

$303+-18$

$282+-26$

$272+-22$

$+0.3+-0.3$

$298+-16$

$270+-17$

$+1.2+-0.0 \quad 206+-47$

206+- 47

$+0.6+-0.4$

$287+-40$

$266+-12$

$262+-46$

84
114
81
79
132
72 
Table 9. $\mathrm{O}_{1}$ tidal current parameters-Continued

Table 9. Ol TIDAL CURRENT PARAMETERS--Continued

\begin{tabular}{|c|c|c|c|c|c|c|c|c|c|c|c|}
\hline STATION & RECORD & INSTR & ABOVE & & OURIER & COEFFICIENTS & & CUI & ELLIPSE & PARAMETERS & \\
\hline $\begin{array}{l}\text { LAT. } \\
\text { LONG. }\end{array}$ & $\begin{array}{l}\text { LENGTH } \\
\text { (DAYS) }\end{array}$ & $\begin{array}{l}\text { DEPTH } \\
\text { (M) }\end{array}$ & $\begin{array}{l}\text { вотTOM } \\
\text { (M) }\end{array}$ & $\begin{array}{c}\text { EAST } \\
(\mathrm{CM} / \mathrm{SEC})\end{array}$ & $\begin{array}{c}\text { PHASE } \\
(\text { DEG-G) }\end{array}$ & $\begin{array}{c}\text { NORTH } \\
(\mathrm{CM} / \mathrm{SEC})\end{array}$ & $\begin{array}{r}\text { PHASE } \\
\text { (DEG-G) }\end{array}$ & $\begin{array}{r}\text { UMAJOR } \\
\text { (CM/SEC) }\end{array}$ & $\begin{array}{l}\text { UMINOR } \\
\text { (CM/SEC) }\end{array}$ & $\begin{array}{c}\text { PHASE } \\
\text { (DEG-G) }\end{array}$ & $\begin{array}{c}\text { ORIEN } \\
\text { (DEG-TRUE) }\end{array}$ \\
\hline
\end{tabular}

GULF OF MAINE--CONT.

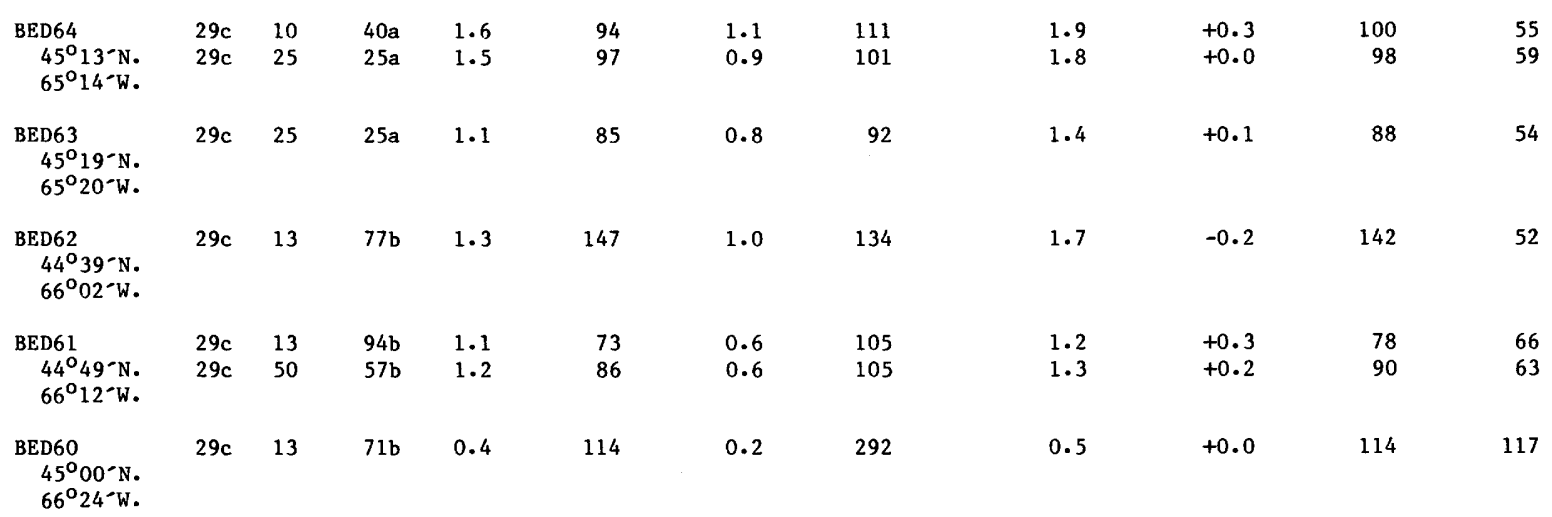

GEORGES BANK

\begin{tabular}{|c|c|c|c|c|c|c|c|c|c|c|c|c|}
\hline $\begin{array}{l}41^{\circ} 42^{-N} . \\
66^{\circ} 36^{\prime} \mathrm{W} .\end{array}$ & 145 & 51 & 15 & $1.6+-0.5$ & $110+-12$ & $2.5+-0.6$ & $28+-13$ & $2.6+-0.6$ & $-1 \cdot 5+-0.5$ & $33+-$ & 21 & $9+-$ \\
\hline $\begin{array}{l}42^{\circ} 12^{-N} . \\
66^{\circ} 41^{-} \mathrm{w} .\end{array}$ & $\begin{array}{l}29 \\
29\end{array}$ & $\begin{array}{r}79 \\
129\end{array}$ & $\begin{array}{r}140 \\
90\end{array}$ & $\begin{array}{l}1.2 \\
2.1\end{array}$ & $\begin{array}{l}294 \\
276\end{array}$ & $\begin{array}{l}0.9 \\
0.9\end{array}$ & $\begin{array}{l}15 \\
21\end{array}$ & $\begin{array}{l}1.2 \\
2.1\end{array}$ & $\begin{array}{l}+0.9 \\
+0.8\end{array}$ & $\begin{array}{l}305 \\
274\end{array}$ & & $\begin{array}{l}75 \\
97\end{array}$ \\
\hline $\begin{array}{l}42^{\circ} 02-\mathrm{N} . \\
66^{\circ} 41^{-W} \text {. }\end{array}$ & $\begin{array}{l}29 \\
29\end{array}$ & $\begin{array}{l}19 \\
44\end{array}$ & $\begin{array}{l}52 \\
27\end{array}$ & $\begin{array}{l}0.8 \\
1.2\end{array}$ & $\begin{array}{r}82 \\
277\end{array}$ & $\begin{array}{l}3.2 \\
1.8\end{array}$ & $\begin{array}{l}46 \\
18\end{array}$ & $\begin{array}{l}3.3 \\
1.8\end{array}$ & $\begin{array}{l}-0.5 \\
+1.2\end{array}$ & $\begin{array}{l}48 \\
26\end{array}$ & & $\begin{array}{r}12 \\
347\end{array}$ \\
\hline $\begin{array}{l}41^{\circ} 53-\mathrm{N} . \\
66^{\circ} 41^{-} \mathrm{W} .\end{array}$ & $\begin{array}{l}58 \\
58 \\
58\end{array}$ & $\begin{array}{l}11 \\
26 \\
36\end{array}$ & $\begin{array}{l}59 \\
44 \\
34\end{array}$ & $\begin{array}{l}0.3+-0.2 \\
0.2+-0.0 \\
0.4+-0.0\end{array}$ & $\begin{array}{r}224+-122 \\
64+-13 \\
109+-30\end{array}$ & $\begin{array}{l}1.6+-0.4 \\
1.8+-0.5 \\
1.8+-0.4\end{array}$ & $\begin{array}{rr}7+- & 1 \\
20+- & 4 \\
37+- & 3\end{array}$ & $\begin{array}{l}1.6+-0.4 \\
1.8+-0.5 \\
1.9+-0.4\end{array}$ & $\begin{array}{l}-0.1+-0.3 \\
-0.1+-0.1 \\
-0.4+-0.1\end{array}$ & $\begin{array}{r}5+- \\
21+- \\
37+-\end{array}$ & $\begin{array}{l}4 \\
4 \\
4\end{array}$ & $\begin{array}{r}354+- \\
5+- \\
3+-\end{array}$ \\
\hline $\begin{array}{l}* \\
40^{\circ} 56^{-} \mathrm{N} . \\
66^{\circ} 58^{-} \mathrm{W} .\end{array}$ & $\begin{array}{l}58 \\
58 \\
58\end{array}$ & $\begin{array}{l}10 \\
36 \\
69\end{array}$ & $\begin{array}{r}67 \\
41 \\
8\end{array}$ & $\begin{array}{l}2.1 \\
2.6 \\
0.8\end{array}$ & $\begin{array}{l}129 \\
169 \\
183\end{array}$ & $\begin{array}{l}0.2 \\
2.1 \\
1.9\end{array}$ & $\begin{array}{r}55 \\
100 \\
92\end{array}$ & $\begin{array}{l}2.1 \\
2.8 \\
1.9\end{array}$ & $\begin{array}{l}-0.2 \\
-1.8 \\
-0.8\end{array}$ & $\begin{array}{r}129 \\
151 \\
92\end{array}$ & & $\begin{array}{r}89 \\
62 \\
0\end{array}$ \\
\hline $\begin{array}{l}42^{\circ} 12^{-N} . \\
67^{\circ} 15^{-} \mathrm{W} .\end{array}$ & $\begin{array}{l}15 \\
15 \\
15\end{array}$ & $\begin{array}{l}30 \\
40 \\
75\end{array}$ & $\begin{array}{l}173 \\
163 \\
128\end{array}$ & $\begin{array}{l}3.1 \\
2.9 \\
1.5\end{array}$ & $\begin{array}{l}345 \\
348 \\
323\end{array}$ & $\begin{array}{l}0.9 \\
0.7 \\
0.7\end{array}$ & $\begin{array}{l}176 \\
271 \\
321\end{array}$ & $\begin{array}{l}3.2 \\
2.9 \\
1.7\end{array}$ & $\begin{array}{l}-0.2 \\
-0.7 \\
-0.0\end{array}$ & $\begin{array}{l}346 \\
347 \\
323\end{array}$ & & $\begin{array}{r}106 \\
87 \\
65\end{array}$ \\
\hline
\end{tabular}

Instrument type, method of analysis and source of data are listed alphabetically by stations in Table 3.

If UMINOR $<0$, ellipse rotates clockwise

a - water depth estimated from DMA chart 14040 (old 609)

b - water depth estimated from DMA chart 13102

c - estimated to be at least 29-days

* - depths have been calculated from the mean pressure and error estimates are taken from across-isobath and along-isobath directions since these are nearly the same as the ellipse axis. These records span identical time periods. 
Table 9. $\mathrm{O}_{1}$ tidal current parameters-Continued

Table 9. Ol TIDAL CURRENT PARAMETERS-Continued

$\begin{array}{cl}\text { STATION } & \text { RECORD INSTR ABOVE } \\ \text { LAT. } & \text { LENGTH DEPTH BOTTOY }\end{array}$

LONG.

(DAYS) (H) (M)

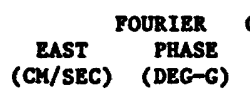

COEFFICIENTS
NORTH

(CH/SEC)
PHASE

(DEG-G)

$\begin{array}{cccc}\text { CURRENT ELLIPSE } & \text { PARAMETERS } \\ \text { UMUJOR } & \text { UMIROR } & \begin{array}{c}\text { PHASE } \\ \text { ORIER }\end{array} \\ (\mathrm{CH} / \mathrm{SEC}) & (\mathrm{CH} / \mathrm{SEC}) & \text { (DEG-G) } & \text { (DEG-TRUE) }\end{array}$

GEORGES BANK-CONT.

$$
\begin{aligned}
& \left.42^{\circ} 03^{\prime}\right) \\
& 67^{\circ} 15^{\prime} \mathrm{H}
\end{aligned}
$$$$
\text { P3 }
$$

$\begin{array}{ll}P 3 & 15 \\ 41^{\circ} 53 \% \mathrm{~N} . & 15 \\ 67^{\circ} 15^{\circ} \mathrm{W} . & 15\end{array}$

$$
\mathrm{MB}
$$$$
41^{\circ} 20^{\prime}
$$

$\begin{array}{ll}10 & 54 \\ 15 & 45 \\ 34 & 30 \\ 58 & 10 \\ 60 & \end{array}$
$41^{\circ} 59 \cdot \mathrm{N}$. $87 \quad 25$ $67^{\circ} 47^{\prime} \mathrm{H}$

great SOUTH CRANAEI $145 \quad 10-\mathrm{B} \quad 56$ $10^{\circ} 51$ 'N. $171 \quad 10-\mathrm{R} \quad 56$ $68^{\circ} 49^{\circ} \mathrm{W} .116$ 51-H 15 51-R 15 $40^{\circ} 49^{\prime} \mathrm{R}$. $87 \quad 58 \quad 20$ $69^{\circ} 00^{\prime} \mathrm{W}$.
$1.7+0.0321+10 \quad 1.6+-0.4319+4$

$1.6 \quad 323$

0.8 347

$2.3 \quad 305$

$\begin{array}{ll}1.5 & 307 \\ 5.8 & 224\end{array}$

$1.0 \quad 252$

$\begin{array}{ll}1.2 & 312 \\ 1.8 & 142\end{array}$

2.4

163

$0.6 \quad 128$

1.0

78

$1.8+-0.9 \quad 140+53$

$1.6+0.6 \quad 130 t 33$

$1.3+0.4 \quad 131+36$

$1.9+0.7 \quad 54+31$

$1.5+0.5 \quad 59+31$

$1.2+0.5 \quad 69+33$

$1.0+0.3 \quad 121+28$

$1.1+0.4$

$61+17$

$1.7+0.4 \quad 78+6 \quad 2.3+0.3 \quad 28+3$

$1.3+0.5 \quad 128+15$

$1.8+1.1 \quad 110+24$

$1.3+0.5128+15$

$1.7+0.5 \quad 141+18$

$1.4 t-0.3 \quad 140 t-30$

$1.5+0.5130+18$

$1.7+0.8 \quad 56+2$

$1.7+1.158+43$

$1.7+0.8 \quad 56+2$

$1.9+0.366+10$

$1.5+-0.553+19$

$1.4+0.2 \quad 354+10$

$1.4+0.4 \quad 354 t-11$
$1.4+0.1 \quad 75+25$
(CE/SEC)

(Cx/SEC)

$\begin{array}{lllll}2.3+-0.3 & -0.1+-0.1 & 320+ & 7 & 47+7 \\ 1.8 & +0.3 & 327 & & 66\end{array}$

$2.4 \quad-0.6 \quad 300$

$1.9+0.1 \quad 309$

$5.8-1.7 \quad 223$

2.5

$-2.1$

1.1

$2.4+-1.0$

$1.9+0.6$

$1.6+0.4$

$1.3+0.4$

$2.6+0.3$

$2.0+-0.4$

$2.2 t-1.0$

$2.0+0.4$

$2.1+0.4$

$1.7+-0.2$

$1.7+0.5$

$2.0+0.3$
$-0.4$

$-1.1+-0.7$

$-1.2+-0.5$

$-0.8+0.5$

$-0.7+0.3$

$-1.1+0.2$

$-0.9+-0.2$

$-1.1+-1.1$

$-0.9+0.2$

$-1.4 t-0.3$

$-1.1+0.2$

$-1.3+-0.5$

$-0.2+-0.3$
189

73

52
87

30

86

22

$\begin{array}{rr}68+41 & 7+49 \\ 100+41 & 66+66 \\ 96+51 & 39+50 \\ 89+22 & 43+10 \\ 43+4 & 32+9\end{array}$

96+ $20 \quad 57+26$

$98+51 \quad 49+49$

$94+36 \quad 34 t-26$

$105+22 \quad 42+9$

95t $36 \quad 54 t 33$

$352+845+11$
$98+51 \quad 49+49$

Instrunent type, wethod of analye1s and source of data are lieted alphabetically by statlone in Table 3.

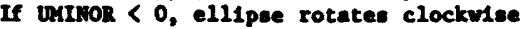

- depths have been calculated from the wean pressure and error estiates are taken from acrose-1sobath and along-1sobath directione ince these are nearly the eame as the ellipse axis. These records span identical tiae periods.

wh- tripod and subsurface woorings were at different deptha. 
Table 9. $\mathrm{O}_{1}$ tidal current parameters-Continued

Table 9. 01 TIDAL CURRENT PARAMETERS--Continued

\begin{tabular}{|c|c|c|c|c|c|c|c|c|c|c|}
\hline STATION & RECORD & INSTR & ABOVE & & OURIER & COEFFICIENTS & & CUF & ENT ELLIPSE & PARAMETERS \\
\hline $\begin{array}{l}\text { LAT. } \\
\text { LONG. }\end{array}$ & $\begin{array}{l}\text { LENGTH } \\
\text { (DAYS) }\end{array}$ & $\begin{array}{l}\text { DEPTH } \\
\text { (M) }\end{array}$ & $\begin{array}{l}\text { воттом } \\
\text { (M) }\end{array}$ & $\begin{array}{c}\text { EAST } \\
(\mathrm{CM} / \mathrm{SEC})\end{array}$ & $\begin{array}{l}\text { PHASE } \\
(\text { DEG -G) }\end{array}$ & $\begin{array}{c}\text { NORTH } \\
\text { (CM/SEC) }\end{array}$ & $\begin{array}{r}\text { PHASE } \\
(\mathrm{DEG}-G)\end{array}$ & $\begin{array}{r}\text { UMAJOR } \\
\text { (CM/SEC) }\end{array}$ & $\begin{array}{r}\text { UMINOR } \\
\text { (CM/SEC) }\end{array}$ & $\begin{array}{cc}\text { PHASE } & \text { ORIEN } \\
(\text { DEG }-G) & \text { (DEG-TRUE) }\end{array}$ \\
\hline
\end{tabular}

GREAT SOUTH CHANNEL-CONT.

\begin{tabular}{|c|c|c|c|c|c|c|c|c|c|c|c|c|}
\hline GSC2 & 109 & 10 & 73 & $3.5+-0.8$ & $102+-14$ & $6.6+-1.1$ & $77+-$ & 9 & 7.4 & $-1 \cdot 3$ & 82 & 27 \\
\hline $40^{\circ} 51-\mathrm{N}$ & 152 & 42 & 41 & $3.2+-0.5$ & $96+-10$ & $6.4+-0.6$ & $80+-$ & 6 & 7.1 & -0.8 & 83 & 26 \\
\hline $69^{\circ} 01-\mathrm{W}$. & 152 & 76 & 7 & $3.1+-0.5$ & $273+-10$ & $7 \cdot 1+-1 \cdot 2$ & $295+-$ & 9 & 7.7 & +1.1 & 292 & 23 \\
\hline $\begin{array}{l}\mathrm{N} \\
40^{\circ} 51^{-\mathrm{N}} . \\
69^{\circ} 01^{-\mathrm{W}} .\end{array}$ & 145 & 68 & 15 & $2.4+-0.8$ & $106+-19$ & $3.6+-1.0$ & $74+-$ & 6 & $4.2+-1.2$ & $-1.1+-0.5$ & $33+-11$ & $31+-$ \\
\hline $\begin{array}{l}\mathrm{R} \\
40^{\circ} 30^{-} \mathrm{N} . \\
69^{\circ} 07^{-\mathrm{W}} .\end{array}$ & 116 & 79 & 1 & $2.1+-0.8$ & $113+-23$ & $1.6+-0.3$ & $68+-$ & 28 & $2.5+-0.6$ & $-0.9+-0.3$ & $97+-18$ & $52+-18$ \\
\hline $\begin{array}{l}\mathrm{GSC} 1 \\
40^{\circ} 52^{-} \mathrm{N} .\end{array}$ & $\begin{array}{l}149 \\
149\end{array}$ & $\begin{array}{l}27 \\
49\end{array}$ & $\begin{array}{l}37 \\
15\end{array}$ & $\begin{array}{l}3.9+-0.5 \\
3.9+-0.5\end{array}$ & $\begin{array}{ll}81+- & 7 \\
80+- & 7\end{array}$ & $\begin{array}{l}5.6+-0.6 \\
4.0+-0.5\end{array}$ & $\begin{array}{l}80+- \\
79+-\end{array}$ & $\begin{array}{l}6 \\
7\end{array}$ & $\begin{array}{l}6.8 \\
5.6\end{array}$ & $\begin{array}{l}-0.1 \\
-0.0\end{array}$ & $\begin{array}{l}80 \\
80\end{array}$ & $\begin{array}{l}35 \\
44\end{array}$ \\
\hline
\end{tabular}

LYDONIA CANYON*

\begin{tabular}{|c|c|c|c|c|c|c|c|c|c|c|c|}
\hline $\begin{array}{l}\mathrm{LCA} \\
40^{\circ} 34^{-} \mathrm{N} . \\
67^{\circ} 45^{-} \mathrm{W} .\end{array}$ & $\begin{array}{r}116 \\
58\end{array}$ & $\begin{array}{l}80 \\
99\end{array}$ & $\begin{array}{r}20 \\
1\end{array}$ & $\begin{array}{l}2.0+-0.9 \\
1.0+-0.0\end{array}$ & $\begin{array}{rr}126+- & 25 \\
178+- & 0\end{array}$ & $\begin{array}{l}1.8+-0.6 \\
1.0+-0.2\end{array}$ & $\begin{array}{r}45+-26 \\
107+-\quad 4\end{array}$ & $\begin{array}{l}2.1+-0.9 \\
1.1+-0.1\end{array}$ & $\begin{array}{l}-1.7+-0.6 \\
-0.8+-0.1\end{array}$ & $\begin{array}{l}103+-17 \\
144+-17\end{array}$ & $\begin{array}{l}63+-20 \\
48+-16\end{array}$ \\
\hline $\begin{array}{l}\mathrm{LCB} \\
40^{\circ} 32-\mathrm{N} . \\
67^{\circ} 43^{-} \mathrm{W} .\end{array}$ & $\begin{array}{l}145 \\
145 \\
116\end{array}$ & $\begin{array}{r}92 \\
227 \\
277\end{array}$ & $\begin{array}{r}190 \\
55 \\
5\end{array}$ & $\begin{array}{l}1.5+-0.5 \\
0.4+-0.2 \\
0.5+-0.2\end{array}$ & $\begin{array}{l}135+-22 \\
202+-42 \\
348+-94\end{array}$ & $\begin{array}{l}1.5+-0.3 \\
0.4+-0.3 \\
0.3+-0.4\end{array}$ & $\begin{array}{r}29+-40 \\
126+-80 \\
33+-71\end{array}$ & $\begin{array}{l}1.8+-0.5 \\
0.6+-0.2 \\
0.6+-0.4\end{array}$ & $\begin{array}{l}-1.1+-0.3 \\
-0.1+-0.2 \\
+0.0+-0.1\end{array}$ & $\begin{array}{r}344+-64 \\
128+-81 \\
27+-77\end{array}$ & $\begin{array}{r}319+-36 \\
8+-57 \\
8+-75\end{array}$ \\
\hline $\begin{array}{l}\mathrm{LCC} \\
40^{\circ} 29^{-} \mathrm{N} . \\
67^{\circ} 44^{-} \mathrm{W} .\end{array}$ & 116 & 134 & 50 & $1.0+-0.4$ & $131+-62$ & $0.8+-0.5$ & $74+-31$ & $1.2+-0.6$ & $-0.3+-0.3$ & $80+-47$ & $35+-60$ \\
\hline $\begin{array}{l}\mathrm{LCD} \\
\quad 40^{\circ} 29^{\prime} \mathrm{N} . \\
67^{\circ} 41^{-} \mathrm{W} .\end{array}$ & 116 & 143 & 50 & $1.0+-0.7$ & $123+-18$ & $0.6+-0.5$ & $17+-36$ & $1.0+-0.7$ & $-0.4+-0.4$ & $351+-75$ & $21+-67$ \\
\hline $\begin{array}{l}\mathrm{LCE} \\
\quad 40^{\circ} 25^{-} \mathrm{N} . \\
67^{\circ} 40^{-} \mathrm{W} .\end{array}$ & $\begin{array}{l}145 \\
145 \\
145\end{array}$ & $\begin{array}{l}116 \\
216 \\
595\end{array}$ & $\begin{array}{r}484 \\
384 \\
5\end{array}$ & $\begin{array}{l}1.4+-0.5 \\
0.4+-0.2 \\
0.5+-0.4\end{array}$ & $\begin{array}{r}111+-36 \\
113+-118 \\
45+-81\end{array}$ & $\begin{array}{l}1.3+-0.6 \\
1.2+-0.6 \\
1.3+-0.8\end{array}$ & $\begin{array}{l}23+-65 \\
89+-78 \\
42+-101\end{array}$ & $\begin{array}{l}1.7+-0.8 \\
1.2+-0.6 \\
1.4+-0.9\end{array}$ & $\begin{array}{l}-0.8+-0.5 \\
-0.2+-0.3 \\
+0.2+-0.3\end{array}$ & $\begin{array}{l}77+-92 \\
89+-78 \\
36+-102\end{array}$ & $\begin{array}{r}18+-55 \\
6+-18 \\
16+-10\end{array}$ \\
\hline $\begin{array}{l}\mathrm{LCF} \\
\quad 40^{\circ} 21^{-\mathrm{N}} . \\
67^{\circ} 39^{-} \mathrm{W} .\end{array}$ & $\begin{array}{l}145 \\
174\end{array}$ & $\begin{array}{l}205 \\
405\end{array}$ & $\begin{array}{l}300 \\
100\end{array}$ & $\begin{array}{l}0.8+-0.5 \\
0.6+-0.4\end{array}$ & $\begin{array}{r}52+-11 \\
120+-45\end{array}$ & $\begin{array}{l}0.7+-0.4 \\
0.5+-0.2\end{array}$ & $\begin{array}{r}73+-53 \\
126+-72\end{array}$ & $\begin{array}{l}1.0+-0.5 \\
0.7+-0.4\end{array}$ & $\begin{array}{l}-0.0+-0.5 \\
+0.1+-0.4\end{array}$ & $\begin{array}{r}60+-33 \\
126+-63\end{array}$ & $\begin{array}{l}45+-17 \\
47+-25\end{array}$ \\
\hline $\begin{array}{l}\text { LCG } \\
\quad 40^{\circ} 21^{-N} . \\
67^{\circ} 42^{-} \mathrm{W} .\end{array}$ & $\begin{array}{l}174 \\
174\end{array}$ & $\begin{array}{l}195 \\
395\end{array}$ & $\begin{array}{l}300 \\
100\end{array}$ & $\begin{array}{l}0.7+-0.4 \\
0.4+-0.1\end{array}$ & $\begin{array}{r}148+-81 \\
58+-50\end{array}$ & $\begin{array}{l}0.6+-0.3 \\
0.4+-0.3\end{array}$ & $\begin{array}{r}147+-89 \\
7+-98\end{array}$ & $\begin{array}{l}0.9+-0.4 \\
0.6+-0.2\end{array}$ & $\begin{array}{l}-0.1+-0.4 \\
-0.0+-0.1\end{array}$ & $\begin{array}{r}144+-84 \\
14+-98\end{array}$ & $\begin{array}{l}50+-17 \\
16+-6\end{array}$ \\
\hline
\end{tabular}

Instrument type, method of analysis and source of data are listed alphabetically by stations in Table 3.

If UMINOR $<0$, ellipse rotates clockwise

* - Observations from stations LCB (except 277m), LCE, LCH(except 1375 and 1454m), LCK, LCJ(except $454 \mathrm{~m}$ ), and LCN all begin at 0100 on Dec. 2, 1980 and end at 0100 on Apri1 26, 1981. Observations at LCA $(80 \mathrm{~m}), \mathrm{LCB}(277 \mathrm{~m}), \mathrm{LCJ}(83 \mathrm{~m}), \mathrm{LCK}(454 \mathrm{~m})$ and LCL begin at 0100 on Dec. 2, 1980 but end 29 days earlier on March 28, 1981 . 
Table 9. $\mathrm{O}_{1}$ tidal current parameters-Continued

Table 9. 01 TIDAL CURRENT PARAMETERS--Continued

STATION RECORD INSTR ABOVE

LAT. LENGTH DEPTH BOTTOM

LONG. (DAYS) (M) (M)

LYDONIA CANYON--CONT.

\begin{tabular}{|c|c|c|}
\hline $\begin{array}{l}\mathrm{LCH}^{*} \\
40^{\circ} 18-\mathrm{N} . \\
67^{\circ} 40^{-} \mathrm{W} .\end{array}$ & $\begin{array}{r}145 \\
145 \\
145 \\
29 \\
87\end{array}$ & $\begin{array}{r}290 \\
540 \\
890 \\
1454 \\
1375\end{array}$ \\
\hline $\begin{array}{l}\mathrm{LCI} \\
\qquad \begin{array}{l}40^{\circ} 23^{-} \mathrm{N} \\
67^{\circ} 33^{-} \mathrm{W}\end{array}\end{array}$ & $\begin{array}{l}145 \\
145 \\
145 \\
145\end{array}$ & $\begin{array}{r}10 \\
55 \\
195 \\
245\end{array}$ \\
\hline $\begin{array}{l}\text { LCJ } \\
\qquad 0^{\circ} 21-\mathrm{N} . \\
67^{\circ} 32^{-} \mathrm{W} .\end{array}$ & $\begin{array}{l}116 \\
145 \\
145\end{array}$ & $\begin{array}{r}83 \\
223 \\
471\end{array}$ \\
\hline $\begin{array}{l}\text { LCK } \\
\qquad 40^{\circ} 16^{-} \mathrm{N} . \\
67^{\circ} 47^{-} \mathrm{W} .\end{array}$ & $\begin{array}{l}145 \\
145\end{array}$ & $\begin{array}{l}204 \\
454\end{array}$ \\
\hline $\begin{array}{l}\text { LCL } \\
40^{\circ} 32^{-} \mathrm{N} . \\
67^{\circ} 36^{-} \mathrm{W} .\end{array}$ & $\begin{array}{l}116 \\
116\end{array}$ & $\begin{array}{r}65 \\
105\end{array}$ \\
\hline $\begin{array}{l}\mathrm{LCM}^{\star \star} \\
\quad 40^{\circ} 30^{-} \mathrm{N} \\
67^{\circ} 49^{-} \mathrm{W}\end{array}$ & $\begin{array}{l}145 \\
174\end{array}$ & $\begin{array}{l}103 \\
119\end{array}$ \\
\hline $\begin{array}{l}\mathrm{LCN} \\
\quad 40^{\circ} 21^{-} \mathrm{N} . \\
67^{\circ} 40^{-} \mathrm{W} .\end{array}$ & $\begin{array}{l}145 \\
145\end{array}$ & $\begin{array}{l}243 \\
841\end{array}$ \\
\hline
\end{tabular}

$\begin{array}{cccr} & \text { FOURIER } & \text { COEFFICIENTS } & \\ \text { EAST } & \text { PHASE } & \text { NORTH } & \text { PHASE } \\ (\mathrm{CM} / \mathrm{SEC}) & (\text { DEG }-\mathrm{G}) & (\mathrm{CM} / \mathrm{SEC}) & (\text { DEG -G) }\end{array}$

$0.8+-0.5 \quad 200+-64 \quad 0.8+-0.1 \quad 32+-100$

$\begin{array}{llll}0.6+-0.3 & 228+-67 & 0.5+-0.4 & 106+-73\end{array}$

$0.2+-0.1 \quad 98+-78 \quad 0.5+-0.2 \quad 316+-83$

0.2

176

$\begin{array}{ll}0.5+-0.2 & 316+-83 \\ 0.3 & 285 \\ 0.4+-0.3 & 100+-76\end{array}$

$0.3+-0.2168+-93$

$0.4+-0.3 \quad 100+-76$

$2.0+-1.1 \quad 102+-80$

$1.1+-0.6 \quad 80+-87$

$0.8+-0.2 \quad 150+-55$

$1.5+-0.9 \quad 329+-68$

$1.1+-0.5 \quad 63+-72$

$0.7+-0.4 \quad 39+-56$

$0.6+-0.4 \quad 18+-89 \quad 0.6+-0.5 \quad 71+-59$

$1.2+-0.4 \quad 127+-66$

$0.6+-0.5156+-81$

$1.0+-0.6 \quad 332+-66$

$0.5+-0.2$. $42+-42$

$0.6+-0.3 \quad 195 t-76 \quad 0.4 t-0.3 \quad 75 t-66$

$0.5+-0.4 \quad 137+-64$

$0.9+-0.5 \quad 271+-65$

$0.4+-0.4 \quad 50+-74$

$2.0+-0.1 \quad 115+-38 \quad 1.7+-0.7 \quad 45+-24$

$1.4+-0.5 \quad 139+-59 \quad 1.5+-0.5 \quad 71+-36$

$1.5+-0.6 \quad 128+-20 \quad 1.3+-0.5 \quad 45+-44$

$0.9+-0.5 \quad 148+-45 \quad 1.3+-0.7 \quad 74+-34$

$1.0+-0.4 \quad 54+-84$

$0.4+-0.3299+-76$

$0.6+-0.2 \quad 87+-89$

$0.5+-0.3 \quad 328+-70$
$0.7+-0.2 \quad 306+-71$

\begin{tabular}{cccc}
\multicolumn{2}{c}{ CURRENT ELLIPSE } & PARAMETERS \\
UMAJOR & UMINOR & PHASE & ORIEN \\
$(\mathrm{CM} / \mathrm{SEC})$ & $(\mathrm{CM} / \mathrm{SEC})$ & $(\mathrm{DEG}-\mathrm{G})$ & $(\mathrm{DEG}-\mathrm{TRUE})$
\end{tabular}

$(\mathrm{CM} / \mathrm{SEC})$

(DEG-TRUE)

$\begin{array}{llrr}1.2+-0.3 & -0.3+-0.2 & 19+-82 & 325+-31 \\ 0.7+-0.4 & -0.1+-0.4 & 101+-65 & 357+-56 \\ 0.5+-0.2 & -0.1+-0.1 & 318+-87 & 347+-15 \\ 0.3 & +0.1 & 294 & 344 \\ 0.5+-0.3 & +0.2+-0.2 & 101+-82 & 26+-9 \\ & & & \\ 2.6+-0.9 & -0.4+-0.3 & 311+-90 & 23+-59 \\ 1.5+-0.5 & -0.3+-0.5 & 94+-68 & 23+-56 \\ 1.0+-0.3 & -0.3+-0.3 & 12+-66 & 331+-56 \\ 0.8+-0.5 & +0.1+-0.4 & 58+-80 & 48+-29 \\ 1.6+-0.3 & +0.2+-0.4 & 342+-49 & 313+-42 \\ 0.8+-0.4 & -0.1+-0.1 & 28+-59 & 321+-34 \\ 0.7+-0.3 & -0.1+-0.2 & 136+-104 & 3+-70 \\ 0.6+-0.5 & -0.2+-0.3 & 36+-67 & 332+-52 \\ 1.1+-0.4 & -0.1+-0.4 & 297+-55 & 42+-40 \\ & & & \\ 2.3+-0.4 & -1.3+-0.4 & 62+-47 & 35+-59 \\ 1.9+-0.3 & -0.9+-0.7 & 84+-45 & 23+-39 \\ & & & \\ 1.8+-0.4 & -0.9+-0.6 & 89+-81 & 24+-65 \\ 1.5+-0.5 & -0.6+-0.6 & 86+-33 & 22+-36 \\ & & & \\ 1.1+-0.3 & -0.2+-0.4 & 69+-100 & 32+-73 \\ 0.7+-0.3 & +0.1+-0.1 & 327+-67 & 27+-28\end{array}$

NANTUCKET SHOALS

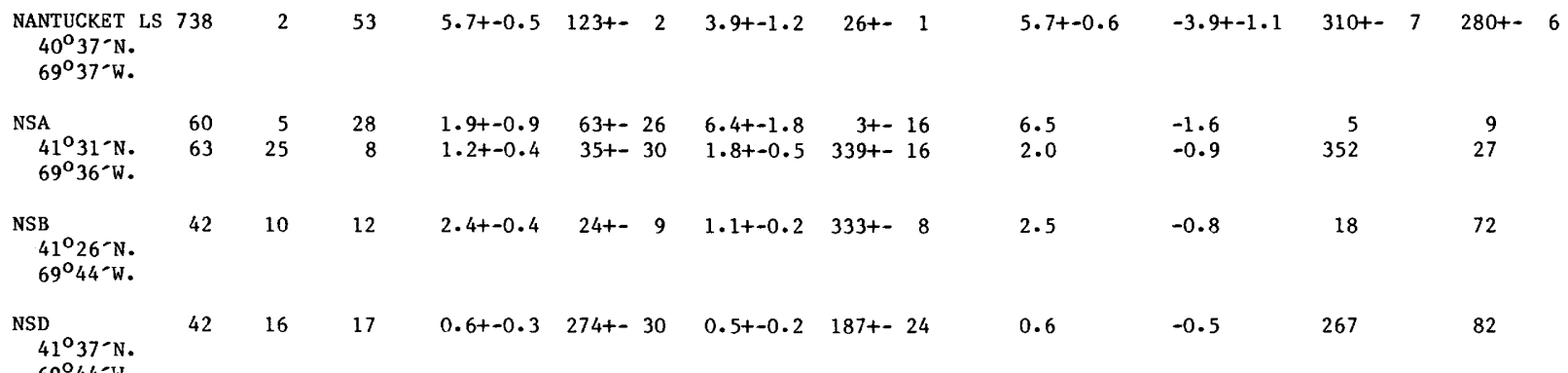

Instrument type, method of analysis and source of data are 1isted alphabetically by stations in Tables 3. If UMINOR < 0 , ellipse rotates clockwise

* - two separate moorings at different depths

** - tripod and subsurface mooring were at different depths 
Table 9. $\mathrm{O}_{1}$ tidal current parameters-Continued

Table 9. 01 TIDAL CURRENT PARAMETERS--Continued

\begin{tabular}{|c|c|c|c|c|c|c|c|c|c|c|c|}
\hline STATION & RECORD & INSTR & ABOVE & & OURIER & COEFFICIENTS & & & ENT ELLIPSE & PARAMETERS & \\
\hline $\begin{array}{l}\text { LAT. } \\
\text { LONG. }\end{array}$ & $\begin{array}{l}\text { LENGTH } \\
\text { (DAYS) }\end{array}$ & $\begin{array}{l}\text { DEPTH } \\
\text { (M) }\end{array}$ & $\begin{array}{l}\text { BOTTOM } \\
\text { (M) }\end{array}$ & $\begin{array}{c}\text { EAST } \\
\text { (CM/SEC) }\end{array}$ & $\begin{array}{l}\text { PHASE } \\
\text { (DEG-G) }\end{array}$ & $\begin{array}{c}\text { NORTH } \\
(\mathrm{CM} / \mathrm{SEC})\end{array}$ & $\begin{array}{r}\text { PHASE } \\
(D E G-G)\end{array}$ & $\begin{array}{r}\text { UMAJOR } \\
(\mathrm{CM} / \mathrm{SEC})\end{array}$ & $\begin{array}{c}\text { UMINOR } \\
(\mathrm{CM} / \mathrm{SEC})\end{array}$ & $\begin{array}{r}\text { PHASE } \\
(\text { DEG-G) }\end{array}$ & $\begin{array}{c}\text { ORIEN } \\
\text { (DEG-TRUE) }\end{array}$ \\
\hline
\end{tabular}

NANTUCKET SHOALS--CONT.

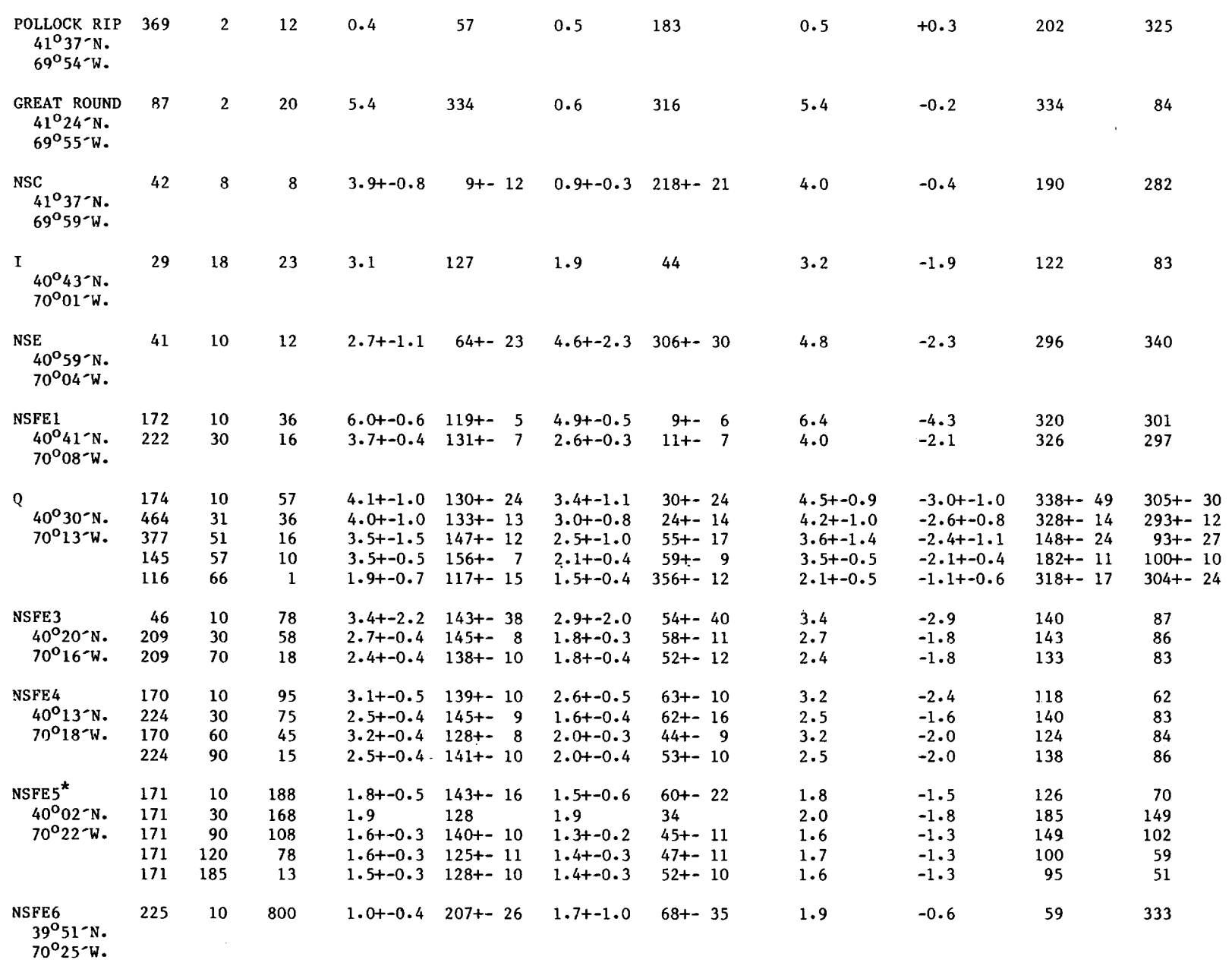

Instrument type, method of analysis and source of data are listed alphabetically by stations in Table 3 . If UMINOR $<0$, ellipse rotates clockwise

* Orientation is questionable 
Table 9. $\mathrm{O}_{1}$ tidal current parameters-Continued

Table 9. 01 TIDAL CURRENT PARAMETERS--Continued

$\begin{array}{cl}\text { STATION } & \text { RECORD INSTR ABOVE } \\ \text { LAT. } & \text { LENGTH DEPTH BOTTOM }\end{array}$

LONG. (DAYS) (M) (M)

$\begin{array}{cccr} & \text { FOURIER } & \text { COEFFICIENTS } & \\ \text { EAST } & \text { PHASE } & \text { NORTH } & \text { PHASE } \\ (\mathrm{CM} / \mathrm{SEC}) & (\mathrm{DEG}-\mathrm{G}) & (\mathrm{CM} / \mathrm{SEC}) & (\mathrm{DEG}-\mathrm{G})\end{array}$

CURRENT ELLIPSE
UMAJOR
$\begin{array}{cc}\text { UMINOR } \\ \text { UM/SEC }) & \text { (CM/SEC) }\end{array}$

PARAMETERS

PHASE ORIEN

(DEG-G) (DEG-TRUE)

NEW ENGLAND SHELF

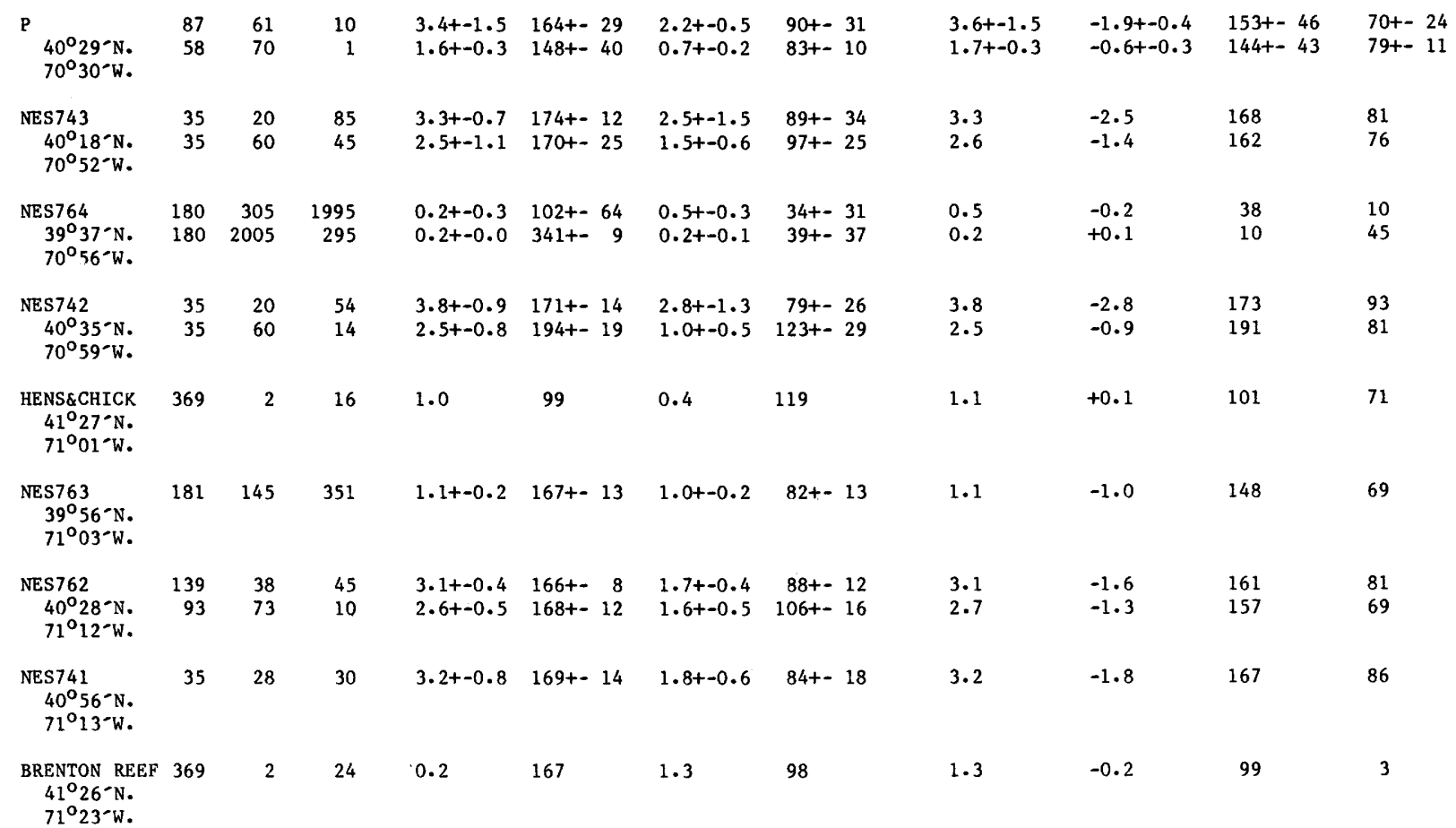

MIDDLE ATLANTIC BIGHT

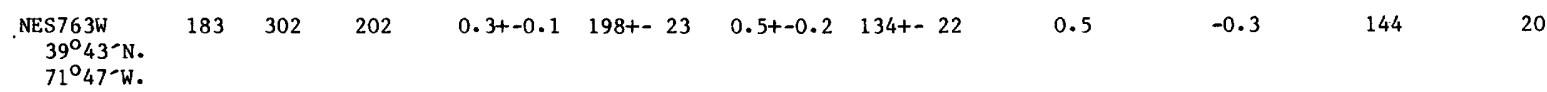
- - - - - - - -

Instrument type, method of analysis and source of data are 1isted alphabetically by stations in Table 3. If UMINOR $<0$, ellipse rotates clockwise 
Table 9. $\mathrm{O}_{1}$ tidal current parameters-Continued

Table 9. 01 TIDAL CURRENT PARAMETERS--Continued

\begin{tabular}{|c|c|c|c|c|c|c|c|c|c|c|}
\hline STATION & RECORD & INSTR & ABOVE & & OURIER & COEFFICIENTS & & & ENT ELLIPSE & PARAMETERS \\
\hline $\begin{array}{l}\text { LAT. } \\
\text { IONG. }\end{array}$ & $\begin{array}{l}\text { LENGTH } \\
\text { (DAYS) }\end{array}$ & $\begin{array}{l}\text { DEPTH } \\
\text { (M) }\end{array}$ & $\begin{array}{l}\text { BOTTOM } \\
\text { (M) }\end{array}$ & $\begin{array}{c}\text { EAST } \\
(\mathrm{CM} / \mathrm{SEC})\end{array}$ & $\begin{array}{l}\text { PHASE } \\
\text { (DEG-G) }\end{array}$ & $\begin{array}{c}\text { NORTH } \\
(\mathrm{CM} / \mathrm{SEC})\end{array}$ & $\begin{array}{r}\text { PHASE } \\
(D E G-G)\end{array}$ & $\begin{array}{r}\text { UMAJOR } \\
\text { (CM/SEC) }\end{array}$ & $\begin{array}{l}\text { UMINOR } \\
(\mathrm{CM} / \mathrm{SEC})\end{array}$ & $\begin{array}{c}\text { ORIEN } \\
\text { (DEG-TRUE) }\end{array}$ \\
\hline
\end{tabular}

MIDDLE ATLANTIC BIGHT--CONT.

\begin{tabular}{|c|c|c|c|c|c|c|c|c|c|c|c|c|}
\hline $\begin{array}{l}\text { NES762W } \\
39^{\circ} 55^{\circ} \mathrm{N} . \\
71^{\circ} 58^{\circ} \mathrm{W} .\end{array}$ & 172 & 38 & 45 & $3.1+-0.5$ & $212+-10$ & $2.7+-0.6$ & $143+-12$ & 3.4 & $-2 \cdot 3$ & 187 & & 56 \\
\hline $\begin{array}{l}\text { LT5 } \\
40^{\circ} 12^{\circ} \mathrm{N} . \\
72^{\circ} 00^{\circ} \mathrm{W} .\end{array}$ & $\begin{array}{r}87 \\
70 \\
116 \\
70 \\
87 \\
70 \\
87 \\
70\end{array}$ & $\begin{array}{l}21-H \\
21-R \\
41-H \\
41-R \\
61-H \\
61-R \\
66-H \\
66-R\end{array}$ & $\begin{array}{r}46 \\
46 \\
26 \\
26 \\
6 \\
6 \\
1 \\
1\end{array}$ & $\begin{array}{l}2.9+-1.7 \\
3.0 \\
3.8+-1.1 \\
3.5 \\
1.9+-0.4 \\
1.7 \\
1.4+-0.2 \\
1.2\end{array}$ & $\begin{array}{l}203+-16 \\
205 \\
193+-10 \\
192 \\
224+-24 \\
234 \\
232+-26 \\
248\end{array}$ & $\begin{array}{l}3.2+-0.8 \\
3.0 \\
3.1+-1.3 \\
3.1 \\
2.1+-0.0 \\
1.9 \\
1.6+-0.3 \\
1.6\end{array}$ & $\begin{array}{l}138+-11 \\
141 \\
129+-32 \\
132 \\
178+-22 \\
182 \\
183+-18 \\
192\end{array}$ & $\begin{array}{l}3.8+-1.5 \\
3.6 \\
4.4+-1.5 \\
4.1 \\
2.6+-0.2 \\
2.3 \\
1.9+-0.2 \\
1.8\end{array}$ & $\begin{array}{l}-2.1+-0.9 \\
-2.2 \\
-2.2+-0.8 \\
-2.3 \\
-1.1+-0.1 \\
-1.1 \\
-0.8+-0.2 \\
-0.9\end{array}$ & $\begin{array}{l}159+- \\
173 \\
165+- \\
168 \\
199+- \\
204 \\
202+- \\
209\end{array}$ & $\begin{array}{r}8 \\
99\end{array}$ & $\begin{array}{l}32+-23 \\
45 \\
34+-69 \\
52 \\
42+-8 \\
40 \\
38+-12 \\
31\end{array}$ \\
\hline $\begin{array}{l}\text { LT4 } \\
40^{\circ} 34^{-} \mathrm{N} . \\
72^{\circ} 19^{-} \mathrm{W} .\end{array}$ & $\begin{array}{r}29 \\
203 \\
174 \\
87\end{array}$ & $\begin{array}{r}3 \\
24 \\
44 \\
51\end{array}$ & $\begin{array}{r}49 \\
28 \\
8 \\
1\end{array}$ & $\begin{array}{l}1.8+-0.4 \\
5.4+-1.0 \\
3.4+-1.0 \\
1.3+-0.4\end{array}$ & $\begin{array}{lr}169+- & 49 \\
203+- & 21 \\
217+- & 15 \\
217+- & 6\end{array}$ & $\begin{array}{l}1.0+-1.1 \\
2.3+-1.2 \\
1.9+-0.2 \\
1.0+-0.1\end{array}$ & $\begin{array}{l}225+-112 \\
138+-37 \\
180+-38 \\
190+-2\end{array}$ & $\begin{array}{l}2.0+-0.6 \\
5.7+-1.2 \\
3.8+-0.7 \\
1.6+-0.4\end{array}$ & $\begin{array}{l}-0.6+-1.0 \\
-1.4+-0.9 \\
-0.9+-0.8 \\
-0.4+-0.0\end{array}$ & $\begin{array}{l}158+- \\
201+- \\
213+- \\
206+-\end{array}$ & $\begin{array}{r}33 \\
23 \\
21 \\
3\end{array}$ & $\begin{array}{lr}76+- & 33 \\
79+- & 16 \\
64+- & 14 \\
52+- & 4\end{array}$ \\
\hline $\begin{array}{l}\text { CMICE } \\
40^{\circ} 47^{\circ} \mathrm{N} . \\
72^{\circ} 29^{\circ} \mathrm{W} .\end{array}$ & $\begin{array}{l}25 \\
25 \\
25 \\
25\end{array}$ & $\begin{array}{r}4 \\
8 \\
16 \\
25\end{array}$ & $\begin{array}{r}25 \\
21 \\
13 \\
4\end{array}$ & $\begin{array}{l}3.5+-1.0 \\
3.3+-0.9 \\
3.3+-1.4 \\
2.1+-1.1\end{array}$ & $\begin{array}{l}176+-18 \\
175+-14 \\
167+-24 \\
168+-32\end{array}$ & $\begin{array}{l}0.3+-0.3 \\
0.3+-0.3 \\
1.1+-0.9 \\
1.0+-0.5\end{array}$ & $\begin{array}{r}277+-58 \\
318+-58 \\
12+-48 \\
262+-27\end{array}$ & $\begin{array}{l}3.5 \\
3.3 \\
3.4 \\
2.1\end{array}$ & $\begin{array}{l}+0.3 \\
+0.2 \\
-0.4 \\
+1.0\end{array}$ & $\begin{array}{l}176 \\
175 \\
169 \\
167\end{array}$ & & $\begin{array}{r}91 \\
94 \\
107 \\
93\end{array}$ \\
\hline $\begin{array}{l}\mathrm{ME} \\
39^{\circ} 57^{\circ} \mathrm{N} . \\
72^{\circ} 36^{-} \mathrm{W} .\end{array}$ & 29 & 59 & 1 & 2.4 & 213 & 1.1 & 178 & 2.6 & -0.6 & 208 & & 68 \\
\hline $\begin{array}{l}\mathrm{NJ} 4 \\
38^{\circ}{ }^{\circ} 5^{-\mathrm{N}} \text {. } \\
72^{\circ} 58^{-} \mathrm{W} .\end{array}$ & $\begin{array}{l}72 \\
72 \\
72\end{array}$ & $\begin{array}{r}3 \\
43 \\
91\end{array}$ & $\begin{array}{r}89 \\
49 \\
1\end{array}$ & $\begin{array}{l}3.6+-2.3 \\
1.4+-0.5 \\
0.1+-0.0\end{array}$ & $\begin{array}{l}325+-37 \\
289+-21 \\
346+-11\end{array}$ & $\begin{array}{l}3.0+-1.4 \\
1.0+-0.3 \\
0.1+-0.0\end{array}$ & $\begin{array}{l}255+-26 \\
243+-18 \\
132+-10\end{array}$ & $\begin{array}{l}3.9 \\
1.6 \\
0.1\end{array}$ & $\begin{array}{l}-2.6 \\
-0.6 \\
+0.0\end{array}$ & $\begin{array}{l}303 \\
275 \\
329\end{array}$ & & $\begin{array}{r}59 \\
58 \\
135\end{array}$ \\
\hline $\begin{array}{l}\mathrm{MA} \\
\quad 39^{\circ} 27^{\circ} \mathrm{N} . \\
73^{\circ} 00^{-} \mathrm{W} .\end{array}$ & 58 & 58 & 1 & $0.9+-0.0$ & $263+-28$ & $0.8+-0.0$ & $204+-33$ & $1.0+-0.1$ & $-0.6+-0.0$ & $240+-$ & 28 & $53+-$ \\
\hline $\begin{array}{l}\mathrm{LT} 3 \\
39^{\circ} 16^{-} \mathrm{N} . \\
73^{\circ} 02^{-} \mathrm{W} .\end{array}$ & $\begin{array}{r}104 \\
104 \\
70\end{array}$ & $\begin{array}{r}9 \\
19 \\
58\end{array}$ & $\begin{array}{l}61 \\
51 \\
12\end{array}$ & $\begin{array}{l}1.2+-0.6 \\
2.2+-1.3 \\
1.4+-0.5\end{array}$ & $\begin{array}{r}282+-30 \\
279+-34 \\
4+-22\end{array}$ & $\begin{array}{l}1.5+-0.5 \\
1.4+-0.4 \\
1.8+-0.3\end{array}$ & $\begin{array}{l}217+-18 \\
206+-18 \\
274 t-10\end{array}$ & $\begin{array}{l}1.6 \\
2.3 \\
1.8\end{array}$ & $\begin{array}{l}-1.0 \\
-1.3 \\
-1.4\end{array}$ & $\begin{array}{l}237 \\
270 \\
274\end{array}$ & & $\begin{array}{r}31 \\
74 \\
0\end{array}$ \\
\hline $\begin{array}{l}\text { MESA7 } \\
39^{\circ} 55^{\circ} \mathrm{N} . \\
73^{\circ} 06^{\circ} \mathrm{W} .\end{array}$ & $\begin{array}{r}107 \\
103 \\
63\end{array}$ & $\begin{array}{l}18 \\
38 \\
66\end{array}$ & $\begin{array}{r}50 a \\
30 a \\
2 a\end{array}$ & $\begin{array}{l}2.0+-1.6 \\
1.3+-0.7 \\
0.5+-0.3\end{array}$ & $\begin{array}{l}243+-45 \\
233+-30 \\
226+-30\end{array}$ & $\begin{array}{l}2.8+-0.9 \\
1.0+-0.5 \\
0.4+-0.1\end{array}$ & $\begin{array}{l}177+-18 \\
206+-26 \\
301+-10\end{array}$ & $\begin{array}{l}3.0 \\
1.6 \\
0.5\end{array}$ & $\begin{array}{l}-1.7 \\
-0.4 \\
+0.4\end{array}$ & $\begin{array}{l}192 \\
223 \\
244\end{array}$ & & $\begin{array}{l}25 \\
53 \\
66\end{array}$ \\
\hline FIRE IS. & 369 & 2 & 27 & 1.3 & 220 & 0.8 & 187 & 1.5 & -0.4 & 211 & & 59 \\
\hline
\end{tabular}
$40^{\circ} 29^{-} \mathrm{N}$. $73^{\circ} 11^{-} \mathrm{W}$.

Instrument type, method of analysis and source of data are listed alphabetically by stations in Table 3 .

If $U$ IMINOR $<0$, ellipse rotates clockwise

$H$ - harmonic analysis $R$ - response analysis

a - water depth taken from chart 13200 
Table 9. $\mathrm{O}_{1}$ tidal current parameters-Continued

Table 9. 01 TIDAL CURRENT PARAMETERS--Continued

$\begin{array}{cccc} & \text { FOURIER } & \text { COEFFICIENTS } & \\ \text { EAST } & \text { PHASE } & \text { NORTH } & \text { PHASE } \\ (\mathrm{CM} / \mathrm{SEC}) & (\mathrm{DEG}-\mathrm{G}) & (\mathrm{CM} / \mathrm{SEC}) & (\mathrm{DEG}-\mathrm{G})\end{array}$

\begin{tabular}{cccc}
\multicolumn{2}{c}{ CURRENT ELLIPSE } & PARAMETERS \\
UMAJOR & UMINOR & PHASE & ORIEN \\
$(\mathrm{CM} / \mathrm{SEC})$ & $(\mathrm{CM} / \mathrm{SEC})$ & $(\mathrm{DEG}-\mathrm{G})$ & $($ DEG-TRUE)
\end{tabular}

$\begin{array}{cl}\text { STATION } & \text { RECORD INSTR ABOVE } \\ \text { LAT. } & \text { LENGTH DEPTH BOTTOM }\end{array}$

LONG. (DAYS) (M) (M)

MIDDLE ATLANTIC BIGHT--CONT.

$\begin{array}{llr}\text { MF } & 116 & 15 \\ 38^{\circ} 31-\mathrm{N} . & 116 & 232\end{array}$ $73^{\circ} 14^{-\mathrm{W}}$.

MC $38^{\circ} 33-\mathrm{N}$.

$29 \quad 79 \quad 1$

$73^{\circ} 31^{\top} \mathrm{W}$.

MB $38^{\circ} 44^{-}$ $73^{\circ} 38^{-\mathrm{W}}$.

$\begin{array}{rr}406 & 15 \\ 58 & 45 \\ 522 & 50 \\ 58 & 54 \\ 87 & 59\end{array}$

LT2 $39^{\circ} 24-\mathrm{N} .116-\mathrm{C}$ $73^{\circ} 43-\mathrm{W}$.

BARNEGAT $39^{\circ} 46^{-\mathrm{N}}$.

$73^{\circ} 56^{-} \mathrm{W}$.

MD

$74^{\circ} \mathrm{O} 2-\mathrm{W}$.

$\begin{array}{lrr}\text { L. EGG INLET } & 264 & 5 \\ 39^{\circ} 28^{\prime} \mathrm{N} . & 365 & 10 \\ 74^{\circ} 15^{\circ} \mathrm{W} . & \end{array}$

NE END L.S. $369 \quad 2 \quad 34 \quad 2.1$ $38^{\circ} 58^{-\mathrm{N}}$. $74^{\circ} 30^{-} \mathrm{W}$.

0.5 $38^{\circ} 59^{-N} \mathrm{~N}$

SOUTHERN MID-ATI.ANTIC BIGHT

$37^{\circ} 55^{\circ} \mathrm{N}$.

$74^{\circ} 56^{-\mathrm{W}}$.

MAB

$\begin{array}{llr}A \mathrm{AB}^{\circ} & 53 & 9 \\ 36^{\circ} 50^{-\mathrm{N}} . & 52 & 21 \\ 75^{\circ} \mathrm{O} 2^{-\mathrm{W}} & 53 & 32\end{array}$

$75^{\circ} \mathrm{O} 2^{-\mathrm{W}}$.

DIAMOND $35^{\circ} 05^{-\mathrm{N}}$.

$75^{\circ} 20^{-} \mathrm{W}$.

CHESAPEAKE

$36^{\circ} 59^{-N}$. $75^{\circ} 42^{-} \mathrm{W}$.

Instrument type, method of analysis and source of data are listed alphabetically by stations in Table 3 .

If UMINOR < 0 , ellipse rotates clockwise

c - average of 4 records $31,31,32$, and 29 meters above bottom
$1.2+-1.2 \quad 286+-30 \quad 1.8+-1.2 \quad 234+-86$

$0.3+-0.1 \quad 23+-96 \quad 0.3+-0.1 \quad 238+-88$

53

1.0

304

$2.3+-0.8 \quad 312+-42$

$\begin{array}{lll}3.7+-0.1 & 272 t-33\end{array}$

$1.4+-0.5326+-32$

$1.1+-0.2 \quad 10+-1$

$0.7+-0.2 \quad 278+-92$

$1.5+-0.7 \quad 264+-30$

$1.7+-0.1300+-11$

$0.7+-0.3 \quad 231+-44$

$2.7+-0.9266+-46$

$2.2+-0.9220+-28$

4. $0+-1.4 \quad 268+-25 \quad 3.6+-0.8 \quad 235+-20$

0.5263

0.9

233

$0.4 t-0.4 \quad 301+-96$

$1.0+-0.4$

$299+-26$

2.7
2.4

57

68

$1 \cdot 1$

294

63

$\begin{array}{lllr}1.9+-1.1 & -0.8+-1.6 & 226+-88 & 102+-27 \\ 0.4+-0.1 & -0.1+-0.1 & 259+-101 & 32+-51 \\ 1.0 & -0.5 & 299 & 348\end{array}$

$2.9+-0.9 \quad-1.1+-0.7 \quad 284+-50 \quad 49+-72$

$2.1+-0.3 \quad-1.2+-0.4 \quad 298+-35 \quad 40+-17$

$1.8+-0.7 \quad-0.9+-0.4 \quad 288+-31 \quad 38+-74$

$1.8+-0.1 \quad-1.0+-0.3 \quad 310+-8 \quad 2+-0$

$0.9+-0.2 \quad-0.2+-0.6 \quad 194+-28 \quad 340+-59$

$3.1+-1.1 \quad-1.3+-0.8 \quad 249+-39 \quad 55+-3$

$\begin{array}{llll}5.2+-1.4 & -1.5+-0.7 & 253+-26 & 47+-\end{array}$

$\begin{array}{llll}1.0 & -0.2 & 239 & 26\end{array}$

$1.1+-0.4 \quad-0.0+-0.0 \quad 298+-26 \quad 7+-30$

$\begin{array}{llll}3.6 & -0.7 & 67 & 43\end{array}$

$\begin{array}{llll}2.8 & -0.1 & 64 & 30\end{array}$

$\begin{array}{llll}2.3 & +0.2 & 283 & 63\end{array}$

.2

1.2

$-1 \cdot 2$

97

38

$1.6+-0.8 \quad 270+-29 \quad 3.7+-1.8 \quad 134+-28$

$4.0+-2.6 \quad 218+-38 \quad 3.6+-2.1 \quad 135+-34$

3.9

4.1

$-1 \cdot 1$

-3.5
+0.2

129

197

341

$2 \cdot 1+-0.0$

$-0.9+-0.6$

$299+-64$

288

$1.4+-0.7 \quad 324+-57 \quad 1.8+-0.3 \quad 273+-34$

340

1.1

140

1.3

$+0.2$

145

332 
Table 9. $\mathrm{O}_{1}$ tidal current parameters-Continued

Table 9. 01 TIDAL CURRENT PARAMETERS-Continued

\begin{tabular}{|c|c|c|c|c|c|c|c|c|c|c|c|}
\hline STATION & RECORD & INSTR & ABOVE & & JURIER & COEFFICIENTS & & CUR & ENT ELLIPSE & PARAMETERS & \\
\hline $\begin{array}{l}\text { LAT. } \\
\text { LONG. }\end{array}$ & $\begin{array}{l}\text { LENGTH } \\
\text { (DAYS) }\end{array}$ & $\begin{array}{l}\text { DEPTH } \\
\text { (M) }\end{array}$ & $\begin{array}{l}\text { BОTTOM } \\
\text { (M) }\end{array}$ & $\begin{array}{c}\text { EAST } \\
(\mathrm{CM} / \mathrm{SEC})\end{array}$ & $\begin{array}{c}\text { PHASE } \\
\text { (DEG-G) }\end{array}$ & $\begin{array}{c}\text { NORTH } \\
(\mathrm{CM} / \mathrm{SEC})\end{array}$ & $\begin{array}{r}\text { PHASE } \\
(\text { DEG-G) }\end{array}$ & $\begin{array}{r}\text { UMAJOR } \\
\text { (CM/SEC) }\end{array}$ & $\begin{array}{l}\text { UMINOR } \\
\text { (CM/SEC) }\end{array}$ & $\begin{array}{c}\text { PHASE } \\
\text { (DEG-G) }\end{array}$ & $\begin{array}{c}\text { ORIEN } \\
\text { (DEG-TRUE) }\end{array}$ \\
\hline
\end{tabular}

OCEANIC

$\begin{array}{lrrr}\mathrm{S} 8 & 415 & 70 & 2470 \\ 42^{\circ} 00^{-} \mathrm{N} . & 415 & 1500 & 1040\end{array}$

$0.5+-0.4 \quad 276+-105 \quad 0.5+-0.4 \quad 117+-4$

$0.1+-0.1 \quad 350+-5 \quad 0.3+-0.1 \quad 179+-6$

$63^{\circ} 30^{-} \mathrm{W} . \quad 96 \quad 2530 \quad 10$

0.1

0.2

150

$0.6+-0.4 \quad-0.3+-0.6 \quad 144+-24 \quad 9+-51$

$0.3+-0.1 \quad-0.0+-0.0 \quad 176+-5 \quad 330+-5$

NES 765

$180 \quad 1995 \quad 655$

$0.2+-0.1 \quad 328+-12 \quad 0.1+-0.0 \quad 91+-16$

0.2

$\begin{array}{lll}-0.0 & 151 & 334\end{array}$

$39^{\circ} 17^{-N}$.

$70^{\circ} 50^{\circ} \mathrm{W}$.

Instrument type, method of analysis and source of data are 1isted alphabetically by stations in Table 3 .

If UMINOR < 0, ellipse rotates clockwise

c - average of 4 records C $31,31,32$, and 29 meters above bottom 

APPENDIXES I-III 



\section{APPENDIX I - INSTRUMENT DESCRIPTION}

This appendix is a brief description of the instruments used to make the current or pressure observations listed in this atlas. Use of brand names in this atlas is for descriptive purposes only and does not constitute endorsement by the U.S. Geological Survey.

Aanderaa Model RCM-4 current meter. Speed sensor is a 6-cup Savonius rotor. Instrument is oriented into the current by a large trailing vane. Rotor count and a single measurement of direction are recorded every 10-20 minutes (Beardsley and others, 1977a).

Braincon Model 316 or 381 current meter. Data recorded on film.

Bubbler Sea level is determined by the back pressure at an oriface to which nitrogen gas or compressed air is supplied. Data is recorded by a Bristol strip chart recorder. Accuracy is $\pm 7.6 \mathrm{~cm}$ and \pm 5 minutes as cited in EG\&G (1976). (Redfield, 1962).

C\&GS Sea level is measured by a float in a stilling well. Accuracy is estimated at $\pm 5.8 \mathrm{~cm}$ and 12 minutes (Goodrich, 1981).

Current pole Current speed is measured by the rate of drift of a $4.6 \mathrm{~m}$ wooden pole which floats with $4.3 \mathrm{~m}$ below the sea surface. Current direction is determined with a pelorus and compass (Haight, 1941).

Draper Pressure is measured by means of a bonded strain gauge pressure transducer. Accuracy is estimated at $\pm 3-4 \mathrm{~cm}$ (Beardsley and others, 1977b).

EG\&G Model 850 current meter. Speed sensor is a Savonius rotor and the direction sensor is a small current vane. Both sensors are burst-sampled at a $5 \mathrm{~s}$ rate, typically 10 times every 7.5 minutes. (Beardsley and others, 1977a).

Model 102 current meter. Approximately the same instrument as the Model 850 , but data is recorded on film.

Endeco Model 105 current meter. Speed sensor is an 8-bladed bi-directional ducted impeller which is oriented into the current by a large leading vane. Direction is recorded on $16 \mathrm{~mm}$ film as a time exposure over a measurement interval which is typically 30 minutes. (Beardsley and others, 1977a).

Filloux Tide pressure is measured by means of a Bourdon tube-optical lever transducer. Accuracy is estimated at $\pm 0.5 \mathrm{~cm}$. (Beardsley and others, 1977b).

Fisher-Porter Sea level is measured by a float in a stilling well. Accuracy is $\pm 5.8 \mathrm{~cm}$ and 6 minutes (Goodrich, 1981).

Hewlett-Packard Pressure is measured by means of a quartz crystal. Sampling interval is 2 minutes and precision is about 0.1 millibar.

Ottboro Pressure gauge transducers connected to bellows which control a pen on a clock-driven strip chart. Accuracy is estimated to be about 3 percent (Canadian Hydrographic Service, 1966).

Paroscientific Pressure is measured by a quartz-crystal sensor. Accuracy is estimated at \pm 3.0 millibars (Butman and Folger, 1979).

Photographic Current speed sensor is a paddle-wheel impeller. Counter and compass heading are recorded photographically (Canadian Hydrographic Service, 1966).

RAY Marsh-McBirney two-axis electromagnetic current sensor mounted on a buoy system fabricated by Raytheon Ocean Systems (Lobecker and others, 1978).

USGS tripod Current speed is measured by a Savonius rotor and current direction by a small vane. Sensors are burst sampled at a $4 \mathrm{~s}$ rate typically 12 times every 7.5 minutes. Accuracy is estimated at $\pm 1 \mathrm{~cm} / \mathrm{s}$ and $\pm 5^{\circ}$ (Butman and Folger, 1979).

VACM AMF (now EG\&G) Model 610 vector averaging current meter. The instrument uses a Savonius rotor speed sensor and a small vane direction sensor. The sensors are sampled continuously and vector-averaged east and north current components are typically recorded every 7.5 or 15.0 minutes.

Vibratron Pressure gauge with taut vibrating wire sensor. Accuracy estimated at $\pm 0.5 \mathrm{~cm}$ (Beardsley and others, 1977b and Petrillo, 1981). 


\section{APPENDIX II - $\mathrm{M}_{2}$ BOTTOM CURRENT ESTIMATES}

Estimates of the amplitude of the major and minor axis of the $M_{2}$ bottom current (table II-1) were determined using the empirical curve in figure 2 and tidal constants at a point within about $30 \mathrm{~m}$ of the bottom. The phase and orientation were estimated by adding $-17^{\circ}$ and $+14^{\circ}$, respectively, when the measurements were made at depths greater than 15 mab. If the measurements were made at depths less than 15 mab then $-8^{\circ}$ and $+7^{\circ}$ were added to the corresponding phase and orientation to give values at $1 \mathrm{mab}$. The asterisk after a station name indicates actual field measurements at 1 mab and not estimates of bottom current.

Table II-1. $M_{2}$ bottom current

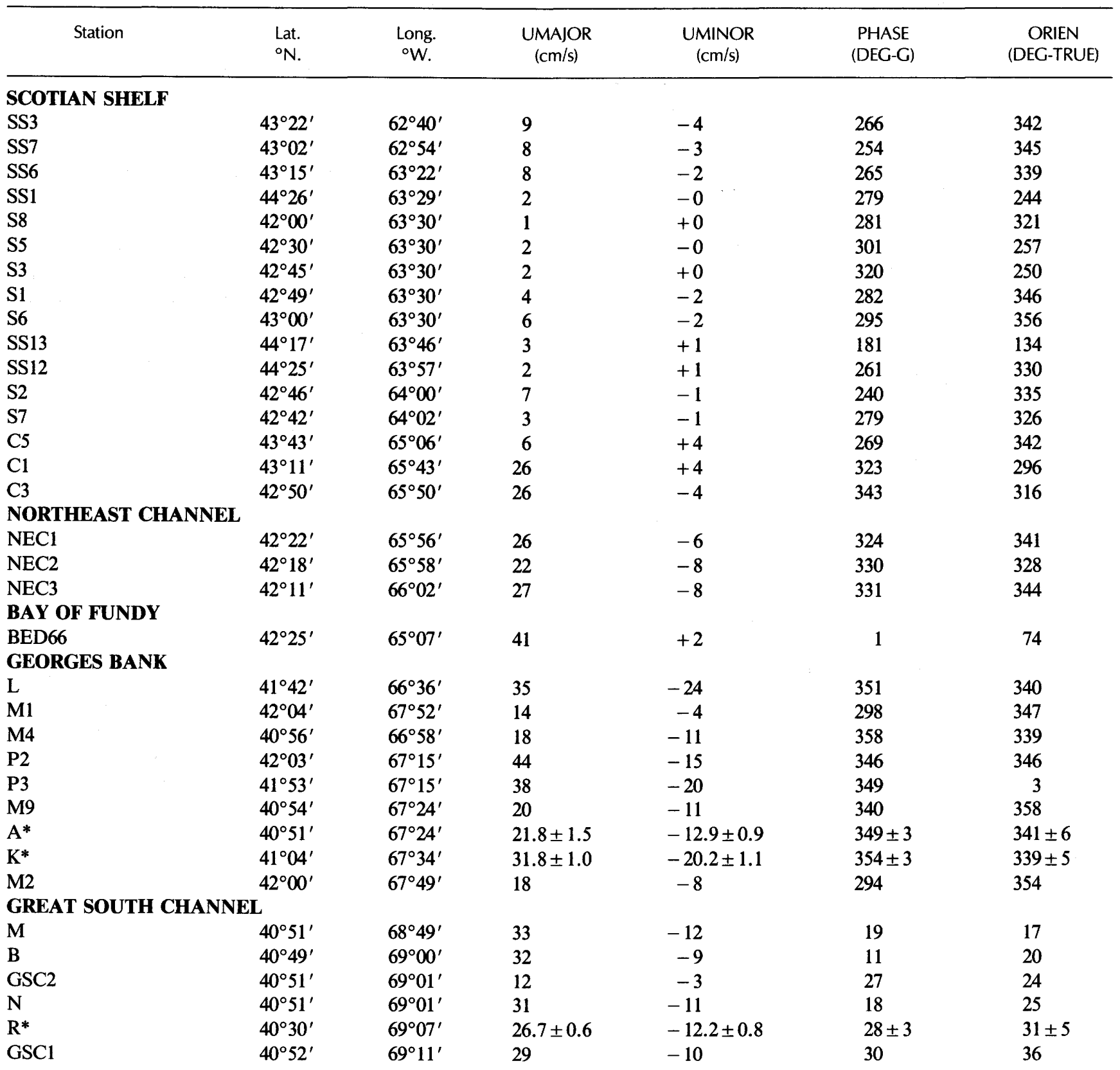


Table II-1. $\mathrm{M}_{2}$ bottom current-Continued

\begin{tabular}{|c|c|c|c|c|c|c|}
\hline Station & $\begin{array}{l}\text { Lat. } \\
{ }^{\circ} \mathrm{N} .\end{array}$ & $\begin{array}{l}\text { Long. } \\
{ }^{\circ} \mathrm{W} .\end{array}$ & $\begin{array}{l}\text { UMAJOR } \\
(\mathrm{cm} / \mathrm{s})\end{array}$ & $\begin{array}{l}\text { UMINOR } \\
(\mathrm{cm} / \mathrm{s})\end{array}$ & $\begin{array}{l}\text { PHASE } \\
\text { (DEG-G) }\end{array}$ & $\begin{array}{c}\text { ORIEN } \\
\text { (DEG-TRUE) }\end{array}$ \\
\hline \multicolumn{7}{|c|}{ LYDONIA CANYON } \\
\hline LCA* & $40^{\circ} 34^{\prime}$ & $67^{\circ} 45^{\prime}$ & $17.6 \pm 1.5$ & $-8.3 \pm 3.1$ & $346 \pm 0$ & $337 \pm 2$ \\
\hline LCB & $40^{\circ} 32^{\prime}$ & $67^{\circ} 43^{\prime}$ & 4 & +1 & $\sim 145$ & 344 \\
\hline LCE & $40^{\circ} 25^{\prime}$ & $67^{\circ} 40^{\prime}$ & 11 & +0 & 351 & 38 \\
\hline $\mathrm{LCH}$ & $40^{\circ} 18^{\prime}$ & $67^{\circ} 40^{\prime}$ & 4 & -1 & 326 & 24 \\
\hline LCI & $40^{\circ} 23^{\prime}$ & $67^{\circ} 33^{\prime}$ & 4 & -2 & $\sim 300$ & $\sim 340$ \\
\hline $\mathbf{L C L}$ & $40^{\circ} 32^{\prime}$ & $67^{\circ} 36^{\prime}$ & 13 & -9 & 349 & 354 \\
\hline LCM $^{*}$ & $40^{\circ} 30^{\prime}$ & $67^{\circ} 49^{\prime}$ & $15.5 \pm 3.6$ & $-9.9 \pm 2.4$ & $333 \pm 11$ & $344 \pm 11$ \\
\hline \multicolumn{7}{|c|}{ NANTUCKET SHOALS } \\
\hline NSA & $41^{\circ} 31^{\prime}$ & $69^{\circ} 36^{\prime}$ & 36 & -3 & 315 & 18 \\
\hline NSB & $41^{\circ} 26^{\prime}$ & $69^{\circ} 44^{\prime}$ & 40 & -11 & 336 & 42 \\
\hline NSD & $41^{\circ} 37^{\prime}$ & $69^{\circ} 44^{\prime}$ & 25 & +3 & 324 & 41 \\
\hline POLLOCK RIP & $41^{\circ} 37^{\prime}$ & $69^{\circ} 54^{\prime}$ & 39 & -3 & 291 & 29 \\
\hline GREAT ROUND & $41^{\circ} 24^{\prime}$ & $69^{\circ} 55^{\prime}$ & 37 & -11 & 347 & 80 \\
\hline NSC & $41^{\circ} 37^{\prime}$ & $69^{\circ} 59^{\prime}$ & 29 & -5 & 18 & 119 \\
\hline NSE & $40^{\circ} 59^{\prime}$ & $70^{\circ} 04^{\prime}$ & 24 & -18 & 18 & 135 \\
\hline NSFE1 & $40^{\circ} 41^{\prime}$ & $70^{\circ} 08^{\prime}$ & 14 & -12 & 33 & 86 \\
\hline $\mathrm{Q}^{*}$ & $40^{\circ} 30^{\prime}$ & $70^{\circ} 13^{\prime}$ & $10.0 \pm 0.4$ & $-7.4 \pm 0.3$ & $41 \pm 2$ & $88 \pm 4$ \\
\hline NSFE3 & $40^{\circ} 20^{\prime}$ & $70^{\circ} 16^{\prime}$ & 7 & -5 & 36 & 66 \\
\hline NSFE4 & $40^{\circ} 13^{\prime}$ & $70^{\circ} 18^{\prime}$ & 5 & -4 & 37 & 59 \\
\hline NSFE5 & $40^{\circ} 02^{\prime}$ & $70^{\circ} 22^{\prime}$ & 3 & -2 & 38 & 85 \\
\hline \multicolumn{7}{|c|}{ NEW ENGLAND SHELF } \\
\hline $\mathrm{P}^{*}$ & $40^{\circ} 29^{\prime}$ & $70^{\circ} 30^{\prime}$ & $6.1 \pm 0.1$ & $-5.1 \pm 0.2$ & $16 \pm 11$ & $74 \pm 5$ \\
\hline NES742 & $40^{\circ} 35^{\prime}$ & $70^{\circ} 59^{\prime}$ & 5 & -4 & 24 & 76 \\
\hline HENS\&CHICK & $41^{\circ} 27^{\prime}$ & $71^{\circ} 01^{\prime}$ & 12 & -5 & 296 & 91 \\
\hline NES762 & $40^{\circ} 28^{\prime}$ & $71^{\circ} 12^{\prime}$ & 4 & -3 & 74 & 134 \\
\hline \multicolumn{7}{|c|}{ MIDDLE ATLANTIC BIGHT } \\
\hline P32 & $40^{\circ} 15^{\prime}$ & $71^{\circ} 51^{\prime}$ & 3 & -2 & 213 & 318 \\
\hline LT5* & $40^{\circ} 12^{\prime}$ & $72^{\circ} 00^{\prime}$ & $4.6 \pm 0.3$ & $-2.3 \pm 0.1$ & $226 \pm 18$ & $306 \pm 10$ \\
\hline P31 & $40^{\circ} 39^{\prime}$ & $72^{\circ} 15^{\prime}$ & 5 & -1 & 215 & 285 \\
\hline LT4* & $40^{\circ} 34^{\prime}$ & $72^{\circ} 19^{\prime}$ & $5.0 \pm 0.3$ & $-1.1 \pm 0.1$ & $232 \pm 5$ & $276 \pm 1$ \\
\hline CMICE & $40^{\circ} 47^{\prime}$ & $72^{\circ} 29^{\prime}$ & 5 & +0 & $\sim 219$ & 292 \\
\hline $\mathrm{ME}^{*}$ & $39^{\circ} 57^{\prime}$ & $72^{\circ} 36^{\prime}$ & 7.1 & -2.9 & 235 & 326 \\
\hline LTM & $40^{\circ} 07^{\prime}$ & $72^{\circ} 55^{\prime}$ & 7 & +3 & 262 & 315 \\
\hline NJ4* & $38^{\circ} 55^{\prime}$ & $72^{\circ} 58^{\prime}$ & 1 & +0 & 229 & 244 \\
\hline MA* $^{*}$ & $39^{\circ} 27^{\prime}$ & $73^{\circ} 00^{\prime}$ & $7.3 \pm 1.3$ & $-3.4 \pm 0.1$ & $226 \pm 9$ & $319 \pm 90$ \\
\hline LT3 & $39^{\circ} 16^{\prime}$ & $73^{\circ} 02^{\prime}$ & 7 & -4 & 255 & 320 \\
\hline LT7 & $39^{\circ} 55^{\prime}$ & $73^{\circ} 05^{\prime}$ & 6 & -2 & 264 & 320 \\
\hline MESA7 & $39^{\circ} 55^{\prime}$ & $73^{\circ} 06^{\prime}$ & 5 & -1 & 246 & 309 \\
\hline P12 & $39^{\circ} 09^{\prime}$ & $73^{\circ} 13^{\prime}$ & 6 & -4 & 250 & 318 \\
\hline MF & $38^{\circ} 31^{\prime}$ & $73^{\circ} 14^{\prime}$ & 2 & -1 & 190 & 320 \\
\hline NJ3A & $39^{\circ} 04^{\prime}$ & $73^{\circ} 20^{\prime}$ & 7 & -4 & 262 & 319 \\
\hline 15 & $40^{\circ} 26^{\prime}$ & $73^{\circ} 28^{\prime}$ & 7 & -1 & 250 & 302 \\
\hline $\mathrm{MC}^{*}$ & $38^{\circ} 33^{\prime}$ & $73^{\circ} 31^{\prime}$ & 6.7 & -3.1 & 250 & 321 \\
\hline 49 & $39^{\circ} 38^{\prime}$ & $73^{\circ} 34^{\prime}$ & 8 & -4 & 245 & 319 \\
\hline MB* & $38^{\circ} 44^{\prime}$ & $73^{\circ} 38^{\prime}$ & $7.3 \pm 1.3$ & $-3.4 \pm 0.4$ & $259 \pm 4$ & $316 \pm 2$ \\
\hline LT6 & $40^{\circ} 08^{\prime}$ & $73^{\circ} 38^{\prime}$ & 4 & +1 & 230 & 318 \\
\hline LT2 & $39^{\circ} 24^{\prime}$ & $73^{\circ} 43^{\prime}$ & 8 & -4 & 260 & 325 \\
\hline P11 & $39^{\circ} 17^{\prime}$ & $73^{\circ} 55^{\prime}$ & 7 & -3 & 245 & 318 \\
\hline MD* & $38^{\circ} 59^{\prime}$ & $74^{\circ} 02^{\prime}$ & $6.8 \pm 0.6$ & $-2.2 \pm 1.1$ & $244 \pm 15$ & $313 \pm 6$ \\
\hline L.EGG INLET & $39^{\circ} 28^{\prime}$ & $74^{\circ} 15^{\prime}$ & 4 & -1 & $\sim 275$ & $\sim 310$ \\
\hline \multicolumn{7}{|c|}{ SOUTHERN MID-ATLANTIC BIGHT } \\
\hline MAB & $36^{\circ} 50^{\prime}$ & $75^{\circ} 02^{\prime}$ & 7 & -3 & 273 & 251 \\
\hline CHESAPEAKE & $36^{\circ} 59^{\prime}$ & $75^{\circ} 42^{\prime}$ & 4 & -0 & 337 & 291 \\
\hline
\end{tabular}




\section{APPENDIX III - TIDE GENERATION}

The total current, $U$, or sea-level elevation, $H$, is represented by the sum of all the tidal constituents:

$$
\mathrm{U} \text { or } \mathrm{H}=\Sigma f \mathrm{u}_{\mathrm{i}} \cos \left(\omega_{\mathrm{i}} \mathrm{t}-\phi_{\mathrm{i}}\right) \quad \text { eq. III-1 }
$$

where $\mathrm{i}$ denotes a constituent, $\mathbf{u}_{\mathrm{i}}$ is the amplitude of either the east or north component of current or the amplitude of the sea-level elevation and $t$ is local time. A small correction due to the moon's node is given by " $\mathrm{f}$ " (see table III-1), and $\omega_{\mathrm{i}}$ is the frequency of the constituent in degrees per solar hour.

Table III-1. Correction, f, for moon's node (period $=18.61$ years) adapted from table 14 of Schureman (1941) for 1976-1980

\begin{tabular}{lccccc}
\hline Constituent & 1976 & 1977 & 1978 & 1979 & 1980 \\
\hline $\mathrm{M}_{2} \& \mathrm{~N}_{2}$ & 1.029 & 1.035 & 1.038 & 1.036 & 1.030 \\
$\mathrm{~K}_{1}$ & 0.916 & 0.891 & 0.882 & 0.890 & 0.913 \\
$\mathrm{O}_{1}$ & 0.863 & 0.822 & 0.806 & 0.819 & 0.858 \\
\hline
\end{tabular}

The phase lag, $\phi_{\mathrm{i}}$ of each constituent is measured from the 0 hour or the beginning of the desired time series, and it is related to the Greenwich phase $(G)$ and to $\mathrm{S}$, the longitude of the time meridian of observation, by equation 321 in Schureman (1941) which is

Greenwich phase $(\mathrm{G})=$ Greenwich $\left(\mathrm{V}_{\mathrm{o}}+\mathrm{u}\right)+\omega_{\mathrm{i}} \mathrm{S} / 15+\phi_{\mathrm{i}}$

thus

$$
\begin{aligned}
& \phi_{\mathrm{i}}= \text { Greenwich phase }(\mathrm{G})- \\
& \text { Greenwich }\left(\mathrm{V}_{\mathrm{o}}+\mathrm{u}\right)-\omega_{\mathrm{i}} \mathrm{S} / 15 \quad \text { eq. III-2 }
\end{aligned}
$$

where the Greenwich phase (G) can be found in this atlas. The Greenwich $\left(\mathrm{V}_{\mathrm{o}}+\mathrm{u}\right)$ is the time difference (expressed in degrees) between the transit of the Greenwich meridian of a fictitious moon (associated with each constituent and having a frequency $\omega_{\mathrm{i}}$ ) and the Greenwich 0 hour of the day the time series begins. Greenwich $\left(V_{o}+u\right)$ is found using tables 15, 16, 17, and 18 in Schureman (1941).

The three semidiurnal constituents $\mathrm{M}_{2}, \mathrm{~S}_{2}$, and $\mathrm{N}_{2}$ along with the two diurnal constituents $K_{1}$ and $O_{1}$ are the most important in generating a tidal series and these tidal frequencies and periods are listed in table 1.

Example: What are the equations for the east and north components of the tidal current at $60 \mathrm{~m}$ and the sea-level elevation at station $\mathrm{K}$ beginning February 1, 1978 at 0100 local time using the five major constituents $M_{2}, S_{2}, N_{2}, K_{1}$, and $\mathrm{O}_{1}$ ?

From Schureman the equilibrium argument $\left(V_{o}+u\right)$ is the sum of the values for year, month, day, and hour shown in table III-2.

The Fourier coefficients at station $\mathrm{K}$ at depth $60 \mathrm{~m}$ are taken from this atlas (tables 4-9) and listed in table III-3 along with the phase lag $\phi_{\mathrm{i}}$ computed from eq. III-2.

Substituting into eq. III-1 gives for the tidal currents:

$$
\begin{aligned}
\mathrm{U}_{\text {east }}(\mathrm{cm} / \mathrm{s})= & 22.9 \cos \left(28.984 \mathrm{t}-136^{\circ}\right) \\
+ & 3.3 \cos \left(30.000 \mathrm{t}-328^{\circ}\right)+ \\
& 4.6 \cos \left(28.440 \mathrm{t}-062^{\circ}\right) \\
+ & 2.6 \cos \left(15.041 \mathrm{t}-027^{\circ}\right)+ \\
& 1.2 \cos \left(13.943 \mathrm{t}-281^{\circ}\right) \\
\mathrm{U}_{\text {north }}(\mathrm{cm} / \mathrm{s})= & 31.6 \cos \left(28.984 \mathrm{t}-028^{\circ}\right) \\
+ & 4.7 \cos \left(30.000 \mathrm{t}-222^{\circ}\right)+ \\
& 6.6 \cos \left(28.440 \mathrm{t}-316^{\circ}\right) \\
+ & 2.3 \cos \left(15.041 \mathrm{t}-310^{\circ}\right)+ \\
& 1.2 \cos \left(13.943 \mathrm{t}-204^{\circ}\right)
\end{aligned}
$$

and after similar calculations, the sea-level elevation is:

$$
\begin{aligned}
\mathrm{H}(\mathrm{cm})= & 41.4 \cos \left(28.984 \mathrm{t}-038^{\circ}\right) \\
+ & 8.6 \cos \left(30.000 \mathrm{t}-218^{\circ}\right)+ \\
& 10.3 \cos \left(28.440 \mathrm{t}-334^{\circ}\right) \\
+ & 6.5 \cos \left(15.041 \mathrm{t}-045^{\circ}\right)+ \\
& 5.0 \cos \left(13.943 \mathrm{t}-331^{\circ}\right)
\end{aligned}
$$


Table III-2. Calculation of Greenwich $\left(V_{o}+u\right)$

\begin{tabular}{lrrrrrr}
\hline & $\mathrm{M}_{2}$ & \multicolumn{1}{c}{$\mathrm{S}_{2}$} & \multicolumn{1}{c}{$\mathrm{N}_{2}$} & \multicolumn{1}{c}{$\mathrm{K}_{1}$} & \multicolumn{1}{c}{$\mathrm{O}_{1}$} & Reference \\
\hline 1978 & 201.80 & 0.00 & 290.60 & 10.50 & 191.30 & p. 210, Table 15 \\
February & 324.17 & 0.00 & 279.16 & 30.56 & 293.62 & p. 212, Table 16 \\
1 & 0.00 & 0.00 & 0.00 & 0.00 & 0.00 & p. 213-5, Table 17 \\
0100 & 28.98 & 30.00 & 28.44 & 15.04 & 13.94 & p. 216-7, Table 18 \\
\hline Greenwich & & & & & & \\
$\left(\mathrm{V}_{\text {o }}+\mathrm{u}\right)$ & 194.95 & 30.00 & 238.20 & 56.10 & 138.86 &
\end{tabular}

Table III-3. Fourier coefficients and computation of $\phi_{\mathrm{i}}$

\begin{tabular}{|c|c|c|c|c|c|c|c|c|c|c|}
\hline \multirow[b]{2}{*}{$\begin{array}{l}\text { Amplitude } \\
\mathrm{u}(\mathrm{cm} / \mathrm{s})\end{array}$} & \multicolumn{2}{|c|}{$M_{2}$} & \multicolumn{2}{|c|}{$\mathrm{S}_{2}$} & \multicolumn{2}{|c|}{$\mathrm{N}_{2}$} & \multicolumn{2}{|c|}{$K_{1}$} & \multicolumn{2}{|c|}{$\mathrm{O}_{1}$} \\
\hline & $\begin{array}{l}\text { east } \\
22.1\end{array}$ & $\begin{array}{r}\text { north } \\
30.4\end{array}$ & $\begin{array}{r}\text { east } \\
3.3\end{array}$ & $\begin{array}{r}\text { north } \\
4.7\end{array}$ & $\begin{array}{r}\text { east } \\
4.4\end{array}$ & $\begin{array}{r}\text { north } \\
6.4\end{array}$ & $\begin{array}{r}\text { east } \\
3.0\end{array}$ & $\begin{array}{r}\text { north } \\
2.6\end{array}$ & $\begin{array}{r}\text { east } \\
1.5\end{array}$ & $\begin{array}{r}\text { north } \\
1.5\end{array}$ \\
\hline \multicolumn{11}{|l|}{ Phase } \\
\hline Greenwich (G) & $116^{\circ}$ & $8^{\circ}$ & $148^{\circ}$ & $42^{\circ}$ & $82^{\circ}$ & $336^{\circ}$ & $158^{\circ}$ & $81^{\circ}$ & $130^{\circ}$ & $53^{\circ}$ \\
\hline - Greenwich $\left(V_{0}+u\right)$ & $-195^{\circ}$ & $-195^{\circ}$ & $-30^{\circ}$ & $-30^{\circ}$ & $-238^{\circ}$ & $-238^{\circ}$ & $-56^{\circ}$ & $-56^{\circ}$ & $-139^{\circ}$ & $-139^{\circ}$ \\
\hline$-\omega_{i} S / 15$ & $-145^{\circ}$ & $-145^{\circ}$ & $-150^{\circ}$ & $-150^{\circ}$ & $-142^{\circ}$ & $-142^{\circ}$ & $-75^{\circ}$ & $-75^{\circ}$ & $-70^{\circ}$ & $-70^{\circ}$ \\
\hline Phase lag $\phi_{i}$ & $136^{\circ}$ & $28^{\circ}$ & $328^{\circ}$ & $222^{\circ}$ & $62^{\circ}$ & $316^{\circ}$ & $27^{\circ}$ & $310^{\circ}$ & $281^{\circ}$ & $204^{\circ}$ \\
\hline
\end{tabular}



PLATES 3-12 AND 14-21

(Plates 1, 2, and 13 are in pocket) 



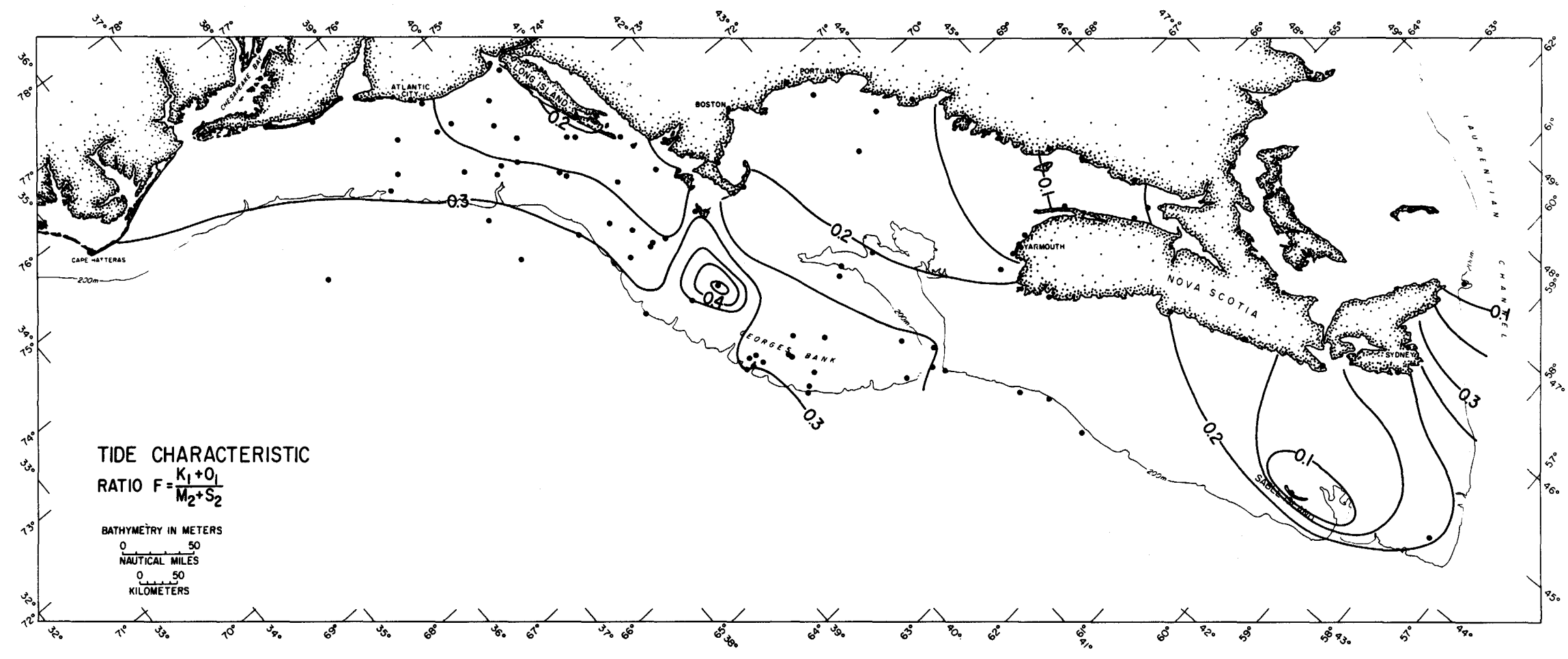

Plate 3. F values for study region. 


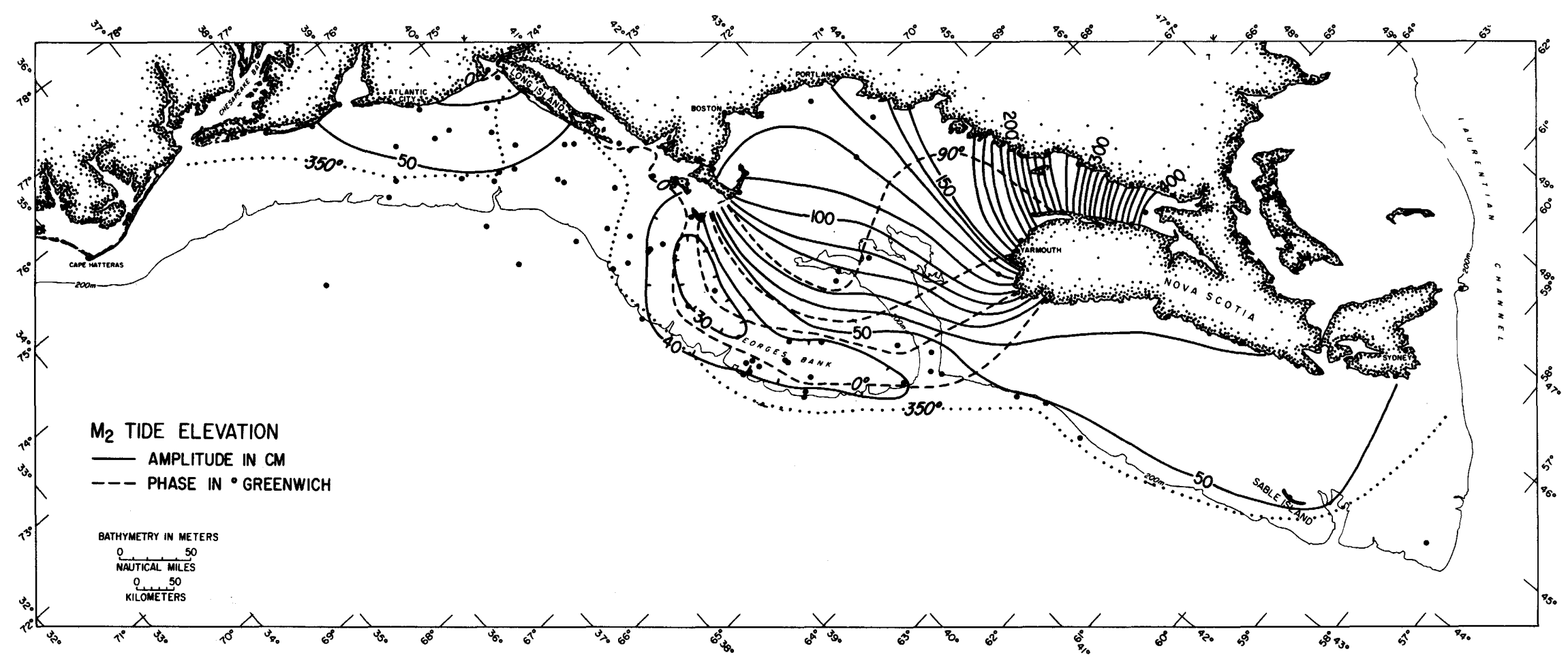

Plate 4. Coamplitude and cophase lines for the $M_{2}$ (12.42 hours) semidiurnal tide. The tide is in co-oscillation across the Middle Atlantic and Scotian Shelves and near resonance in the Gulf of Maine. The $350^{\circ}$ phase line (shown dotted to emphasize a change in phase contour interval) has been added to indicate the simultaneous arrival of the tide along the entire shelf break and so that the phase can be compared to existing North Atlantic cotidal charts (see Defant, 1958; Beaumont and Boutilier, 1978). Note the amplitude minimum over Nantucket Shoals and the southern flank of Georges Bank. 


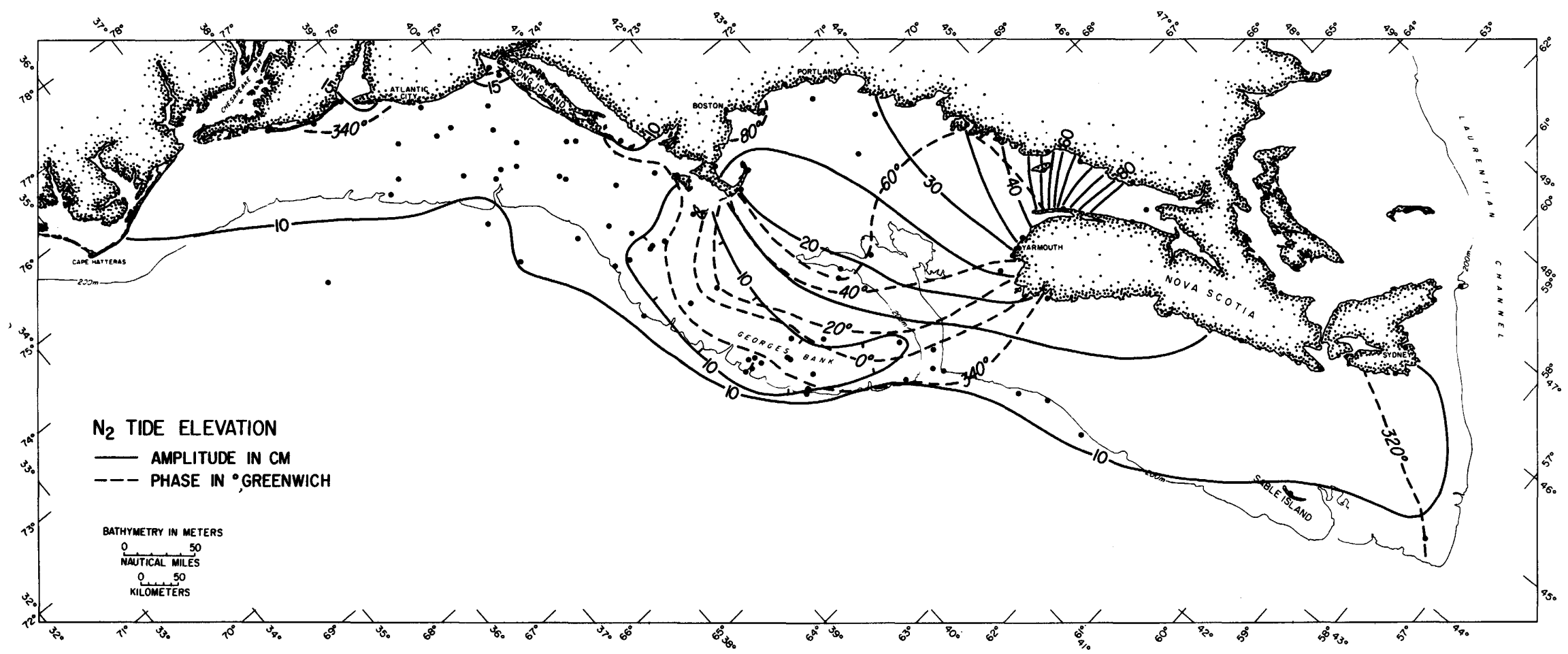

Plate 5. Coamplitude and cophase lines for the $N_{2}$ (12.66 hours) semidiurnal tide. Cophase lines are similar to the $M_{2}$ tide and the amplitudes are approximately 25 percent of the amplitude of the $\mathrm{M}_{2}$. 


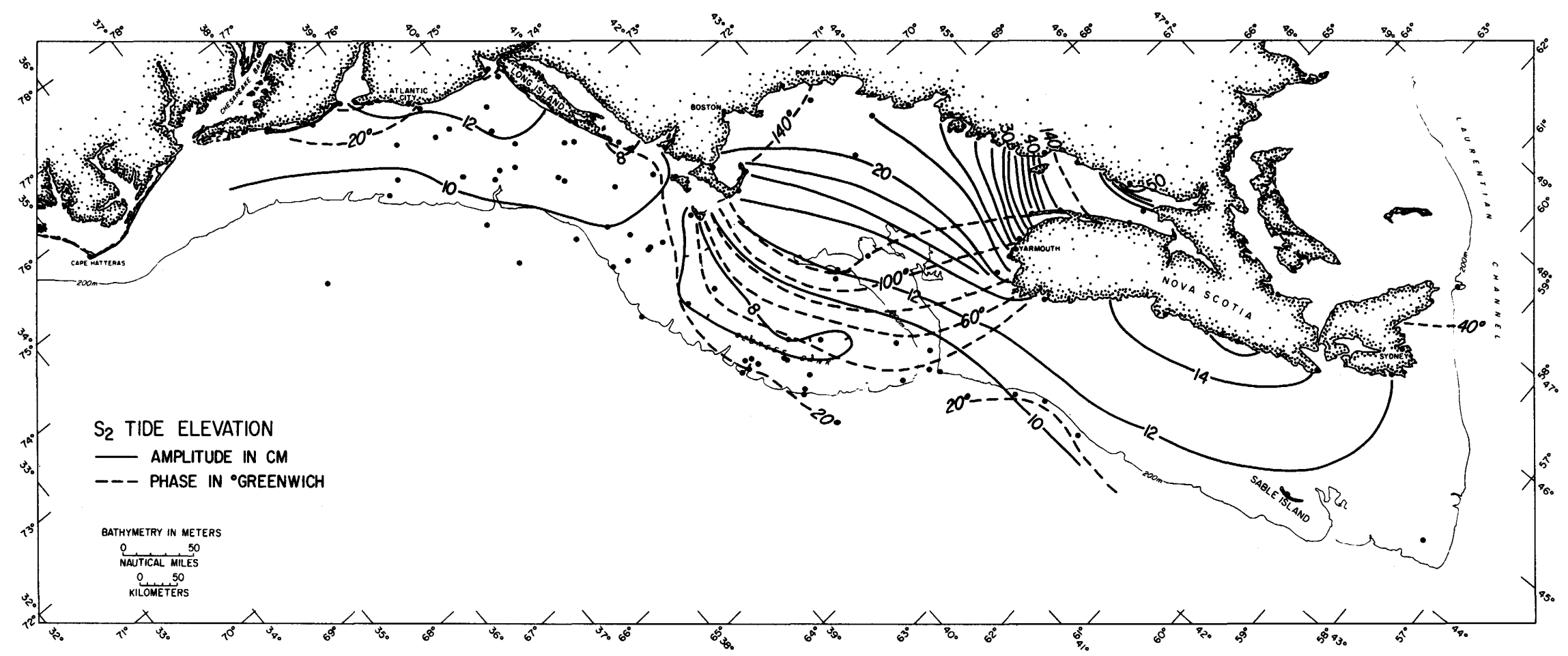

Plate 6. Coamplitude and cophase lines for the $S_{2}$ (12.00 hours) solar semidiurnal tide. 


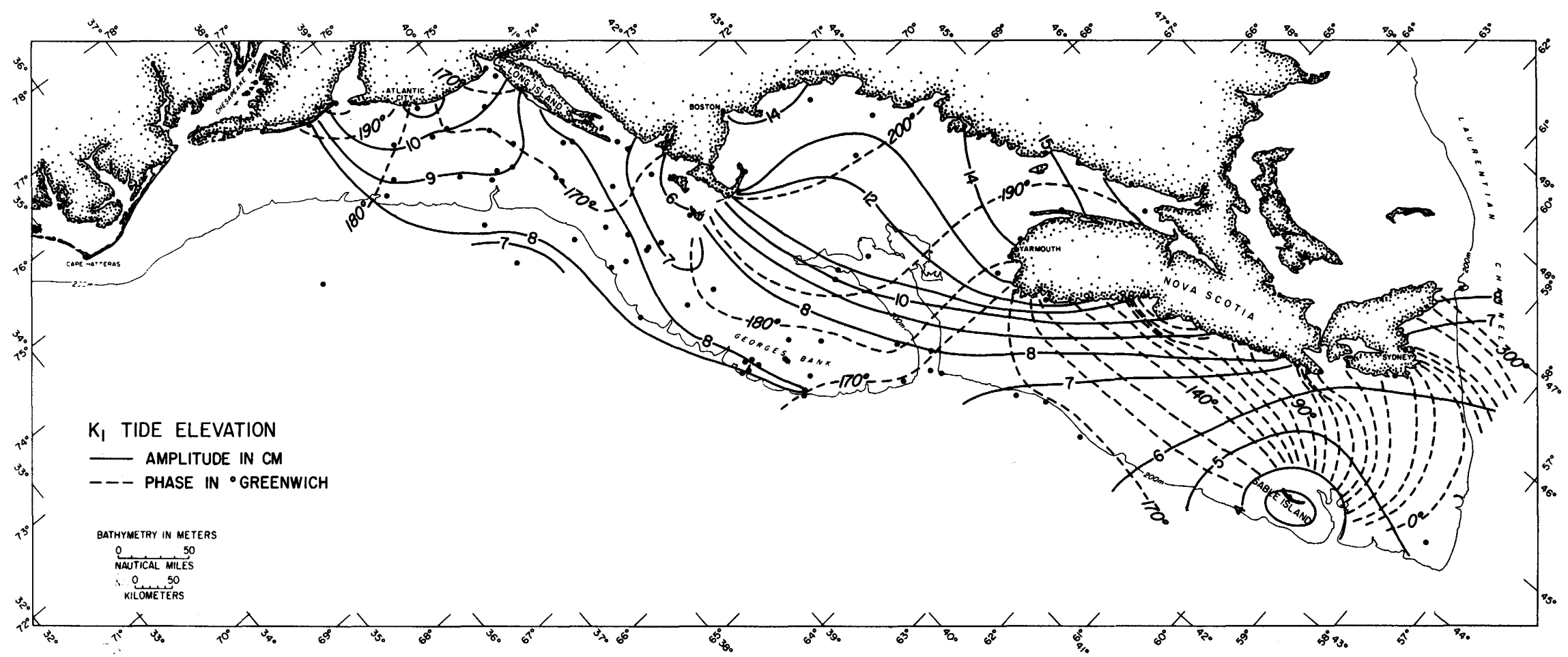

Plate 7. Coamplitude and cophase lines for the $K_{1}$ (23.93 hours) diurnal tide. There are three amphidromes, one near Sable Island, one near Cape Cod, and one near Atlantic City, N. J. The open circles indicate the locations of additional stations (not included in table 4) used to contour the amphidromic system near Sable Island (Canadian Hydrographic Service, 1969a). 


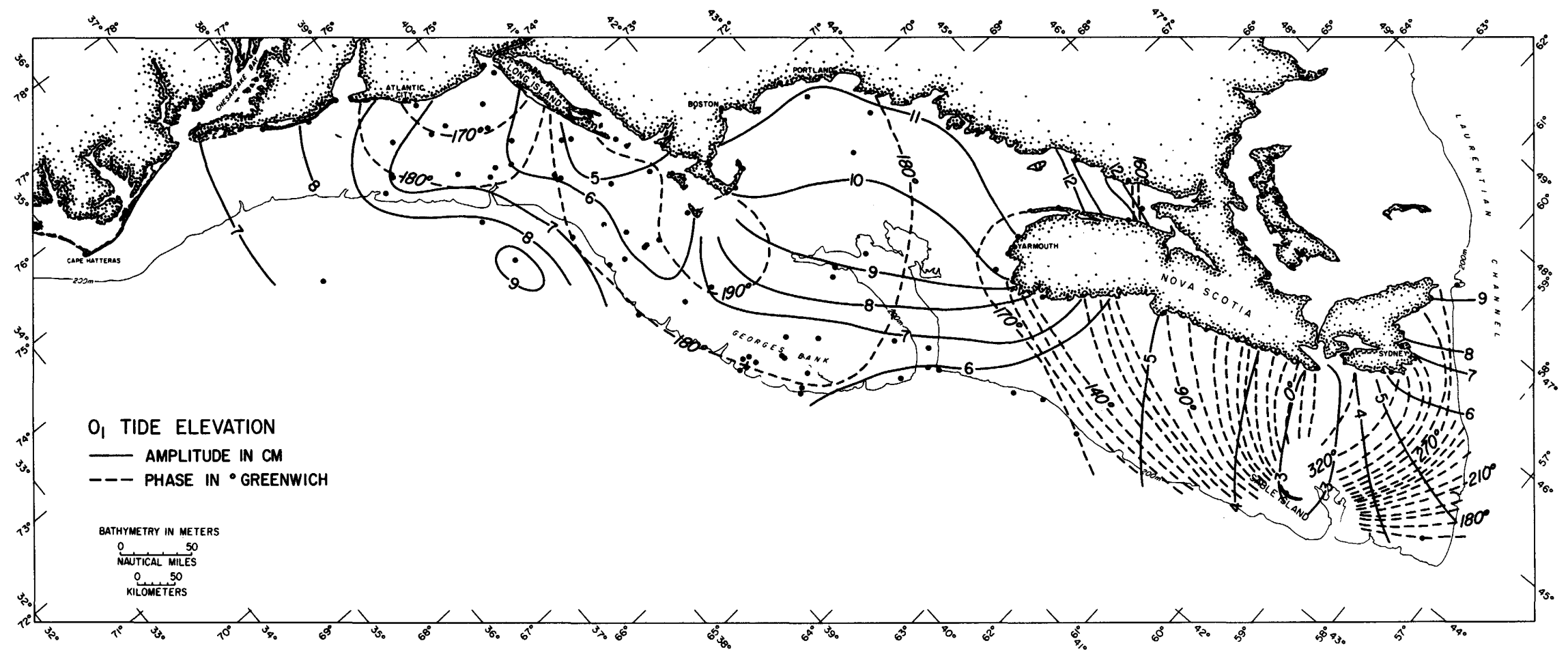

Plate 8. Coamplitude and cophase lines for the $\mathrm{O}_{1}(25.82$ hours) diurnal tide. Notice the amphidromic point near Sable Island and the unusual increase in amplitude across the shelf just east of Long Island. The open circles represent the location of additional stations (not in table 4) used to contour the amphidromic system near Sable Island (Canadian Hydrographic Service, 1969a). 


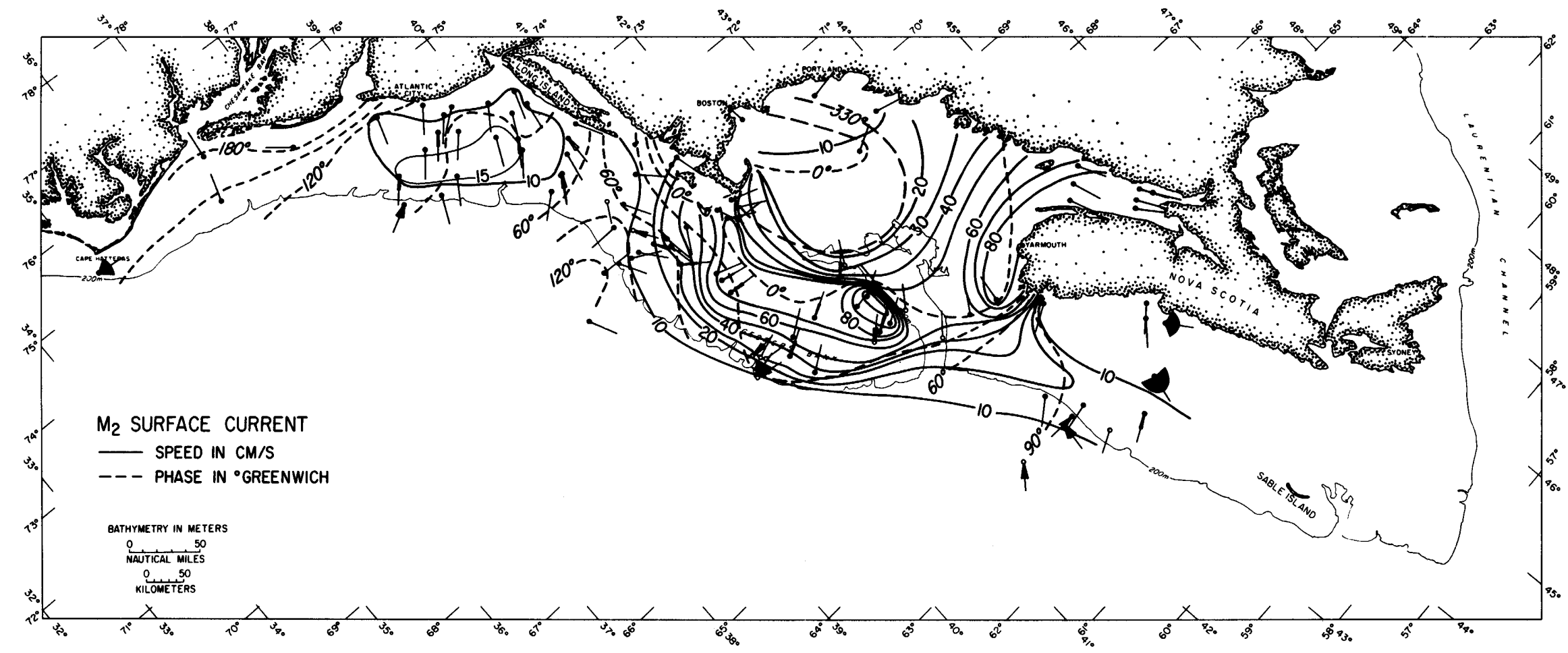

Plate 9. Magnitude of the major axis (UMAJOR) and phase of the $M_{2}$ surface current. The observations used to draw this map of surface currents were obtained at depths less than $35 \mathrm{~m}$ from the sea surface (plate 2) and are indicated by solid circles. The lines indicate the ellipse orientation. The standard deviation of the ellipse orientation is shown by shaded sectors at stations where the standard deviation was computed. The phase of maximum current is relative to the indicated orientation. Note that the ellipse orientation and phase may be adjusted by $180^{\circ}$. The ellipse orientation was selected so that the phase of the current varied smoothly. To expand the spatial coverage, observations at depths greater than $35 \mathrm{~m}$ were used in some locations (indicated by open circles). At these locations, the tide was assumed to be barotropic. 


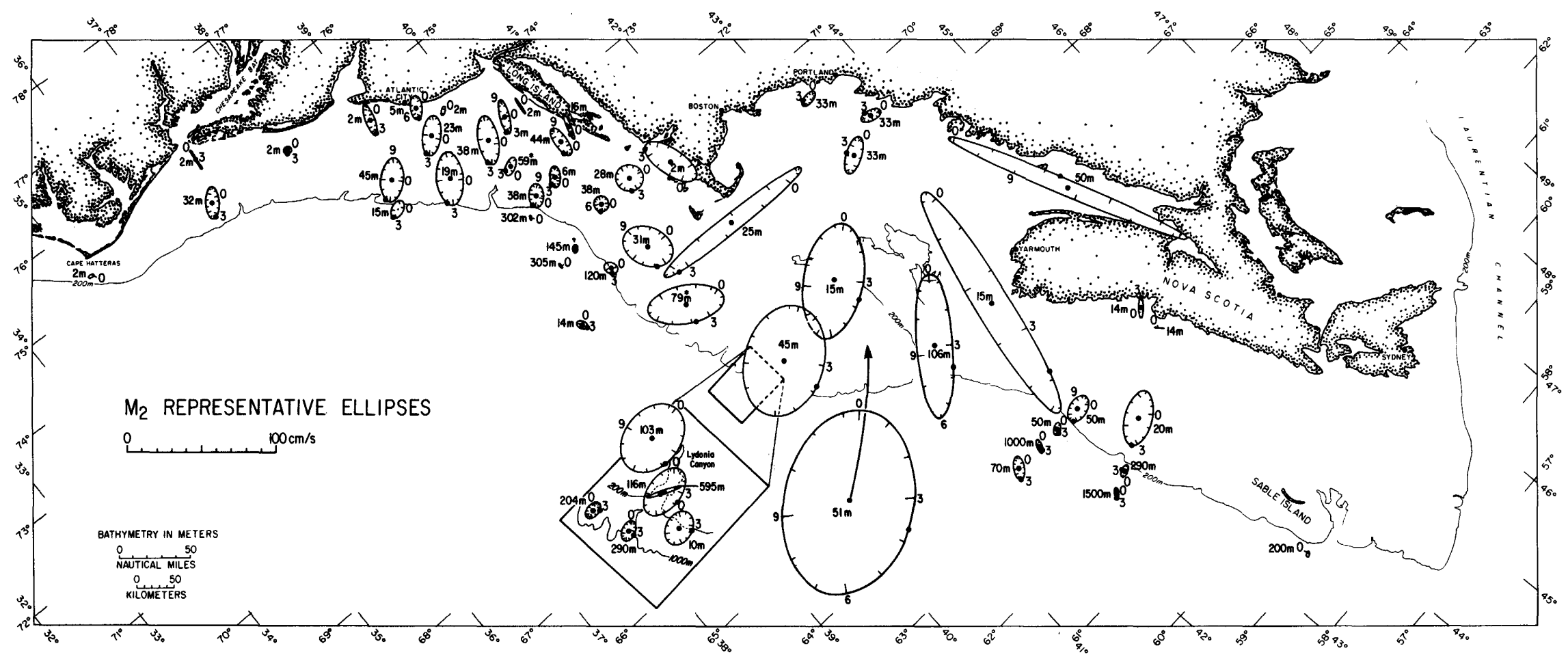

Plate 10. Representative $M_{2}$ tidal ellipses. Ellipses were selected to be representative and located at approximately mid-water depth. Numbers inside or near the ellipses are the instrument depths in meters below the sea surface. Tick marks along the edge of the ellipse indicate the Greenwich hour. The time of Boston high water is shown by a solid circle between 3 - and 4 hour Greenwich. Asterisks are stations 1 mab. 


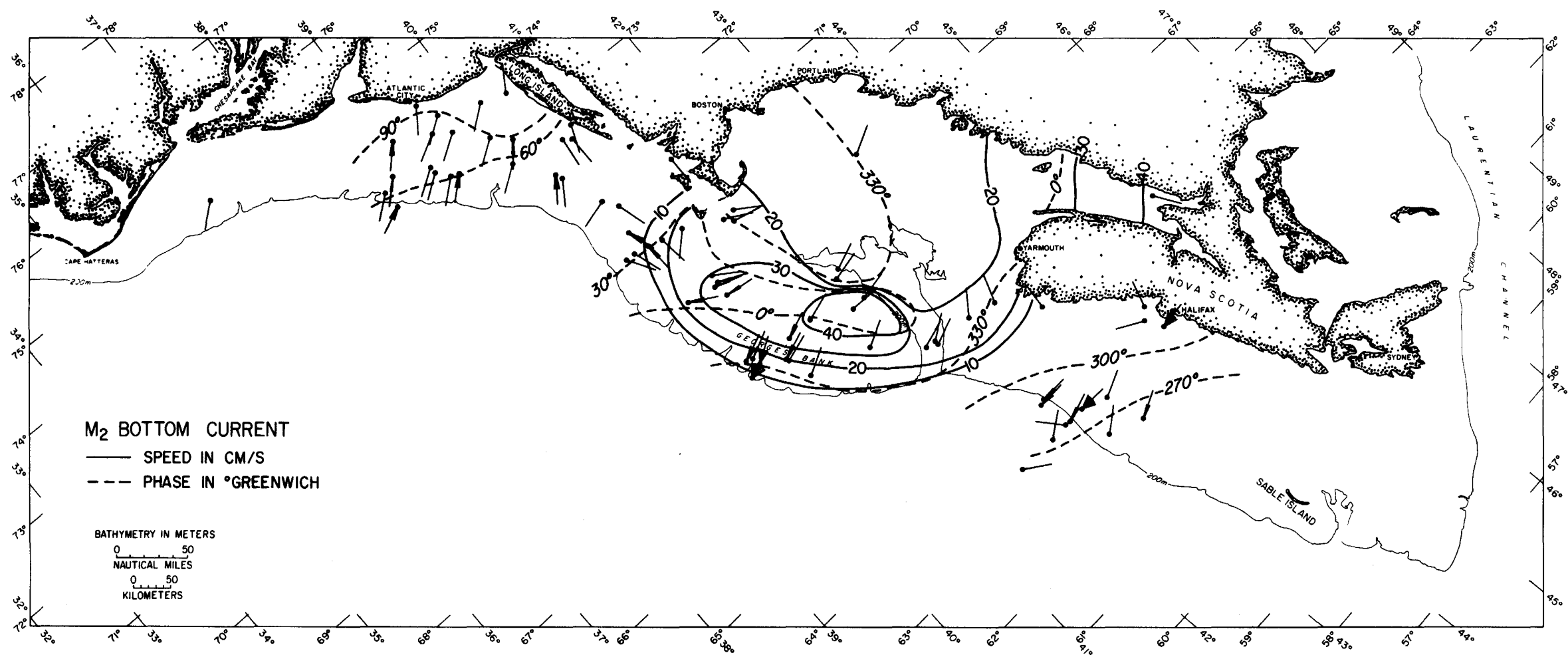

Plate 11. $M_{2}$ currents at 1 meter above bottom. Current measurements made at 14 stations and estimates (based on an empirical curve and near-bottom measurements) at 64 additional stations were used to contour phase and maximum speed. See text for discussion of the empirical curve used and appendix II for measured and computed values of the tidal current 1 mab. All phases are relative to the orientations shown. The shaded sectors indicate the estimated uncertainty in the ellipse orientation. 


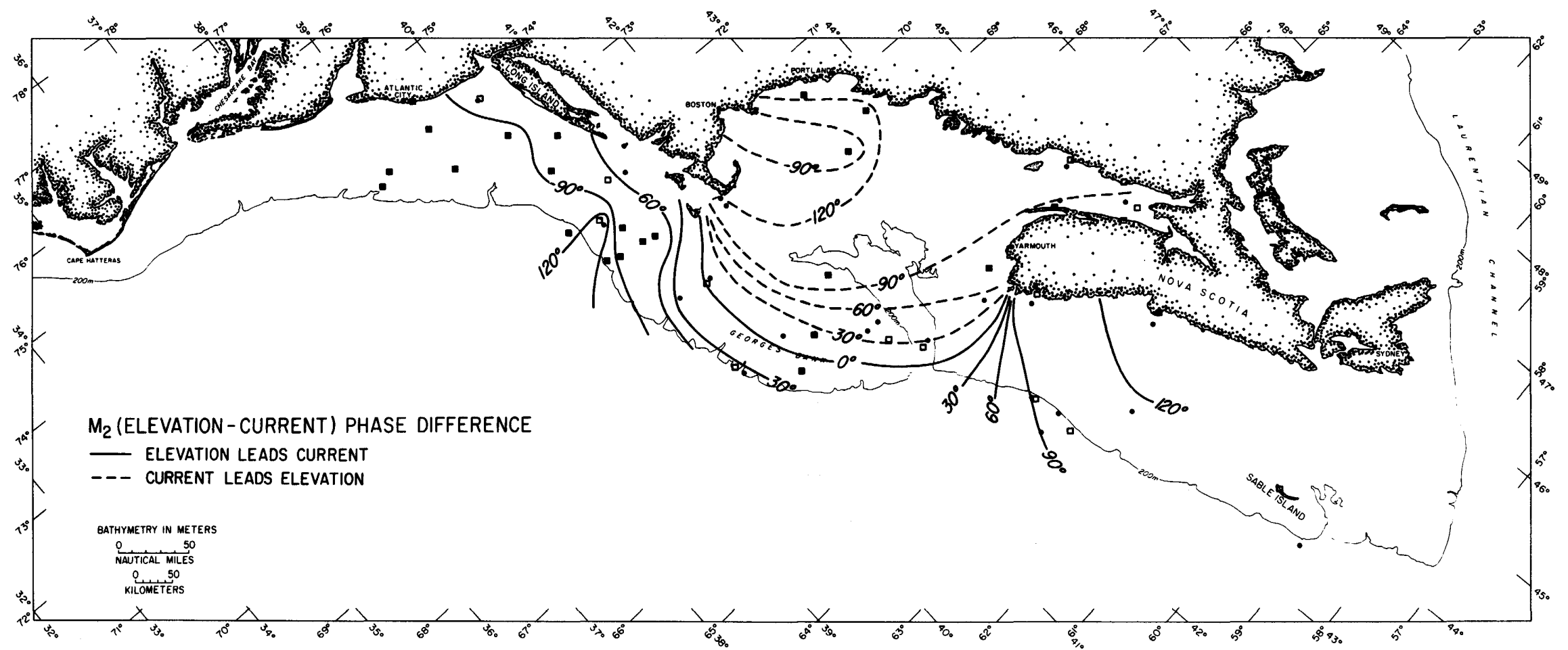

Plate 12. Phase difference (elevation minus current) for the $M_{2}$ tide. The open squares are the locations of tidal elevation data and the solid circles are the location of current data used to draw the lines of equal phase difference. Solid squares are locations for which both tidal elevation and current data were available. The average standard deviation of the phase difference was $<10^{\circ}$. 


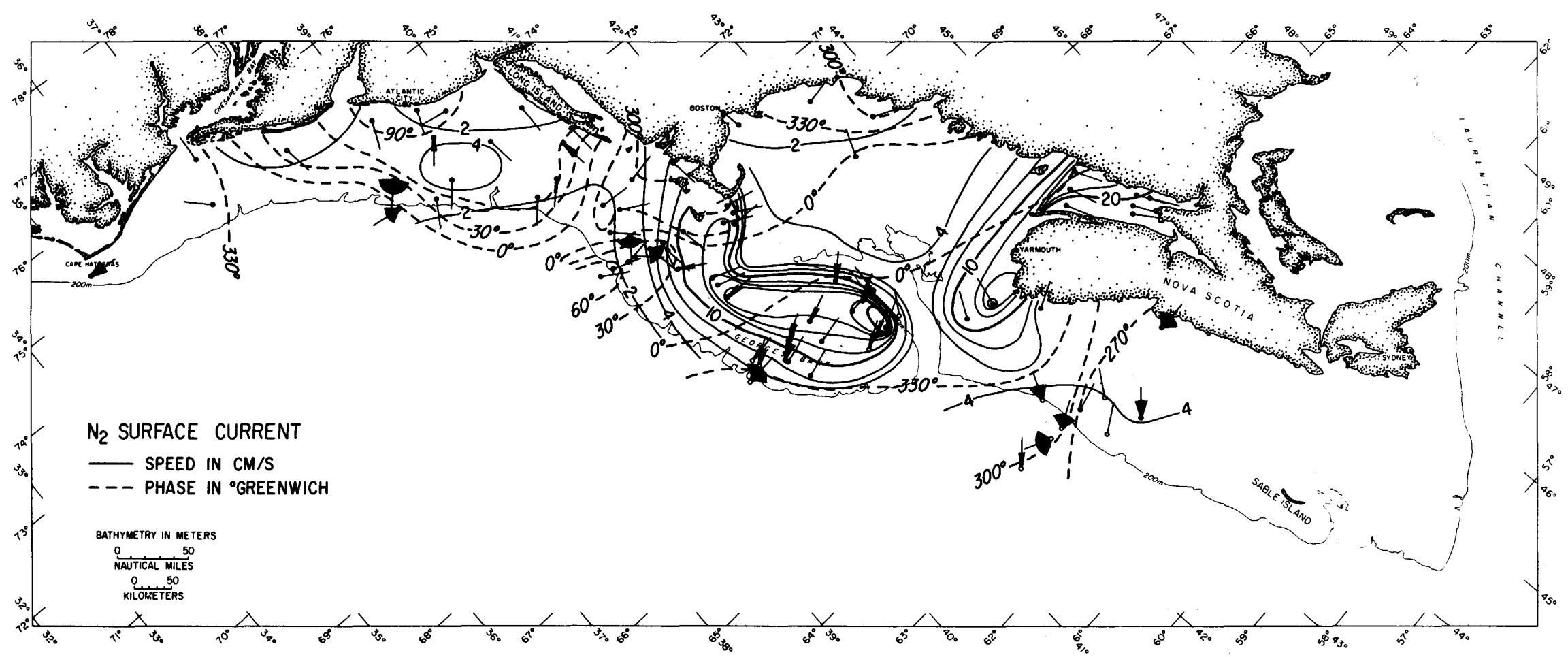

Plate 14. Magnitude of the major axis (UMAJOR) and phase of the $N_{2}$ surface current. See plate 9 for explanation of symbols. 


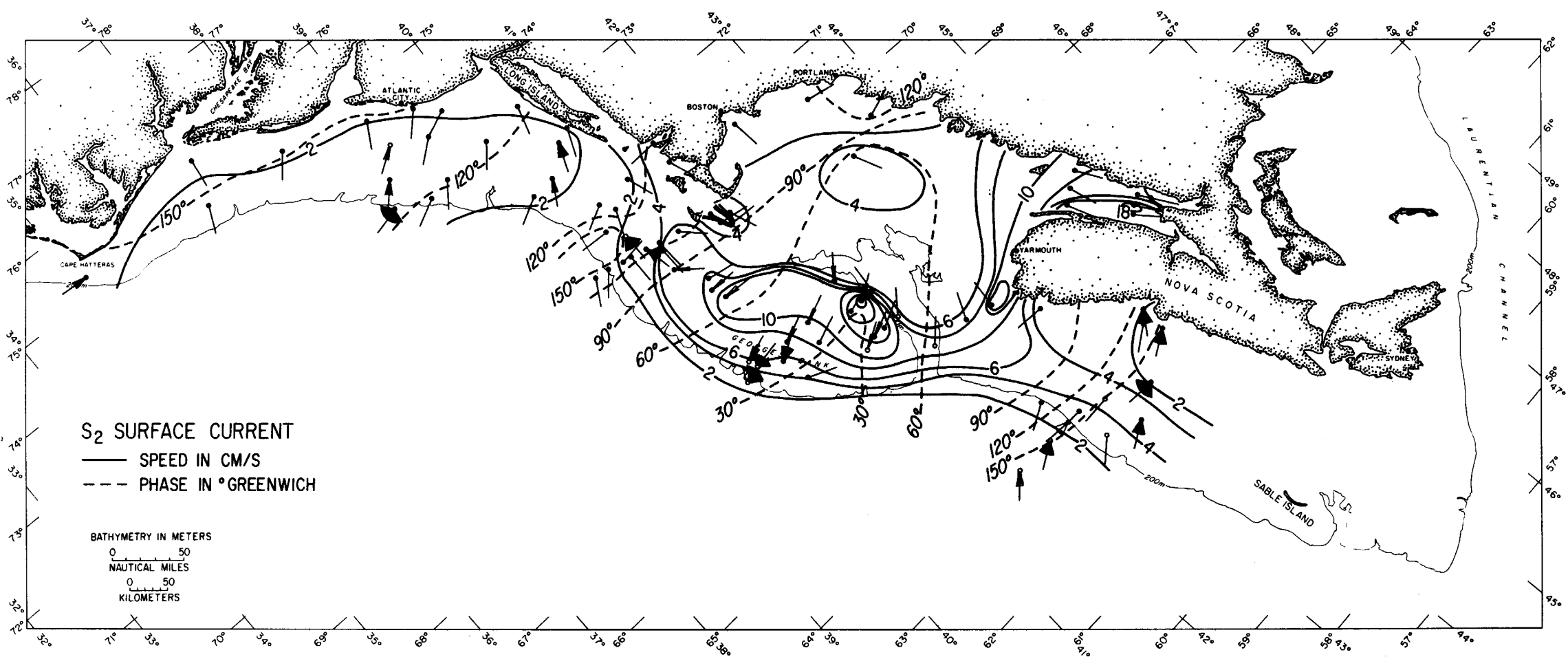

Plate 15. Magnitude of the major axis (UMAJOR) and phase of the $S_{2}$ surface current. See plate 9 for explanation of symbols. 


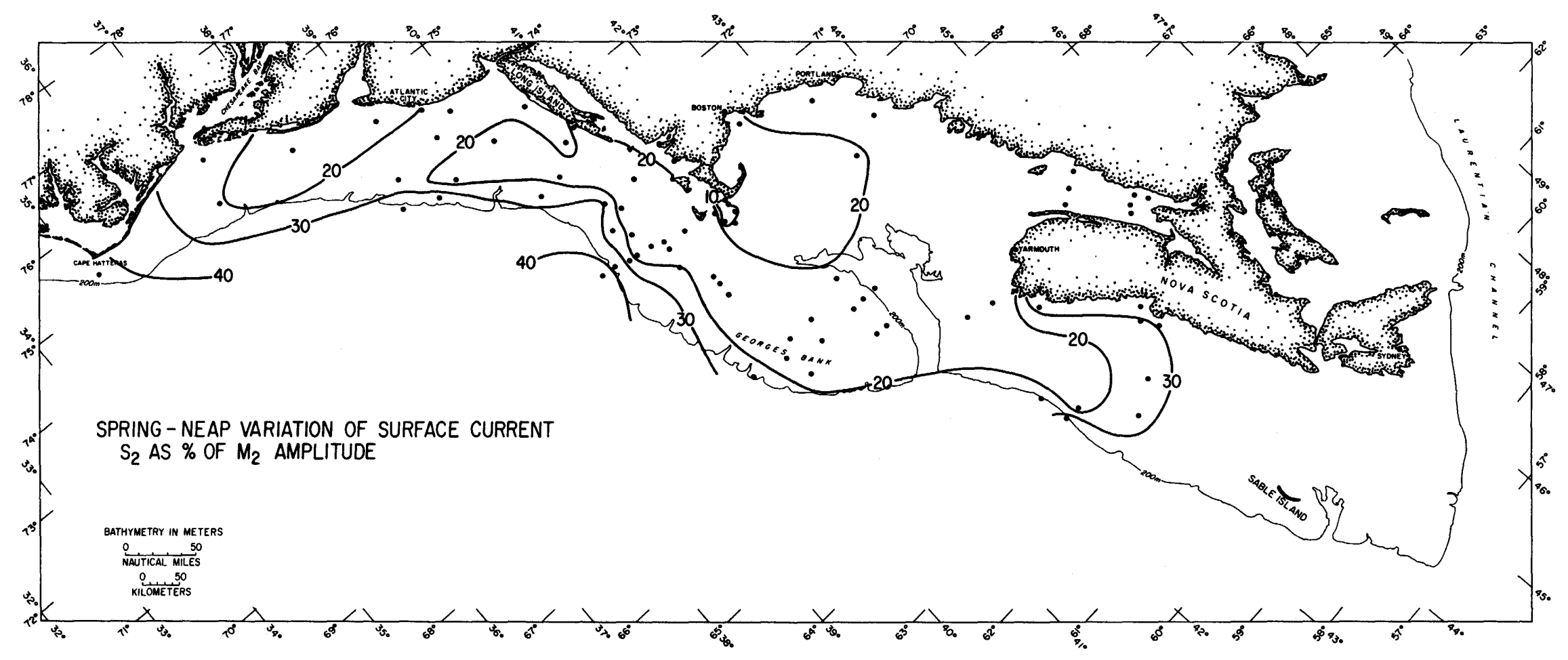

Plate 16. Spring-neap variation of the amplitude of the semidiurnal tidal current caused by the $S_{2}$ solar tide expressea as a percent of the $M_{2}$ tidal current. 


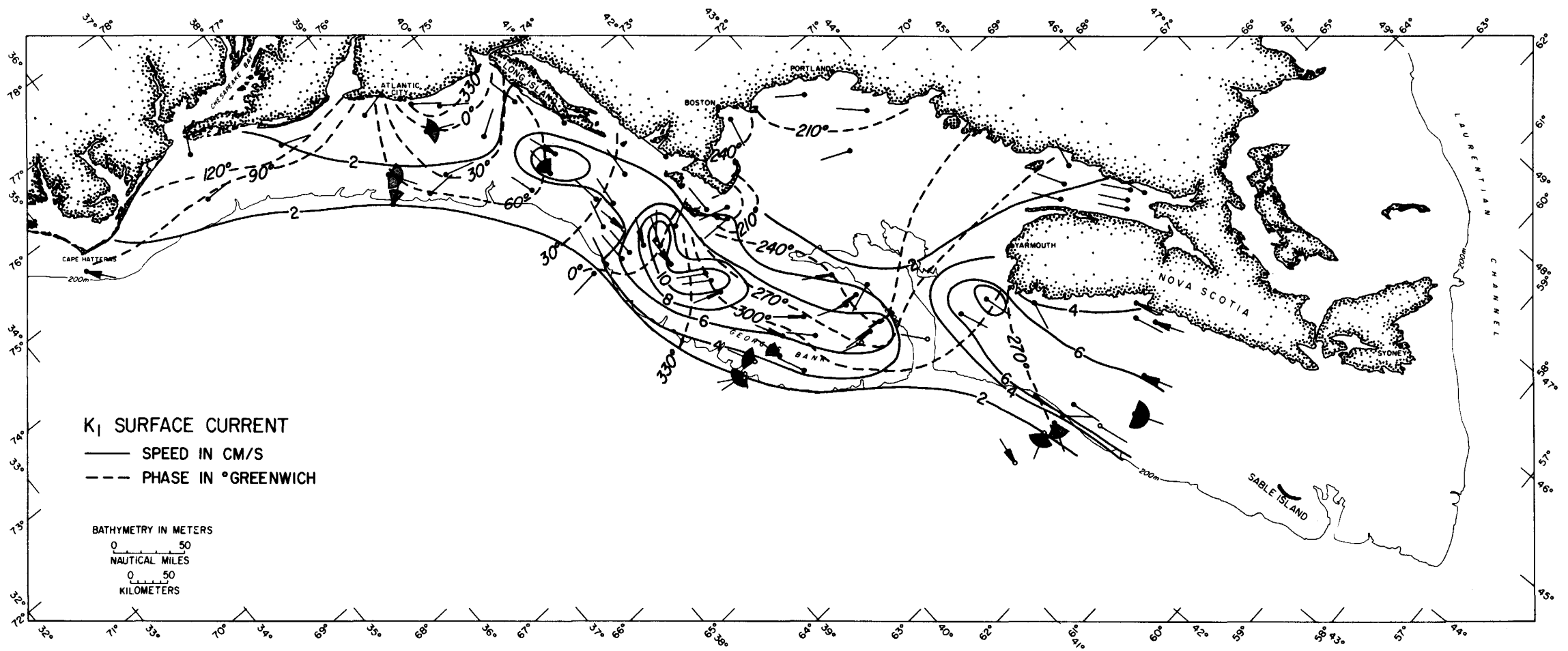

Plate 17. Magnitude of the major axis (UMAJOR) and phase of the $K_{1}$ surface current. See plate 9 for explanation of symbols. 


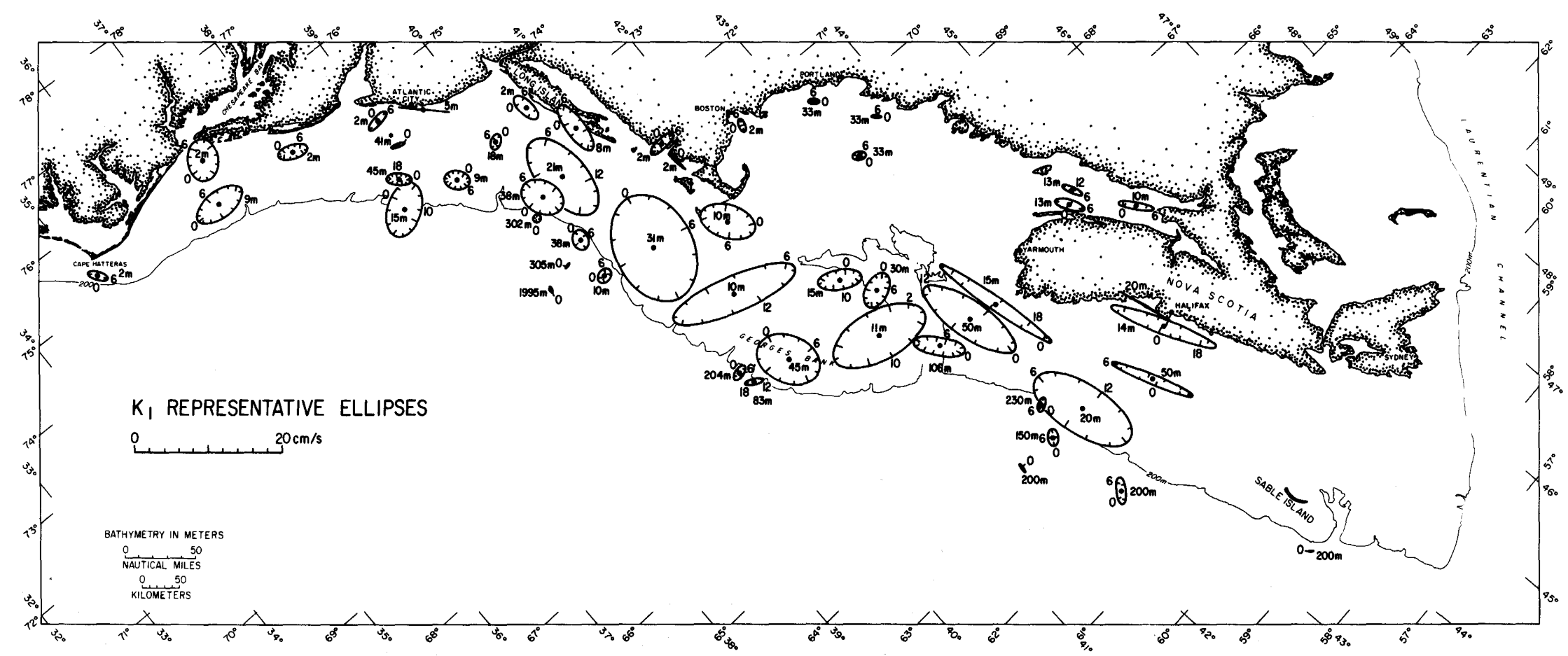

Plate 18. Representative $K_{1}$ tidal ellipses. Note that these ellipses are not drawn at the same scale as the $M_{2}$ ellipses in plate 10. Ellipses were selected to be representative and located at approximately midwater depth. Numbers inside or near the ellipses are the instrument depths in meters. Tick marks along the edge of the ellipse indicate the Greenwich hour. 


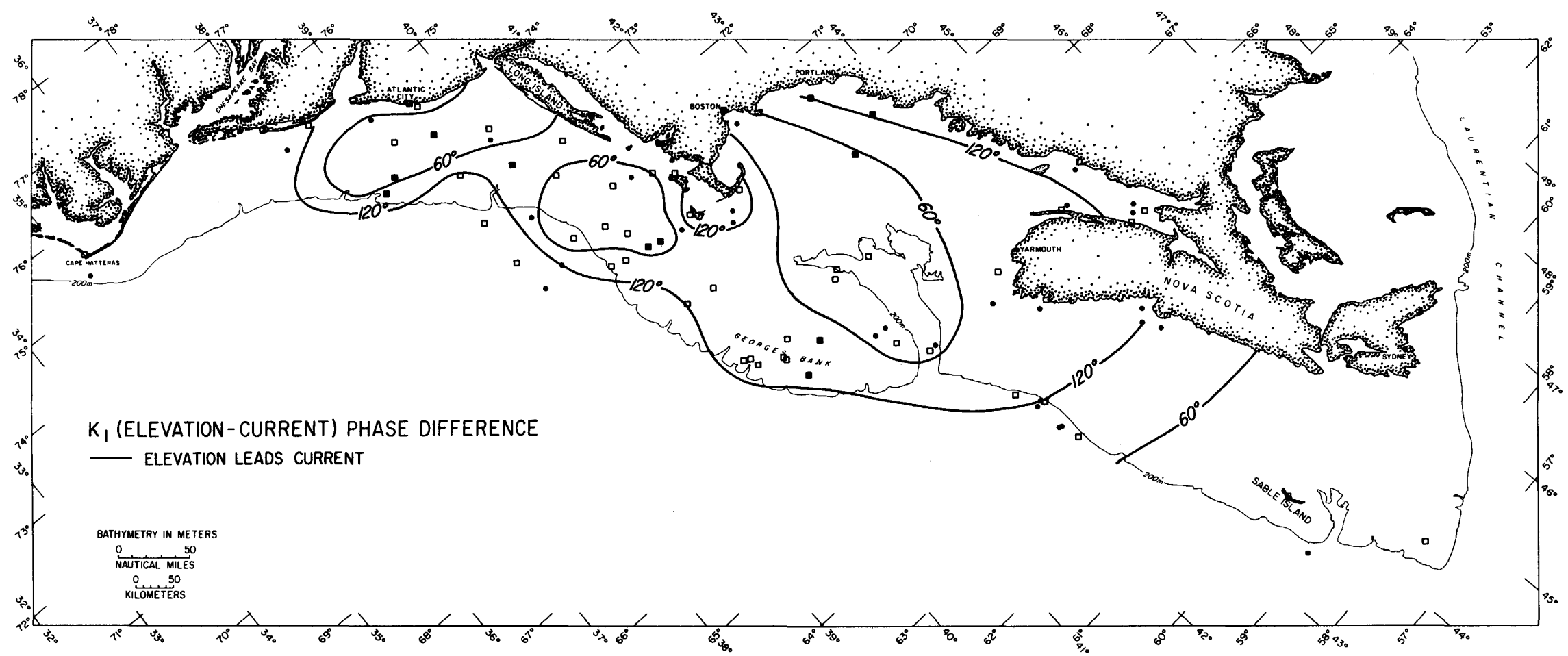

Plate 19. Phase difference (elevation minus current) for the $K_{1}$ tide. The open squares are the locations of tidal elevation data and the solid circles are the locations of current data used to draw the lines of equal phase difference. Solid squares are locations with both tidal elevation and current data. The uncertainty (average standard deviation) in the phase difference is approximately $\pm 33^{\circ}$. 


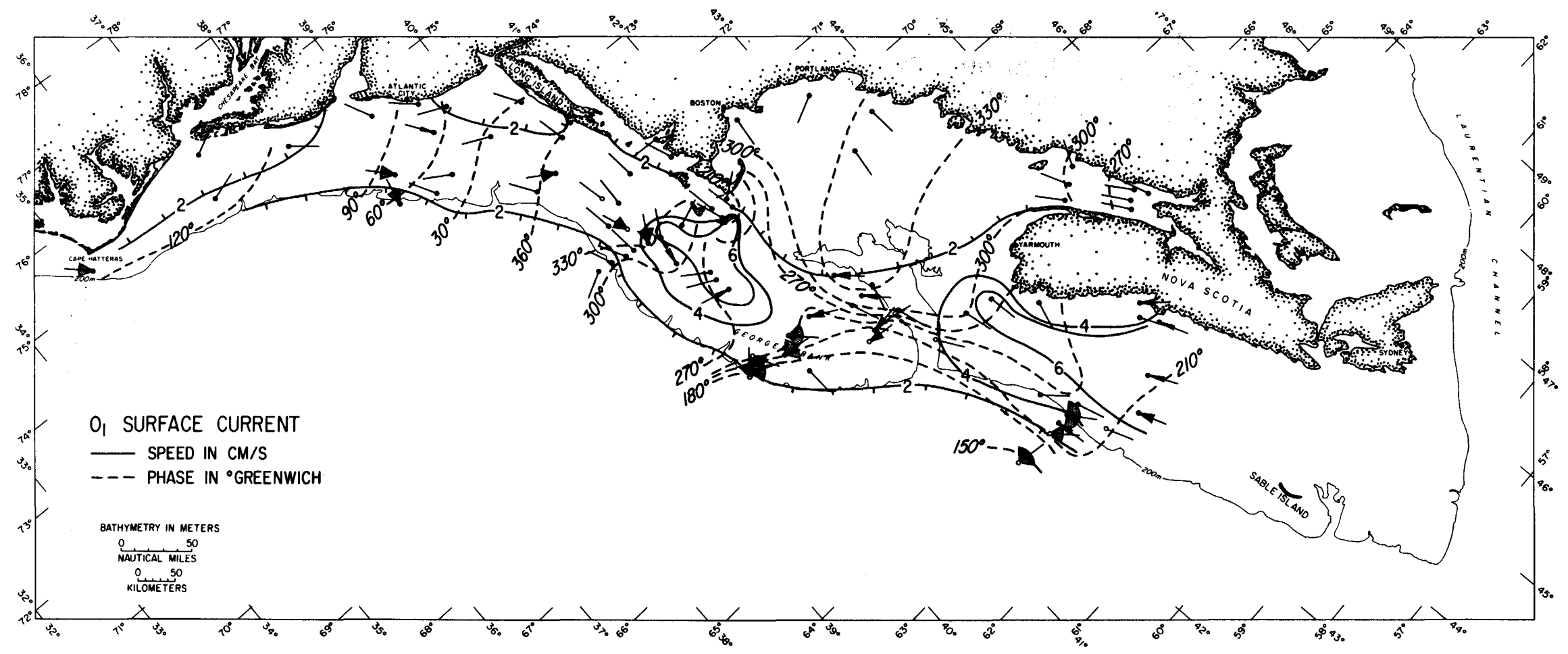

Plate 20. Magnitude of the major axis (UMAJOR) and phase of the $O_{1}$ surface current. See plate 9 for explanation of symbols. 


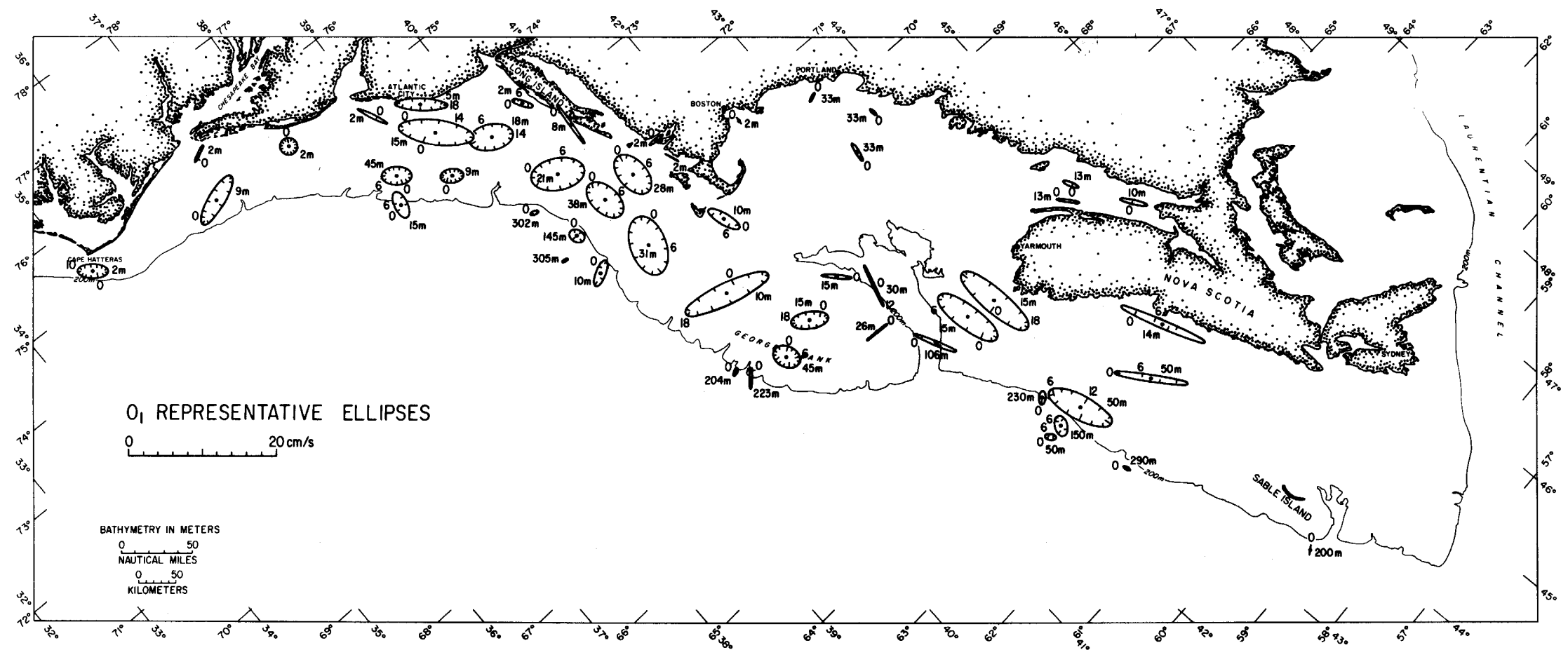

Plate 21. Representative $O_{1}$ tidal ellipses. These ellipses are drawn at the same scale as the $K_{1}$ ellipses (plate 18) but not at the same scale as the $M_{2}$ ellipses (plate 10). See plate 18 for explanation of symbols. 

